OAK RIDGE NATIONAL LABORATORY

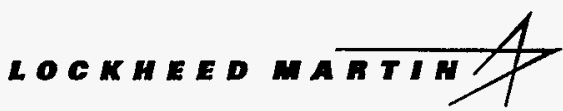

WANAGED AND OPERATED BY LOCKHEED MARTIN ENERGY RESEARCH CORPORATION FOR THE UAMTED STATES DEPARTMENT OF ENERGY

\section{Characterization of the \\ Old Hydrofracture Facility \\ (OHF) \\ Waste Tanks \\ Located at ORNL}

J. M. Keller

J. M. Giaquinto

A. M. Meeks

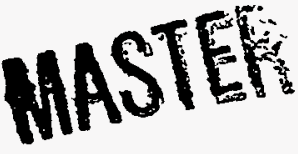

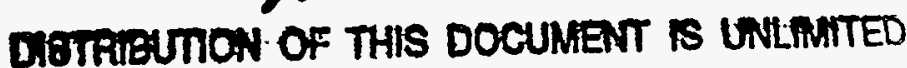


This report has been reproduced directly from the best available copy.

Available to DOE and DOE contractors from the Office of Scientific and Technical Information, P. O. Box 62, Oak Ridge, TN 37831; prices available from (423) 576-8401. FTS 626-8401.

Available to the public from the National Technical Information Service, U.S. Department of Commerce, 5285 Port Royal Road, Springfield. VA 22161.

This report was prepared as an account of work sponsored by an agency of the United States Government. Neither the United States Government nor any agency thereof, nor any of their employees, makes any warranty, express or implied, or assumes any legal liability or responsibility for the accuracy. completeness, or usefulness of any information, apparatus, product, or process disclosed, or represents that its use would not infringe privately owned rights. Reference herein to any specific commercial product, process, or service by trade name. trademark, manufacturer, or otherwise, does not necessarily constitute or imply its endorsement, recommendation, or favoring by the United States Government or any agency thereof. The views and opinions of authors expressed herein do not necessarily state or reflect those of the United States Government of any agency thereof. 


\section{DISCLAIMER}

Portions of this document may be illegible electronic image products. Images are produced from the best available original document. 
Chemical and Analytical Sciences Division

\section{Characterization of the Old Hydrofracture Facility (OHF) Waste Tanks Located at ORNL}

J. M. Keller

J. M. Giaquinto

A. M. Meeks

April 1997

Prepared by the

OAK RIDGE NATIONAL LABORATORY

Oak Ridge, Tennessee 37831

managed by

LOCKHEED MARTIN ENERGY RESEARCH CORP.

for the

U.S. DEPARTMENT OF ENERGY

under contract

DE-AC05-96OR22464 


\section{TABLE OF CONTENTS}

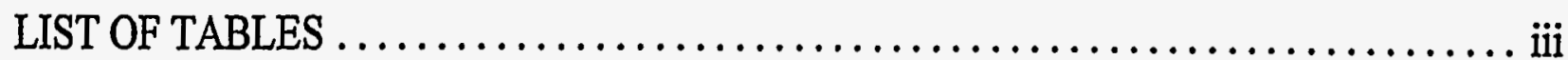

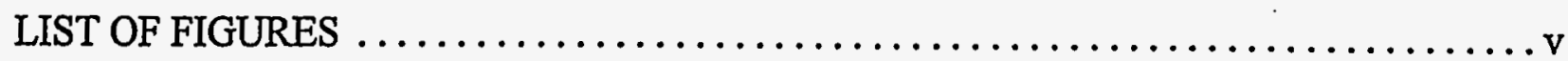

ACKNOWLEDGMENTS $\ldots \ldots \ldots \ldots \ldots \ldots \ldots \ldots \ldots \ldots \ldots \ldots \ldots \ldots \ldots \ldots$ vii

ABBREVIATIONS AND ACRONYMS $\ldots \ldots \ldots \ldots \ldots \ldots \ldots \ldots \ldots \ldots \ldots \ldots \ldots \ldots \ldots$ ix

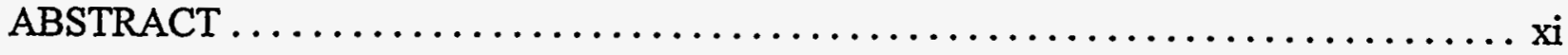

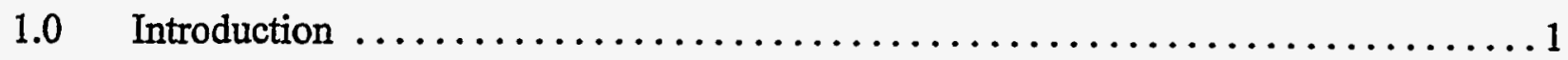

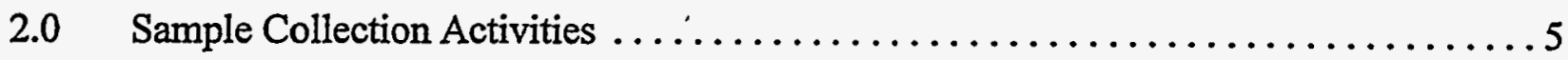

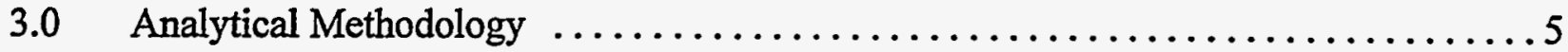

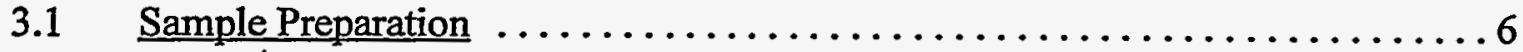

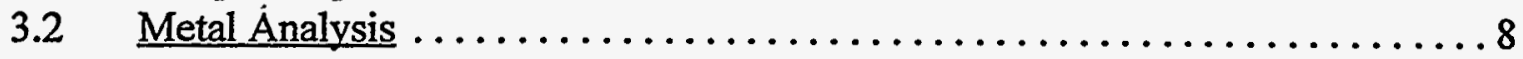

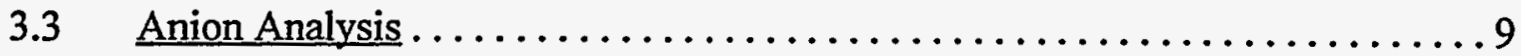

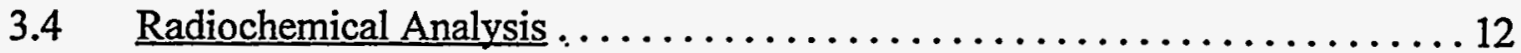

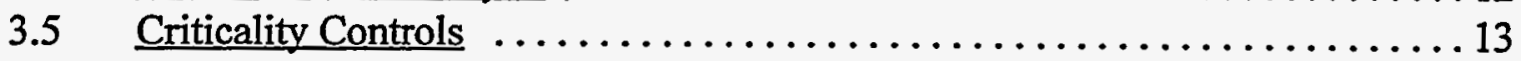

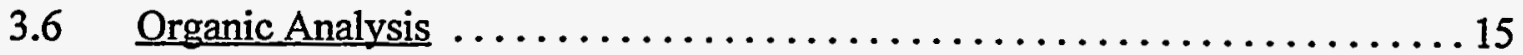

3.6.1 Non-halogenated Volatile Organic Analysis (NHVOA) . . . . . . . 15

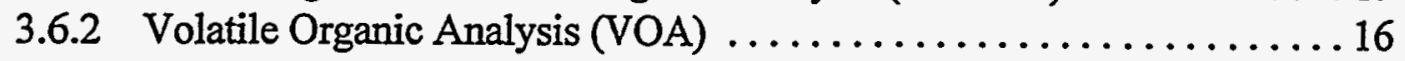

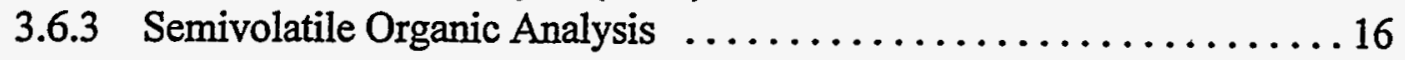

3.6.4 Polychlorinated Biphenyls .......................... 16

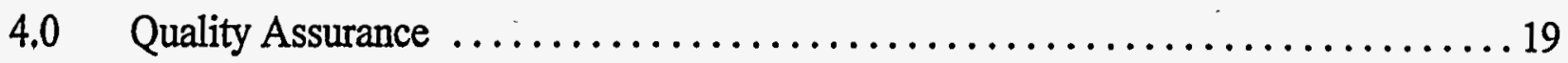

5.0 Summary of Inorganic and Radiochemical Analytical Results ...............21

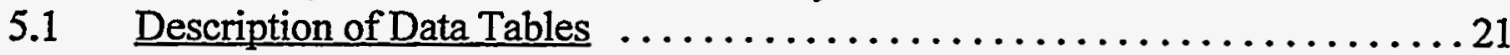

5.2 Discussion of OHF Supernatant Characteristics $\ldots \ldots \ldots \ldots \ldots \ldots \ldots \ldots 6 . \ldots 6$

5.3 Discussion of OHF Sludge Characteristics $\ldots \ldots \ldots \ldots \ldots \ldots \ldots \ldots \ldots 70$

5.4 Inorganic RCRA Characteristics for the OHF System $\ldots \ldots \ldots \ldots \ldots \ldots \ldots 77$

5.5 TRU Classifications for OHF Waste System $\ldots \ldots \ldots \ldots \ldots \ldots \ldots \ldots 78$

5.6 Distribution of Fissile Material in OHF Waste System $\ldots \ldots \ldots \ldots \ldots \ldots 79$

5.7 Discussion of the Total Anion Content in the Sludge $\ldots \ldots \ldots \ldots \ldots \ldots . \ldots 85$

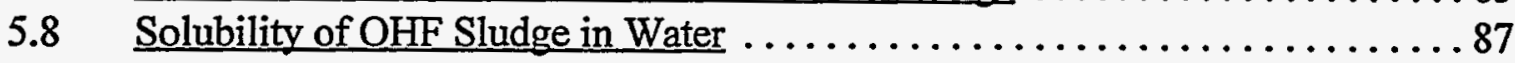

5.9 Estimates for Compliance with WIPP WAC, Rev. 5 for OHF Sludge . . . . . 89

5.9.1 Estimates for ${ }^{239} \mathrm{Pu}$ Fissile Gram Equivalent in OHF Sludge $\ldots . \ldots . .89$

5.9.2 Estimates for ${ }^{239} \mathrm{Pu}$ Equivalent Activity in OHF Sludge .......... 90

5.9.3 Estimates of thermal power from decay heat in OHF sludge .......91 


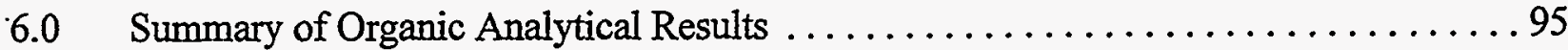

6.1 Discussion of Organic Analysis ........................ 105

6.2 Regulatory Concerns due to Organic Compounds in OHF Waste ......... 106

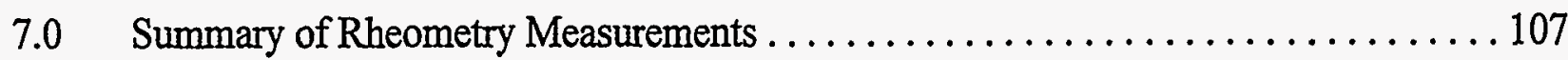

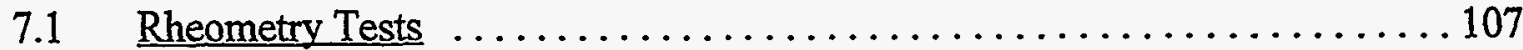

7.2 Shear Strength Measurements ................................ 109

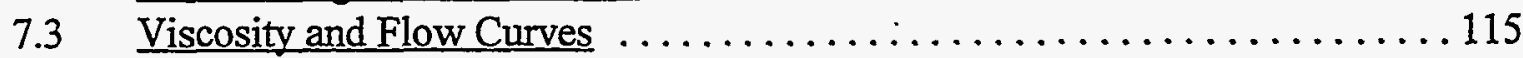

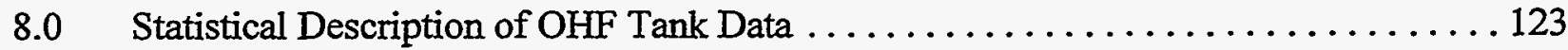

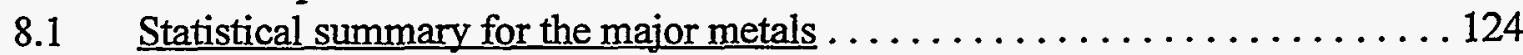

8.2 Statistical summary for the major radionuclides $\ldots \ldots \ldots \ldots \ldots \ldots \ldots 139$

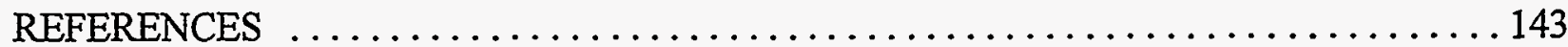

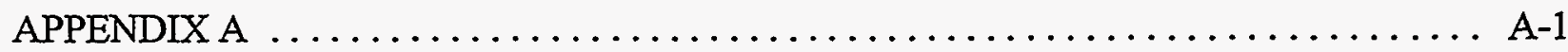

Table A1 Instrument Detection Limits (IDL) for Analytical Measurements ..... A-1

Table A2 QC Acceptance Criteria for Radioactive Liquid/Solid Waste Samples ... A-2

A2.1 Volatile Organic Analyses QC Limits ................ A-5

A2.2 Nonhalogenated Volatile Organic Analyses QC Limits ......... A-6

A2.3 Semivolatile Organic Analyses QC Limits .............. A-6

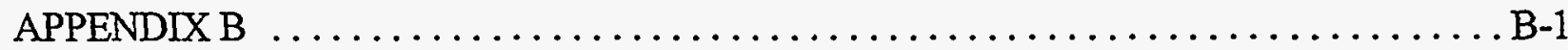

Table B1 Total Mass and Activity for Selected Species in the OHF Sludge .... B-2

Table B2 Field Dose Measurements on OHF Liquid Samples ............ B-3

Table B3 Field Dose Measurements on OHF Sludge Samples ............ B-3

Table B4 Laboratory Dose Measurements on OHF Sludge $\ldots \ldots \ldots \ldots \ldots$. B-4

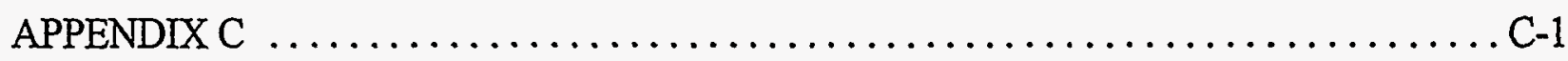

Introduction to Rheological Models for Sludge Flow Behavior ............C-1

Figure C2 Example of Rheograms for Non-Newtonian Fluids with Yield Stress . . . C-2

Figure C1 Example Rheograms for Non-Newtonian Fluids without Yield Stress . . . C-2 


\section{LIST OF TABLES}

\section{Table}

1

2

3

4

5

6

7

8

9

Summary of tanks in the Old Hydrofracture system

Page

Volume estimates for liquid and sludge in the $\mathrm{OHF}$ system $\ldots \ldots \ldots \ldots \ldots \ldots \ldots \ldots \ldots \ldots \ldots$

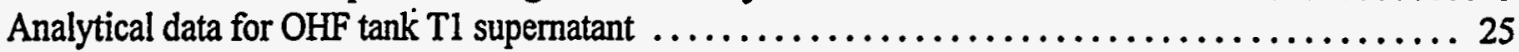

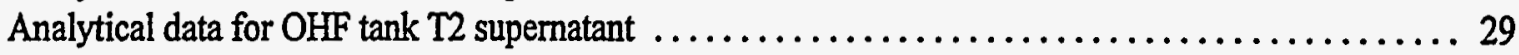

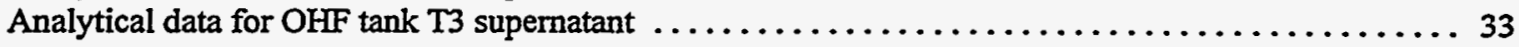

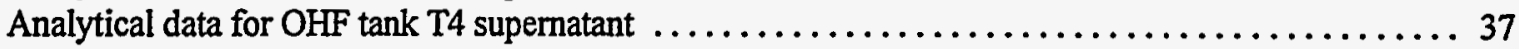

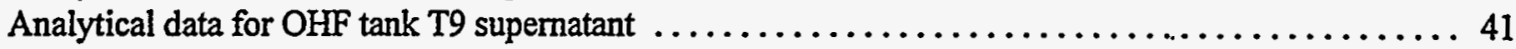

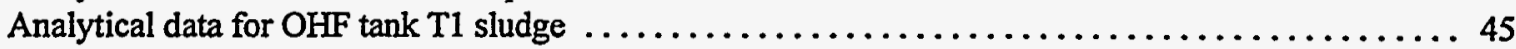

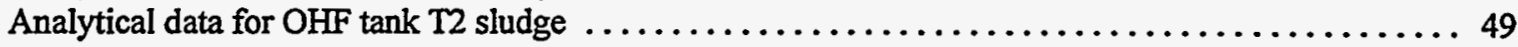

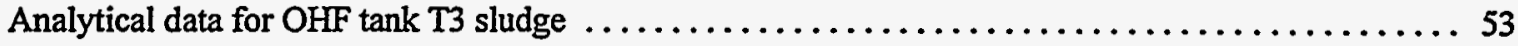

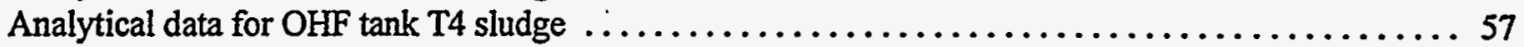

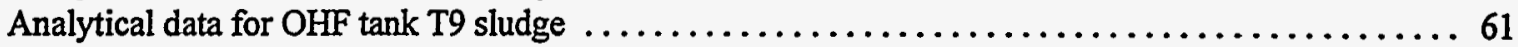

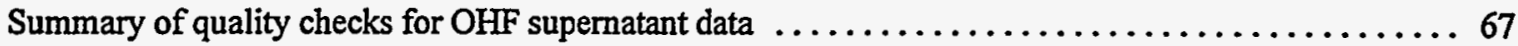

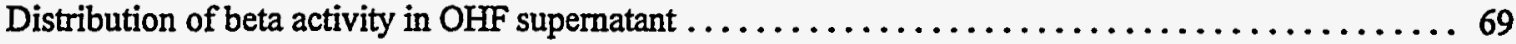

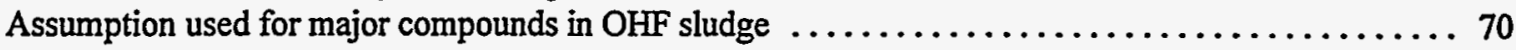

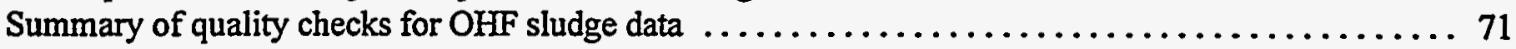

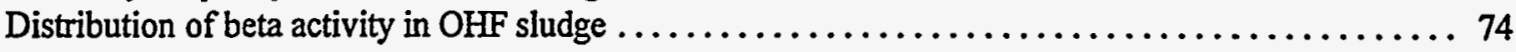

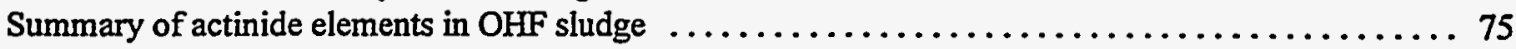

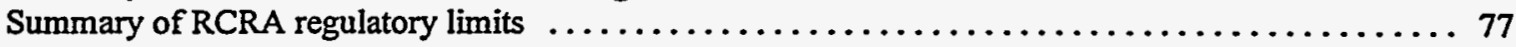

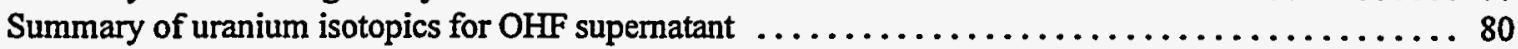

Summary of denature ratios for OHF supernatant $\ldots \ldots \ldots \ldots \ldots \ldots \ldots \ldots \ldots \ldots \ldots \ldots \ldots \ldots \ldots \ldots$

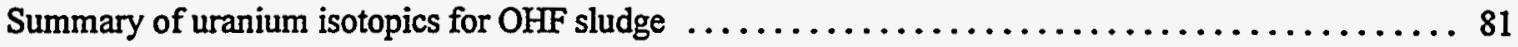

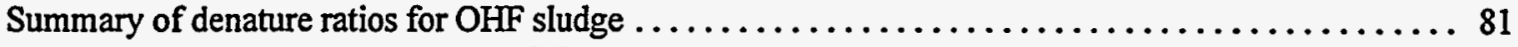

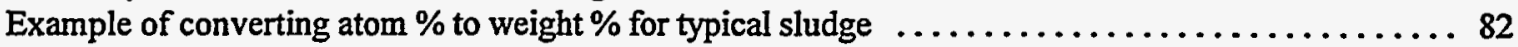

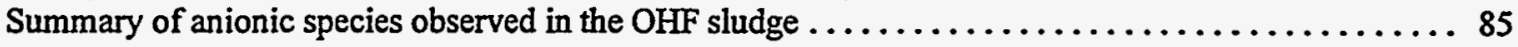

Recovery of selected species in water leach of the OHF sludge $\ldots \ldots \ldots \ldots \ldots \ldots \ldots \ldots \ldots \ldots$

Estimates for ${ }^{239} \mathrm{Pu}$ FGE with the OHF sludge $\ldots \ldots \ldots \ldots \ldots \ldots \ldots \ldots \ldots \ldots \ldots \ldots \ldots \ldots \ldots$

Estimates for ${ }^{239} \mathrm{Pu}$ equivalent activity with the $\mathrm{OHF}$ sludge $\ldots \ldots \ldots \ldots \ldots \ldots \ldots \ldots \ldots \ldots \ldots \ldots$

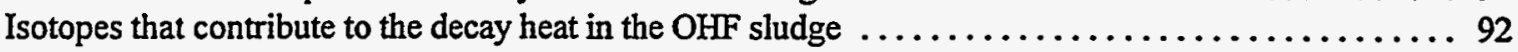

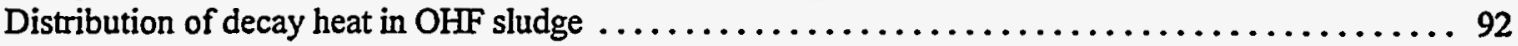

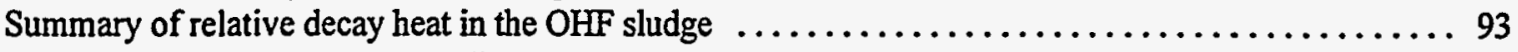

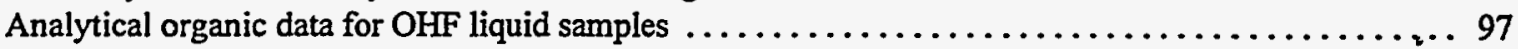

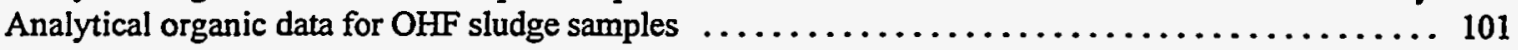

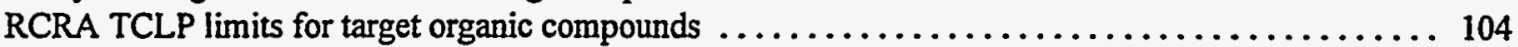

Estimation of flow properties for the OHF sludge samples $\ldots \ldots \ldots \ldots \ldots \ldots \ldots \ldots \ldots \ldots \ldots \ldots \ldots$

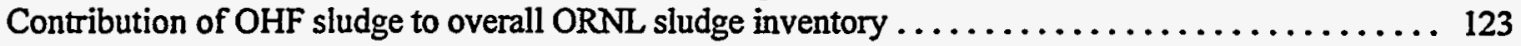

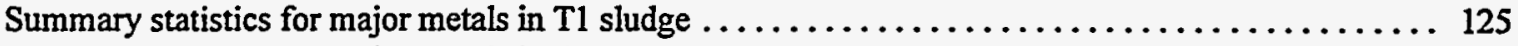

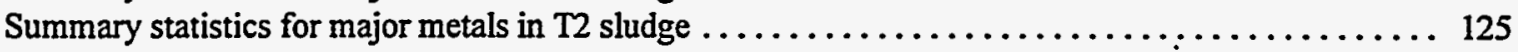

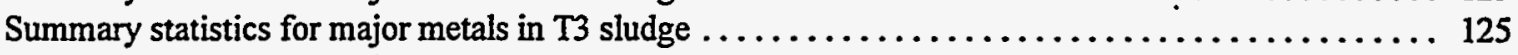

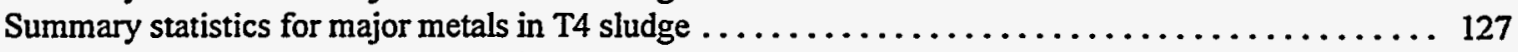

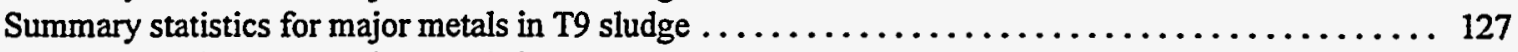

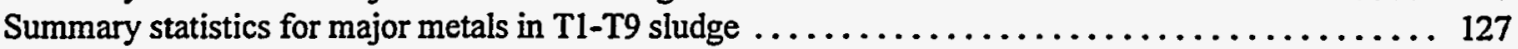

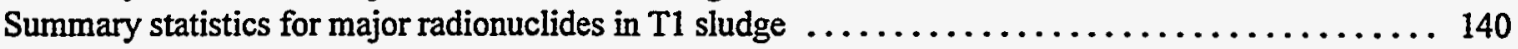

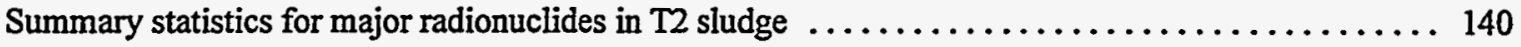

Summary statistics for major radionuclides in $\mathrm{T} 3$ sludge $\ldots \ldots \ldots \ldots \ldots \ldots \ldots \ldots \ldots \ldots \ldots \ldots \ldots$

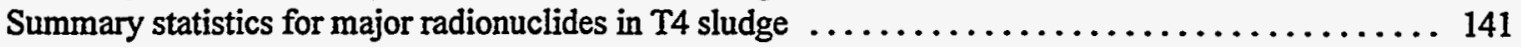

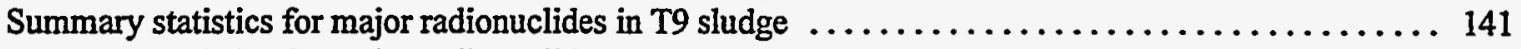

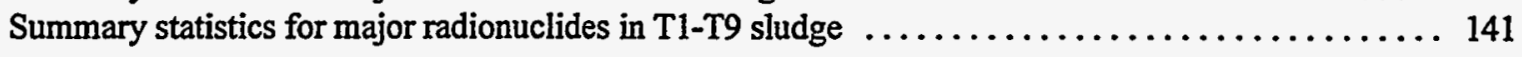




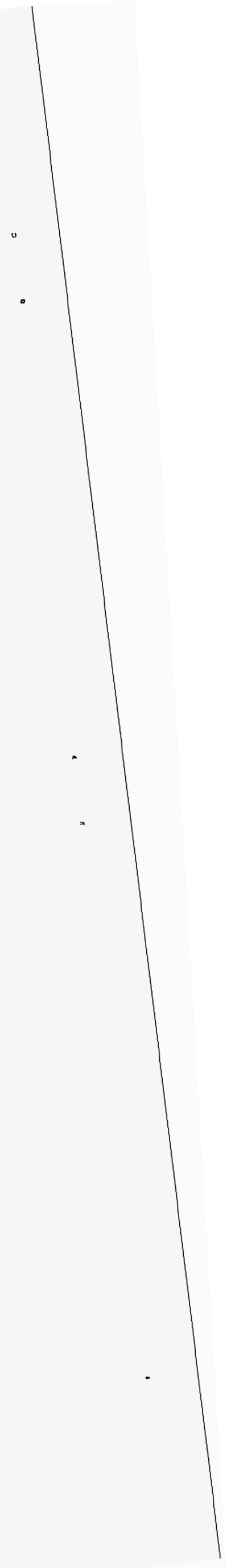




\section{LIST OF FIGURES}

\section{Figure}

Figure 1

Figure 2

Figure 3

Figure 4

Figure 5

Figure 6

Figure 7

Figure 8

Figure 9

Figure 10

Figure 11

Figure 12

Figure 13

Figure 14

Figure 15

Figure 16

Figure 17

Figure 18

Figure 19

Figure 20

Figure 21

Figure 22

Figure 23

Figure 24

Figure 25

Figure 26

Figure 27

Figure 28

Figure 29

Figure 30

Figure 31

Figure 32

Figure 33

Figure 34

Figure 35

Figure 36

Figure 37

Figure 38

Figure 39

Figure 40

Figure 41

Figure 42

Figure 43

Figure 44
Distribution of Major Cations and Anions in OHF Supernatant . . . . . . . . . . 66

Distribution of Nitrate/Nitrite Salts in OHF Supernatant $\ldots \ldots \ldots \ldots \ldots \ldots \ldots \ldots \ldots \ldots \ldots$

Distribution of Major Compounds in OHF Sludge $\ldots \ldots \ldots \ldots \ldots \ldots \ldots \ldots \ldots \ldots \ldots$

Distribution of Uranium and Thorium in OHF Sludge $\ldots \ldots \ldots \ldots \ldots \ldots \ldots \ldots$

Distribution of Major Alpha Emitters in OHF Sludge . . . . . . . . . . . . . . . . 76

Distribution of Plutonium by Alpha Activity in the OHF Sludge . . . . . . . . . . . 83

Distribution of Plutonium by Concentration in the OHF Sludge $\ldots \ldots \ldots \ldots \ldots \ldots$

Distribution of Beta Decay Heat in OHF Sludge . . . . . . . . . . . . . . . . . . . 94

Distribution of Alpha Decay Heat in OHF Sludge . . . . . . . . . . . . . . . . . . . . . 94

Shear Strength Curves for T1 Sludge Composite $\ldots \ldots \ldots \ldots \ldots \ldots \ldots \ldots \ldots \ldots \ldots \ldots \ldots 111$

Shear Strength Curves for T2 Sludge Composite $\ldots \ldots \ldots \ldots \ldots \ldots \ldots \ldots \ldots \ldots \ldots \ldots \ldots 111$

Shear Strength Curves for T3 Sludge Composite $\ldots \ldots \ldots \ldots \ldots \ldots \ldots \ldots \ldots \ldots \ldots \ldots \ldots$

Shear Strength Curves for T4 Sludge Composite . . . . . . . . . . . . . . . . . . 112

Shear Strength Curves for T9 Sludge Composite $\ldots \ldots \ldots \ldots \ldots \ldots \ldots \ldots \ldots \ldots \ldots \ldots \ldots$

Viscosity vs. Shear Rate for $\mathrm{Tl}$ Sludge Composite $\ldots \ldots \ldots \ldots \ldots \ldots \ldots \ldots \ldots \ldots \ldots \ldots$

Shear Stress vs. Shear Rate for T1 Sludge Composite $\ldots \ldots \ldots \ldots \ldots \ldots \ldots \ldots \ldots \ldots \ldots \ldots$

Viscosity vs. Shear Rate for 12 Sludge Composite . . . . . . . . . . . . . . . . . 118

Shear Stress vs. Shear Rate for $\mathrm{T} 2$ Sludge Composite $\ldots \ldots \ldots \ldots \ldots \ldots \ldots \ldots \ldots \ldots \ldots \ldots$

Viscosity vs. Shear Rate for T3 Sludge Composite . . . . . . . . . . . . . . . . . . . . . . 119

Shear Stress vs. Shear Rate for T3 Sludge Composite . . . . . . . . . . . . . . . . . . 119

Viscosity vs. Shear Rate for T4 Sludge Composite . . . . . . . . . . . . . . . . . . . . . . 120

Shear Stress vs. Shear Rate for T4 Sludge Composite . . . . . . . . . . . . . . . . . . 120

Viscosity vs. Shear Rate for T9 Sludge Composite . . . . . . . . . . . . . . . . . . 121

Shear Stress vs. Shear Rate for T9 Sludge Composite $\ldots \ldots \ldots \ldots \ldots \ldots \ldots \ldots \ldots \ldots \ldots \ldots$

Lateral Distribution of Major Metals in T1 Sludge $\ldots \ldots \ldots \ldots \ldots \ldots \ldots \ldots \ldots \ldots \ldots \ldots$

Lateral Distribution of Selected Metals in $\mathrm{T} 1$ Sludge $\quad \ldots \ldots \ldots \ldots \ldots \ldots \ldots \ldots \ldots \ldots$

Statistical Distribution of Major Metals from Lateral Sampling of T1 Sludge . . . . . . . . . . 129

Statistical Distribution of Selected Metals from Lateral Sampling of T1 Sludge . . . . . . . . 129

Lateral Distribution of Major Metals in $\mathrm{T} 2$ Sludge . . . . . . . . . . . . . . . . . 130

Lateral Distribution of Selected Metals in $\mathrm{T} 2$ Sludge $\ldots \ldots \ldots \ldots \ldots \ldots \ldots \ldots \ldots \ldots$

Statistical Distribution of Major Metals from Lateral Sampling of $\mathrm{T} 2$ Sludge . . . . . . . . 131

Statistical Distribution of Selected Metals from Lateral Sampling of T2 Sludge ........ 131

Lateral Distribution of Major Metals in T3 Sludge . . . . . . . . . . . . . . . . . 132

Lateral Distribution of Selected Metals in T3 Sludge . . . . . . . . . . . . . . . . 132

Statistical Distribution of Major Metals from Lateral Sampling of T3 Sludge . . . . . . . . 133

Statistical Distribution of Selected Metals from Lateral Sampling of T3 Sludge ......... 133

Lateral Distribution of Major Metals in T4 Sludge . . . . . . . . . . . . . . . . . . . . . 134

Lateral Distribution of Selected Metals in T4 Sludge . . . . . . . . . . . . . . . . . 134

Statistical Distribution of Major Metals from Lateral Sampling of T4 Sludge . . . . . . . . . 135

Statistical Distribution of Selected Metals from Lateral Sampling of T4 Sludge . . . . . . . 135

Lateral Distribution of Major Metals in T9 Sludge . . . . . . . . . . . . . . . 136

Lateral Distribution of Selected Metals in $\mathrm{T} 9$ Sludge $\ldots \ldots \ldots \ldots \ldots \ldots \ldots \ldots \ldots \ldots$

Statistical Distribution of Major Metals from Lateral Sampling of T9 Sludge . . . . . . . . 137

Statistical Distribution of Selected Metals from Lateral Sampling of T9 Sludge . . . . . . . . 137 


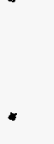




\section{ACKNOWLEDGMENTS}

The authors wish to express appreciation to the following staff members of the ORNL Chemical and Analytical Sciences Division who made important contributions to this work:

Inorganic and Radiochemical Analytical Support

L. D. Bible

R. D. Canaan

D. A. Caquelin

K. M. Hoyle

E. H. McBay

T. P. Mills ${ }^{\mathrm{a}}$

D. H. Smith

N. A. Teasley

Organic Analytical Support

S. H. Harmon

R. L. Schenley

L. T. Worthy

a Subcontractor staff, Ȯak Ridge Research Institute.

${ }^{b}$ Subcontractor staff, Midwest Technical Inc.

The authors also wish to express appreciation to the following staff members of the Liquid and Gaseous Waste Operation Department of the Office of Waste Management and Remedial Actions for tank sampling support:

\section{Sampling Radioactive Waste Tanks}
J. D. Brewer
C. B. Scott 


\section{ABBREVIATIONS AND ACRONYMS}

\begin{tabular}{|c|c|}
\hline ALARA & As Low As Reasonably Achievable \\
\hline BVEST & Bethel Valley Evaporator Service Tanks \\
\hline CAO & Carlsbad Area Office \\
\hline CASD & Chemical and Analytical Sciences Division \\
\hline CVAA & Cold Vapor Atomic Absorption \\
\hline DOT & Department of Transportation \\
\hline DQO & Data Quality Objective \\
\hline EPA & Environmental Protection Agency \\
\hline GC/MS & Gas Chromatography/Mass Spectrometry \\
\hline GC & Gas Chromatography \\
\hline GFAA & Graphite Furnace Atomic Absorption \\
\hline IC & Ion Chromatography \\
\hline ICP & Inductively Coupled Plasma \\
\hline ICP-AES & Inductively Coupled Plasma - Atomic Emission Spectroscopy \\
\hline ICP-MS & Inductively Coupled Plasma - Mass Spectrometry \\
\hline IDL & Instrument Detection Limit \\
\hline LCS & Laboratory Control Sample \\
\hline LLLW & Liquid Low-Level Waste \\
\hline LMER & Lockheed Martin Energy Research, Corp. \\
\hline LMES & Lockheed Martin Energy Systems, Inc. \\
\hline MDL & Method Detection Limit \\
\hline MS & Matrix Spike \\
\hline MSD & Matrix Spike Duplicate \\
\hline MVST & Melton Valley Storage Tanks \\
\hline NHVOA & Non-halogenated Volatile Organic Analysis \\
\hline NTS & Nevada Test Site \\
\hline ORNL & Oak Ridge National Laboratory \\
\hline PCB & Polychlorinated Biphenyls \\
\hline $\mathrm{QA}$ & Quality Assurance \\
\hline QAPjP & Quality Assurance Project Plan \\
\hline QAPP & Quality Assurance Program Plan \\
\hline QC & Quality Control \\
\hline RCRA & Resource Conservation and Recovery Act \\
\hline RMAL & Radioactive Materials Analytical Laboratory (Building 2026) \\
\hline SVOA & Semivolatile Organic Analysis \\
\hline TC & Total Carbon \\
\hline TCL & Target Compound List \\
\hline TCLP & Toxicity Characteristic Leaching Procedure \\
\hline TDS & Total Dissolved Solids \\
\hline TIC & Total Inorganic Carbon or Tentatively Identified Compounds \\
\hline TIMS & Thermal Ionization Mass Spectrometry \\
\hline TOC & Total Organic Carbon \\
\hline TRU & Transuranic \\
\hline TWCP & Transuranic Waste Characterization Program \\
\hline VOA & Volatile Organic Analysis \\
\hline UTS & Universal Treatment Standards \\
\hline WAC & Waste Acceptance Criteria \\
\hline WIPP & Waste Isolation Pilot Plant \\
\hline
\end{tabular}




\begin{abstract}
The Old Hydrofracture Facility (OHF) is located in Melton Valley within Waste Area Grouping (WAG) 5 and includes five underground storage tanks (T1, T2, T3, T4, and T9) ranging from 13,000 to 25,000 gal. capacity. During the period of 1996-97 there was a major effort to re-sample and characterize the contents of these inactive waste tanks. The characterization data summarized in this report was needed to address waste processing options, examine concerns dealing with the performance assessment (PA) data for the Waste Isolation Pilot Plant (WIPP), evaluate the waste characteristics with respect to the waste acceptance criteria (WAC) for WIPP and Nevada Test Site (NTS), address criticality concerns, and to provide the data needed to meet DOT requirements for transporting the waste. This report discusses the analytical characterization data collected on both the supernatant and sludge samples taken from three different locations in each of the OHF tanks.

The isotopic data presented in this report supports the position that fissile isotopes of uranium ${ }^{233} \mathrm{U}$ and ${ }^{235} U$ ) do not satisfy the "denature" ratios required by the administrative controls stated in the ORNL LLLW waste acceptance criteria (WAC). The fissile isotope of plutonium $\left({ }^{39} \mathrm{Pu}\right.$ and ${ }^{241} \mathrm{Pu}$ ) are diluted with thorium far above the WAC requirements. In general, the OHF sludge was found to be hazardous (RCRA) based on total metal content and the transuranic alpha activity was well above the $100 \mathrm{nCi} / \mathrm{g}$ limit for TRU waste. The characteristics of the OHF sludge relative to the WIPP WAC limits for fissile gram equivalent, plutonium equivalent activity, and thermal power from decay heat were estimated from the data in this report and found to be far below the upper boundary for any of the remote-handled transuranic waste (RH-TRU) requirements for disposal of the waste in WIPP.
\end{abstract}




\title{
Characterization of the Old Hydrofracture Facility (OHF) Waste Tanks Located at ORNL
}

\author{
J. M. Keller, J. M. Giaquinto, A. M. Meeks
}

\subsection{Introduction}

The Old Hydrofracture (OHF) Facility was built in 1963 and operated from 1964 through 1979. The purpose of the facility was to dispose of liquid waste by the hydrofracture process which involved the mixing of waste with grout followed by high pressure (3000 psi) injection of the mixture into a shale formation about 1000 feet below ground surface. The OHF Facility is located in Melton Valley within Waste Area Grouping (WAG) 5 and includes five underground storage tanks ranging from 13,000 to 25,000 gal. capacity. The OHF tanks discussed in this report are summarized in Table 1 along with some details on tank construction and active service. The characterization data summarized in this report was needed to address waste processing options, to examine concerns from the performance assessment (PA) for the Waste Isolation Pilot Plant (WIPP), to evaluate the waste characteristics with respect to the waste acceptance criteria (WAC) for WIPP and Nevada Test Site (NTS), deal with criticality concerns, and to provide data needed to meet DOT requirements for transporting the waste.

The analytical data for this report was collected during two sampling and analysis campaigns which were performed through the December 1995 - February 1996 and December 1996 - February 1997 time periods. The first sampling period included samples taken from an access port located near the center of each tank. During the summer of 1996 new access ports were installed on the north and south ends of each OHF tank, which were then used during the second sampling campaign to collect more samples from each tank. The sampling and waste characterization requirements were documented in two separate Sampling and Analysis Plans ${ }^{1-2}$ (SAP). The level of quality assurance approximates that required for regulatory measurements with the understanding that when necessary the sample size requirements were reduced, and steps were taken to reduce sample handling to ensure radiation exposures were as-low-as-reasonably-achievable (ALARA). Any deviations from procedures or problems observed with the tank samples were documented in the data files 
maintained by the laboratory. The regulatory holding time requirements for mercury and the organic measurements were complied with unless noted differently in the data tables. The Quality Control (QC) Acceptance Criteria for measurements used on this project are summarized in Appendix A. An estimate for a total inventory for selected species in each tank and both field and laboratory dose measurements on the sludge and supernatant samples are discussed in Appendix B.

Table 1 Summary of tanks in the Old Hydrofracture system

\begin{tabular}{|l|c|c|c|c|c|}
\hline \multirow{2}{*}{ Tanks } & \multirow{2}{*}{$\begin{array}{c}\text { Capacity } \\
\text { (gal) }\end{array}$} & \multirow{2}{*}{ Construction } & \multirow{2}{*}{$\begin{array}{c}\text { Active } \\
\text { Service }\end{array}$} & \multicolumn{2}{|c|}{ Data Presented in this report } \\
\cline { 5 - 6 } & & & & Liquid & Sludge \\
\hline \hline T-1 & 15000 & Mild steel & $1963-80$ & $\checkmark$ & $\checkmark$ \\
\hline T-2 & 15000 & Mild steel & $1963-80$ & $\checkmark$ & $\checkmark$ \\
\hline T-3 & 25000 & Mild Steel-RL & $1963-80$ & $\checkmark$ & $\checkmark$ \\
\hline T-4 & 25000 & Mild Steel-RL & $1963-80$ & $\checkmark$ & $\checkmark$ \\
\hline T-9 & 13000 & Mild steel & $1963-80$ & $\checkmark$ & $\checkmark$ \\
\hline
\end{tabular}

${ }^{a}$ Interior wall of tank rubber lined.

The scope of earlier OHF tank characterization work performed in $1989-90$ by Autrey ${ }^{3}$ et al. was limited to gross radiochemical measurements and RCRA metals. This earlier analytical work did not specifically address criticality concerns or many of the metals needed for process development. The radiochemical data for the fissile elements ${ }^{233} \mathrm{U},{ }^{235} \mathrm{U}$ and ${ }^{239} \mathrm{Pu}$ was estimated from gross radiochemical screening measurements. Due to the relatively large analytical errors observed with the screening measurements, any criticality control estimates based on this previous data should be used with caution. The most definitive and defensible analytical data for fissile isotopes in this report are based on thermal ionization mass spectrometry (TIMS) measurements. Additional measurements of isotopic ratios by inductively coupled - mass spectrometry (ICP-MS) are included for comparison to support this technique as a screening tool in the future. The uranium and plutonium were each chemically separated from the waste matrix prior to measurement of the isotopic ratios by TIMS. The mass spectrometry measurements yield additional detail and improved 
accuracy relative to the radiochemical measurements for the major fissile isotopes present. In general, the isotopic mass ratio measurements on a sludge sample from a single location in the tank would not represent the average isotopic ratios across the poorly mixed sludge phase commonly present in the waste tanks. Based on intermittent mixing of tank liquids with air sparging systems and the general behavior of dissolved species in liquids to equilibrate at some average concentration, the isotopic data for liquid samples should be more representative of the overall supernatant present than comparable data for the overall sludge content. Physical observations indicate that the sludge in each tank tends to be segregated into vertical layers which indicates minimal mixing of the sludge material as it was either added to the tanks or precipitated from the liquid layer. The addition of two new access manholes (on the North and South end) to each of the waste tanks, along with the center manhole sampled last year, allows a unique opportunity to evaluate lateral segregation of chemical and radiochemical species across the tank.

An inventory of radioactive liquid waste and sludge stored in each tank are listed in Table 2 and are based on volume estimates from LMES Engineering ${ }^{4}$.

Table 2 Volume estimates for liquid and sludge in the OHF system

\begin{tabular}{|c|c|c|c|c|c|c||}
\hline \multirow{2}{*}{ Tank } & \multicolumn{2}{|c|}{$\begin{array}{c}c \\
\text { Supernatant Volume }\end{array}$} & \multicolumn{2}{c|}{$\begin{array}{c}\text { Maximum } \\
\text { Sludge Volume }\end{array}$} & \multicolumn{2}{c||}{ Total Volume } \\
\cline { 2 - 7 } & (gal) & (L) & (gal) & (L) & (gal) & (L) \\
\hline \hline T-1 & 10780 & 40806. & 1410 & 5337. & 12190 & 46144. \\
T-2 & 10630 & 40238. & 1560 & 5905. & 12190 & 46144. \\
T-3 & 1960 & 7419. & 3120 & 11810. & 5080 & 19229. \\
T-4 & 14790 & 55986. & 2310 & 8744. & 17100 & 64730. \\
T-9 & 4930 & 18662. & 1140 & 4315. & 6070 & 22977. \\
\hline \hline Total & 43090 & 163113 & 9540 & 36113 & 52630 & 199226 \\
\hline
\end{tabular}




\subsection{Sample Collection Activities}

A detailed description on the sample collection techniques has been presented in previous reports and will not be discussed here (see Sections 1.3 of Reference 3). The staff from the Liquid and Gaseous Waste Operations (LGWO) Department provided all sample collection support and delivered the samples to the analytical laboratory. A current copy of these sampling procedures are available from the LGWO Department. The documentation for chain-of-custody was prepared, maintained for each sample collected, and stored with the data files by the analytical laboratory.

\subsection{Analytical Methodology}

The information and data collected from these studies are used to support various activities. The activities include demonstration of regulatory compliance, measurements to support future processing options, and data collection for risk assessments and other safety related assessments such as criticality. Standardized analytical procedures were used to the extent possible to ensure broad acceptance of the data generated. Unless stated otherwise, the U. S. Environmental Protection Agency (EPA) methods were used for the analyses of constituents listed as hazardous under the Resource Conservation and Recovery Act (RCRA), which includes all the inorganic and organic measurements presented in this report. In general the EPA Guidance Manual, Test Methods for Evaluating Solid Waste 5 (SW-846), was used for the inorganic and organic measurements. Some modifications of the standard procedures were necessary to handle the high radiation levels and the high salt/solids content of the samples. Some procedure modifications were required to generate valid data; these changes were usually needed to correct for chemical or other matrix related interferences common to DOE generated liquid waste from nuclear processes. All deviations from the standard procedures are documented in the raw data files and can be provided upon request to data users. 


\subsection{Sample Preparation}

The aqueous supernatant samples from the waste tanks were filtered or centrifuged to remove suspended particles. The clarified liquids were then digested by the SW-846 Method 3015, Microwave Assisted Acid Digestion of Aqueous Samples and Extracts. This sample preparation for aqueous samples was then used for all subsequent metal analyses by ICP-AES and GFAA and most of the radiochemical analyses. Results from a collaborative study ${ }^{6}$ with Argonne National Laboratory - East (ANL-E) demonstrated that the microwave sample digestion Method 3015/3051 provided excellent recovery for mercury. Therefore, to reduce sampling handling (ALARA) the analytical laboratory used microwave digestion method to prepare the liquid and sludge samples for mercury determination throughout this project.

The primary method for digesting the sludge samples was SW-846 Method 3051, Microwave Assisted Acid Digestion of Sediments, Sludges, Soils, and Oils. This sample preparation is considered to be a total digestion for metals by regulatory agencies and has yielded good results for most radionuclides of interest in the past. This nitric acid digestion gave poor performance on two of the target analytes, silver and silicon. Although nitric acid is excellent for dissolving silver compounds, there is usually enough chloride present in waste samples to form an insoluble silver chloride ( $\mathrm{AgCl}$ ) precipitate. If the chloride concentration is increased sufficiently, a silver chloride complex $\left(\mathrm{AgCl}_{3}^{-2}\right)$ forms which is soluble in the aqueous environment. Improved matrix spike recovery and defensible data for silver were obtained using a separate sample digestion with a high chloride concentration as discussed later in this report.

If the total silicon content in the sludge must be known to develop waste treatment options such as vitrification or grouting, another sample digestion is required. A simple nitric acid treatment will not dissolve most siliceous materials. The SW-846 Method 3052, Microwave Assisted Acid Digestion of Siliceous and Organically Based Matrices, provides the necessary digestion chemistry to yield good silicon data. Sludge samples were prepared for measurement of total silicon, by taking approximately $0.5 \mathrm{~g}$ of sludge and mixing with $7 \mathrm{~mL}$ of concentrated nitric acid and $3 \mathrm{~mL}$ of hydrofluoric acid in a fluorocarbon microwave vessel. The samples were digested for 10 minutes at $95 \%$ full power ( 570 watts) and then cooled to room temperature. The acid solution was then 
treated with excess boric acid and heated to $80^{\circ} \mathrm{C}$ for ten minutes to complex any free fluoride. This digestion mixture was cooled, filtered into a $50 \mathrm{~mL}$ volumetric flask, and diluted to volume with distilled/deionized water. Care must be exercised to ensure the digestion solution is cooled to room temperature prior to opening the sealed microwave vessel or there may be a significant loss of the volatile $\mathrm{SiF}_{4}$. The free fluoride is complexed with the boron to protect the sample introduction system to the ICP-AES and to prevent a high silicon background from the instrument glassware. The sample digestion with hydrofluoric acid should not be used directly for radiochemical measurements, especially for the measurement of lanthanides or actinides.

Most of the metal and radionuclide data presented in this report are based upon a Method 3051 digestion with approximately a 0.5 gram sludge sample and $10 \mathrm{~mL}$ of concentrated nitric acid. After the microwave digestion is completed and the solution cooled to room temperature, the sample is filtered into a volumetric flask and diluted to $50 \mathrm{~mL}$ with distilled/deionized water. To ensure valid silver and antimony data, samples were digested in a similar manner except the $10 \mathrm{~mL}$ of nitric acid was replaced with $6 \mathrm{~mL}$ of concentrated nitric acid plus $4 \mathrm{~mL}$ of concentrated hydrochloric acid. Any residue remaining after the nitric acid or nitric-hydrochloric acid digestion consisted of mostly $\mathrm{SiO}_{2}$ and was discarded.

There are several chemical forms of uranium and plutonium (also applies to other actinides) that are difficult to dissolve with nitric acid. These compounds resistant to dissolution with nitric acid include refractory oxides and frequently the metallic form of the actinide elements (good example is metallic $\mathrm{Th}$ and $\mathrm{Pu}$ ). It would be very rare and highly unlikely to find metallic plutonium and/or "high fired" plutonium oxides in the ORNL liquid waste streams. The refractory actinide oxides are much more likely to be present in environmental soil samples due to the fallout from nuclear testing ( $\mathrm{U}, \mathrm{Pu}, \mathrm{Am}$ oxides), debris waste from some nuclear processing operations, and the refractory actinide oxides from target and fuel fabrication operations (a good example is curium oxide targets used for californium production at ORNL). Refractory oxides are also a common chemical form used for the long term storage of actinide products, but these oxides are not a common chemical form for liquid waste disposal. 
The final composition of most ORNL liquid waste is a caustic sodium/potassium nitrate supernatant over a sludge consisting of mostly calcium carbonate and a mixture metal hydroxides and hydrated metal oxides. It is well known from years of nuclear processing that most of these metal hydroxides and hydrated oxides that were precipitated from basic solutions are readily soluble in nitric acid.

\subsection{Metal Analysis}

Three analytical measurement methods were used to determine all of the metals included in this report. Most of the metals are first determined by SW-846 Method 6010A, Inductively Coupled Plasma-Atomic Emission Spectroscopy (ICP-AES). There are several elements of interest for which the ICP-AES has insufficient detection limits to satisfy regulatory concerns, and these elements must be determined by Method 7000A, Atomic Absorption Methods. The Radioactive Materials Analytical Laboratory (RMAL) uses a Graphite Furnace Atomic Absorption (GFAA) Spectrometer with Zeeman background correction for elements that require better sensitivity. The elements that usually require GFAA were antimony (Method 7041), arsenic (Method 7060A), lead (Method 7421), selenium (Method 7740), and thallium (Method 7841). All the mercury measurements are done by either Method 7470A, Mercury in Liquid Waste (Manual Cold-Vapor Technique), or Method 7471A, Mercury in Solid or Semisolid Waste (Manual Cold-Vapor Technique). The samples discussed in this report were prepared for mercury analysis by the microwave technique discussed in section 3.1, the sample preparation specified in the mercury methods (7470A and $7471 \mathrm{~A})$ were not used.

The level of radioactivity in most waste tank samples required that the analytical systems used for metal measurements be modified for operation in a radiochemical hood or glove box. Custom instrument configurations are necessary to ensure contamination control and worker safety. All work was performed in radiochemical laboratories which are operated under strict radiation protection programs, with the use of protective clothing and routine contamination monitoring. Both an ICP-AES system and a GFAA system can generate dry, dusty particles which are difficult to contain and are highly hazardous when radioactive. A detailed description of the RMAL setup for these instruments are given in Appendix B of the Sears report ${ }^{7}$. 
The instrument detection limits (IDL) for various metals with undiluted aqueous samples are listed in Appendix A. For sludge samples, these detection limits must be increased by a factor that represents the sample dilution resulting from the sample preparation. For all the OHF sludge samples approximately $0.5 \mathrm{~g}$ of sample was digested and then diluted to $50 \mathrm{~mL}$ which yields approximately a 100 fold dilution for the sample, along with a 100 fold increase in the detection limits for subsequent analytical measurements.

The analytical error for the metal measurements depends upon the analytical method, the concentration level, and the chemical matrix. Inductively-coupled plasma-atomic emission spectroscopy (ICP-AES) and inductively coupled plasma-mass spectrometry (ICP-MS) are both multi-element measurement techniques that are designed for the best average performance for all elements analyzed. In general, these measurement techniques are not optimized for any single element. The sample introduction system for ICP instruments adds additional variability due to changes in sample density, viscosity, and solids content between samples and/or calibration standards. Overall, the expected analytical error for ICP measurements range from $\pm 4-6 \%$ at concentrations above 10 times the detection limit to $\pm 20-50 \%$ near the detection limit. These error estimates are typical for both ICP-AES and ICP-MS measurements.

Graphite Furnace AA instruments are generally optimized for a specific element and usually provide lower detection limits and better precision. The expected analytical error for GFAA measurements range from 3-5\% for concentrations greater than 10 times the detection limit to $20-40 \%$ near the detection limit. One advantage of GFAA analysis is that the measurements are frequently performed well above the method's detection limits. The mercury measurements were done by Cold Vapor Atomic Absorption. (CVAA), which is very selective and sensitive for mercury. The analytical errors for CVAA measurements are similar to GFAA work.

\subsection{Anion Analysis}

The determination of the inorganic anions was needed for the development of process treatment options, to provide information to explain the distribution and chemical behaviors observed in the waste tanks, and to ensure the major chemical constituents were identified in the waste for which 
data was used to calculate the mass and charge balance for each sample. The common inorganic anions; including fluoride, chloride, bromide, phosphate, nitrate, nitrite, and sulfate; were measured by ion chromatography (IC) with a Dionex Model 4500i system. In addition, several water soluble organic acids were measured along with the inorganic anions. These organic acids were measured in their ionized form and the system calibration was setup to include formate, acetate, citrate, and oxalate. Both the citrate and the oxalate can form strong complexes with many metals and change the solution chemistry of these metals in the waste. Additional anions observed in each tank are reported as tentatively identified compounds (TIC), and the reported concentrations are only estimates. The ion chromatography system used for measurements on these radioactive samples was configured such that the components that come into contact with radioactivity were isolated in a radiochemical hood for contamination control.

From past observations, the nitrate content usually dominates both the mass and charge balance calculations with both the supernatant and sludge samples taken from most of the active LLLW tanks. However, both past and current data on the OHF tanks indicates a much lower level of nitrate present in the waste tanks. In addition to the common inorganic anions, there are many other anionic species present in the waste. Many of these additional anions are measured directly by ion chromatography and others can be estimated from the metals present such as chromate, dichromate, permanganate, and others. The concentration of carbonates present in the waste samples are estimated from the total inorganic carbon (TIC) measurements.

The liquid samples were analyzed directly by ion chromatography after an appropriate dilution with water. The anion content can be used directly to account for the mass and charge balance with the aqueous samples because less assumptions must be made about the solution chemistry compared to the precipitation chemistry with the sludge samples. The mass/charge balance checks for aqueous samples should agree within the analytical error (approximately $\pm 10 \%$ ) of the measurements. The performance of balance checks for sludge samples is not expected to be as good as the liquid samples because of the large content of mixed oxides, hydrated hydroxides (heavy metals and actinides), and insoluble carbonates (calcium carbonate, etc.) present in the sludge. The complex precipitation chemistry of the sludge complicates the measurements of total anions and makes estimates for the mass and charge balance more difficult. Analytical techniques such as $\mathrm{x}$-ray 
fluorescence $(X R F)$ are useful for solid samples but are limited to total element measurements (total sulfur vs. sulfate, total phosphorus vs. phosphate). Another technique, $x$-ray diffraction (XRD), is useful for the identification of compounds present but only provides qualitative information such as the determination of crystal structures. For this report, the primary sludge anion data is based on a water leach which represents the sum of the anions in the interstitial liquid and the water soluble anions from the solids. For these measurements the sludge samples were prepared by adding approximately 1 gram of sludge to $10 \mathrm{~mL}$ of water, mixing for several minutes at room temperature on a vortex mixer, and separating the solids. The resulting solution was analyzed by ion chromatography, and the anion concentration was normalized back to the wet weight of the sludge.

Based on conversations with chemists from the Savannah River Site (SRS) and the Hanford site, who have been involved with similar waste characterization work and the experience over the past five years by the RMAL, the water leach preparation of the caustic sludge samples provides the acceptable total anion data for the halides, nitrites, nitrates, and satisfactory data for sulfate. Recent work has been done on $\mathrm{MVST}^{8}$ and $\mathrm{BVEST}^{9}$ sludge sample to resolve questions concerning the total anion content of the sludge. These studies involved several sample preparation methods which included a Parr Bomb combustion technique, a sodium peroxide/sodium hydroxide fusion, and total digestion of the sludge samples. It is important to note that these aggressive sample preparation methods for the sludge samples yield total concentrations of the element measured. An example would be sulfate analysis. A water leach of the sludge will yield a sulfate concentration due to water soluble compounds containing sulfate while a bomb, fusion and digestion preparation of the sludge would yield a sulfate concentration due not only to the compounds containing sulfates (both water soluble and insoluble) but any compound containing sulfur. In other words these preparations yield a total sulfur concentration rather than a total sulfate concentration. In theory, the same principle applies to any anion determined using the bomb, fusion, or total dissolution preparation method.

Regardless of the sample preparation method used the final anion measurement technique was ion chromatography. For simple water samples, without complex chemical matrix problems, the empirical analytical error for ion chromatography measurements ranges from 4-6\% for concentrations above 10 times the detection limits to $20-40 \%$ near the detection limit. The 
measurement of anions present at concentrations much lower $(<1 / 25)$ than other anionic species present may increase the overall error of the measurement.

\subsection{Radiochemical Analysis}

The only standard radiochemical methods useful for radioactive waste characterization are EPA Method 600/900.0, Gross Alpha and Beta Radioactivity in Drinking Water, and EPA Method 600/901.1: Gamma Emitting Radionuclides in Drinking Water. The EPA Method 600/905.0, Radioactive Strontium in Drinking Water, gave poor performance with the chemical matrix found in ORNL LLLW supernatant and sludge samples. The EPA method for gross alpha/beta measurements uses gas-flow proportional counting. In general, the proportional counting technique requires drying a sample at elevated temperatures onto a metal (usually stainless steel) plate which resulted in the loss of cesium chloride and other volatile radionuclides $\left({ }^{3} \mathrm{H},{ }^{14} \mathrm{C},{ }^{99} \mathrm{Tc},{ }^{106} \mathrm{Ru}\right.$, and $\left.{ }^{129} \mathrm{~T}\right)$ and yielded poor gross beta measurements for the ORNL waste samples. To avoid this problem, all gross beta measurements reported are based on measurements by liquid scintillation counting. Other than the gamma spectroscopy measurements, all of the radionuclide measurements were done with in-house procedures. The method detection limits for radiochemical measurements are dependent on both sample matrix and count time and are not listed here. In general, the radiochemical measurements used count times to yield at least $1 \%(10,000$ counts $)$ counting statistics. The expected errors for the radiochemical data range from $\pm 5-10 \%$ for gross alpha/beta and gamma emitter measurements to $\pm 10-20 \%$ for radionuclides that require chemical separations before counting (i.e. ${ }^{99} \mathrm{Tc},{ }^{90} \mathrm{Sr},{ }^{129} \mathrm{I}$, and ${ }^{237} \mathrm{~Np}$ ).

The long-lived fission products are typically more difficult and expensive to measure than shortlived fission products. Many of these long-lived radionuclides are either pure beta emitters or have weak, low energy, and/or low yield gamma-rays which are not very useful for accurate analytical measurements. In general, good radiochemical data requires that each of these isotopes be chemically separated from all other radioactivity prior to measurement. These chemical separations and measurements are were being done routinely for ${ }^{99} \mathrm{Tc}$ and ${ }^{129} \mathrm{I}$ because both can exist as anionic species $\left(\mathrm{TcO}_{4}^{-}, \mathrm{I}\right.$, and $\left.\mathrm{IO}_{3}^{-}\right)$in the waste, and these anions would be highly mobile in the environment. Recently, the measurements for ${ }^{129} \mathrm{I}$ have been done less frequently because of the very 
low levels observed in the active waste tanks, the relatively low risk from ${ }^{129} \mathrm{I}$, and the high cost of the analysis.

The ${ }^{99} \mathrm{Tc}$ is currently being measured by ICP-MS which is much more sensitive than counting techniques for radionuclides with a low specific activity. Earlier work on ${ }^{99} \mathrm{Tc}$ required a chemical separation prior to measurement, but recent studies have shown that the separation is not required for measurements by ICP-MS. The current ${ }^{99} \mathrm{Tc}$ measurements only require a dilution of the supernatant samples and a water leach of the sludge sample prior to analysis. Based on studies to compare the sludge water leach to a nitric acid dissolution of the sludge there was no difference between the two sample preparations which suggests the ${ }^{99} \mathrm{Tc}$ is present as the pertechnetate ion.

If measured, the ${ }^{129} \mathrm{I}$ is first extracted into carbon tetrachloride as iodine $\left(I_{2}\right)$, then reduced to iodide $\left(I^{-}\right)$, back-extracted into an aqueous matrix, and loaded onto an anion exchange resin. The ${ }^{129} \mathrm{I}$ is then determined by neutron activation analysis. Typically the level of ${ }^{99} \mathrm{Tc}$ and ${ }^{129} \mathrm{I}$ in the waste is lower than expected from the fission yields, and one possible explanation is that both isotopes may have been volatilized as $\mathrm{HTcO}_{4}, \mathrm{HI}$, and $\mathrm{I}_{2}$ when exposed to either acid and/or heat in the past.

The long-lived fission products are a very small fraction of the overall activity present in the waste, and there has been little interest in the measurement of these radionuclides in the past. The. determination of these isotopes are less routine and are frequently more expensive methods to perform. The judgement of most waste characterization teams has been that the measurement of these radionuclides, with the exception of ${ }^{99} \mathrm{Tc}$, would be interesting but there is insufficient risk to justify the analytical cost.

\subsection{Criticality Controls}

At the time samples were collected for this project, the ORNL waste acceptance criteria (WAC) for liquid-low level waste required that the fissile isotopes of uranium and plutonium be isotopically diluted with ${ }^{238} \mathrm{U}$ and ${ }^{232} \mathrm{Th}$, respectively. These administrative controls also required that the ratio of the ${ }^{238} \mathrm{U}$ mass divided by the fissile equivalent mass (FEM) for uranium be greater than 100 . The ${ }^{235} \mathrm{U} \mathrm{FEM}$ is a useful scale for criticality calculations that normalizes the fission probability for each 
fissile isotope to ${ }^{235} U$. These FEM factors, designated as $f_{35}$ for ${ }^{235} U$ mass factors, are discussed and listed in the Appendix A, Table 1 of ORNL Procedure NCS-1.0, Nuclear Criticality Safety Program.

The major fissile isotopes of concern in the ORNL waste tanks are ${ }^{233} \mathrm{U},{ }^{235} \mathrm{U}$, and ${ }^{239} \mathrm{Pu}$. The fissile isotope ${ }^{241} \mathrm{Pu}$ is also present in the waste, but the mass is usually several orders of magnitude lower and below a level that would influence the isotopic dilution ratio for plutonium. Other fissile isotopes present in the ORNL waste include isotopes of neptunium, americium, and curium, but the actual mass present in the waste has been too low for major concern, and the low concentration would make it difficult and expensive to measure by mass spectrometry.

The data presented in this report for isotopic dilution ratios (also referred to as "denature" ratios) reflect both the past and current ORNL standard practices for disposal of fissile isotopes of uranium and plutonium. The administrative controls which were in effect when the waste was generated, required that the ${ }^{233} \mathrm{U}$ and ${ }^{235} \mathrm{U}$ be diluted with depleted uranium such that the following condition was true,

$$
\frac{\left({ }^{238} U\right)}{(1.35)\left({ }^{233} U\right)+\left({ }^{235} U\right)} \geq 100
$$

Because thorium chemistry is more similar to plutonium than uranium chemistry, the administrative procedures required that the ${ }^{239} \mathrm{Pu}$ be diluted with ${ }^{232} \mathrm{Th}$ as follows,

$$
\frac{\left({ }^{232} T h\right)}{\left({ }^{239} P u\right)} \geq 100
$$

All calculations dealing with isotopic dilution for criticality safety are based on isotope mass ratios and must not be confused with activity ratios. For any data discussed in this report that uses ${ }^{232} \mathrm{Th}$ relative to isotopic mass ratios, the total thorium concentration and the ${ }^{232} \mathrm{Th}$ concentration are the same value. The new requirements for administrative criticality control, which were scheduled to be in effect by the end of 1996, are more conservative and require that the following conditions be satisfied for uranium, 


$$
\begin{aligned}
& \frac{\left.\left({ }^{238} U\right)-200{ }^{233} U\right)}{\left({ }^{235} U\right)} \geq 110 \\
& \frac{\left.\left({ }^{238} U\right)-.110{ }^{235} U\right)}{\left({ }^{233} U\right)} \geq 200
\end{aligned}
$$

The new administrative controls also change requirements for plutonium by increasing the ratio of thorium to plutonium, as given in eq. 2, from a dilution ratio of 100 to a ratio of 200.

\subsection{Organic Analysis}

The organic sample preparation and analysis methods were based on SW-846 methods which had been adapted for radioactive samples. The performance of these methods had been demonstrated according to the Transuranic Waste Characterization Program (TWCP) Quality Assurance Program Plan (QAPP) ${ }^{10}$ requirements. The amounts of sample extracted and analyzed for this project were

limited to ensure contamination control and good ALARA practices. In general, it was not necessary to reduce the sensitivities of the volatile organic compound analysis (VOA), the non-halogenated volatile organic compound analysis (NHVOA), the semivolatile organic compound analysis (SVOA), or the polychlorinated biphenyls (PCB) analysis to meet sampling handling requirements due to the radioactivity.

\subsubsection{Non-halogenated Volatile Organic Analysis (NHVOA)}

The NHVOA measurements were done by SW-846 Method 8015A, Nonhalogenated Volatile Organics by Gas Chromatography. One gram of sludge or one milliliter of supernatant was extracted by shaking with $1 \mathrm{~mL}$ of water. The sample size for this extraction was reduced two-fold from the method used in the TWCP, but the procedure retained the same method detection limit (MDL) because the relative proportions of sample and solvent were not changed. A volume of 0.001 $\mathrm{mL}$ of the extract was injected onto each of two gas chromatography columns, and the organic compounds were detected by flame ionization and quantified using the method of external standards. 
A surrogate standard was added to all samples and quality control samples. The latter included a laboratory blank, matrix spike (MS) and spike duplicate (MSD) samples, and a laboratory control sample (LCS).

\subsubsection{Volatile Organic Analysis (VOA)}

The VOA measurements were done by SW-846 Method 8260A, Volatile Organic Compounds by Gas Chromatography/Mass Spectrometry (GC/MS): Capillary Column Technique. For sludge samples $1 \mathrm{~g}$ of solids was extracted by shaking with $1 \mathrm{~mL}$ of methanol. A $0.05 \mathrm{~mL}$ aliquot of the extract was added to $5 \mathrm{~mL}$ of water and was subjected to purge and trap gas chromatography-mass spectrometry (GC-MS). For the supernatant samples, the purge and trap GC-MS was done directly on $5 \mathrm{~mL}$ of each sample. Quantitation was by the method of internal standards. Surrogate standards were added to all samples and quality control samples. The latter included a laboratory blank, MS and MSD, and a LCS.

\subsubsection{Semivolatile Organic Analysis}

The SVOA measurements included SW-846 Method 3550A, Ultrasonic Extraction, for sample preparation, and SW-846 Method 8270B, Semivolatile Organic Compounds by Gas Chromatography / Mass Spectrometry (GC/MS): Capillary Column Technique, for sample analysis. For sludge samples, $10 \mathrm{~g}$ of solids were mixed with sodium sulfate until a free-flowing matrix was obtained, and the mixture was extracted with $100 \mathrm{~mL}$ of methylene chloride using an ultrasonic bath. For supernatant samples, $200 \mathrm{~mL}$ of liquid was extracted with $100 \mathrm{~mL}$ of methylene chloride according to SW-846 Method 3510, Separatory Funnel Liquid-Liquid Extraction. The methylene chloride was concentrated to $1 \mathrm{~mL}$, and the extract was analyzed by GC-MS using the method of internal standards. Surrogate standards were added to all samples and quality control samples. The latter included a laboratory blank, MS and MSD, and a LCS.

\subsubsection{Polychlorinated Biphenyls}

The PCB measurements included SW-846 Method 3550A, Ultrasonic Extraction and Method 3665, Sulfuric Acid/Permanganate Cleanup, for sample preparation, and Method 8081, Organochlorine Pesticides and PCBs as Aroclors by Gas Chromatography: Capillary Column Technique, for sample 
analysis. A fraction of the SVOA methylene chloride extract was used for the PCB sample preparation. The extract was concentrated and solvent-exchanged into hexane, washed with sulfuric acid until the acid washes were colorless and did not contain precipitates, washed with water to remove excess acid, combined with a hexane back-extract of the acid washes, and then were concentrated to $1 \mathrm{~mL}$. Analysis was conducted on a dual capillary column GC equipped with dual electron capture detectors using the method of external standards. A surrogate standard was added to all samples and quality control samples. The latter included a laboratory blank, MS and MSD, and a LCS. 


\subsection{Quality Assurance}

Both the inorganic and organic chemical characterization of the OHF samples followed the method requirements and Data Quality Objectives (DQO) of the TWCP QAPP. The RMAL implements the TWCP QAPP with flow down to the RMAL Quality Assurance Project Plan (QAPjP) ${ }^{11}$ for the TWCP and implementation procedures. The list of metals determined was expanded from the TWCP requirements to meet ORNL needs. Although the organic target compounds were those listed in the TWCP QAPP, the full set of semivolatile and volatile organic compounds for the EPA Contract Laboratory Program Target Compound List (TCL) were reported as Tentatively Identified Compounds (TIC), if they were detected in the samples.

Quality assurance during the sampling activities was primarily addressed by the use of approved procedures for sampling both the liquid and sludge phase found in each waste tank. These procedures provide detailed instructions for the collection, labeling, and shipping of each sample. Chain-of-custody forms were used to track individual samples from their collection point to the analytical laboratory.

The RMAL also operates under a Radioactive Waste Characterization QA Plan ${ }^{12}$ which, in conjunction with the TWCP QAPjP, defines the basis for quality assurance and quality control used for the analysis of the waste tank samples. The QA plans discuss staff qualification requirements, laboratory participation in performance demonstration programs, quality control acceptance criteria for analytical methods, sample management, and most other laboratory operations. The set of QA plans implemented for RMAL waste characterization meet both the WIPP and the Nevada Test Site (NTS) QA requirements for inorganic, organic, and radiochemical measurements. 


\subsection{Summary of Inorganic and Radiochemical Analytical Results}

\subsection{Description of Data Tables}

A summary of the inorganic and radiochemical analytical results are presented in Table 3-Table 7 for the OHF supernatant samples, and the data for OHF sludge samples are presented in Table 8Table 12. These tables are arranged in a similar format to facilitate comparing data from different tanks and to group information into useful units. The analytical data presented in these tables are the consolidation of data from a single project which had a similar set of analytical requirements. Any parameter reported with a dash (" $"$ "”) indicates that the data was not measured for that sample.

The first section, "Physical properties and miscellaneous data", includes information that does not fit well into other table groups. The first parameters entered in a column include the RMAL request and sample numbers, which are laboratory filing codes used to track sample information. The sample identification is followed by the sample $\mathrm{pH}$ for liquids and the $\mathrm{pH}$ of a water leach for the sludge samples. The next set of data includes information on the solids content of the liquid samples and the moisture or water content of sludge samples along with the sample density. The group.is completed with data on the inorganic and organic carbon content. For OHF waste tank samples, the inorganic carbon can be assumed to be all carbonate and bicarbonate. The Total Organic Carbon (TOC) provides an upper limit on the organic content in the tank waste, and the current measurement methods include volatile organic compounds. However, one should recognize that most of the liquid waste in the active system has been through an evaporator prior to transfer to the OHF tanks and this evaporator system removes most of the highly volatile organic compounds from the waste.

The next two sections include groups of metals; with the "RCRA metals" separated out for quick reference. The regulatory limit for the concentrations are listed in parentheses next to each RCRA metal. For the liquid samples, the RCRA regulatory limits are used directly, since the supernatant would be defined as the TCLP leachate in the determination of hazardous waste characteristics. The RCRA metal sludge data represents total metal measurements, as defined by EPA. Exceeding the RCRA regulatory limits listed for the sludge samples only indicates that the waste has the potential 
to be classified as hazardous. The sludge waste should only be classified as RCRA waste if the final waste form fails the TCLP leaching test.

The remaining metals are grouped under "Process metals", which includes the common Group IA \& IIA metals along with elements that could effect chemical processing, criticality concerns, and stabilization techniques such as grouting or vitrification. For the sludge data, all the metals are reported on an "as received" (wet weight) basis.

The section "Semi-quantitative metals by ICP-MS" includes additional metals identified in a full mass range scan by inductively coupled plasma - mass spectrometry (ICP-MS). This measurement helps ensure all major elements have been identified in the waste. Each element reported is quantified based upon a response factor from a curve generated from a few elements across the mass range, verses quantification based upon calibration for each element of interest. Therefore, these elemental concentrations are listed as estimates only.

The "Anions by ion chromatography" section for the supernatant samples include results on the direct analysis of the sample after dilution with water. The anions reported for the sludge samples are based on a water wash of the sludge, as discussed in section 3.3. Along with the inorganic anions, several water soluble organic acids are reported, which includes compounds classified as complexing agents such as citrate and oxalate. Additional organic acid observed but not calibrated for are listed as tentatively identified compounds (TICs) similar to other organic measurements.

The "Beta/gamma emitters" section summarizes the radionuclides that emit gamma-rays and beta particles. This section includes the gross beta activity, radionuclides identified by gamma spectrometry, and may include several "pure" beta emitters of interest. Many of the "pure" beta emitters $\left({ }^{3} \mathrm{H},{ }^{14} \mathrm{C}\right.$, and $\left.{ }^{90} \mathrm{Sr}\right)$ require radiochemical separations prior to measurement by either liquid scintillation or gas-flow proportional counting. Based on recent studies the ${ }^{99} \mathrm{Tc}$ was measured by ICP-MS without any prior chemical separation. 
The "Alpha emitters" section summarize the actinide elements in the waste. These section includes the gross alpha activity, an estimate of the activity for each alpha emitter identified in a gross alpha spectrum, and plutonium isotopes determined by alpha spectrometry after a radiochemical separation, and the uranium activities are based on the TIMS data. For supernatant samples, an estimate of the ${ }^{232} \mathrm{Th} /{ }^{239} \mathrm{Pu}$ mass ratio is included in this section to address criticality concerns if enough thorium is present to calculate the ratio. For the sludge samples, this mass ratio is included with the plutonium mass spectrometry data.

The remaining sections include "Uranium isotopes by TIMS", "Plutonium isotopes by TIMS", and "Uranium isotopes by ICP-MS". These sections summarize the uranium and plutonium data measured by thermal ionization mass spectrometry (TIMS). The ICP-MS uranium measurements are included for comparison to the TIMS data to support future work that may require the quick turn around and the lower cost of ICP-MS measurements. Also, included in these sections are the isotopic mass dilution or "denature" ratios for uranium and plutonium based on the requirements in place when the waste was generated (see section 3.5). The plutonium section for the sludge samples also includes the activity for each plutonium isotope, which was calculated from the mass spectrometry data. 
Table 3 Analytical data for OHF tank T1 supernatant.

\begin{tabular}{|c|c|c|c|c|c|c|}
\hline \multicolumn{3}{|c|}{$\begin{array}{l}\text { Characteristic } \\
\text { (Analysis) }\end{array}$} & $\begin{array}{c}\text { T1-Center } \\
\text { liquid } \\
\text { (Winter 1996) }\end{array}$ & $\begin{array}{c}\text { T1-North } \\
\text { N1 liquid } \\
\text { (Winter 1997) } \\
\end{array}$ & $\begin{array}{c}\text { T1-South } \\
\text { S1 liquid } \\
\text { (Winter 1997) } \\
\end{array}$ & $\begin{array}{c}\text { T1-L35 } \\
\text { liquid } \\
\text { (ER-13 1990) } \\
\end{array}$ \\
\hline \multicolumn{7}{|c|}{ Physical properties and miscellaneous data } \\
\hline \multicolumn{3}{|c|}{$\begin{array}{ll}\text { Request number } & \\
\text { Sample number } & \\
\text { pH } & \\
\text { TSS } & (\mathrm{mg} / \mathrm{mL}) \\
\text { TDS }^{\mathbf{b}} & (\mathrm{mg} / \mathrm{mL}) \\
\text { TS } & (\mathrm{mg} / \mathrm{mL}) \\
\text { Density } & (g / \mathrm{mL}) \\
\text { TIC } & (\mathrm{mgL}) \\
\text { TC }^{d} & (\mathrm{mg} /) \\
\text { TOC }^{c} & (\mathrm{mg} /)\end{array}$} & $\begin{array}{c}7602 \mathrm{~B} \\
960123-151 \\
9.3 \\
0.02 \\
8.44 \\
8.58 \\
1.010 \\
572 \\
1050 \\
478 \\
\end{array}$ & $\begin{array}{c}7907 E \\
970107-081 \\
8.9 \\
- \\
8.1 \\
- \\
1.024 \\
600 \\
920 \\
320 \\
\end{array}$ & $\begin{array}{c}7907 \mathrm{E} \\
970107-080 \\
8.9 \\
- \\
8.1 \\
- \\
1.022 \\
610 \\
910 \\
300 \\
9\end{array}$ & $\begin{array}{l}- \\
- \\
9.7 \\
0.8 \\
6.4 \\
7.2 \\
1.005 \\
- \\
- \\
836\end{array}$ \\
\hline \multicolumn{7}{|c|}{ RCRA Metals $( \pm 10 \%)$} \\
\hline $\begin{array}{l}\mathrm{Ag} \\
\mathrm{As} \\
\mathrm{Ba} \\
\mathrm{Cd} \\
\mathrm{Cr} \\
\mathrm{Hg} \\
\mathrm{Ni} \\
\mathrm{Pb} \\
\mathrm{Se} \\
\mathrm{Tl}\end{array}$ & $\begin{array}{l}(5)^{r} \\
(5) \\
(100) \\
(1) \\
(5) \\
(0.2) \\
(50) \\
(5) \\
(1) \\
(0.9)\end{array}$ & $\begin{array}{l}(\mathrm{mg} / \mathrm{L}) \\
(\mathrm{mg} / \mathrm{L}) \\
(\mathrm{mg} / \mathrm{L}) \\
(\mathrm{mg} / \mathrm{L}) \\
(\mathrm{mg} / \mathrm{L}) \\
(\mathrm{mg} / \mathrm{L}) \\
(\mathrm{mg} / \mathrm{L}) \\
(\mathrm{mg} / \mathrm{L}) \\
(\mathrm{mg} / \mathrm{L}) \\
(\mathrm{mg} / \mathrm{L})\end{array}$ & $\begin{array}{l}<0.02 \\
0.01 \\
<0.002 \\
<0.04 \\
\quad 1.52 \\
0.544 \\
<0.04 \\
<0.008 \\
<0.008 \\
<0.008\end{array}$ & $\begin{array}{l}<0.03 \\
- \\
0.008 \\
<0.03 \\
1.33 \\
- \\
0.045 \\
- \\
- \\
-\end{array}$ & $\begin{array}{l}<0.03 \\
- \\
0.008 \\
<0.03 \\
1.33 \\
- \\
0.047 \\
- \\
-\end{array}$ & $\begin{aligned} & 0.005 \\
< & 0.8 \\
< & 0.04 \\
< & 0.02 \\
& 0.29 \\
& 0.06 \\
< & 0.2 \\
< & 1 \\
< & 0.2 \\
< & 0.2\end{aligned}$ \\
\hline \multicolumn{7}{|c|}{ Process metals ( $\pm 10 \%)$} \\
\hline $\begin{array}{l}\mathrm{Al} \\
\mathrm{B} \\
\mathrm{Be} \\
\mathrm{Bi} \\
\mathrm{Ca} \\
\mathrm{Co} \\
\mathrm{Cu} \\
\mathrm{Cs} \\
\mathrm{Fe} \\
\mathrm{K} \\
\mathrm{Mg} \\
\mathrm{Mn} \\
\mathrm{Na} \\
\mathrm{P} \\
\mathrm{Sb} \\
\mathrm{Si} \\
\mathrm{Sr} \\
\mathrm{Th} \\
\mathrm{U} \\
\mathrm{V} \\
\mathrm{Zn}\end{array}$ & & $\begin{array}{l}(\mathrm{mg} / \mathrm{L}) \\
(\mathrm{mg} / \mathrm{L}) \\
(\mathrm{mg} / \mathrm{L}) \\
(\mathrm{mg} / \mathrm{L}) \\
(\mathrm{mg} / \mathrm{L}) \\
(\mathrm{mg} / \mathrm{L}) \\
(\mathrm{mg} / \mathrm{L}) \\
(\mathrm{mg} / \mathrm{L}) \\
(\mathrm{mg} / \mathrm{L}) \\
(\mathrm{mg} / \mathrm{L}) \\
(\mathrm{mg} / \mathrm{L}) \\
(\mathrm{mg} / \mathrm{L}) \\
(\mathrm{mg} /) \\
(\mathrm{mg} / \mathrm{L}) \\
(\mathrm{mg} / \mathrm{L}) \\
(\mathrm{mg} / \mathrm{L}) \\
(\mathrm{mg} / \mathrm{L}) \\
(\mathrm{mg} / \mathrm{L}) \\
(\mathrm{mg} / \mathrm{L}) \\
(\mathrm{mg} / \mathrm{L}) \\
(\mathrm{mg} / \mathrm{L})\end{array}$ & $\begin{array}{c}0.419 \\
0.643 \\
<0.003 \\
- \\
5.78 \\
<0.02 \\
0.199 \\
0.269 \\
0.010 \\
847 \\
1.11 \\
<0.002 \\
2210 \\
48.6 \\
<0.37 \\
103 \\
0.195 \\
0.237 \\
281 \\
<0.007 \\
<0.05\end{array}$ & $\begin{array}{c}<0.06 \\
0.638 \\
<0.002 \\
5.54 \\
7.09 \\
0.045 \\
0.225 \\
- \\
<0.007 \\
706 \\
1.18 \\
0.008 \\
1940 \\
28.3 \\
<0.33 \\
20.1 \\
0.217 \\
<0.083 \\
246 \\
<0.02 \\
2.00\end{array}$ & $\begin{array}{c}<0.06 \\
0.642 \\
<0.002 \\
5.79 \\
7.26 \\
0.045 \\
0.223 \\
- \\
<0.007 \\
722 \\
1.21 \\
0.007 \\
1990 \\
27.0 \\
<0.33 \\
22.0 \\
0.222 \\
<0.083 \\
254 \\
<0.02 \\
<0.05\end{array}$ & $\begin{array}{c}- \\
- \\
- \\
- \\
\vdots \\
- \\
\vdots \\
\vdots \\
- \\
- \\
- \\
9 \\
9.34 \\
- \\
172 \\
- \\
-\end{array}$ \\
\hline
\end{tabular}




\begin{tabular}{|c|c|c|c|c|c|}
\hline $\begin{array}{l}\text { Characteristic } \\
\text { (Analysis) }\end{array}$ & & $\begin{array}{c}\begin{array}{c}\text { T1-Center } \\
\text { liquid } \\
\text { (Winter 1996) }\end{array} \\
\end{array}$ & 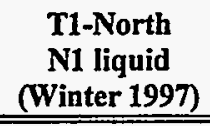 & $\begin{array}{c}\text { T1-South } \\
\text { S1 liquid } \\
\text { (Winter 1997) } \\
\end{array}$ & $\begin{array}{c}\text { T1-L35 } \\
\text { liquid } \\
\text { (ER-13 1990) } \\
\end{array}$ \\
\hline \multicolumn{6}{|c|}{ Semi-quantitative elements by ICP-MS ( $\pm 30-50 \%)$} \\
\hline $\begin{array}{l}\mathrm{Cs} \\
\mathrm{I} \\
\mathrm{Li} \\
\mathrm{Mo} \\
\mathrm{Rb} \\
\mathrm{Ru} \\
\mathrm{Sr} \\
\mathrm{Th} \\
\mathrm{Sn} \\
\mathrm{Ti} \\
\mathrm{W} \\
\mathrm{U}\end{array}$ & $\begin{array}{l}(\mathrm{mg} / \mathrm{L}) \\
(\mathrm{mg} / \mathrm{L}) \\
(\mathrm{mg} / \mathrm{L}) \\
(\mathrm{mg} / \mathrm{L}) \\
(\mathrm{mg} / \mathrm{L}) \\
(\mathrm{mg} / \mathrm{L}) \\
(\mathrm{mg} / \mathrm{L}) \\
(\mathrm{mg} / \mathrm{L}) \\
(\mathrm{mg} / \mathrm{L}) \\
(\mathrm{mg} / \mathrm{L}) \\
(\mathrm{mg} / \mathrm{L}) \\
(\mathrm{mg} / \mathrm{L})\end{array}$ & $\begin{array}{l}- \\
- \\
- \\
- \\
- \\
- \\
- \\
-\end{array}$ & $\begin{array}{l}0.10 \\
8.3 \\
1.7 \\
0.22 \\
0.14 \\
0.005 \\
0.19 \\
0.19 \\
0.11 \\
0.35 \\
0.016 \\
270\end{array}$ & $\begin{array}{l}0.13 \\
7.9 \\
1.7 \\
0.19 \\
0.15 \\
0.017 \\
0.22 \\
0.30 \\
0.083 \\
0.66 \\
0.013 \\
210\end{array}$ & $\begin{array}{l}- \\
- \\
- \\
- \\
- \\
- \\
- \\
- \\
-\end{array}$ \\
\hline \multicolumn{6}{|c|}{ Anions by ion chromatography $( \pm 10 \%)$} \\
\hline $\begin{array}{l}\text { Inorganic } \\
\text { Bromide } \\
\text { Chlorate } \\
\text { Chloride } \\
\text { Chromate } \\
\text { Fluoride } \\
\text { Nitrite } \\
\text { Nitrate } \\
\text { Phosphate } \\
\text { Sulphate } \\
\text { Organic } \\
\text { Acetate } \\
\text { Citrate } \\
\text { Formate } \\
\text { Oxalate } \\
\text { Phthalate } \\
\\
\text { Organic TICs } \\
\text { cis-Aconitate } \\
\text { trans-Aconitate } \\
\text { iso-Citrate } \\
\text { Malonate } \\
\text { Propionate }\end{array}$ & $\begin{array}{l}(\mathrm{mg} / \mathrm{L}) \\
(\mathrm{mg} / \mathrm{L}) \\
(\mathrm{mg} / \mathrm{L}) \\
(\mathrm{mg} / \mathrm{L}) \\
(\mathrm{mg} / \mathrm{L}) \\
(\mathrm{mg} / \mathrm{L}) \\
(\mathrm{mg} / \mathrm{L}) \\
(\mathrm{mg} / \mathrm{L}) \\
(\mathrm{mg} / \mathrm{L}) \\
\\
(\mathrm{mg} / \mathrm{L}) \\
(\mathrm{mg} / \mathrm{L}) \\
(\mathrm{mg} / \mathrm{L}) \\
(\mathrm{mg} / \mathrm{L}) \\
(\mathrm{mg} / \mathrm{L}) \\
\\
(\mathrm{mg} / \mathrm{L}) \\
(\mathrm{mg} / \mathrm{L}) \\
(\mathrm{mg} / \mathrm{L}) \\
(\mathrm{mg} / \mathrm{L}) \\
(\mathrm{mg} / \mathrm{L})\end{array}$ & $\begin{array}{c}<5.0 \\
- \\
464 \\
- \\
37.5 \\
948 \\
141 \\
<20 \\
557 \\
\\
- \\
- \\
- \\
- \\
- \\
\\
- \\
- \\
- \\
- \\
-\end{array}$ & $\begin{array}{c}<3 \\
1 \\
382 \\
<5 \\
36.9 \\
1450 \\
109 \\
<5 \\
473 \\
\\
\\
<3 \\
<5 \\
<3 \\
<3 \\
<5 \\
\\
\\
\text { nd } \\
\text { nd } \\
\text { nd } \\
\text { nd } \\
\text { nd }\end{array}$ & $\begin{array}{c}<3 \\
1 \\
378 \\
<5 \\
36.6 \\
1150 \\
107 \\
<5 \\
473 \\
\\
\\
<3 \\
<5 \\
<3 \\
<3 \\
<5 \\
\\
\\
\text { nd } \\
\text { nd } \\
\text { nd } \\
\text { nd } \\
\text { nd }\end{array}$ & $\begin{array}{c}- \\
- \\
7.8 \\
- \\
1.0 \\
- \\
16 \\
<5 \\
7.5 \\
\\
- \\
- \\
- \\
- \\
- \\
- \\
- \\
-\end{array}$ \\
\hline \multicolumn{6}{|c|}{ Beta/gamma emitters $( \pm 10 \%)$} \\
\hline $\begin{array}{l}\text { Gross beta } \\
{ }^{60} \mathrm{Co} \\
{ }^{134} \mathrm{Cs} \\
{ }^{137} \mathrm{Cs} /{ }^{137 m} \mathrm{Ba} \\
{ }^{152} \mathrm{Eu} \\
{ }^{154} \mathrm{Eu} \\
{ }^{155} \mathrm{Eu} \\
{ }^{90} \mathrm{Sr}{ }^{90} \mathrm{Y} \\
{ }^{99} \mathrm{Tc}\end{array}$ & $\begin{array}{l}(\mathrm{Bq} / \mathrm{mL}) \\
(\mathrm{Bq} / \mathrm{mL}) \\
(\mathrm{Bq} / \mathrm{mL}) \\
(\mathrm{Bq} / \mathrm{mL}) \\
(\mathrm{Bq} / \mathrm{mL}) \\
(\mathrm{Bq} / \mathrm{mL}) \\
(\mathrm{Bq} / \mathrm{mL}) \\
(\mathrm{Bq} / \mathrm{mL}) \\
(\mathrm{Bq} / \mathrm{mL})\end{array}$ & $\begin{array}{r}86000 \\
21 \\
<49 \\
64000 \\
<35 \\
<31 \\
<140 \\
3500 \\
13\end{array}$ & $\begin{array}{r}78000 \\
<75 \\
<170 \\
60000 \\
<370 \\
<220 \\
<440 \\
3300 \\
-\end{array}$ & $\begin{array}{r}79000 \\
<70 \\
<170 \\
61000 \\
<400 \\
<230 \\
<450 \\
3600 \\
-\end{array}$ & $\begin{array}{r}81000 \\
<50 \\
- \\
74000 \\
- \\
- \\
3300 \\
-\end{array}$ \\
\hline
\end{tabular}




\begin{tabular}{|c|c|c|c|c|c|}
\hline $\begin{array}{l}\text { Characteristic } \\
\text { (Analysis) }\end{array}$ & & 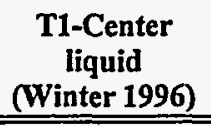 & 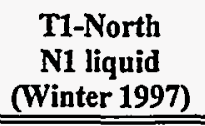 & 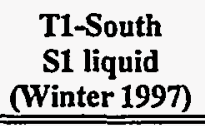 & 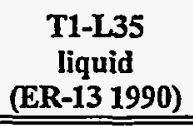 \\
\hline \multicolumn{6}{|c|}{ Alpha emitters $( \pm 10 \%)$} \\
\hline $\begin{array}{l}\text { Gross alpha } \\
{ }^{233} \mathrm{U} \\
{ }^{234} \mathrm{U} \\
{ }^{25} \mathrm{U} \\
{ }^{238} \mathrm{U} \\
\text { Total Pu alpha } \\
{ }^{238} \mathrm{Pu} \\
{ }^{239} \mathrm{Pu} /{ }^{240} \mathrm{Pu} \\
{ }^{242} \mathrm{Pu} \\
{\left[{ }^{239} \mathrm{Pu}\right]}\end{array}$ & $\begin{array}{l}(\mathrm{Bq} / \mathrm{mL}) \\
(\mathrm{Bq} / \mathrm{mL}) \\
(\mathrm{Bq} / \mathrm{mL}) \\
(\mathrm{Bq} / \mathrm{mL}) \\
(\mathrm{Bq} / \mathrm{mL}) \\
\\
(\mathrm{Bq} / \mathrm{mL}) \\
(\mathrm{Bq} / \mathrm{mL}) \\
(\mathrm{Bq} / \mathrm{mL}) \\
(\mathrm{Bq} / \mathrm{mL}) \\
(\mathrm{ng} / \mathrm{mL})\end{array}$ & $\begin{array}{c}340 \\
340 \\
6.4 \\
0.1 \\
3.5 \\
\\
2.3 \\
1.9 \\
0.4 \\
- \\
1.00 \\
237\end{array}$ & $\begin{array}{c}210 \\
300 \\
5.6 \\
0.1 \\
3.0 \\
\\
1.0 \\
0.7 \\
0.3 \\
- \\
0.12 \\
1615 \\
\end{array}$ & $\begin{array}{c}310 \\
300 \\
5.2 \\
0.1 \\
3.1 \\
\\
0.6 \\
0.4 \\
0.2 \\
- \\
0.09 \\
3443\end{array}$ & $\begin{array}{r}190 \\
180 \\
- \\
- \\
- \\
- \\
- \\
- \\
- \\
- \\
-\end{array}$ \\
\hline \multicolumn{6}{|c|}{ Uranium isotopics by TIMS $( \pm 0.5 \%)$} \\
\hline $\begin{array}{l}{ }^{233} \mathrm{U} \\
{ }^{234} \mathrm{U} \\
{ }^{23} \mathrm{U} \\
{ }^{236} \mathrm{U} \\
{ }^{28} \mathrm{U} \\
{ }^{238} \mathrm{U} / 235 \mathrm{U} F \mathrm{FE} \\
{ }^{233} \mathrm{U} \\
{ }^{235} \mathrm{U}\end{array}$ & $\begin{array}{l}\text { (atom \%) } \\
\text { (atom \%) } \\
\text { (atom \%) } \\
\text { (atom \%) } \\
\text { (atom \%) } \\
\\
(\mathrm{mg} / \mathrm{L}) \\
(\mathrm{mg} / \mathrm{L})\end{array}$ & $\begin{array}{r}0.35 \\
0.01 \\
0.56 \\
0.01 \\
99.07 \\
\\
98 \\
\\
0.96 \\
1.5\end{array}$ & $\begin{array}{l}0.345 \\
0.010 \\
0.578 \\
0.008 \\
99.059 \\
96 \\
\\
0.83 \\
1.4\end{array}$ & $\begin{array}{l}0.338 \\
0.009 \\
0.568 \\
0.008 \\
99.077 \\
\\
98 \\
\\
0.84 \\
1.4\end{array}$ & $\begin{array}{l}- \\
- \\
- \\
- \\
- \\
- \\
-\end{array}$ \\
\hline \multicolumn{6}{|c|}{ Uranium isotopics by ICP-MS $( \pm 2 \%)$} \\
\hline $\begin{array}{l}{ }^{233} U \\
{ }^{234} U \\
{ }^{235} U \\
{ }^{236} U \\
{ }^{238} U \\
{ }^{238} U /{ }^{235} U \text { FEM } \\
{ }^{233} U \\
{ }^{235} U\end{array}$ & $\begin{array}{l}\text { (atom \%) } \\
\text { (atom \%) } \\
\text { (atom \%) } \\
\text { (atom \%) } \\
\text { (atom \%) } \\
\\
(\mathrm{mg} / \mathrm{L}) \\
(\mathrm{mg} / \mathrm{L})\end{array}$ & $\begin{array}{l}- \\
- \\
- \\
- \\
- \\
- \\
-\end{array}$ & $\begin{array}{r}0.343 \\
0.010 \\
0.573 \\
0.008 \\
99.069 \\
\\
97 \\
\\
0.83 \\
1.4\end{array}$ & $\begin{array}{r}0.339 \\
0.010 \\
0.564 \\
0.008 \\
99.079 \\
99 \\
\\
0.84 \\
1.4\end{array}$ & $\begin{array}{l}- \\
- \\
- \\
- \\
- \\
-\end{array}$ \\
\hline
\end{tabular}


Table 4 Analytical data for OHF tank T2 supernatant

\begin{tabular}{|l|c|c|c|c|}
\hline $\begin{array}{l}\text { Characteristic } \\
\text { (Analysis) }\end{array}$ & $\begin{array}{c}\text { T2-Center } \\
\text { liquid } \\
\text { (Winter 1996) }\end{array}$ & $\begin{array}{c}\text { T2-North } \\
\text { N2 liquid } \\
\text { (Winter 1997) }\end{array}$ & $\begin{array}{c}\text { T2-South } \\
\text { S2 liquid } \\
\text { (Winter 1997) }\end{array}$ & $\begin{array}{c}\text { T2-L38 } \\
\text { liquid } \\
\text { (ER-13 1990) }\end{array}$ \\
\hline
\end{tabular}

Physical properties and miscellaneous data

\begin{tabular}{|c|c|c|c|c|c|}
\hline $\begin{array}{l}\text { Request number } \\
\text { Sample number } \\
\text { pH } \\
\text { TSS } \\
\text { TDS }^{b} \\
\text { TS } \\
\text { Density } \\
\text { TIC } \\
\text { TC }^{d} \\
\text { TOC }\end{array}$ & $\begin{array}{l}(\mathrm{mg} / \mathrm{mL}) \\
(\mathrm{mg} / \mathrm{mL}) \\
(\mathrm{mg} / \mathrm{mL}) \\
(\mathrm{g} / \mathrm{mL}) \\
(\mathrm{mg} / \mathrm{L}) \\
(\mathrm{mg} / \mathrm{L}) \\
(\mathrm{mg} / \mathrm{L})\end{array}$ & $\begin{array}{c}7602 \mathrm{~B} \\
960123-152 \\
9.3 \\
0.03 \\
13.5 \\
13.7 \\
1.022 \\
1060 \\
1880 \\
820\end{array}$ & $\begin{array}{c}7907 \mathrm{D} \\
970107-079 \\
9.3 \\
- \\
13.0 \\
- \\
1.034 \\
1100 \\
1600 \\
500\end{array}$ & $\begin{array}{c}7907 \mathrm{D} \\
970107-078 \\
9.3 \\
- \\
13.0 \\
- \\
1.037 \\
1100 \\
1500 \\
400\end{array}$ & $\begin{array}{c}- \\
9.4 \\
1.1 \\
11.4 \\
12.5 \\
1.013 \\
- \\
- \\
1120\end{array}$ \\
\hline
\end{tabular}

RCRA Metals ( $\pm 10 \%)$

\begin{tabular}{|c|c|c|c|c|c|c|}
\hline $\begin{array}{l}\mathrm{Ag} \\
\mathrm{As} \\
\mathrm{Ba} \\
\mathrm{Cd} \\
\mathrm{Cr} \\
\mathrm{Hg} \\
\mathrm{Ni} \\
\mathrm{Pb} \\
\mathrm{Se} \\
\mathrm{Tl}\end{array}$ & $\begin{array}{l}(5)^{t} \\
(5) \\
(100) \\
(1) \\
(5) \\
(0.2) \\
(50) \\
(5) \\
(1) \\
(0.9)\end{array}$ & $\begin{array}{l}(\mathrm{mg} / \mathrm{L}) \\
(\mathrm{mg} / \mathrm{L}) \\
(\mathrm{mg} / \mathrm{L}) \\
(\mathrm{mg} / \mathrm{L}) \\
(\mathrm{mg} / \mathrm{L}) \\
(\mathrm{mg} / \mathrm{L}) \\
(\mathrm{mg} / \mathrm{L}) \\
(\mathrm{mg} / \mathrm{L}) \\
(\mathrm{mg} / \mathrm{L}) \\
(\mathrm{mg} / \mathrm{L})\end{array}$ & $\begin{array}{c}<0.02 \\
0.009 \\
<0.002 \\
<0.04 \\
1.46 \\
0.273 \\
<0.04 \\
0.0167 \\
<0.008 \\
<0.008\end{array}$ & $\begin{array}{c}<0.03 \\
- \\
0.008 \\
<0.03 \\
1.11 \\
- \\
0.052 \\
- \\
- \\
-\end{array}$ & $\begin{array}{l}0.062 \\
- \\
0.008 \\
<0.03 \\
1.12 \\
- \\
0.063 \\
- \\
- \\
-\end{array}$ & $\begin{array}{l}<0.002 \\
<0.8 \\
<0.04 \\
<0.02 \\
\quad 0.44 \\
\quad 0.1 \\
<0.2 \\
<1 \\
<0.09 \\
<0.09\end{array}$ \\
\hline \multicolumn{7}{|c|}{ Process metals ( $\pm 10 \%)$} \\
\hline $\begin{array}{l}\mathrm{Al} \\
\mathrm{B} \\
\mathrm{Be} \\
\mathrm{Bi} \\
\mathrm{Ca} \\
\mathrm{Co} \\
\mathrm{Cu} \\
\mathrm{Cs} \\
\mathrm{Fe} \\
\mathrm{K} \\
\mathrm{Mg} \\
\mathrm{Mn} \\
\mathrm{Na} \\
\mathrm{P} \\
\mathrm{Sb} \\
\mathrm{Si} \\
\mathrm{Sr} \\
\mathrm{Th} \\
\mathrm{U} \\
\mathrm{V} \\
\mathrm{Zn}\end{array}$ & & 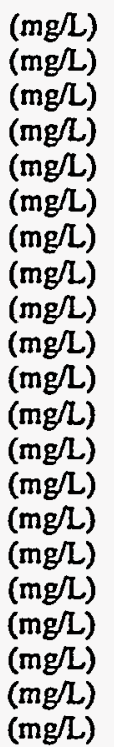 & $\begin{array}{c}0.713 \\
1.81 \\
<0.003 \\
- \\
8.98 \\
<0.02 \\
0.476 \\
19.6 \\
0.0752 \\
1380 \\
4.86 \\
<0.003 \\
3590 \\
33.4 \\
<0.39 \\
118 \\
0.139 \\
1.95 \\
219 \\
<0.007 \\
0.105\end{array}$ & $\begin{array}{c}<0.057 \\
1.65 \\
<0.002 \\
4.64 \\
10.7 \\
0.057 \\
0.485 \\
<0.007 \\
1240 \\
4.83 \\
0.022 \\
3450 \\
14.1 \\
<0.33 \\
26.3 \\
0.153 \\
1.77 \\
221 \\
<0.02 \\
0.077\end{array}$ & $\begin{array}{c}<0.057 \\
1.68 \\
<0.002 \\
4.56 \\
10.8 \\
0.047 \\
0.487 \\
- \\
<0.007 \\
1210 \\
4.93 \\
0.022 \\
3370 \\
13.9 \\
<0.33 \\
29.0 \\
0.157 \\
1.69 \\
206 \\
<0.02 \\
0.067\end{array}$ & $\begin{array}{c}- \\
- \\
- \\
- \\
- \\
\vdots \\
\vdots \\
- \\
- \\
- \\
- \\
- \\
5.07 \\
- \\
166 \\
- \\
-\end{array}$ \\
\hline
\end{tabular}




\begin{tabular}{|c|c|c|c|c|c|}
\hline $\begin{array}{l}\text { Characteristic } \\
\text { (Analysis) }\end{array}$ & & $\begin{array}{c}\begin{array}{c}\text { T2-Center } \\
\text { liquid } \\
\text { (Winter 1996) }\end{array} \\
\end{array}$ & $\begin{array}{c}\text { T2-North } \\
\text { N2 liquid } \\
\text { (Winter 1997) }\end{array}$ & $\begin{array}{c}\text { T2-South } \\
\text { S2 liquid } \\
\text { (Winter 1997) }\end{array}$ & $\begin{array}{c}\text { T2-L38 } \\
\text { liquid } \\
\text { (ER-13 1990) } \\
\end{array}$ \\
\hline \multicolumn{6}{|c|}{ Semi-quantitative elements by ICP-MS ( $\pm 30-50 \%$ ) } \\
\hline $\begin{array}{l}\mathrm{Cs} \\
\mathrm{I} \\
\mathrm{Li} \\
\mathrm{Mo} \\
\mathrm{Rb} \\
\mathrm{Ru} \\
\mathrm{Sr} \\
\mathrm{Th} \\
\mathrm{Sn} \\
\mathrm{Ti} \\
\mathrm{W} \\
\mathrm{U}\end{array}$ & $\begin{array}{l}(\mathrm{mg} / \mathrm{L}) \\
(\mathrm{mg} / \mathrm{L}) \\
(\mathrm{mg} / \mathrm{L}) \\
(\mathrm{mg} / \mathrm{L}) \\
(\mathrm{mg} / \mathrm{L}) \\
(\mathrm{mg} / \mathrm{L}) \\
(\mathrm{mg} / \mathrm{L}) \\
(\mathrm{mg} / \mathrm{L}) \\
(\mathrm{mg} / \mathrm{L}) \\
(\mathrm{mg} / \mathrm{L}) \\
(\mathrm{mg} / \mathrm{L}) \\
(\mathrm{mg} / \mathrm{L})\end{array}$ & $\begin{array}{l}- \\
- \\
- \\
- \\
- \\
- \\
- \\
- \\
-\end{array}$ & $\begin{array}{l}7.7 \\
10 \\
0.92 \\
0.25 \\
0.15 \\
0.023 \\
0.17 \\
1.5 \\
0.016 \\
0.47 \\
0.02 \\
200\end{array}$ & $\begin{array}{l}7.7 \\
9.8 \\
0.94 \\
0.24 \\
0.17 \\
0.026 \\
0.16 \\
1.0 \\
0.071 \\
0.58 \\
0.02 \\
200\end{array}$ & $\begin{array}{l}- \\
- \\
- \\
- \\
- \\
- \\
- \\
- \\
- \\
-\end{array}$ \\
\hline \multicolumn{6}{|c|}{ Anions by ion chromatography $( \pm 10 \%)$} \\
\hline $\begin{array}{l}\text { Inorganic } \\
\text { Bromide } \\
\text { Chlorate } \\
\text { Chloride } \\
\text { Chlorite } \\
\text { Chromate } \\
\text { Fluoride } \\
\text { Nitrite } \\
\text { Nitrate } \\
\text { Phosphate } \\
\text { Sulphate } \\
\text { Organic } \\
\text { Acetate } \\
\text { Citrate } \\
\text { Formate } \\
\text { Oxalate } \\
\text { Phthalate } \\
\\
\text { Organic TICs } \\
\text { cis-Aconitate } \\
\text { trans-Aconitate } \\
\text { iso-Citrate } \\
\text { Malonate } \\
\text { Propionate }\end{array}$ & $\begin{array}{l}(\mathrm{mg} / \mathrm{L}) \\
(\mathrm{mg} / \mathrm{L}) \\
(\mathrm{mg} / \mathrm{L}) \\
(\mathrm{mg} / \mathrm{L}) \\
(\mathrm{mg} / \mathrm{L}) \\
(\mathrm{mg} / \mathrm{L}) \\
(\mathrm{mg} / \mathrm{L}) \\
(\mathrm{mg} / \mathrm{L}) \\
(\mathrm{mg} / \mathrm{L}) \\
(\mathrm{mg} / \mathrm{L}) \\
\\
(\mathrm{mg} / \mathrm{L}) \\
(\mathrm{mg} / \mathrm{L}) \\
(\mathrm{mg} / \mathrm{L}) \\
(\mathrm{mg} / \mathrm{L}) \\
(\mathrm{mg} / \mathrm{L}) \\
\\
(\mathrm{mg} / \mathrm{L}) \\
(\mathrm{mg} / \mathrm{L}) \\
(\mathrm{mg} / \mathrm{L}) \\
(\mathrm{mg} / \mathrm{L}) \\
(\mathrm{mg} / \mathrm{L})\end{array}$ & $\begin{array}{c}10.4 \\
- \\
737 \\
- \\
- \\
53.4 \\
975 \\
95.2 \\
<20 \\
1380 \\
\\
- \\
- \\
- \\
- \\
- \\
- \\
- \\
- \\
- \\
-\end{array}$ & $\begin{array}{c}7.60 \\
4 \\
631 \\
5 \\
<5 \\
50.5 \\
1260 \\
<5 \\
<5 \\
1130 \\
\\
<3 \\
<5 \\
<3 \\
<3 \\
16.3 \\
\\
\text { nd } \\
\text { nd } \\
\text { nd } \\
\text { nd } \\
\text { nd }\end{array}$ & $\begin{array}{c}8.63 \\
4 \\
635 \\
5 \\
<5 \\
50.9 \\
1272 \\
<5 \\
<5 \\
1150 \\
\\
<3 \\
<5 \\
<3 \\
<3 \\
17.0 \\
\\
\text { nd } \\
\text { nd } \\
\text { nd } \\
\text { nd } \\
\text { nd }\end{array}$ & $\begin{array}{c}- \\
12 \\
- \\
- \\
1.0 \\
- \\
8.4 \\
<5 \\
22\end{array}$ \\
\hline \multicolumn{6}{|c|}{ Beta/gamma emitters $( \pm 10 \%)$} \\
\hline $\begin{array}{l}\text { Gross beta } \\
{ }^{60} \mathrm{Co} \\
{ }^{134} \mathrm{Cs} \\
{ }^{137} \mathrm{Cs} /{ }^{137} \mathrm{Ba} \\
{ }^{152} \mathrm{Eu} \\
{ }^{154} \mathrm{Eu} \\
{ }^{155} \mathrm{Eu} \\
{ }^{90} \mathrm{Sr} /{ }^{90} \mathrm{Y} \\
{ }^{90} \mathrm{Tc}\end{array}$ & $\begin{array}{l}(\mathrm{Bq} / \mathrm{mL}) \\
(\mathrm{Bq} / \mathrm{mL}) \\
(\mathrm{Bq} / \mathrm{mL}) \\
(\mathrm{Bq} / \mathrm{mL}) \\
(\mathrm{Bq} / \mathrm{mL}) \\
(\mathrm{Bq} / \mathrm{mL}) \\
(\mathrm{Bq} / \mathrm{mL}) \\
(\mathrm{Bq} / \mathrm{mL}) \\
(\mathrm{Bq} / \mathrm{mL})\end{array}$ & $\begin{array}{r}150000 \\
67 \\
<67 \\
120000 \\
<39 \\
<31 \\
<190 \\
2800 \\
20\end{array}$ & $\begin{array}{r}130000 \\
<120 \\
<220 \\
110000 \\
<110 \\
<250 \\
<560 \\
2500 \\
-\end{array}$ & $\begin{array}{r}140000 \\
<110 \\
<200 \\
110000 \\
<320 \\
<230 \\
<590 \\
2600 \\
-\end{array}$ & $\begin{array}{r}140000 \\
<75 \\
- \\
140000 \\
- \\
- \\
2500 \\
-\end{array}$ \\
\hline
\end{tabular}




\begin{tabular}{|c|c|c|c|c|c|}
\hline $\begin{array}{l}\text { Characteristic } \\
\text { (Analysis) }\end{array}$ & & $\begin{array}{c}\begin{array}{c}\text { T2-Center } \\
\text { liquid }\end{array} \\
\text { (Winter 1996) }\end{array}$ & $\begin{array}{c}\text { T2-North } \\
\text { N2 liquid } \\
\text { (Winter 1997) }\end{array}$ & $\begin{array}{c}\text { T2-South } \\
\text { S2 liquid } \\
\text { (Winter 1997) } \\
\end{array}$ & $\begin{array}{c}\text { T2-L.38 } \\
\text { liquid } \\
\text { (ER-13 1990) } \\
\end{array}$ \\
\hline \multicolumn{6}{|c|}{ Alpha emitters $( \pm 10 \%)$} \\
\hline $\begin{array}{l}\frac{\text { Gross alpha }}{{ }^{233} \mathrm{U}} \\
{ }^{234} \mathrm{U} \\
{ }^{235} \mathrm{U} \\
{ }^{23 !} \mathrm{U} \\
\text { Total Pu alpha } \\
{ }^{238} \mathrm{Pu} \\
{ }^{239} \mathrm{Pu} /{ }^{40} \mathrm{Pu} \\
{ }^{242} \mathrm{Pu} \\
\left.{ }^{239} \mathrm{Pu}\right] \\
{ }^{232} \mathrm{Th} /{ }^{39} \mathrm{Pu}(200)\end{array}$ & $\begin{array}{l}(\mathrm{Bq} / \mathrm{mL}) \\
(\mathrm{Bq} / \mathrm{mL}) \\
(\mathrm{Bq} / \mathrm{mL}) \\
(\mathrm{Bq} / \mathrm{mL}) \\
(\mathrm{Bq} / \mathrm{mL}) \\
\\
(\mathrm{Bq} / \mathrm{mL}) \\
(\mathrm{Bq} / \mathrm{mL}) \\
(\mathrm{Bq} / \mathrm{mL}) \\
(\mathrm{Bq} / \mathrm{mL}) \\
(\mathrm{ng} / \mathrm{mL})\end{array}$ & $\begin{array}{c}300 \\
270 \\
5 \\
0.1 \\
2.7 \\
\\
5.7 \\
3.3 \\
2.4 \\
- \\
2.48 \\
785\end{array}$ & $\begin{array}{c}230 \\
270 \\
4.5 \\
0.1 \\
2.7 \\
\\
5.6 \\
3.5 \\
2.1 \\
- \\
0.92 \\
1935\end{array}$ & $\begin{array}{r}220 \\
260 \\
4.2 \\
<0.1 \\
2.5 \\
\\
6.6 \\
4.3 \\
2.3 \\
- \\
1.0 \\
1687\end{array}$ & $\begin{array}{r}200 \\
190 \\
- \\
- \\
- \\
- \\
- \\
- \\
- \\
-\end{array}$ \\
\hline \multicolumn{6}{|c|}{ Uranium isotopics by TIMS $( \pm 0.5 \%)$} \\
\hline $\begin{array}{l}{ }^{233} \mathrm{U} \\
{ }^{234} \mathrm{U} \\
{ }^{235} \mathrm{U} \\
{ }^{236} \mathrm{U} \\
{ }^{238} \mathrm{U} \\
{ }^{238} \mathrm{U} /{ }^{235} \mathrm{U} \text { FEM } \\
{ }^{233} \mathrm{U} \\
{ }^{235} \mathrm{U}\end{array}$ & $\begin{array}{l}\text { (atom \%) } \\
\text { (atom \%) } \\
\text { (atom \%) } \\
\text { (atom \%) } \\
\text { (atom \%) } \\
\\
(\mathrm{mg} / \mathrm{L}) \\
(\mathrm{mg} / \mathrm{L})\end{array}$ & $\begin{array}{c}0.35 \\
0.01 \\
0.48 \\
0.01 \\
99.15 \\
106 \\
\\
0.75 \\
1.0\end{array}$ & $\begin{array}{r}0.354 \\
0.009 \\
0.497 \\
0.009 \\
99.131 \\
\\
103 \\
\\
0.77 \\
1.1\end{array}$ & $\begin{array}{r}0.354 \\
0.009 \\
0.498 \\
0.009 \\
99.130 \\
103 \\
\\
0.71 \\
1.0\end{array}$ & $\begin{array}{l}- \\
- \\
- \\
- \\
- \\
- \\
-\end{array}$ \\
\hline \multicolumn{6}{|c|}{ Uranium isotopics by ICP-MS ( $\pm 2 \%)$} \\
\hline $\begin{array}{l}{ }^{233} \mathrm{U} \\
{ }^{234} \mathrm{U} \\
{ }^{235} \mathrm{U} \\
{ }^{236} \mathrm{U} \\
{ }^{238} \mathrm{U} \\
{ }^{238} \mathrm{U} / 235 \mathrm{U} \text { FEM } \\
{ }^{233} \mathrm{U} \\
{ }^{235} \mathrm{U}\end{array}$ & $\begin{array}{l}\text { (atom \%) } \\
\text { (atom \%) } \\
\text { (atom \%) } \\
\text { (atom \%) } \\
\text { (atom \%) } \\
\\
(\mathrm{mg} / \mathrm{L}) \\
(\mathrm{mg} / \mathrm{L})\end{array}$ & $\begin{array}{l}- \\
- \\
- \\
- \\
- \\
- \\
-\end{array}$ & $\begin{array}{r}0.352 \\
0.009 \\
0.496 \\
0.009 \\
99.134 \\
104 \\
\\
0.76 \\
1.1\end{array}$ & $\begin{array}{r}0.353 \\
0.009 \\
0.494 \\
0.009 \\
99.135 \\
\\
104 \\
\\
0.71 \\
1.0\end{array}$ & $\begin{array}{l}- \\
- \\
- \\
- \\
- \\
-\end{array}$ \\
\hline
\end{tabular}


Table 5 Analytical data for OHF tank T3 supernatant

\begin{tabular}{|c|c|c|c|c|c|c|}
\hline \multicolumn{3}{|c|}{$\begin{array}{l}\text { Characteristic } \\
\text { (Analysis) }\end{array}$} & $\begin{array}{c}\begin{array}{c}\text { T3-Center } \\
\text { liquid } \\
\text { (Winter 1990) }\end{array} \\
\end{array}$ & $\begin{array}{c}\text { T3-North } \\
\text { N3 liquid } \\
\text { (Winter 1997) } \\
\end{array}$ & $\begin{array}{c}\text { T3-South } \\
\text { S3 liquid } \\
\text { (Winter 1997) } \\
\end{array}$ & $\begin{array}{c}\text { T3-LA2 } \\
\text {. liquid } \\
\text { (ER-13 1990) }\end{array}$ \\
\hline \multicolumn{7}{|c|}{ Physical properties and miscellaneous data } \\
\hline \multicolumn{2}{|c|}{$\begin{array}{l}\text { Request number } \\
\text { Sample number } \\
\text { pH } \\
\text { TSS } \\
\text { TDS } \\
\text { TS } \\
\text { Density } \\
\text { TIC }^{c} \\
\text { TC }^{d} \\
\text { TOC }^{c}\end{array}$} & $\begin{array}{l}(\mathrm{mg} / \mathrm{mL}) \\
(\mathrm{mg} / \mathrm{mL}) \\
(\mathrm{mg} / \mathrm{mL}) \\
(\mathrm{g} / \mathrm{mL}) \\
(\mathrm{mg} / \mathrm{L}) \\
(\mathrm{mg} / \mathrm{L}) \\
(\mathrm{mg} / \mathrm{L})\end{array}$ & $\begin{array}{c}7602 \mathrm{~A} \\
960117-114 \\
11.6 \\
1.46 \\
54.2 \\
56.5 \\
1.052 \\
800 \\
2930 \\
2130\end{array}$ & $\begin{array}{c}7907 \mathrm{~A} \\
961217-117 \\
9.5 \\
-\overline{47} \\
- \\
1.047 \\
1100 \\
2700 \\
1600\end{array}$ & $\begin{array}{c}7907 \mathrm{~A} \\
961217-116 \\
9.6 \\
- \\
47 \\
- \\
1.045 \\
1100 \\
2600 \\
1500\end{array}$ & $\begin{array}{c}- \\
- \\
12.7 \\
1.5 \\
51.9 \\
53.4 \\
1.035 \\
- \\
- \\
12600\end{array}$ \\
\hline \multicolumn{7}{|c|}{ RCRA Metals ( $\pm 10 \%)$} \\
\hline $\begin{array}{l}\mathrm{Ag} \\
\mathrm{As} \\
\mathrm{Ba} \\
\mathrm{Cd} \\
\mathrm{Cr} \\
\mathrm{Hg} \\
\mathrm{Ni} \\
\mathrm{Pb} \\
\mathrm{Se} \\
\mathrm{Tl}\end{array}$ & $\begin{array}{l}(5)^{f} \\
(5) \\
(100) \\
(1) \\
(5) \\
(0.2) \\
(50) \\
(5) \\
(1) \\
(0.9)\end{array}$ & $\begin{array}{l}(\mathrm{mg} / \mathrm{L}) \\
(\mathrm{mg} / \mathrm{L}) \\
(\mathrm{mg} / \mathrm{L}) \\
(\mathrm{mg} / \mathrm{L}) \\
(\mathrm{mg} / \mathrm{L}) \\
(\mathrm{mg} / \mathrm{L}) \\
(\mathrm{mg} / \mathrm{L}) \\
(\mathrm{mg} / \mathrm{L}) \\
(\mathrm{mg} / \mathrm{L}) \\
(\mathrm{mg} / \mathrm{L})\end{array}$ & $\begin{array}{c}<0.02 \\
0.298 \\
<0.002 \\
<0.04 \\
16.6 \\
12.8 \\
0.0718 \\
<0.008 \\
0.0279 \\
<0.008\end{array}$ & $\begin{array}{l}<0.03 \\
- \\
0.040 \\
<0.03 \\
15.3 \\
- \\
0.065 \\
- \\
-\end{array}$ & $\begin{array}{l}<0.03 \\
- \\
0.0433 \\
<0.03 \\
15.5 \\
- \\
0.0733 \\
- \\
-\end{array}$ & $\begin{array}{c} \\
0.01 \\
0.4 \\
<0.02 \\
<0.01 \\
14 \\
5.7 \\
<0.1 \\
<0.5 \\
<0.5 \\
<0.5\end{array}$ \\
\hline \multicolumn{7}{|c|}{ Process metals ( $\pm 10 \%)$} \\
\hline $\begin{array}{l}\mathrm{Al} \\
\mathrm{B} \\
\mathrm{Be} \\
\mathrm{Ca} \\
\mathrm{Co} \\
\mathrm{Cu} \\
\mathrm{Cs} \\
\mathrm{Fe} \\
\mathrm{K} \\
\mathrm{Mg} \\
\mathrm{Mn} \\
\mathrm{Na} \\
\mathrm{P} \\
\mathrm{Sb} \\
\mathrm{Si} \\
\mathrm{Sr} \\
\mathrm{Th} \\
\mathrm{U} \\
\mathrm{V} \\
\mathrm{Zn}\end{array}$ & & $\begin{array}{l}(\mathrm{mg} / \mathrm{L}) \\
(\mathrm{mg} / \mathrm{L}) \\
(\mathrm{mg} / \mathrm{L}) \\
(\mathrm{mg} / \mathrm{L}) \\
(\mathrm{mg} / \mathrm{L}) \\
(\mathrm{mg} / \mathrm{L}) \\
(\mathrm{mg} / \mathrm{L}) \\
(\mathrm{mg} / \mathrm{L}) \\
(\mathrm{mg} / \mathrm{L}) \\
(\mathrm{mg} / \mathrm{L}) \\
(\mathrm{mg} / \mathrm{L}) \\
(\mathrm{mg} / \mathrm{L}) \\
(\mathrm{mg} / \mathrm{L}) \\
(\mathrm{mg} / \mathrm{L}) \\
(\mathrm{mg} / \mathrm{L}) \\
(\mathrm{mg} / \mathrm{L}) \\
(\mathrm{mg} / \mathrm{L}) \\
(\mathrm{mg} / \mathrm{L}) \\
(\mathrm{mg} / \mathrm{L}) \\
(\mathrm{mg} / \mathrm{L})\end{array}$ & $\begin{array}{c}0.549 \\
4.97 \\
<0.003 \\
2.81 \\
<0.02 \\
0.0468 \\
0.569 \\
0.0217 \\
3420 \\
<0.03 \\
<0.003 \\
14800 \\
129 \\
<0.37 \\
317 \\
0.0418 \\
<0.081 \\
0.386 \\
0.424 \\
0.0551\end{array}$ & $\begin{array}{c}<0.06 \\
3.96 \\
<0.002 \\
5.49 \\
0.060 \\
0.155 \\
- \\
<0.007 \\
2860 \\
1.32 \\
0.003 \\
13300 \\
152 \\
<0.33 \\
329 \\
0.080 \\
<0.08 \\
7.72 \\
0.340 \\
<0.05\end{array}$ & $\begin{array}{c}<0.06 \\
4.06 \\
<0.002 \\
6.65 \\
0.070 \\
0.177 \\
- \\
<0.007 \\
2720 \\
1.42 \\
0.005 \\
12600 \\
150 \\
<0.33 \\
268 \\
0.085 \\
0.163 \\
7.74 \\
0.347 \\
<0.05\end{array}$ & $\begin{array}{l}- \\
- \\
- \\
- \\
- \\
- \\
- \\
- \\
- \\
- \\
- \\
7 \\
- \\
- \\
0.2 \\
- \\
-\end{array}$ \\
\hline
\end{tabular}




\begin{tabular}{|c|c|c|c|c|c|}
\hline $\begin{array}{l}\text { Characteristic } \\
\text { (Analysis) }\end{array}$ & & $\begin{array}{c}\text { T3-Center } \\
\text { liquid } \\
\text { (Winter 1996) }\end{array}$ & $\begin{array}{c}\text { T3-North } \\
\text { N3 liquid } \\
\text { (Winter 1997) }\end{array}$ & $\begin{array}{c}\text { T3-South } \\
\text { S3 liquid } \\
\text { (Winter 1997) } \\
\end{array}$ & $\begin{array}{c}\text { T3-L42 } \\
\text { liquid } \\
\text { (ER-13 1990) } \\
\end{array}$ \\
\hline \multicolumn{6}{|c|}{ Semi-quantitative elements by ICP-MS ( $\pm 30-50 \%$ ) } \\
\hline $\begin{array}{l}\mathrm{Cs} \\
\mathrm{I} \\
\mathrm{Li} \\
\mathrm{Mo} \\
\mathrm{Rb} \\
\mathrm{Ru} \\
\mathrm{Sr} \\
\mathrm{Th} \\
\mathrm{Sn} \\
\mathrm{Ti} \\
\mathrm{W} \\
\mathrm{U}\end{array}$ & $\begin{array}{l}(\mathrm{mg} / \mathrm{L}) \\
(\mathrm{mg} / \mathrm{L}) \\
(\mathrm{mg} / \mathrm{L}) \\
(\mathrm{mg} / \mathrm{L}) \\
(\mathrm{mg} / \mathrm{L}) \\
(\mathrm{mg} / \mathrm{L}) \\
(\mathrm{mg} / \mathrm{L}) \\
(\mathrm{mg} / \mathrm{L}) \\
(\mathrm{mg} / \mathrm{L}) \\
(\mathrm{mg} / \mathrm{L}) \\
(\mathrm{mg} / \mathrm{L}) \\
(\mathrm{mg} / \mathrm{L})\end{array}$ & $\begin{array}{l}- \\
- \\
- \\
- \\
- \\
- \\
- \\
-\end{array}$ & $\begin{array}{l}0.20 \\
20 \\
4.1 \\
1.0 \\
0.81 \\
0.085 \\
0.083 \\
0.36 \\
<0.001 \\
0.98 \\
0.068 \\
8.1\end{array}$ & $\begin{array}{l}0.21 \\
20 \\
4.3 \\
0.84 \\
0.83 \\
0.075 \\
0.080 \\
0.17 \\
0.026 \\
0.61 \\
0.068 \\
8.2\end{array}$ & $\begin{array}{l}- \\
- \\
- \\
- \\
- \\
- \\
- \\
-\end{array}$ \\
\hline \multicolumn{6}{|c|}{ Anions by ion chromatography $( \pm 10 \%)$} \\
\hline $\begin{array}{l}\text { Inorganic } \\
\text { Bromide } \\
\text { Chloride } \\
\text { Chromate } \\
\text { Fluoride } \\
\text { Nitrite } \\
\text { Nitrate } \\
\text { Phosphate } \\
\text { Sulphate } \\
\text { Organic } \\
\text { Acetate } \\
\text { Citrate } \\
\text { Formate } \\
\text { Oxalate } \\
\text { Phthalate } \\
\\
\text { Organic TTCs } \\
\text { cis-Aconitate } \\
\text { trans-Aconitate } \\
\text { iso-Citrate } \\
\text { Malonate } \\
\text { Propionate } \\
\text { Unknown (9) }\end{array}$ & $\begin{array}{l}(\mathrm{mg} / \mathrm{L}) \\
(\mathrm{mg} / \mathrm{L}) \\
(\mathrm{mg} / \mathrm{L}) \\
(\mathrm{mg} /) \\
(\mathrm{mg} /) \\
(\mathrm{mg} /) \\
(\mathrm{mg} /) \\
(\mathrm{mg} / \mathrm{L}) \\
\\
\\
(\mathrm{mg} / \mathrm{L}) \\
(\mathrm{mg} / \mathrm{L}) \\
(\mathrm{mg} / \mathrm{L}) \\
(\mathrm{mg} / \mathrm{L}) \\
(\mathrm{mg} / \mathrm{L})\end{array}$ & $\begin{array}{r}25.5 \\
1630 \\
- \\
283 \\
6300 \\
7140 \\
<20 \\
4890 \\
\\
- \\
- \\
- \\
-\end{array}$ & $\begin{array}{c}18.0 \\
1360 \\
34.7 \\
188 \\
10100 \\
5900 \\
<10 \\
3820 \\
\\
\\
737 \\
<10 \\
619 \\
860 \\
<10 \\
\\
\\
\sim 1 \\
\sim 1 \\
\sim 1 \\
\sim 25 \\
\sim 50 \\
\sim 50\end{array}$ & $\begin{array}{c}25.9 \\
1400 \\
38.4 \\
196 \\
10200 \\
6080 \\
<10 \\
3900 \\
\\
\\
748 \\
<10 \\
630 \\
880 \\
<10 \\
\\
\\
\sim 1 \\
\sim 1 \\
\sim 1 \\
\sim 25 \\
\sim 50 \\
\sim 50 \\
\sim\end{array}$ & $\begin{array}{c}- \\
7.0 \\
- \\
1.8 \\
- \\
53 \\
160 \\
15 \\
\\
- \\
- \\
- \\
- \\
- \\
- \\
- \\
- \\
- \\
-\end{array}$ \\
\hline \multicolumn{6}{|c|}{ Beta/gamma emitters $( \pm 10 \%)$} \\
\hline $\begin{array}{l}\frac{\text { Gross beta }}{{ }^{60} \mathrm{Co}} \\
{ }^{144} \mathrm{Cs} \\
{ }^{137} \mathrm{Cs} /{ }^{137 m} \mathrm{Ba} \\
{ }^{152} \mathrm{Eu} \\
{ }^{154} \mathrm{Eu} \\
{ }^{155} \mathrm{Eu} \\
{ }^{90} \mathrm{Sr}{ }^{\circ 0} \mathrm{Y} \\
{ }^{99} \mathrm{Tc}\end{array}$ & $\begin{array}{l}(\mathrm{Bq} / \mathrm{mL}) \\
(\mathrm{Bq} / \mathrm{mL}) \\
(\mathrm{Bq} / \mathrm{mL}) \\
(\mathrm{Bq} / \mathrm{mL}) \\
(\mathrm{Bq} / \mathrm{mL}) \\
(\mathrm{Bq} / \mathrm{mL}) \\
(\mathrm{Bq} / \mathrm{mL}) \\
(\mathrm{Bq} / \mathrm{mL}) \\
(\mathrm{Bq} / \mathrm{mL})\end{array}$ & $\begin{array}{r}230000 \\
120 \\
<82 \\
190000 \\
<35 \\
<50 \\
<240 \\
240 \\
29\end{array}$ & $\begin{array}{r}200000 \\
87 \\
<260 \\
170000 \\
<380 \\
<150 \\
<730 \\
430 \\
-\end{array}$ & $\begin{array}{r}200000 \\
140 \\
<270 \\
170000 \\
<350 \\
<220 \\
<710 \\
530 \\
-\end{array}$ & $\begin{array}{r}280000 \\
360 \\
- \\
270000 \\
- \\
- \\
- \\
300\end{array}$ \\
\hline
\end{tabular}




\begin{tabular}{|c|c|c|c|c|}
\hline $\begin{array}{l}\text { Characteristic } \\
\text { (Analysis) }\end{array}$ & $\begin{array}{c}\begin{array}{c}\text { T3-Center } \\
\text { liquid }\end{array} \\
\text { (Winter 1996) }\end{array}$ & $\begin{array}{c}\text { T3-North } \\
\text { N3 liquid } \\
\text { (Winter,1997) }\end{array}$ & $\begin{array}{c}\text { T3-South } \\
\text { S3 liquid } \\
\text { (Winter 1997) }\end{array}$ & $\begin{array}{c}\text { T3-L42 } \\
\text { liquid } \\
\text { (ER-13 1990) }\end{array}$ \\
\hline \multicolumn{5}{|l|}{ Alpha emitters $( \pm 10 \%)$} \\
\hline $\begin{array}{ll}\text { Gross alpha } & (\mathrm{Bq} / \mathrm{mL}) \\
{ }^{233} \mathrm{U} & (\mathrm{Bq} / \mathrm{mL}) \\
{ }^{234} \mathrm{U} & (\mathrm{Bq} / \mathrm{mL}) \\
{ }^{235} \mathrm{U} & (\mathrm{Bq} / \mathrm{mL}) \\
{ }^{28} \mathrm{U} & \\
& \\
\text { Total Pu alpha } & (\mathrm{Bq} / \mathrm{mL}) \\
{ }^{238} \mathrm{Pu} & (\mathrm{Bq} / \mathrm{mL}) \\
{ }^{239} \mathrm{Pu} /{ }^{40} \mathrm{Pu} & (\mathrm{Bq} / \mathrm{mL}) \\
{ }^{242} \mathrm{Pu} & (\mathrm{Bq} / \mathrm{mL}) \\
\left.{ }^{230} \mathrm{Pu}\right] & (\mathrm{ng} / \mathrm{mL})\end{array}$ & $\begin{array}{r}3.1 \\
0.3 \\
-\quad 0 \\
0 \\
0 \\
\\
0.43 \\
- \\
- \\
- \\
0.19 \\
-\end{array}$ & $\begin{array}{r}5.8 \\
5.1 \\
0.1 \\
<0.1 \\
0.1 \\
\\
0.10 \\
0.03 \\
0.07 \\
- \\
0.03 \\
11800\end{array}$ & $\begin{array}{r}3.2 \\
4.9 \\
0.1 \\
<0.1 \\
0.1 \\
\\
0.14 \\
0.05 \\
0.09 \\
- \\
0.04 \\
4160\end{array}$ & $\begin{array}{l}2 \\
2 \\
- \\
- \\
- \\
- \\
- \\
- \\
- \\
-\end{array}$ \\
\hline \multicolumn{5}{|c|}{ Uranium isotopics by TIMS $( \pm 0.5 \%)$} \\
\hline $\begin{array}{ll}{ }^{233} \mathrm{U} & \text { (atom \%) } \\
{ }^{234} \mathrm{U} & \text { (atom \%) } \\
{ }^{235} \mathrm{U} & \text { (atom \%) } \\
{ }^{236} \mathrm{U} & \text { (atom \%) } \\
{ }^{233} \mathrm{U} & \text { (atom \%) } \\
{ }^{235} \mathrm{U} /{ }^{235} \mathrm{U} \text { FEM } & \\
{ }^{233} \mathrm{U} & (\mathrm{mg} / \mathrm{L}) \\
{ }^{235} \mathrm{U} & (\mathrm{mg} / \mathrm{L})\end{array}$ & $\begin{array}{l}0.22 \\
0.01 \\
0.57 \\
0.01 \\
99.19 \\
116 \\
\\
0.0008 \\
0.002\end{array}$ & $\begin{array}{r}0.188 \\
0.008 \\
0.614 \\
0.008 \\
99.182 \\
116 \\
\\
0.014 \\
0.047\end{array}$ & $\begin{array}{r}0.182 \\
0.008 \\
0.616 \\
0.008 \\
99.186 \\
117 \\
\\
0.014 \\
0.047\end{array}$ & $\begin{array}{l}- \\
- \\
- \\
- \\
- \\
-\end{array}$ \\
\hline \multicolumn{5}{|c|}{ Uranium isotopics by ICP-MS $( \pm 2 \%)$} \\
\hline $\begin{array}{ll}{ }^{233} \mathrm{U} & \text { (atom \%) } \\
{ }^{234} \mathrm{U} & \text { (atom \%) } \\
{ }^{235} \mathrm{U} & \text { (atom \%) } \\
{ }^{236} \mathrm{U} & \text { (atom \%) } \\
{ }^{238} \mathrm{U} & \text { (atom \%) } \\
{ }^{238} \mathrm{U} /{ }^{235} \mathrm{U} \text { FEM } & \\
{ }^{233} \mathrm{U} & (\mathrm{mg} / \mathrm{L}) \\
{ }^{235} \mathrm{U} & (\mathrm{mg} / \mathrm{L})\end{array}$ & $\begin{array}{l}- \\
- \\
- \\
- \\
- \\
-\end{array}$ & $\begin{array}{r}0.167 \\
0.005 \\
0.605 \\
0.007 \\
99.216 \\
115 \\
\\
0.013 \\
0.048\end{array}$ & $\begin{array}{r}0.192 \\
0.007 \\
0.620 \\
0.008 \\
99.175 \\
115 \\
\\
0.015 \\
0.047\end{array}$ & $\begin{array}{l}- \\
- \\
- \\
- \\
- \\
- \\
-\end{array}$ \\
\hline
\end{tabular}


Table 6 Analytical data for OHF tank T4 supernatant

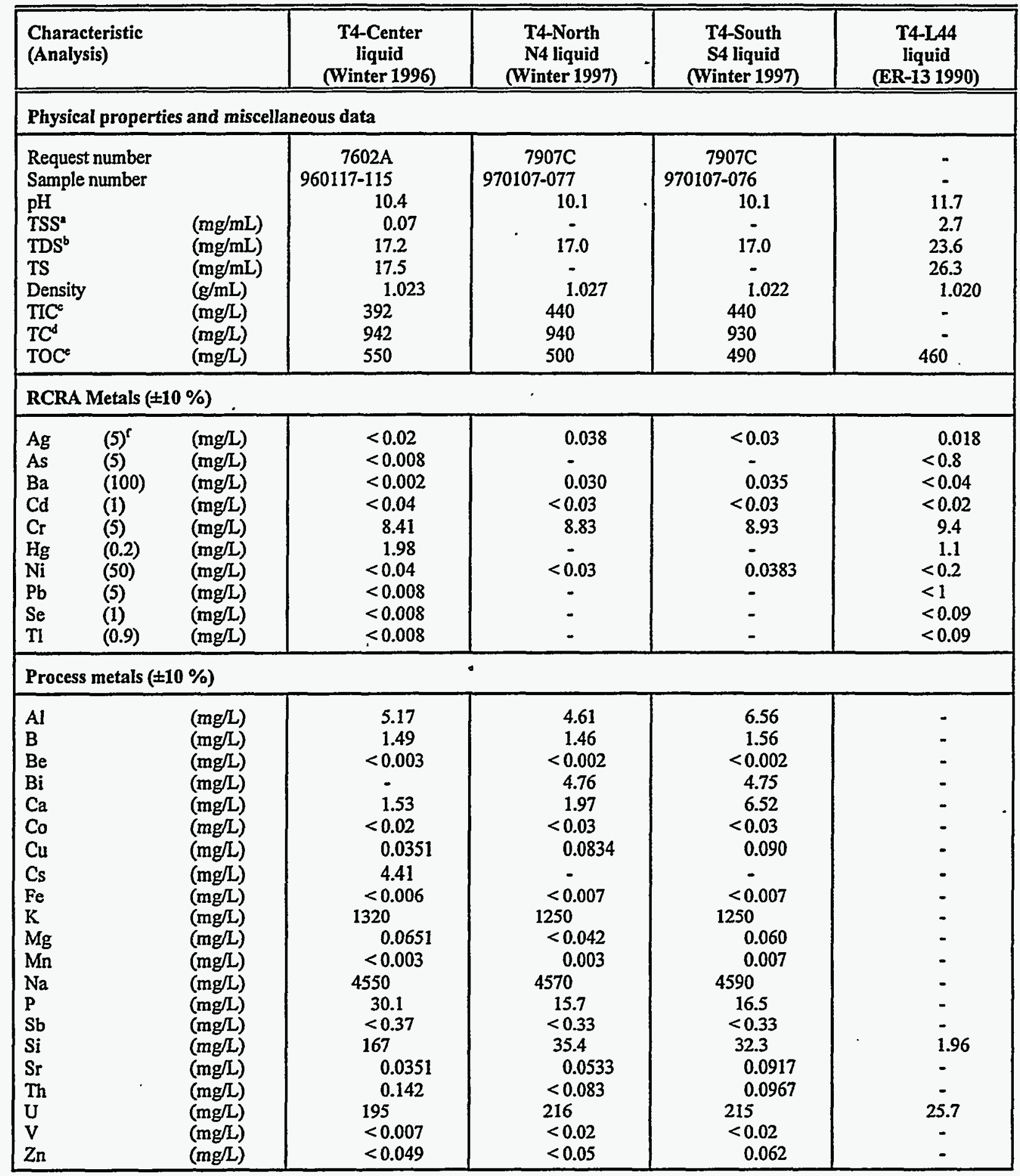




\begin{tabular}{|c|c|c|c|c|c|}
\hline $\begin{array}{l}\text { Characteristic } \\
\text { (Analysis) }\end{array}$ & & $\begin{array}{c}\text { T4-Center } \\
\text { liquid } \\
\text { (Winter 1996) } \\
\end{array}$ & $\begin{array}{c}\text { T4-North } \\
\text { N4 liquid } \\
\text { (Winter 1997) } \\
\end{array}$ & $\begin{array}{c}\text { T4-South } \\
\text { S4 liquid } \\
\text { (Winter 1997) } \\
\end{array}$ & $\begin{array}{c}\text { T4-L44 } \\
\text { liquid } \\
\text { (ER-13 1990) } \\
\end{array}$ \\
\hline \multicolumn{6}{|c|}{ Semi-quantitative elements by ICP-MS ( $\pm 30-50 \%)$} \\
\hline $\begin{array}{l}\mathrm{Cs} \\
\mathrm{I} \\
\mathrm{Li} \\
\mathrm{Mo} \\
\mathrm{Rb} \\
\mathrm{Ru} \\
\mathrm{Sr} \\
\mathrm{Th} \\
\mathrm{Sn} \\
\mathrm{Ti} \\
\mathrm{W} \\
\mathrm{U}\end{array}$ & $\begin{array}{l}(\mathrm{mg} / \mathrm{L}) \\
(\mathrm{mg} / \mathrm{L}) \\
(\mathrm{mg} / \mathrm{L}) \\
(\mathrm{mg} / \mathrm{L}) \\
(\mathrm{mg} / \mathrm{L}) \\
(\mathrm{mg} / \mathrm{L}) \\
(\mathrm{mg} / \mathrm{L}) \\
(\mathrm{mg} / \mathrm{L}) \\
(\mathrm{mg} / \mathrm{L}) \\
(\mathrm{mg} / \mathrm{L}) \\
(\mathrm{mg} / \mathrm{L}) \\
(\mathrm{mg} / \mathrm{L})\end{array}$ & $\begin{array}{l}- \\
- \\
- \\
- \\
- \\
- \\
- \\
-\end{array}$ & $\begin{array}{l}1.8 \\
17 \\
0.12 \\
0.36 \\
0.24 \\
0.024 \\
0.035 \\
0.11 \\
0.031 \\
0.77 \\
0.006 \\
210\end{array}$ & $\begin{array}{l}1.8 \\
12 \\
0.12 \\
0.31 \\
0.22 \\
0.025 \\
0.085 \\
0.16 \\
0.025 \\
0.35 \\
0.010 \\
220\end{array}$ & $\begin{array}{l}- \\
- \\
- \\
- \\
- \\
- \\
- \\
-\end{array}$ \\
\hline \multicolumn{6}{|c|}{ Anions by ion chromatography $( \pm 10 \%)$} \\
\hline $\begin{array}{l}\text { Inorganic } \\
\text { Bromide } \\
\text { Chloride } \\
\text { Chromate } \\
\text { Fluoride } \\
\text { Nitrite } \\
\text { Nitrate } \\
\text { Phosphate } \\
\text { Sulphate } \\
\text { Organic } \\
\text { Acetate } \\
\text { Citrate } \\
\text { Formate } \\
\text { Oxalate } \\
\text { Phthalate } \\
\\
\text { Organic TICs } \\
\text { cis-Aconitate } \\
\text { trans-Aconitate } \\
\text { iso-Citrate } \\
\text { Malonate } \\
\text { Propionate } \\
\text { Unknown (2) }\end{array}$ & $\begin{array}{l}(\mathrm{mg} / \mathrm{L}) \\
(\mathrm{mg} / \mathrm{L}) \\
(\mathrm{mg} / \mathrm{L}) \\
(\mathrm{mg} / \mathrm{L}) \\
(\mathrm{mg} / \mathrm{L}) \\
(\mathrm{mg} / \mathrm{L}) \\
(\mathrm{mg} / \mathrm{L}) \\
(\mathrm{mg} / \mathrm{L}) \\
\\
(\mathrm{mg} / \mathrm{L}) \\
(\mathrm{mg} / \mathrm{L}) \\
(\mathrm{mg} / \mathrm{L}) \\
(\mathrm{mg} / \mathrm{L}) \\
(\mathrm{mg} / \mathrm{L}) \\
\\
(\mathrm{mg} / \mathrm{L}) \\
(\mathrm{mg} / \mathrm{L}) \\
(\mathrm{mg} / \mathrm{L}) \\
(\mathrm{mg} / \mathrm{L}) \\
(\mathrm{mg} / \mathrm{L}) \\
(\mathrm{mg} / \mathrm{L})\end{array}$ & $\begin{array}{c}11.8 \\
650 \\
- \\
59.2 \\
1680 \\
3010 \\
<20 \\
1580\end{array}$ & $\begin{array}{c}<5 \\
493 \\
<10 \\
56.2 \\
2970 \\
2840 \\
<10 \\
1470 \\
\\
\\
285 \\
<10 \\
249 \\
453 \\
<10 \\
\\
\\
\text { nd } \\
\text { nd } \\
\text { nd } \\
\text { nd } \\
20 \\
10\end{array}$ & $\begin{array}{c}<5 \\
584 \\
14.1 \\
66.0 \\
2970 \\
2850 \\
<10 \\
1490 \\
\\
\\
295 \\
<10 \\
244 \\
448 \\
<10 \\
\\
\\
\text { nd } \\
\text { nd } \\
\text { nd } \\
\text { nd } \\
20 \\
10\end{array}$ & $\begin{array}{c}- \\
3.1 \\
- \\
1.1 \\
26 \\
51 \\
6.3 \\
- \\
- \\
- \\
- \\
- \\
- \\
- \\
-\end{array}$ \\
\hline \multicolumn{6}{|c|}{ Beta/gamma emitters $( \pm 10 \%)$} \\
\hline $\begin{array}{l}\text { Gross beta } \\
{ }^{60} \mathrm{Co} \\
{ }^{134} \mathrm{Cs} \\
{ }^{137} \mathrm{Cs} /{ }^{137 m} \mathrm{Ba} \\
{ }^{152} \mathrm{Eu} \\
{ }^{154} \mathrm{Eu} \\
{ }^{155} \mathrm{Eu} \\
{ }^{90} \mathrm{Sr}^{90} \\
{ }^{95} \mathrm{TC}\end{array}$ & $\begin{array}{l}(\mathrm{Bq} / \mathrm{mL}) \\
(\mathrm{Bq} / \mathrm{mL}) \\
(\mathrm{Bq} / \mathrm{mL}) \\
(\mathrm{Bq} / \mathrm{mL}) \\
(\mathrm{Bq} / \mathrm{mL}) \\
(\mathrm{Bq} / \mathrm{mL}) \\
(\mathrm{Bq} / \mathrm{mL}) \\
(\mathrm{Bq} / \mathrm{mL}) \\
(\mathrm{Bq} / \mathrm{mL})\end{array}$ & $\begin{array}{r}220000 \\
<17 \\
<81 \\
180000 \\
<35 \\
<44 \\
<240 \\
1700 \\
24\end{array}$ & $\begin{array}{r}210000 \\
<130 \\
<260 \\
180000 \\
<380 \\
<220 \\
<700 \\
1700 \\
-\end{array}$ & $\begin{array}{r}210000 \\
<80 \\
<260 \\
180000 \\
<230 \\
<200 \\
<720 \\
1800 \\
-\end{array}$ & $\begin{array}{r}280000 \\
64 \\
- \\
300000 \\
- \\
- \\
- \\
- \\
-\end{array}$ \\
\hline
\end{tabular}




\begin{tabular}{|c|c|c|c|c|c|}
\hline $\begin{array}{l}\text { Characteristic } \\
\text { (Analysis) }\end{array}$ & & $\begin{array}{c}\begin{array}{c}\text { T4-Center } \\
\text { liquid } \\
\text { (Winter 1996) }\end{array} \\
\end{array}$ & $\begin{array}{c}\text { T4-North } \\
\text { N4 liquid } \\
\text { (Winter 1997) }\end{array}$ & $\begin{array}{c}\text { T4-South } \\
\text { S4 liquid } \\
\text { (Winter 1997) } \\
\end{array}$ & $\begin{array}{c}\text { T4-L44 } \\
\text { liquid } \\
\text { (ER-13 1990) }\end{array}$ \\
\hline \multicolumn{6}{|c|}{ Alpha emitters $( \pm 10 \%)$} \\
\hline $\begin{array}{l}\text { Gross alpha } \\
\frac{{ }^{233} \mathrm{U}}{{ }^{234} \mathrm{U}} \\
{ }^{238} \mathrm{U} \\
{ }^{238} \mathrm{U} \\
\text { Total Pu alpha } \\
{ }^{238} \mathrm{Pu} \\
{ }^{230} \mathrm{Pu}{ }^{240} \mathrm{Pu} \\
{ }^{242} \mathrm{Pu} \\
\left.{ }^{230} \mathrm{Pu}\right]\end{array}$ & $\begin{array}{l}(\mathrm{Bq} / \mathrm{mL}) \\
(\mathrm{Bq} / \mathrm{mL}) \\
(\mathrm{Bq} / \mathrm{mL}) \\
(\mathrm{Bq} / \mathrm{mL}) \\
(\mathrm{Bq} / \mathrm{mL}) \\
\\
(\mathrm{Bq} / \mathrm{mL}) \\
(\mathrm{Bq} / \mathrm{mL}) \\
(\mathrm{Bq} / \mathrm{mL}) \\
(\mathrm{Bq} / \mathrm{mL}) \\
(\mathrm{ng} / \mathrm{mL})\end{array}$ & $\begin{array}{l}270 \\
240 \\
4.4 \\
0.1 \\
2.4 \\
\\
6.1 \\
3.7 \\
2.4 \\
- \\
1.05 \\
136\end{array}$ & $\begin{array}{c}230 \\
280 \\
4.9 \\
0.1 \\
2.7 \\
\\
6.7 \\
4.0 \\
2.7 \\
- \\
1.18 \\
94\end{array}$ & $\begin{array}{c}240 \\
280 \\
4.9 \\
0.1 \\
2.6 \\
\\
8.4 \\
5.0 \\
3.4 \\
- \\
1.48 \\
65\end{array}$ & $\begin{array}{r}36 \\
22 \\
- \\
- \\
- \\
- \\
- \\
- \\
- \\
- \\
-\end{array}$ \\
\hline \multicolumn{6}{|c|}{ Uranium isotopics by TIMS $( \pm 0.5 \%)$} \\
\hline $\begin{array}{l}{ }^{233} \mathrm{U} \\
{ }^{234} \mathrm{U} \\
{ }^{236} \mathrm{U} U \\
{ }^{235} \mathrm{U} U{ }^{235} \mathrm{U} \text { FEM } \\
{ }^{233} \mathrm{U} \\
{ }^{233} \mathrm{U}\end{array}$ & $\begin{array}{l}\text { (atom \%) } \\
\text { (atom \%) } \\
\text { (atom \%) } \\
\text { (atom \%) } \\
\text { (atom \%) } \\
\\
(\mathrm{mg} / \mathrm{L}) \\
(\mathrm{mg} / \mathrm{L})\end{array}$ & $\begin{array}{r}0.35 \\
0.01 \\
0.53 \\
0.01 \\
99.10 \\
101 \\
\\
0.67 \\
1.0\end{array}$ & $\begin{array}{l}0.368 \\
0.010 \\
0.573 \\
0.009 \\
99.040 \\
\\
94 \\
\\
0.78 \\
1.2\end{array}$ & $\begin{array}{l}0.369 \\
0.010 \\
0.581 \\
0.009 \\
99.031 \\
\\
93 \\
\\
0.78 \\
1.2\end{array}$ & $\begin{array}{l}- \\
- \\
- \\
- \\
- \\
-\end{array}$ \\
\hline \multicolumn{6}{|c|}{ Uranium isotopics by ICP-MS ( $\pm 2 \%)$} \\
\hline $\begin{array}{l}{ }^{233} \mathrm{U} \\
{ }^{234} \mathrm{U} \\
{ }^{235} \mathrm{U} \\
{ }^{236} \mathrm{U} \\
{ }^{238} \mathrm{U} \\
{ }^{235} \mathrm{U} /{ }^{235} \mathrm{U} \text { FEM } \\
{ }^{233} \mathrm{U} \\
{ }^{235} \mathrm{U}\end{array}$ & $\begin{array}{l}\text { (atom \%) } \\
\text { (atom \%) } \\
\text { (atom \%) } \\
\text { (atom \%) } \\
\text { (atom \%) } \\
\\
(\mathrm{mg} / \mathrm{L}) \\
(\mathrm{mg} / \mathrm{L})\end{array}$ & $\begin{array}{l}- \\
- \\
- \\
- \\
- \\
-\end{array}$ & $\begin{array}{l}0.367 \\
0.010 \\
0.575 \\
0.009 \\
99.040 \\
\\
94 \\
\\
0.78 \\
1.2\end{array}$ & $\begin{array}{l}0.366 \\
0.010 \\
0.574 \\
0.008 \\
99.041 \\
\\
94 \\
\\
0.77 \\
1.2\end{array}$ & $\begin{array}{l}- \\
- \\
- \\
- \\
- \\
-\end{array}$ \\
\hline
\end{tabular}


Table 7 Analytical data for OHF tank T9 supernatant

\begin{tabular}{|c|c|c|c|c|c|}
\hline \multicolumn{2}{|l|}{$\begin{array}{l}\text { Characteristic } \\
\text { (Analysis) }\end{array}$} & $\begin{array}{c}\begin{array}{c}\text { T9-Center } \\
\text { liquid } \\
\text { (Winter 1996) }\end{array} \\
\end{array}$ & $\begin{array}{c}\text { T9-North } \\
\text { N9 liquid } \\
\text { (Winter 1997) }\end{array}$ & 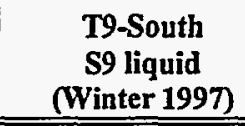 & $\begin{array}{c}\text { T9-L47 } \\
\text { liquid } \\
\text { (ER-13 1990) } \\
\end{array}$ \\
\hline \multicolumn{6}{|c|}{ Physical properties and miscellaneous data } \\
\hline $\begin{array}{l}\text { Request number } \\
\text { Sample number } \\
\text { pH } \\
\text { TSS }^{a} \\
\text { TDS }^{b} \\
\text { TS } \\
\text { Density } \\
\text { TIC }^{c} \\
\text { TC }^{d} \\
\text { TOC }^{c}\end{array}$ & $\begin{array}{l}(\mathrm{mg} / \mathrm{mL}) \\
(\mathrm{mg} / \mathrm{mL}) \\
(\mathrm{mg} / \mathrm{mL}) \\
(\mathrm{g} / \mathrm{mL}) \\
(\mathrm{mg} / \mathrm{L}) \\
(\mathrm{mg} / \mathrm{L}) \\
(\mathrm{mg} / \mathrm{L})\end{array}$ & $\begin{array}{c}7602 \mathrm{~B} \\
960123-153 \\
9.1 \\
0.08 \\
15.9 \\
16.1 \\
1.021 \\
413 \\
475 \\
62\end{array}$ & $\begin{array}{c}7907 \mathrm{~B} \\
961217-119 \\
8.9 \\
- \\
15.0 \\
- \\
1.021 \\
410 \\
470 \\
60\end{array}$ & $\begin{array}{c}7907 \mathrm{~B} \\
961217-118 \\
8.9 \\
- \\
14.0 \\
- \\
1.021 \\
400 \\
460 \\
60\end{array}$ & $\begin{array}{c}- \\
8.8 \\
0.7 \\
41.5 \\
42.2 \\
1.034 \\
- \\
850\end{array}$ \\
\hline \multicolumn{6}{|c|}{ RCRA Metals $( \pm 10 \%)$} \\
\hline $\begin{array}{ll}\mathrm{Ag} & (5)^{\mathrm{r}} \\
\mathrm{As} & (5) \\
\mathrm{Ba} & (100) \\
\mathrm{Cd} & (1) \\
\mathrm{Cr} & (5) \\
\mathrm{Hg} & (0.2) \\
\mathrm{Ni} & (50) \\
\mathrm{Pb} & (5) \\
\mathrm{Se} & (1) \\
\mathrm{Tl} & (0.9) \\
\end{array}$ & $\begin{array}{l}(\mathrm{mg} / \mathrm{L}) \\
(\mathrm{mg} / \mathrm{L}) \\
(\mathrm{mg} / \mathrm{L}) \\
(\mathrm{mg} / \mathrm{L}) \\
(\mathrm{mg} / \mathrm{L}) \\
(\mathrm{mg} / \mathrm{L}) \\
(\mathrm{mg} / \mathrm{L}) \\
(\mathrm{mg} / \mathrm{L}) \\
(\mathrm{mg} / \mathrm{L}) \\
(\mathrm{mg} / \mathrm{L})\end{array}$ & $\begin{array}{l}<0.02 \\
<0.008 \\
<0.002 \\
<0.04 \\
0.020 \\
0.896 \\
<0.04 \\
<0.008 \\
<0.008 \\
<0.008 \\
\end{array}$ & $\begin{array}{l}<0.03 \\
-0.007 \\
<0.03 \\
<0.008 \\
<-0.03 \\
- \\
-\end{array}$ & $\begin{array}{c}<0.03 \\
- \\
0.010 \\
<0.03 \\
0.0717 \\
<- \\
-0.03 \\
- \\
-\end{array}$ & $\begin{array}{c}0.01 \\
<0.8 \\
0.12 \\
<0.02 \\
0.4 \\
3.4 \\
<0.2 \\
<1 \\
<0.09 \\
<0.09 \\
\end{array}$ \\
\hline \multicolumn{6}{|c|}{ Process metals ( $\pm 10 \%$ ) } \\
\hline $\begin{array}{l}\mathrm{Al} \\
\mathrm{B} \\
\mathrm{Be} \\
\mathrm{Bi} \\
\mathrm{Ca} \\
\mathrm{Co} \\
\mathrm{Cu} \\
\mathrm{Cs} \\
\mathrm{Fe} \\
\mathrm{K} \\
\mathrm{Mg} \\
\mathrm{Mn} \\
\mathrm{Na} \\
\mathrm{P} \\
\mathrm{Sb} \\
\mathrm{Si} \\
\mathrm{Sr} \\
\mathrm{Th} \\
\mathrm{U} \\
\mathrm{V} \\
\mathrm{Zn}\end{array}$ & $\begin{array}{l}\text { (mg/L) } \\
(\mathrm{mg} / \mathrm{L}) \\
(\mathrm{mg} / \mathrm{L}) \\
(\mathrm{mg} / \mathrm{L}) \\
(\mathrm{mg} / \mathrm{L}) \\
(\mathrm{mg} / \mathrm{L}) \\
(\mathrm{mg} / \mathrm{L}) \\
(\mathrm{mg} / \mathrm{L}) \\
(\mathrm{mg} / \mathrm{L}) \\
(\mathrm{mg} / \mathrm{L}) \\
(\mathrm{mg} / \mathrm{L}) \\
(\mathrm{mg} / \mathrm{L}) \\
(\mathrm{mg} / \mathrm{L}) \\
(\mathrm{mg} / \mathrm{L}) \\
(\mathrm{mg} / \mathrm{L}) \\
(\mathrm{mg} / \mathrm{L}) \\
(\mathrm{mg} / \mathrm{L}) \\
(\mathrm{mg} / \mathrm{L}) \\
(\mathrm{mg} / \mathrm{L}) \\
(\mathrm{mg} / \mathrm{L}) \\
(\mathrm{mg} / \mathrm{L})\end{array}$ & $\begin{array}{c}0.249 \\
0.656 \\
<0.003 \\
- \\
14.2 \\
<0.02 \\
0.0902 \\
0.716 \\
<0.006 \\
695 \\
2.97 \\
<0.003 \\
4830 \\
25.6 \\
<0.37 \\
46.8 \\
1.02 \\
0.239 \\
303 \\
<0.007 \\
<0.049\end{array}$ & $\begin{array}{c}<0.06 \\
0.663 \\
<0.002 \\
5.77 \\
15.4 \\
0.050 \\
0.118 \\
- \\
<0.007 \\
591 \\
3.13 \\
0.0183 \\
4440 \\
3.27 \\
<0.33 \\
25.6 \\
0.917 \\
<0.083 \\
269 \\
<0.02 \\
<0.05\end{array}$ & $\begin{array}{c}<0.06 \\
0.672 \\
<0.002 \\
5.88 \\
15.3 \\
0.0533 \\
0.127 \\
- \\
<0.007 \\
600 \\
3.14 \\
0.020 \\
4490 \\
3.50 \\
<0.33 \\
101 \\
0.919 \\
<0.083 \\
275 \\
<0.02 \\
<0.05\end{array}$ & $\begin{array}{r}- \\
- \\
- \\
- \\
- \\
- \\
- \\
- \\
- \\
- \\
- \\
- \\
- \\
- \\
9.76 \\
- \\
- \\
852 \\
- \\
-\end{array}$ \\
\hline
\end{tabular}




\begin{tabular}{|c|c|c|c|c|c|}
\hline $\begin{array}{l}\text { Characteristic } \\
\text { (Analysis) }\end{array}$ & & $\begin{array}{c}\begin{array}{c}\text { T9-Center } \\
\text { liquid } \\
\text { (Winter 1996) }\end{array} \\
\end{array}$ & $\begin{array}{c}\text { T9-North } \\
\text { N9 liquid } \\
\text { (Winter 1997) } \\
\end{array}$ & $\begin{array}{c}\text { T9-South } \\
\text { S9 liquid } \\
\text { (Winter 1997) } \\
\end{array}$ & 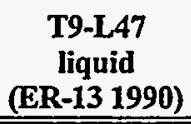 \\
\hline \multicolumn{6}{|c|}{ Semi-quantitative elements by ICP-MS ( $\pm 30-50 \%)$} \\
\hline $\begin{array}{l}\text { Cs } \\
\text { I } \\
\mathrm{Li} \\
\mathrm{Mo} \\
\mathrm{Rb} \\
\mathrm{Ru} \\
\mathrm{Sr} \\
\mathrm{Th} \\
\mathrm{Sn} \\
\mathrm{Ti} \\
\mathrm{W} \\
\mathrm{U}\end{array}$ & $\begin{array}{l}(\mathrm{mg} / \mathrm{L}) \\
(\mathrm{mg} / \mathrm{L}) \\
(\mathrm{mg} / \mathrm{L}) \\
(\mathrm{mg} / \mathrm{L}) \\
(\mathrm{mg} / \mathrm{L}) \\
(\mathrm{mg} / \mathrm{L}) \\
(\mathrm{mg} / \mathrm{L}) \\
(\mathrm{mg} / \mathrm{L}) \\
(\mathrm{mg} / \mathrm{L}) \\
(\mathrm{mg} / \mathrm{L}) \\
(\mathrm{mg} / \mathrm{L}) \\
(\mathrm{mg} / \mathrm{L})\end{array}$ & $\begin{array}{l}- \\
- \\
- \\
- \\
- \\
- \\
- \\
- \\
- \\
-\end{array}$ & $\begin{array}{l}0.33 \\
8.9 \\
4.7 \\
0.13 \\
0.17 \\
0.011 \\
1.1 \\
0.12 \\
0.020 \\
0.89 \\
0.016 \\
210\end{array}$ & $\begin{array}{l}0.34 \\
9.2 \\
4.5 \\
0.13 \\
0.20 \\
0.011 \\
0.99 \\
0.17 \\
<0.001 \\
0.95 \\
5.7 \\
200\end{array}$ & $\begin{array}{l}- \\
- \\
- \\
- \\
- \\
- \\
- \\
- \\
-\end{array}$ \\
\hline \multicolumn{6}{|c|}{ Anions by ion chromatography $( \pm 10 \%)$} \\
\hline $\begin{array}{l}\text { Inorganic } \\
\text { Bromide } \\
\text { Chloride } \\
\text { Chromate } \\
\text { Fluoride } \\
\text { Nitrite } \\
\text { Nitrate } \\
\text { Phosphate } \\
\text { Sulphate } \\
\text { Organic } \\
\text { Acetate } \\
\text { Citrate } \\
\text { Formate } \\
\text { Oxalate } \\
\text { Phthalate } \\
\text { Organic TICs } \\
\text { cis-Aconitate } \\
\text { trans-Aconitate } \\
\text { iso-Citrate } \\
\text { Malonate } \\
\text { Propionate }\end{array}$ & $\begin{array}{l}(\mathrm{mg} / \mathrm{L}) \\
(\mathrm{mg} / \mathrm{L}) \\
(\mathrm{mg} / \mathrm{L}) \\
(\mathrm{mg} / \mathrm{L}) \\
(\mathrm{mg} / \mathrm{L}) \\
(\mathrm{mg} / \mathrm{L}) \\
(\mathrm{mg} / \mathrm{L}) \\
(\mathrm{mg} / \mathrm{L}) \\
\\
(\mathrm{mg} / \mathrm{L}) \\
(\mathrm{mg} / \mathrm{L}) \\
(\mathrm{mg} / \mathrm{L}) \\
(\mathrm{mg} / \mathrm{L}) \\
(\mathrm{mg} / \mathrm{L}) \\
\\
(\mathrm{mg} / \mathrm{L}) \\
(\mathrm{mg} / \mathrm{L}) \\
(\mathrm{mg} / \mathrm{L}) \\
(\mathrm{mg} / \mathrm{L}) \\
(\mathrm{mg} / \mathrm{L})\end{array}$ & $\begin{array}{c}50.9 \\
5490 \\
- \\
19.5 \\
8.0 \\
2100 \\
<20 \\
821 \\
\\
- \\
- \\
- \\
- \\
- \\
- \\
- \\
- \\
-\end{array}$ & $\begin{array}{l}45.3 \\
4820 \\
<5 \\
14.2 \\
163 \\
1720 \\
<5 \\
666 \\
\\
<3 \\
<5 \\
<3 \\
<3 \\
<5 \\
\\
\\
\text { nd } \\
\text { nd } \\
\text { nd } \\
\text { nd } \\
\text { nd }\end{array}$ & $\begin{array}{l}44.6 \\
4810 \\
<5 \\
13.4 \\
152 \\
1640 \\
<5 \\
754 \\
\\
<3 \\
<5 \\
<3 \\
<3 \\
<5 \\
\\
\text { nd } \\
\text { nd } \\
\text { nd } \\
\text { nd } \\
\text { nd }\end{array}$ & $\begin{array}{c}290 \\
- \\
1.0 \\
- \\
110 \\
<5 \\
37\end{array}$ \\
\hline \multicolumn{6}{|c|}{ Beta/gamma emitters $( \pm 10 \%)$} \\
\hline $\begin{array}{l}\text { Gross beta } \\
{ }^{60} \mathrm{Co} \\
{ }^{134} \mathrm{Cs} \\
{ }^{137} \mathrm{Cs} /{ }^{137 m} \mathrm{Ba} \\
{ }^{152} \mathrm{Eu} \\
{ }^{154} \mathrm{Eu} \\
{ }^{155} \mathrm{Eu} \\
{ }^{90} \mathrm{Sr} /{ }^{90} \mathrm{Y} \\
{ }^{99} \mathrm{Tc}\end{array}$ & $\begin{array}{l}(\mathrm{Bq} / \mathrm{mL}) \\
(\mathrm{Bq} / \mathrm{mL}) \\
(\mathrm{Bq} / \mathrm{mL}) \\
(\mathrm{Bq} / \mathrm{mL}) \\
(\mathrm{Bq} / \mathrm{mL}) \\
(\mathrm{Bq} / \mathrm{mL}) \\
(\mathrm{Bq} / \mathrm{mL}) \\
(\mathrm{Bq} / \mathrm{mL}) \\
(\mathrm{Bq} / \mathrm{mL})\end{array}$ & $\begin{array}{r}120000 \\
28 \\
<59 \\
92000 \\
<11 \\
<35 \\
<170 \\
10000 \\
9.0\end{array}$ & $\begin{array}{r}120000 \\
<120 \\
<190 \\
84000 \\
<110 \\
<190 \\
<520 \\
7700 \\
-\end{array}$ & $\begin{array}{r}120000 \\
<21 \\
<190 \\
84000 \\
<410 \\
<200 \\
<520 \\
8300 \\
-\end{array}$ & $\begin{array}{c}340000 \\
0.6 \\
- \\
290000 \\
- \\
- \\
- \\
- \\
-\end{array}$ \\
\hline
\end{tabular}




\begin{tabular}{|c|c|c|c|c|c|}
\hline $\begin{array}{l}\text { Characteristic } \\
\text { (Analysis) }\end{array}$ & & $\begin{array}{c}\begin{array}{c}\text { T9-Center } \\
\text { liquid } \\
\text { (Winter 1996) }\end{array} \\
\end{array}$ & $\begin{array}{c}\text { T9-North } \\
\text { N9 liquid } \\
\text { (Winter 1997) } \\
\end{array}$ & $\begin{array}{c}\text { T9-South } \\
\text { S9 liquid } \\
\text { (Winter 1997) }\end{array}$ & $\begin{array}{c}\text { T9-L47 } \\
\text { liquid } \\
\text { (ER-13 1990) } \\
\end{array}$ \\
\hline \multicolumn{6}{|c|}{ Alpha emitters $( \pm 10 \%)$} \\
\hline $\begin{array}{l}\text { Gross alpha } \\
{ }^{233} \mathrm{U} \\
{ }^{234} \mathrm{U} \\
{ }^{233} \mathrm{U} \\
{ }^{238} \mathrm{U} \\
\text { Total Pu alpha } \\
{ }^{238} \mathrm{Pu} \\
{ }^{239} \mathrm{Pu} /{ }^{40} \mathrm{Pu} \\
{ }^{242} \mathrm{Pu} \\
\left.{ }^{[232} \mathrm{Pu}\right]\end{array}$ & $\begin{array}{l}(\mathrm{Bq} / \mathrm{mL}) \\
(\mathrm{Bq} / \mathrm{mL}) \\
(\mathrm{Bq} / \mathrm{mL}) \\
(\mathrm{Bq} / \mathrm{mL}) \\
(\mathrm{Bq} / \mathrm{mL}) \\
\\
(\mathrm{Bq} / \mathrm{mL}) \\
(\mathrm{Bq} / \mathrm{mL}) \\
(\mathrm{Bq} / \mathrm{mL}) \\
(\mathrm{Bq} / \mathrm{mL}) \\
(\mathrm{ng} / \mathrm{mL})\end{array}$ & $\begin{array}{c}500 \\
470 \\
6.9 \\
0.1 \\
3.7 \\
\\
1.3 \\
0.88 \\
0.42 \\
- \\
0.18 \\
1310\end{array}$ & $\begin{array}{c}190 \\
420 \\
6.7 \\
0.1 \\
3.3 \\
\\
0.49 \\
0.43 \\
0.06 \\
- \\
0.24 \\
501\end{array}$ & $\begin{array}{c}300 \\
430 \\
6.2 \\
0.1 \\
3.4 \\
\\
0.56 \\
0.46 \\
0.10 \\
- \\
0.04 \\
3900\end{array}$ & $\begin{array}{r}700 \\
660 \\
- \\
- \\
- \\
- \\
- \\
- \\
- \\
- \\
-\end{array}$ \\
\hline \multicolumn{6}{|c|}{ Uranium isotopics by TIMS $( \pm 0.5 \%)$} \\
\hline $\begin{array}{l}{ }^{233} U \\
{ }^{234} U \\
{ }^{235} U \\
{ }^{236} U \\
{ }^{238} U \\
{ }^{238} U / 235 U \text { FEM } \\
{ }^{233} \mathrm{U} \\
{ }^{235} \mathrm{U}\end{array}$ & $\begin{array}{l}\text { (atom \%) } \\
\text { (atom \%) } \\
\text { (atom \%) } \\
\text { (atom \%) } \\
\text { (atom \%) } \\
\\
(\mathrm{mg} / \mathrm{L}) \\
(\mathrm{mg} / \mathrm{L})\end{array}$ & $\begin{array}{r}0.44 \\
0.01 \\
0.52 \\
0.01 \\
99.02 \\
\\
90 \\
\\
1.3 \\
1.6\end{array}$ & $\begin{array}{r}0.446 \\
0.011 \\
0.546 \\
0.009 \\
98.988 \\
88 \\
\\
1.2 \\
1.5\end{array}$ & $\begin{array}{r}0.447 \\
0.010 \\
0.551 \\
0.009 \\
98.983 \\
87 \\
\\
1.2 \\
1.5\end{array}$ & $\begin{array}{l}- \\
- \\
- \\
- \\
- \\
- \\
-\end{array}$ \\
\hline \multicolumn{6}{|c|}{ Uranium isotopics by ICP-MS ( $\pm 2 \%)$} \\
\hline $\begin{array}{l}{ }^{233} \mathrm{U} \\
{ }^{234} \mathrm{U} \\
{ }^{235} \mathrm{U} \\
{ }^{236} \mathrm{U} \\
{ }^{238} \mathrm{U} \\
{ }^{238} \mathrm{U} /{ }^{235} \mathrm{U} \text { FEM } \\
{ }^{233} \mathrm{U} \\
{ }^{235} \mathrm{U}\end{array}$ & $\begin{array}{l}\text { (atom \%) } \\
\text { (atom \%) } \\
\text { (atom \%) } \\
\text { (atom \%) } \\
\text { (atom \%) } \\
\\
(\mathrm{mg} / \mathrm{L}) \\
(\mathrm{mg} / \mathrm{L})\end{array}$ & $\begin{array}{l}- \\
- \\
- \\
- \\
- \\
-\end{array}$ & $\begin{array}{r}0.444 \\
0.012 \\
0.538 \\
0.008 \\
98.998 \\
\\
89 \\
\\
1.2 \\
1.4\end{array}$ & $\begin{array}{r}0.440 \\
0.011 \\
0.537 \\
0.010 \\
99.003 \\
\\
89 \\
\\
1.2 \\
1.5\end{array}$ & $\begin{array}{l}- \\
- \\
- \\
- \\
- \\
-\end{array}$ \\
\hline
\end{tabular}


Table 8 Analytical data for OHF tank T1 sludge

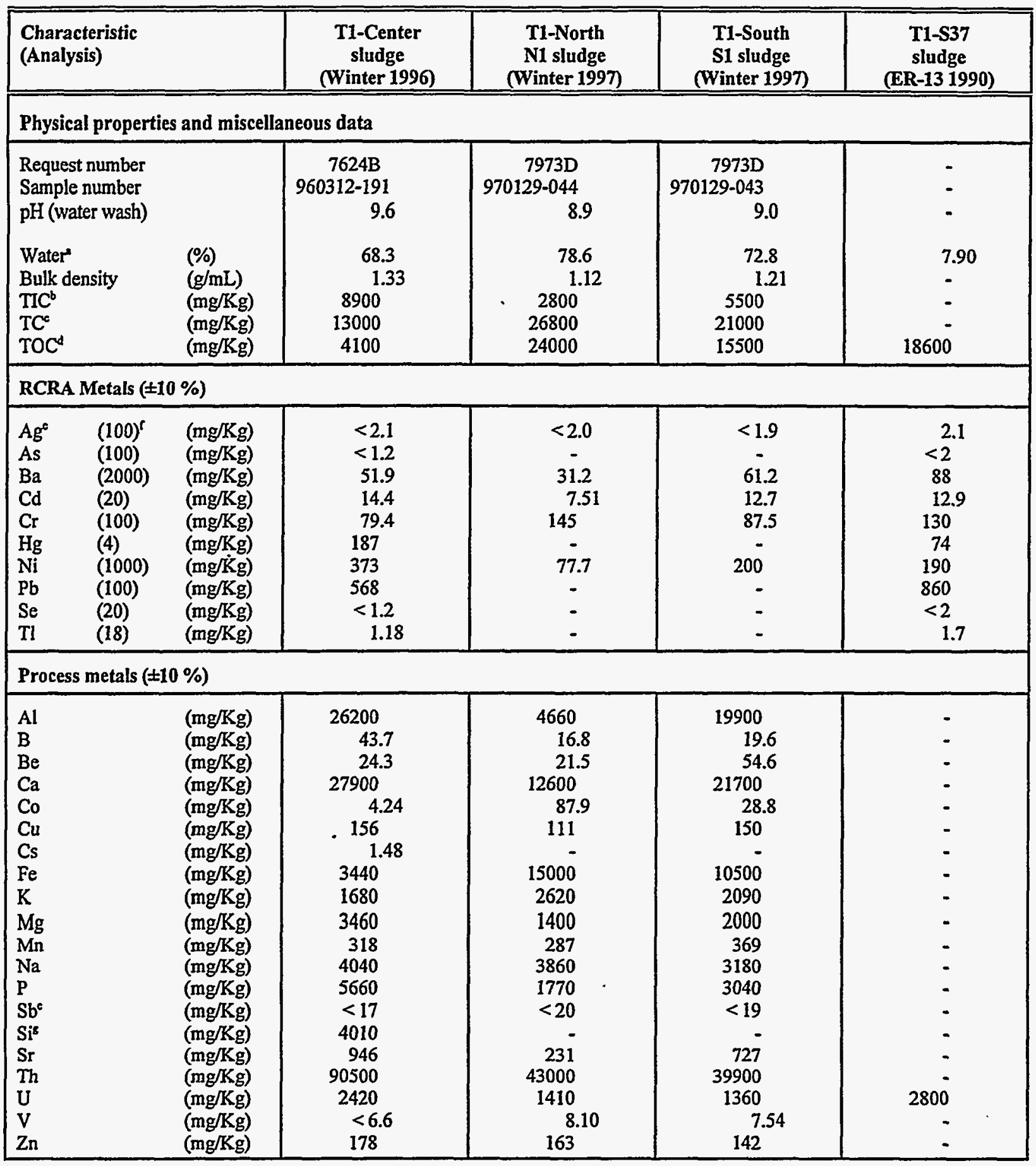




\begin{tabular}{|c|c|c|c|c|c|}
\hline $\begin{array}{l}\text { Characteristic } \\
\text { (Analysis) }\end{array}$ & & $\begin{array}{c}\begin{array}{c}\text { T1-Center } \\
\text { sludge } \\
\text { (Winter 1996) }\end{array} \\
\end{array}$ & $\begin{array}{c}\text { T1-North } \\
\text { N1 sludge } \\
\text { (Winter 1997) } \\
\end{array}$ & $\begin{array}{c}\text { T1-South } \\
\text { S1 sludge } \\
\text { (Winter 1997) } \\
\end{array}$ & 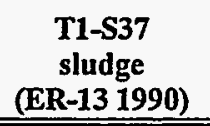 \\
\hline \multicolumn{6}{|c|}{ Semi-quantitative elements by ICP-MS ( $\pm 30-50 \%)$} \\
\hline $\begin{array}{l}\mathrm{Bi} \\
\mathrm{Ga} \\
\mathrm{I} \\
\mathrm{Li} \\
\mathrm{Te} \\
\mathrm{Zr} \\
\text { Lanthanides } \\
\text { (57) } \mathrm{La} \\
(58) \mathrm{Ce} \\
(59) \mathrm{Pr} \\
(60) \mathrm{Nd} \\
(62) \mathrm{Sm} \\
(63) \mathrm{Eu}\end{array}$ & $\begin{array}{l}(\mathrm{mg} / \mathrm{Kg}) \\
(\mathrm{mg} / \mathrm{Kg}) \\
(\mathrm{mg} / \mathrm{Kg}) \\
(\mathrm{mg} / \mathrm{Kg}) \\
(\mathrm{mg} / \mathrm{Kg}) \\
(\mathrm{mg} / \mathrm{Kg}) \\
\\
(\mathrm{mg} / \mathrm{Kg}) \\
(\mathrm{mg} / \mathrm{Kg}) \\
(\mathrm{mg} / \mathrm{Kg}) \\
(\mathrm{mg} / \mathrm{Kg}) \\
(\mathrm{mg} / \mathrm{Kg}) \\
(\mathrm{mg} / \mathrm{Kg})\end{array}$ & $\begin{array}{l}- \\
: \\
- \\
- \\
- \\
- \\
- \\
-\end{array}$ & $\begin{array}{l}12 \\
2.4 \\
41 \\
410 \\
63 \\
21 \\
\\
7.0 \\
7.8 \\
0.91 \\
5.5 \\
0.97 \\
0.54\end{array}$ & $\begin{array}{c}46 \\
6.6 \\
24 \\
1380 \\
42 \\
39 \\
\\
10 \\
12 \\
2.4 \\
8.4 \\
5.7 \\
0.48\end{array}$ & $\begin{array}{l}- \\
- \\
- \\
- \\
- \\
- \\
-\end{array}$ \\
\hline \multicolumn{6}{|c|}{ Anions on water wash by ion chromatography ( $\pm 10 \%$ ) } \\
\hline $\begin{array}{l}\text { Inorganic } \\
\text { Bromide } \\
\text { Chloride } \\
\text { Chromate } \\
\text { Fluoride } \\
\text { Nitrate } \\
\text { Nitrite } \\
\text { Phosphate } \\
\text { Sulphate } \\
\text { Organic } \\
\text { Acetate } \\
\text { Citrate } \\
\text { Formate } \\
\text { Oxalate } \\
\text { Phthalate } \\
\text { Organic TICs } \\
\text { cis-Aconitate } \\
\text { trans-Aconitate } \\
\text { iso-Citrate } \\
\text { Malonate } \\
\text { Propionate } \\
\end{array}$ & $\begin{array}{l}(\mathrm{mg} / \mathrm{Kg}) \\
(\mathrm{mg} / \mathrm{Kg}) \\
(\mathrm{mg} / \mathrm{Kg}) \\
(\mathrm{mg} / \mathrm{Kg}) \\
(\mathrm{mg} / \mathrm{Kg}) \\
(\mathrm{mg} / \mathrm{Kg}) \\
(\mathrm{mg} / \mathrm{Kg}) \\
(\mathrm{mg} / \mathrm{Kg}) \\
\\
(\mathrm{mg} / \mathrm{Kg}) \\
(\mathrm{mg} / \mathrm{Kg}) \\
(\mathrm{mg} / \mathrm{Kg}) \\
(\mathrm{mg} / \mathrm{Kg}) \\
(\mathrm{mg} / \mathrm{Kg}) \\
\\
(\mathrm{mg} / \mathrm{Kg}) \\
(\mathrm{mg} / \mathrm{Kg}) \\
(\mathrm{mg} / \mathrm{Kg}) \\
(\mathrm{mg} / \mathrm{Kg}) \\
(\mathrm{mg} / \mathrm{Kg}) \\
\end{array}$ & $\begin{array}{c}<4.6 \\
247 \\
- \\
176 \\
52.6 \\
629 \\
<19 \\
339\end{array}$ & $\begin{array}{l}<4.8 \\
283 \\
<10 \\
181 \\
29.6 \\
1030 \\
<10 \\
348 \\
\\
\\
<5 \\
<10 \\
<5 \\
<5 \\
<10 \\
\\
\\
\text { nd } \\
\text { nd } \\
\text { nd } \\
\text { nd } \\
\text { nd } \\
\end{array}$ & $\begin{array}{l}<4.8 \\
296 \\
<10 \\
117 \\
46.7 \\
1080 \\
<10 \\
365 \\
\\
\\
<5 \\
<10 \\
8.26 \\
303 \\
<10 \\
\\
\\
\\
\text { nd } \\
\text { nd } \\
\text { nd } \\
\text { nd } \\
\text { nd } \\
\end{array}$ & $\begin{array}{l}- \\
- \\
- \\
- \\
- \\
- \\
-\end{array}$ \\
\hline \multicolumn{6}{|c|}{ Beta/gamma emitters $( \pm 10 \%)$} \\
\hline $\begin{array}{l}\frac{\text { Gross beta }}{{ }_{114} \mathrm{C}} \\
{ }^{60} \mathrm{Co} \\
{ }^{90} \mathrm{Sr} \mathrm{Pr}^{80} \mathrm{Y} \\
{ }^{99} \mathrm{Tc} \\
{ }^{134} \mathrm{Cs} \\
{ }^{137} \mathrm{Cs} / s^{137 m} \mathrm{Ba} \\
{ }^{152} \mathrm{Eu} \\
{ }^{154} \mathrm{Eu} \\
{ }^{155} \mathrm{Eu} \\
{ }^{241} \mathrm{Pu}\end{array}$ & $\begin{array}{l}(\mathrm{Bq} / \mathrm{g}) \\
(\mathrm{Bq} / \mathrm{g}) \\
(\mathrm{Bq} / \mathrm{g}) \\
(\mathrm{Bq} / \mathrm{g}) \\
(\mathrm{Bq} / \mathrm{g}) \\
(\mathrm{Bq} / \mathrm{g}) \\
(\mathrm{Bq} / \mathrm{g}) \\
(\mathrm{Bq} / \mathrm{g}) \\
(\mathrm{Bq} / \mathrm{g}) \\
(\mathrm{Bq} / \mathrm{g}) \\
(\mathrm{Bq} / \mathrm{g})\end{array}$ & $\begin{array}{r}4.5 e+07 \\
- \\
6.7 e+04 \\
2.0 e+07 \\
1.3 e+01 \\
<4.9 e+02 \\
3.9 e+05 \\
6.3 e+04 \\
4.3 e+04 \\
<2.7 e+03 \\
2.9 e+04\end{array}$ & $\begin{array}{r}1.8 \mathrm{e}+07 \\
- \\
4.9 \mathrm{e}+04 \\
6.6 \mathrm{e}+06 \\
- \\
<1.5 \mathrm{e}+03 \\
4.5 \mathrm{e}+05 \\
4.3 \mathrm{e}+04 \\
3.3 \mathrm{e}+04 \\
<6.2 \mathrm{e}+03 \\
-\end{array}$ & $\begin{array}{l}3.9 \mathrm{e}+07 \\
- \\
2.4 \mathrm{e}+04 \\
1.3 \mathrm{e}+07 \\
- \\
<1.4 \mathrm{e}+03 \\
2.9 \mathrm{e}+05 \\
2.3 \mathrm{e}+04 \\
2.0 \mathrm{e}+04 \\
<7.7 \mathrm{e}+03 \\
-\end{array}$ & $\begin{array}{l}5.9 e+07 \\
4.8 e+01 \\
2.6 e+05 \\
3.2 e+07 \\
- \\
- \\
3.9 e+05 \\
1.4 e+05 \\
1.2 e+05 \\
2.3 e+04 \\
-\end{array}$ \\
\hline
\end{tabular}




\begin{tabular}{|c|c|c|c|c|c|}
\hline \multicolumn{2}{|l|}{$\begin{array}{l}\text { Characteristic } \\
\text { (Analysis) }\end{array}$} & $\begin{array}{c}\begin{array}{c}\text { T1-Center } \\
\text { sludge } \\
\text { (Winter 1996) }\end{array} \\
\end{array}$ & $\begin{array}{c}\text { T1-North } \\
\text { N1 sludge } \\
\text { (Winter 1997) } \\
\end{array}$ & $\begin{array}{c}\text { T1-South } \\
\text { S1 sludge } \\
\text { (Winter 1997) } \\
\end{array}$ & $\begin{array}{c}\text { T1-S37 } \\
\text { sludge } \\
\text { (ER-13 1990) } \\
\end{array}$ \\
\hline \multicolumn{6}{|c|}{ Alpha emitters $( \pm 10 \%)$} \\
\hline $\begin{array}{l}\text { Gross alpha } \\
{ }^{232} \mathrm{Th} \\
{ }^{233} \mathrm{U} \\
{ }^{234} \mathrm{U} \\
{ }^{233} \mathrm{U} \\
{ }^{238} \mathrm{U} \\
{ }^{237} \mathrm{~Np} \\
{ }^{241} \mathrm{Am} \\
{ }^{244} \mathrm{Cm} \\
{ }^{252} \mathrm{Cf} \\
\text { Total Pu alpha } \\
{ }^{238} \mathrm{Pu} \\
{ }^{239} \mathrm{Pu} /{ }^{240} \mathrm{Pu} \\
{ }^{212} \mathrm{Pu}\end{array}$ & $\begin{array}{l}(\mathrm{Bq} / \mathrm{g}) \\
(\mathrm{Bq} / \mathrm{g}) \\
(\mathrm{Bq} / \mathrm{g}) \\
(\mathrm{Bq} / \mathrm{g}) \\
(\mathrm{Bq} / \mathrm{g}) \\
(\mathrm{Bq} / \mathrm{g}) \\
(\mathrm{Bq} / \mathrm{g}) \\
(\mathrm{Bq} / \mathrm{g}) \\
(\mathrm{Bq} / \mathrm{g}) \\
(\mathrm{Bq} / \mathrm{g}) \\
\vdots \\
(\mathrm{Bq} / \mathrm{g}) \\
(\mathrm{Bq} / \mathrm{g}) \\
(\mathrm{Bq} / \mathrm{g}) \\
(\mathrm{Bq} / \mathrm{g})\end{array}$ & $\begin{array}{l}4.6 e+05 \\
3.7 e+02 \\
7.9 e+03 \\
1.1 e+02 \\
0.7 e+00 \\
3.0 e+01 \\
9.0 e+00 \\
5.2 e+04 \\
3.5 e+05 \\
<4.6 e+02 \\
4.0 e+04 \\
2.9 e+04 \\
1.1 e+04 \\
-\end{array}$ & $\begin{array}{l}2.6 e+05 \\
1.8 e+02 \\
6.1 e+03 \\
6.1 e+01 \\
0.4 e+00 \\
1.7 e+01 \\
- \\
1.1 e+04 \\
2.3 e+05 \\
- \\
1.5 e+04 \\
9.7 e+03 \\
4.9 e+03 \\
-\end{array}$ & $\begin{array}{l}2.5 e+05 \\
1.6 e+02 \\
3.0 e+03 \\
4.0 e+01 \\
0.5 e+00 \\
1.7 e+01 \\
- \\
1.7 e+04 \\
2.0 e+05 \\
- \\
2.1 e+04 \\
1.6 e+04 \\
5.8 e+03 \\
-\end{array}$ & $\begin{array}{l}6.5 e+05 \\
- \\
- \\
- \\
- \\
- \\
- \\
- \\
- \\
- \\
- \\
3.4 e+04 \\
6.5 e+03 \\
-\end{array}$ \\
\hline \multicolumn{6}{|c|}{ Uranium isotopics by TIMS ( $\neq 0.5 \%)$} \\
\hline $\begin{array}{l}{ }^{233} U \\
{ }^{234} \mathrm{U} \\
{ }^{235} \mathrm{U} \\
{ }^{236} \mathrm{U} \\
{ }^{238} \mathrm{U} /{ }^{235} \mathrm{U} \mathrm{FEM}^{\mathrm{h}} \\
{ }^{233} \mathrm{U} \\
{ }^{235} \mathrm{U}\end{array}$ & $\begin{array}{l}\text { (atom \%) } \\
\text { (atom \%) } \\
\text { (atom \%) } \\
\text { (atom \%) } \\
\text { (atom \%) } \\
\\
(\mathrm{mg} / \mathrm{Kg}) \\
(\mathrm{mg} / \mathrm{Kg})\end{array}$ & $\begin{array}{r}0.93 \\
0.02 \\
0.43 \\
0.01 \\
98.61 \\
\\
60 \\
\\
22 \\
10\end{array}$ & $\begin{array}{c}1.234 \\
0.019 \\
0.378 \\
0.006 \\
98.363 \\
49 \\
17 \\
5.3\end{array}$ & $\begin{array}{r}0.634 \\
0.013 \\
0.506 \\
0.008 \\
98.839 \\
74 \\
\\
8.4 \\
6.8\end{array}$ & $\begin{array}{l}- \\
- \\
- \\
- \\
- \\
-\end{array}$ \\
\hline \multicolumn{6}{|c|}{ Uranium isotopics by ICP-MS ( $\pm 2 \%)$} \\
\hline $\begin{array}{l}{ }^{233} \mathrm{U} \\
{ }^{234} \mathrm{U} \\
{ }^{235} \mathrm{U} \\
{ }^{236} \mathrm{U} \\
{ }^{238} \mathrm{U} /{ }^{235} \mathrm{U} \mathrm{FEM}^{\mathrm{h}} \\
{ }^{233} \mathrm{U} \\
{ }^{235} \mathrm{U}\end{array}$ & $\begin{array}{l}\text { (atom \%) } \\
\text { (atom \%) } \\
\text { (atom \%) } \\
\text { (atom \%) } \\
\text { (atom \%) } \\
\\
(\mathrm{mg} / \mathrm{Kg}) \\
(\mathrm{mg} / \mathrm{Kg})\end{array}$ & $\begin{array}{l}- \\
- \\
- \\
- \\
- \\
- \\
-\end{array}$ & $\begin{array}{c}1.216 \\
0.020 \\
0.378 \\
0.008 \\
98.378 \\
50 \\
\\
15 \\
4.8\end{array}$ & $\begin{array}{l}0.632 \\
0.014 \\
0.504 \\
0.007 \\
98.843 \\
74 \\
\\
8.1 \\
6.5\end{array}$ & $\begin{array}{l}- \\
- \\
- \\
- \\
- \\
- \\
-\end{array}$ \\
\hline
\end{tabular}




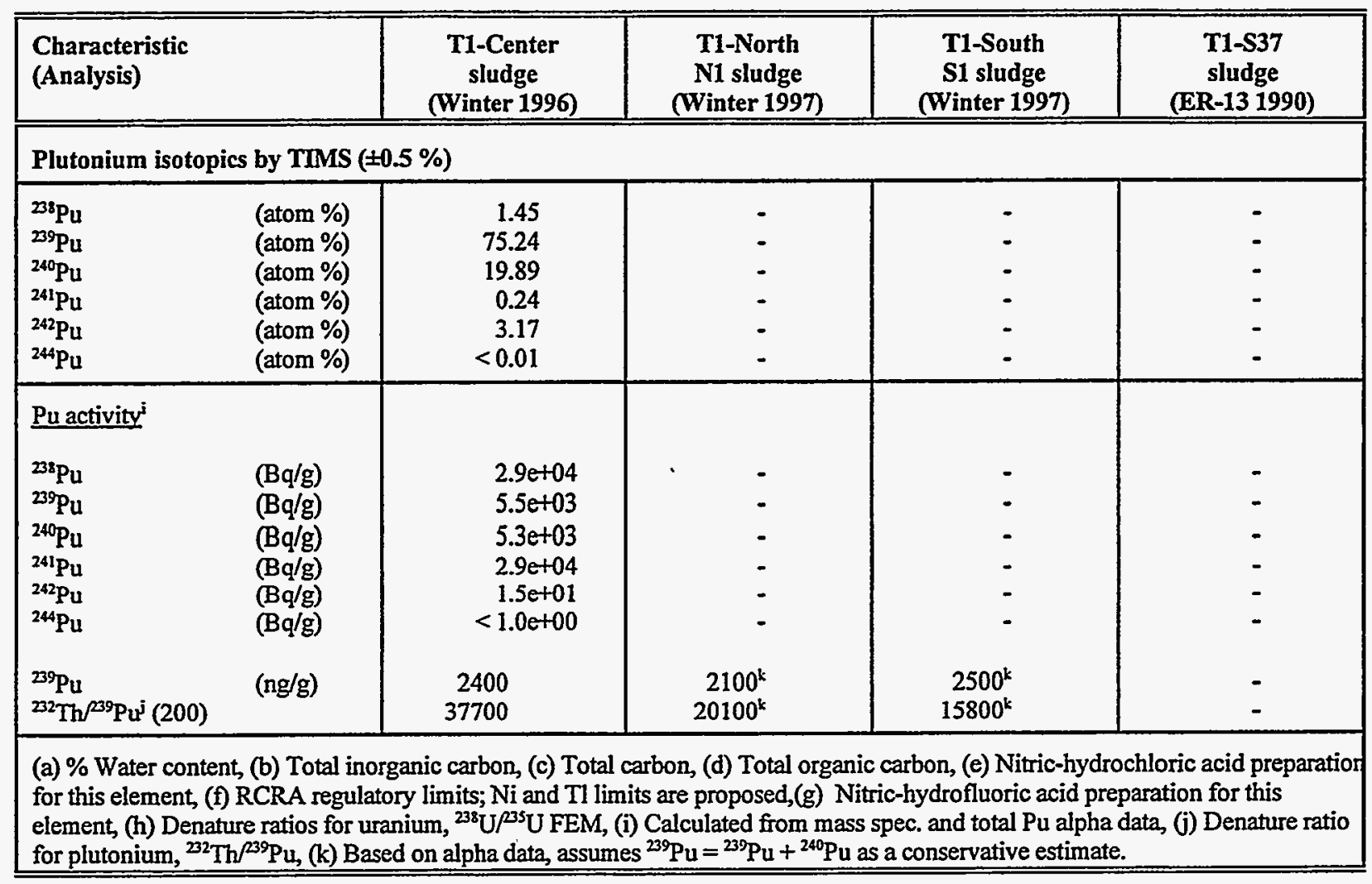


Table 9 Analytical data for OHF tank T2 sludge

\begin{tabular}{|c|c|c|c|c|c|c|}
\hline \multicolumn{3}{|c|}{$\begin{array}{l}\text { Characteristic } \\
\text { (Analysis) }\end{array}$} & $\begin{array}{c}\begin{array}{c}\text { T2-Center } \\
\text { sludge } \\
\text { (Winter 1996) }\end{array} \\
\end{array}$ & $\begin{array}{c}\text { T2-North } \\
\text { N2 sludge } \\
\text { (Winter 1997) } \\
\end{array}$ & 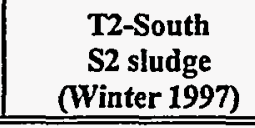 & 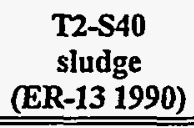 \\
\hline \multicolumn{7}{|c|}{ Physical properties and miscellaneous data } \\
\hline \multicolumn{3}{|c|}{$\begin{array}{l}\text { Request number } \\
\text { Sample number } \\
\text { pH (water wash) }\end{array}$} & $\begin{array}{r}7624 \mathrm{~A} \\
960306-120 \\
9.6\end{array}$ & $\begin{array}{r}7973 \mathrm{E} \\
970129-046 \\
9.2\end{array}$ & $\begin{array}{r}\text { 7973E } \\
970129-045 \\
9.3\end{array}$ & - \\
\hline \multicolumn{2}{|c|}{$\begin{array}{l}\text { Water } \\
\text { Bulk density } \\
\text { TIC } \\
\text { TC }^{\mathbf{c}} \\
\text { TOC }^{d}\end{array}$} & $\begin{array}{l}(\%) \\
(\mathrm{g} / \mathrm{mL}) \\
(\mathrm{mg} / \mathrm{Kg}) \\
(\mathrm{mg} / \mathrm{Kg}) \\
(\mathrm{mg} / \mathrm{Kg})\end{array}$ & $\begin{array}{c}61.9 \\
1.33 \\
16000 \\
29000 \\
13000\end{array}$ & $\begin{array}{c}78.8 \\
1.18 \\
4200 \\
18600 \\
14400\end{array}$ & $\begin{array}{c}76.9 \\
1.16 \\
4600 \\
10300 \\
5700\end{array}$ & $\begin{array}{c}67.6 \\
- \\
- \\
28000\end{array}$ \\
\hline \multicolumn{7}{|c|}{ RCRA Metals ( $\pm 10 \%)$} \\
\hline & $\begin{array}{l}(100)^{r} \\
(100) \\
(2000) \\
(20) \\
(100) \\
(4) \\
(1000) \\
(100) \\
(20) \\
(18)\end{array}$ & $\begin{array}{l}(\mathrm{mg} / \mathrm{Kg}) \\
(\mathrm{mg} / \mathrm{Kg}) \\
(\mathrm{mg} / \mathrm{Kg}) \\
(\mathrm{mg} / \mathrm{Kg}) \\
(\mathrm{mg} / \mathrm{Kg}) \\
(\mathrm{mg} / \mathrm{Kg}) \\
(\mathrm{mg} / \mathrm{Kg}) \\
(\mathrm{mg} / \mathrm{Kg}) \\
(\mathrm{mg} / \mathrm{Kg}) \\
(\mathrm{mg} / \mathrm{Kg})\end{array}$ & $\begin{array}{c}<1.0 \\
<1.2 \\
52.3 \\
14.4 \\
241 \\
196 \\
173 \\
654 \\
<1.2 \\
1.4\end{array}$ & $\begin{array}{c}<2.0 \\
- \\
43.2 \\
10.8 \\
323 \\
- \\
529 \\
- \\
\therefore-\end{array}$ & $\begin{array}{c}<1.7 \\
- \\
37.0 \\
12.0 \\
87.1 \\
- \\
176 \\
- \\
- \\
-\end{array}$ & $\begin{array}{c}2.9 \\
<1 \\
33 \\
6.6 \\
180 \\
70 \\
72 \\
350 \\
<1 \\
<1 \\
\end{array}$ \\
\hline \multicolumn{7}{|c|}{ Process metals ( $\pm 10 \%)$} \\
\hline $\begin{array}{l}\mathrm{Al} \\
\mathrm{B} \\
\mathrm{Be} \\
\mathrm{Ca} \\
\mathrm{Co} \\
\mathrm{Cu} \\
\mathrm{Cs} \\
\mathrm{Fe} \\
\mathrm{K} \\
\mathrm{Mg} \\
\mathrm{Mn} \\
\mathrm{Na} \\
\mathrm{P} \\
\mathrm{Sb}^{\mathrm{c}} \\
\mathrm{Si}^{8} \\
\mathrm{Sr} \\
\mathrm{Th} \\
\mathrm{U} \\
\mathrm{V} \\
\mathrm{Zn}\end{array}$ & & $\begin{array}{l}(\mathrm{mg} / \mathrm{Kg}) \\
(\mathrm{mg} / \mathrm{Kg}) \\
(\mathrm{mg} / \mathrm{Kg}) \\
(\mathrm{mg} / \mathrm{Kg}) \\
(\mathrm{mg} / \mathrm{Kg}) \\
(\mathrm{mg} / \mathrm{Kg}) \\
(\mathrm{mg} / \mathrm{Kg}) \\
(\mathrm{mg} / \mathrm{Kg}) \\
(\mathrm{mg} / \mathrm{Kg}) \\
(\mathrm{mg} / \mathrm{Kg}) \\
(\mathrm{mg} / \mathrm{Kg}) \\
(\mathrm{mg} / \mathrm{Kg}) \\
(\mathrm{mg} / \mathrm{Kg}) \\
(\mathrm{mg} / \mathrm{Kg}) \\
(\mathrm{mg} / \mathrm{Kg}) \\
(\mathrm{mg} / \mathrm{Kg}) \\
(\mathrm{mg} / \mathrm{Kg}) \\
(\mathrm{mg} / \mathrm{Kg}) \\
(\mathrm{mg} / \mathrm{Kg}) \\
(\mathrm{mg} / \mathrm{Kg})\end{array}$ & $\begin{array}{c}15900 \\
43.9 \\
19.6 \\
36600 \\
14.2 \\
126 \\
22.5 \\
6240 \\
2130 \\
3170 \\
336 \\
5060 \\
6200 \\
<17 \\
3950 \\
992 \\
94300 \\
2090 \\
<6.7 \\
236 \\
\end{array}$ & $\begin{array}{c}9390 \\
36.2 \\
23.9 \\
12900 \\
304 \\
262 \\
- \\
48900 \\
2270 \\
2580 \\
555 \\
3870 \\
1750 \\
<20 \\
- \\
261 \\
24200 \\
832 \\
5.88 \\
110 \\
\end{array}$ & $\begin{array}{c}20300 \\
22.1 \\
59.1 \\
12900 \\
15.1 \\
93.5 \\
- \\
2940 \\
2490 \\
1410 \\
264 \\
4500 \\
2100 \\
<17 \\
- \\
460 \\
54800 \\
1350 \\
5.26 \\
129 \\
\end{array}$ & $\begin{array}{r}- \\
- \\
- \\
- \\
- \\
- \\
- \\
- \\
- \\
- \\
- \\
- \\
- \\
- \\
-\end{array}$ \\
\hline
\end{tabular}




\begin{tabular}{|c|c|c|c|c|c|}
\hline $\begin{array}{l}\text { Characteristic } \\
\text { (Analysis) }\end{array}$ & & $\begin{array}{c}\begin{array}{c}\text { T2-Center } \\
\text { sludge } \\
\text { (Winter 1996) }\end{array} \\
\end{array}$ & $\begin{array}{c}\text { T2-North } \\
\text { N2 sludge } \\
\text { (Winter 1997) } \\
\end{array}$ & $\begin{array}{c}\text { T2-South } \\
\text { S2 sludge } \\
\text { (Winter 1997) }\end{array}$ & $\begin{array}{c}\text { T2-S40 } \\
\text { sludge } \\
\text { (ER-13 1990) }\end{array}$ \\
\hline \multicolumn{6}{|c|}{ Semi-quantitative elements by ICP-MS ( $\pm 30-50 \%)$} \\
\hline $\begin{array}{l}\mathrm{Bi} \\
\mathrm{Ga} \\
\mathrm{I} \\
\mathrm{Li} \\
\mathrm{Te} \\
\mathrm{Zr} \\
\text { Lanthanides } \\
\text { (57) } \mathrm{La} \\
(58) \mathrm{Ce} \\
(59) \mathrm{Pr} \\
(60) \mathrm{Nd} \\
(62) \mathrm{Sm} \\
(63) \mathrm{Eu}\end{array}$ & $\begin{array}{l}(\mathrm{mg} / \mathrm{Kg}) \\
(\mathrm{mg} / \mathrm{Kg}) \\
(\mathrm{mg} / \mathrm{Kg}) \\
(\mathrm{mg} / \mathrm{Kg}) \\
(\mathrm{mg} / \mathrm{Kg}) \\
(\mathrm{mg} / \mathrm{Kg}) \\
\\
(\mathrm{mg} / \mathrm{Kg}) \\
(\mathrm{mg} / \mathrm{Kg}) \\
(\mathrm{mg} / \mathrm{Kg}) \\
(\mathrm{mg} / \mathrm{Kg}) \\
(\mathrm{mg} / \mathrm{Kg}) \\
(\mathrm{mg} / \mathrm{Kg})\end{array}$ & $\begin{array}{l}- \\
- \\
- \\
- \\
- \\
- \\
- \\
- \\
-\end{array}$ & $\begin{array}{c}17 \\
4.5 \\
28 \\
940 \\
51 \\
18 \\
\\
4.0 \\
7.9 \\
1.1 \\
3.5 \\
4.3 \\
0.57\end{array}$ & $\begin{array}{c}37 \\
6.4 \\
18 \\
1400 \\
86 \\
34 \\
\\
8.7 \\
6.6 \\
1.4 \\
4.0 \\
2.8 \\
0.47\end{array}$ & $\begin{array}{l}- \\
- \\
- \\
- \\
- \\
- \\
- \\
-\end{array}$ \\
\hline \multicolumn{6}{|c|}{ Anions on water wash by ion chromatography $( \pm 10 \%)$} \\
\hline $\begin{array}{l}\text { Inorganic } \\
\text { Bromide } \\
\text { Chloride } \\
\text { Chromate } \\
\text { Fluoride } \\
\text { Nitrate } \\
\text { Nitrite } \\
\text { Phosphate } \\
\text { Sulphate } \\
\text { Organic } \\
\text { Acetate } \\
\text { Citrate } \\
\text { Formate } \\
\text { Oxalate } \\
\text { Phthalate } \\
\text { Organic TICs } \\
\text { cis-Aconitate } \\
\text { trans-Aconitate } \\
\text { iso-Citrate } \\
\text { Malonate } \\
\text { Propionate }\end{array}$ & $\begin{array}{l}(\mathrm{mg} / \mathrm{Kg}) \\
(\mathrm{mg} / \mathrm{Kg}) \\
(\mathrm{mg} / \mathrm{Kg}) \\
(\mathrm{mg} / \mathrm{Kg}) \\
(\mathrm{mg} / \mathrm{Kg}) \\
(\mathrm{mg} / \mathrm{Kg}) \\
(\mathrm{mg} / \mathrm{Kg}) \\
(\mathrm{mg} / \mathrm{Kg}) \\
\\
(\mathrm{mg} / \mathrm{Kg}) \\
(\mathrm{mg} / \mathrm{Kg}) \\
(\mathrm{mg} / \mathrm{Kg}) \\
(\mathrm{mg} / \mathrm{Kg}) \\
(\mathrm{mg} / \mathrm{Kg}) \\
\\
(\mathrm{mg} / \mathrm{Kg}) \\
(\mathrm{mg} / \mathrm{Kg}) \\
(\mathrm{mg} / \mathrm{Kg}) \\
(\mathrm{mg} / \mathrm{Kg}) \\
(\mathrm{mg} / \mathrm{Kg}) \\
\end{array}$ & $\begin{array}{c}<5.2 \\
366 \\
- \\
233 \\
27.9 \\
576 \\
<21 \\
726\end{array}$ & $\begin{array}{l}<4.8 \\
536 \\
<10 \\
138 \\
<10 \\
1200 \\
<10 \\
1000 \\
\\
\\
<5 \\
<10 \\
<5 \\
<5 \\
<10 \\
\\
\\
\text { nd } \\
\text { nd } \\
\text { nd } \\
\text { nd } \\
\text { nd }\end{array}$ & $\begin{array}{l}<4.8 \\
494 \\
<10 \\
161 \\
<10 \\
944 \\
<10 \\
908 \\
\\
\\
<5 \\
<10 \\
<5 \\
23.9 \\
<10 \\
\\
\\
\text { nd } \\
\text { nd } \\
\text { nd } \\
\text { nd } \\
\text { nd } \\
\end{array}$ & $\begin{array}{l}- \\
- \\
- \\
- \\
- \\
-\end{array}$ \\
\hline \multicolumn{6}{|c|}{ Beta/gamma emitters $( \pm 10 \%)$} \\
\hline $\begin{array}{l}\text { Gross beta } \\
{ }^{14} \mathrm{C} \\
{ }^{60} \mathrm{Co} \\
{ }^{90} \mathrm{Sr}{ }^{80} \mathrm{Y} \\
{ }^{90} \mathrm{Tc} \\
{ }^{134} \mathrm{Cs} \\
{ }^{137} \mathrm{Cs} /{ }^{137} \mathrm{Ba} \\
{ }^{152} \mathrm{Eu} \\
{ }^{154} \mathrm{Eu} \\
{ }^{155} \mathrm{Eu} \\
{ }^{241} \mathrm{Pu}\end{array}$ & $\begin{array}{l}(\mathrm{Bq} / \mathrm{g}) \\
(\mathrm{Bq} / \mathrm{g}) \\
(\mathrm{Bq} / \mathrm{g}) \\
(\mathrm{Bq} / \mathrm{g}) \\
(\mathrm{Bq} / \mathrm{g}) \\
(\mathrm{Bq} / \mathrm{g}) \\
(\mathrm{Bq} / \mathrm{g}) \\
(\mathrm{Bq} / \mathrm{g}) \\
(\mathrm{Bq} / \mathrm{g}) \\
(\mathrm{Bq} / \mathrm{g}) \\
(\mathrm{Bq} / \mathrm{g})\end{array}$ & $\begin{array}{r}4.4 e+07 \\
- \\
7.7 e+04 \\
1.8 e+07 \\
4.7 e+01 \\
<5.1 e+02 \\
3.5 e+05 \\
7.3 e+04 \\
5.2 e+04 \\
<2.7 e+03 \\
5.0 e+04\end{array}$ & $\begin{array}{r}1.7 e+07 \\
- \\
2.3 e+04 \\
6.1 \mathrm{e}+06 \\
- \\
<1.1 \mathrm{e}+03 \\
2.1 \mathrm{e}+05 \\
1.9 \mathrm{e}+04 \\
1.5 \mathrm{e}+04 \\
<5.4 \mathrm{e}+03 \\
-\end{array}$ & $\begin{array}{r}3.5 \mathrm{e}+07 \\
- \\
3.2 \mathrm{e}+04 \\
1.2 \mathrm{e}+07 \\
- \\
<1.3 \mathrm{e}+03 \\
2.3 \mathrm{e}+05 \\
2.9 \mathrm{e}+04 \\
2.1 \mathrm{e}+04 \\
<7.1 \mathrm{e}+03 \\
-\end{array}$ & $\begin{array}{l}2.0 e+07 \\
1.7 e+01 \\
6.4 e+04 \\
1.2 e+07 \\
- \\
- \\
2.5 e+05 \\
3.8 e+04 \\
2.6 e+04 \\
3.8 e+03 \\
-\end{array}$ \\
\hline
\end{tabular}




\begin{tabular}{|c|c|c|c|c|c|}
\hline \multicolumn{2}{|l|}{$\begin{array}{l}\text { Characteristic } \\
\text { (Analysis) }\end{array}$} & $\begin{array}{c}\begin{array}{c}\text { T2-Center } \\
\text { sludge } \\
\text { (Winter 1996) }\end{array} \\
\end{array}$ & 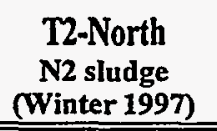 & $\begin{array}{c}\text { T2-South } \\
\text { S2 sludge } \\
\text { (Winter 1997) } \\
\end{array}$ & 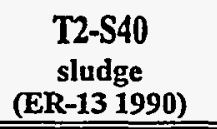 \\
\hline \multicolumn{6}{|c|}{ Alpha emitters $( \pm 10 \%)$} \\
\hline $\begin{array}{l}\text { Gross alpha } \\
{ }^{232} \mathrm{Th} \\
{ }^{233} \mathrm{U} \\
{ }^{234} \mathrm{U} \\
{ }^{235} \mathrm{U} \\
{ }^{238} \mathrm{U} \\
{ }^{237} \mathrm{~Np} \\
{ }^{241} \mathrm{Am} \\
{ }^{244} \mathrm{Cm} \\
{ }^{252} \mathrm{Cf} \\
{ }^{T o t a l} \mathrm{Pu} \text { alpha } \\
{ }^{238} \mathrm{Pu} \\
{ }^{242} \mathrm{Pu} /{ }^{240} \mathrm{Pu}\end{array}$ & $\begin{array}{l}(\mathrm{Bq} / \mathrm{g}) \\
(\mathrm{Bq} / \mathrm{g}) \\
(\mathrm{Bq} / \mathrm{g}) \\
(\mathrm{Bq} / \mathrm{g}) \\
(\mathrm{Bq} / \mathrm{g}) \\
(\mathrm{Bq} / \mathrm{g}) \\
(\mathrm{Bq} / \mathrm{g}) \\
(\mathrm{Bq} / \mathrm{g}) \\
(\mathrm{Bq} / \mathrm{g}) \\
(\mathrm{Bq} / \mathrm{g}) \\
(\mathrm{Bq} / \mathrm{g}) \\
(\mathrm{Bq} / \mathrm{g}) \\
(\mathrm{Bq} / \mathrm{g}) \\
(\mathrm{Bq} / \mathrm{g})\end{array}$ & $\begin{array}{r}5.3 \mathrm{e}+05 \\
3.8 \mathrm{e}+02 \\
7.8 \mathrm{e}+03 \\
9.5 \mathrm{e}+01 \\
0.5 \mathrm{e}+00 \\
2.6 \mathrm{e}+01 \\
1.2 \mathrm{e}+01 \\
2.6 \mathrm{e}+04 \\
4.6 \mathrm{e}+05 \\
<5.0 \mathrm{e}+02 \\
\\
3.4 \mathrm{e}+04 \\
2.3 \mathrm{e}+04 \\
1.0 \mathrm{e}+04 \\
-\end{array}$ & $\begin{array}{l}1.3 e+05 \\
9.8 \mathrm{e}+01 \\
2.8 \mathrm{e}+03 \\
3.0 \mathrm{e}+01 \\
0.2 \mathrm{e}+00 \\
1.0 \mathrm{e}+01 \\
- \\
5.0 \mathrm{e}+03 \\
1.1 \mathrm{e}+05 \\
- \\
1.2 \mathrm{e}+04 \\
7.9 \mathrm{e}+03 \\
3.8 \mathrm{e}+03 \\
-\end{array}$ & $\begin{array}{l}2.6 e+05 \\
2.2 e+02 \\
4.3 e+03 \\
4.9 e+01 \\
0.4 e+00 \\
1.7 e+01 \\
- \\
-8.9 e+03 \\
2.2 e+05 \\
- \\
2.2 e+04 \\
1.5 e+04 \\
7.2 e+03 \\
-\end{array}$ & $\begin{array}{l}2.5 e+05 \\
- \\
8.3 e+03 \\
- \\
- \\
- \\
- \\
<2.0 e+02^{1} \\
1.8 e+05^{1} \\
- \\
3.1 e+03 \\
5.1 e+03 \\
-\end{array}$ \\
\hline \multicolumn{6}{|c|}{ Uranium isotopics by TIMS $( \pm 0.5 \%)$} \\
\hline $\begin{array}{l}{ }^{233} \mathrm{U} \\
{ }^{234} \mathrm{U} \\
{ }^{235} \mathrm{U} \\
{ }^{236} \mathrm{U} \\
{ }^{238} \mathrm{U} /{ }^{235} \mathrm{U} \text { FEM } \\
{ }^{233} \mathrm{U} \\
{ }^{235} \mathrm{U}\end{array}$ & $\begin{array}{l}\text { (atom \%) } \\
\text { (atom \%) } \\
\text { (atom \%) } \\
\text { (atom \%) } \\
\text { (atom \%) } \\
\\
(\mathrm{mg} / \mathrm{Kg}) \\
(\mathrm{mg} / \mathrm{Kg})\end{array}$ & $\begin{array}{r}1.07 \\
0.02 \\
0.35 \\
0.01 \\
98.55 \\
56 \\
22 \\
7.2\end{array}$ & $\begin{array}{r}0.961 \\
0.016 \\
0.393 \\
0.007 \\
98.623 \\
59 \\
\\
7.8 \\
3.2\end{array}$ & $\begin{array}{c}0.912 \\
0.016 \\
0.402 \\
0.008 \\
98.662 \\
62 \\
\\
12 \\
5.4\end{array}$ & $\begin{array}{l}- \\
- \\
- \\
- \\
- \\
-\end{array}$ \\
\hline \multicolumn{6}{|c|}{ Uranium isotopics by ICP-MS ( $\pm 2 \%)$} \\
\hline $\begin{array}{l}{ }^{233} \mathrm{U} \\
{ }^{234} \mathrm{U} \\
{ }^{235} \mathrm{U} \\
{ }^{236} \mathrm{U} \\
{ }^{238} \mathrm{U} \\
{ }^{238} \mathrm{U} /{ }^{235} \mathrm{U} \text { FEM } \\
\\
{ }^{233} \mathrm{U} \\
{ }^{233} \mathrm{U}\end{array}$ & $\begin{array}{l}\text { (atom \%) } \\
\text { (atom \%) } \\
\text { (atom \%) } \\
\text { (atom \%) } \\
\text { (atom \%) }\end{array}$ & $\begin{array}{l}- \\
- \\
- \\
- \\
- \\
-\end{array}$ & $\begin{array}{r}0.950 \\
0.020 \\
0.399 \\
0.008 \\
98.623 \\
\\
60 \\
\\
7.1 \\
3.0\end{array}$ & $\begin{array}{r}0.915 \\
0.017 \\
0.396 \\
0.009 \\
98.664 \\
\\
62 \\
12 \\
5.2\end{array}$ & $\begin{array}{l}- \\
- \\
- \\
- \\
- \\
-\end{array}$ \\
\hline
\end{tabular}




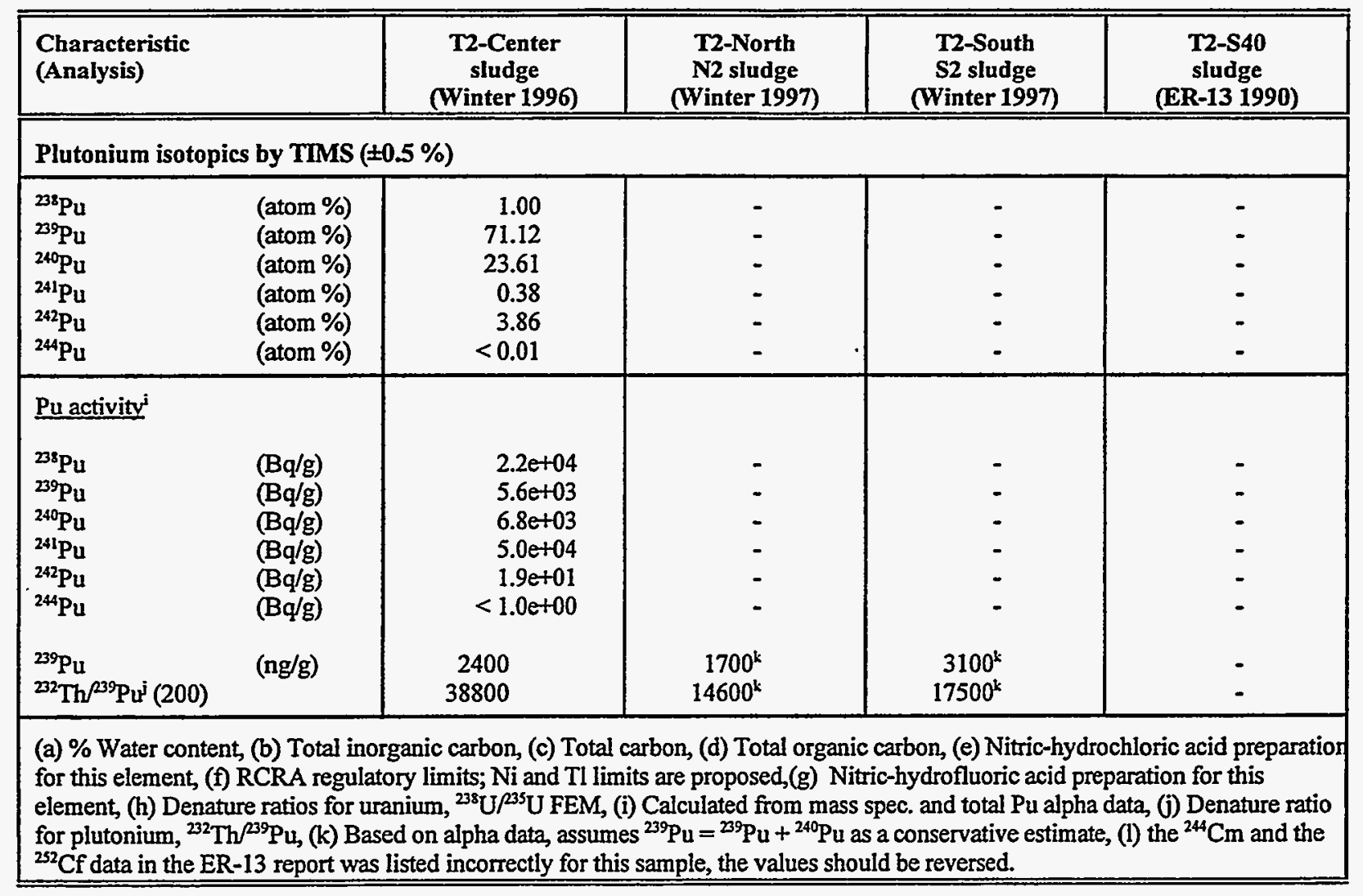


Table 10 Analytical data for OHF tank T3 sludge

\begin{tabular}{|c|c|c|c|c|c|c|}
\hline \multicolumn{3}{|c|}{$\begin{array}{l}\text { Characteristic } \\
\text { (Analysis) }\end{array}$} & $\begin{array}{c}\text { T3-Center } \\
\text { sludge } \\
\text { (Winter 1996) }\end{array}$ & $\begin{array}{c}\text { T3-North } \\
\text { N3 sludge } \\
\text { (Winter 1997) }\end{array}$ & $\begin{array}{c}\text { T3-South } \\
\text { S3 sludge } \\
\text { (Winter 1997) }\end{array}$ & $\begin{array}{c}\text { T3-S43 } \\
\text { sludge } \\
\text { (ER-13 1990) }\end{array}$ \\
\hline \multicolumn{7}{|c|}{ Physical properties and miscellaneous data } \\
\hline \multicolumn{3}{|c|}{$\begin{array}{l}\text { Request number } \\
\text { Sample number } \\
\text { pH (water wash) }\end{array}$} & $\begin{array}{r}7618 \mathrm{C} \\
960301-009 \\
11.5\end{array}$ & $\begin{array}{r}7973 A \\
970128-017 \\
11.4\end{array}$ & $\begin{array}{r}7973 \mathrm{~A} \\
970128-016 \\
11.0\end{array}$ & $:$ \\
\hline \multicolumn{2}{|c|}{$\begin{array}{l}\text { Water } \\
\text { Bulk density } \\
\text { TIC } \\
\text { TC }^{c} \\
\text { TOC }^{d}\end{array}$} & $\begin{array}{l}(\%) \\
(\mathrm{g} / \mathrm{mL}) \\
(\mathrm{mg} / \mathrm{Kg}) \\
(\mathrm{mg} / \mathrm{Kg}) \\
(\mathrm{mg} / \mathrm{Kg})\end{array}$ & $\begin{array}{c}60.4 \\
1.31 \\
12000 \\
16000 \\
4000 \\
\end{array}$ & $\begin{array}{c}71.0 \\
1.28 \\
5700 \\
12900 \\
7200 \\
\end{array}$ & $\begin{array}{c}71.2 \\
1.22 \\
4300 \\
12400 \\
8100 \\
\end{array}$ & $\begin{array}{c}63.6 \\
1.93 \\
- \\
- \\
9140 \\
\end{array}$ \\
\hline \multicolumn{7}{|c|}{ RCRA Metals ( $\pm 10 \%$ ) } \\
\hline & $\begin{array}{l}(100)^{f} \\
(100) \\
(2000) \\
(20) \\
(100) \\
(4) \\
(1000) \\
(100) \\
(20) \\
(18)\end{array}$ & $\begin{array}{l}(\mathrm{mg} / \mathrm{Kg}) \\
(\mathrm{mg} / \mathrm{Kg}) \\
(\mathrm{mg} / \mathrm{Kg}) \\
(\mathrm{mg} / \mathrm{Kg}) \\
(\mathrm{mg} / \mathrm{Kg}) \\
(\mathrm{mg} / \mathrm{Kg}) \\
(\mathrm{mg} / \mathrm{Kg}) \\
(\mathrm{mg} / \mathrm{Kg}) \\
(\mathrm{mg} / \mathrm{Kg}) \\
(\mathrm{mg} / \mathrm{Kg})\end{array}$ & $\begin{array}{c}<1.1 \\
<1.3 \\
69.6 \\
10.2 \\
51.8 \\
7.89 \\
50.0 \\
229 \\
<1.3 \\
<1.3\end{array}$ & $\begin{array}{c}<1.9 \\
- \\
74.9 \\
6.84 \\
60.5 \\
- \\
42.8 \\
- \\
- \\
-\end{array}$ & $\begin{array}{c}<1.7 \\
- \\
59.2 \\
8.09 \\
64.2 \\
- \\
79.4 \\
- \\
- \\
-\end{array}$ & $\begin{array}{c}0.15 \\
<3 \\
76 \\
8.5 \\
69 \\
40 \\
57 \\
300 \\
0.74 \\
<0.6\end{array}$ \\
\hline \multicolumn{7}{|c|}{ Process metals ( $\pm 10 \%)$} \\
\hline $\begin{array}{l}\mathrm{Al} \\
\mathrm{B} \\
\mathrm{Be} \\
\mathrm{Ca} \\
\mathrm{Co} \\
\mathrm{Cu} \\
\mathrm{Cs} \\
\mathrm{Fe} \\
\mathrm{K} \\
\mathrm{Mg} \\
\mathrm{Mn} \\
\mathrm{Na} \\
\mathrm{P} \\
\mathrm{Sb}^{\mathrm{e}} \\
\mathrm{Si}^{\mathrm{g}} \\
\mathrm{Sr} \\
\mathrm{Th} \\
\mathrm{U} \\
\mathrm{V} \\
\mathrm{Zn}\end{array}$ & & $\begin{array}{l}(\mathrm{mg} / \mathrm{Kg}) \\
(\mathrm{mg} / \mathrm{Kg}) \\
(\mathrm{mg} / \mathrm{Kg}) \\
(\mathrm{mg} / \mathrm{Kg}) \\
(\mathrm{mg} / \mathrm{Kg}) \\
(\mathrm{mg} / \mathrm{Kg}) \\
(\mathrm{mg} / \mathrm{Kg}) \\
(\mathrm{mg} / \mathrm{Kg}) \\
(\mathrm{mg} / \mathrm{Kg}) \\
(\mathrm{mg} / \mathrm{Kg}) \\
(\mathrm{mg} / \mathrm{Kg}) \\
(\mathrm{mg} / \mathrm{Kg}) \\
(\mathrm{mg} / \mathrm{Kg}) \\
(\mathrm{mg} / \mathrm{Kg}) \\
(\mathrm{mg} / \mathrm{Kg}) \\
(\mathrm{mg} / \mathrm{Kg}) \\
(\mathrm{mg} / \mathrm{Kg}) \\
(\mathrm{mg} / \mathrm{Kg}) \\
(\mathrm{mg} / \mathrm{Kg}) \\
(\mathrm{mg} / \mathrm{Kg})\end{array}$ & $\begin{array}{c}15600 \\
31.8 \\
<2.9 \\
37900 \\
5.53 \\
64.3 \\
5.66 \\
7790 \\
6140 \\
3570 \\
199 \\
18800 \\
4870 \\
<19 \\
32500 \\
282 \\
77500 \\
5920 \\
<7.3 \\
151 \\
\end{array}$ & $\begin{array}{c}8500 \\
12.7 \\
12.5 \\
27000 \\
8.87 \\
41.8 \\
- \\
4890 \\
5590 \\
2880 \\
137 \\
15600 \\
1220 \\
<19 \\
- \\
182 \\
36100 \\
3170 \\
8.29 \\
85.0 \\
\end{array}$ & $\begin{array}{c}11100 \\
17.4 \\
23.4 \\
24300 \\
11.2 \\
50.3 \\
- \\
4420 \\
5200 \\
2780 \\
164 \\
15600 \\
1550 \\
<17 \\
- \\
204 \\
44600 \\
3910 \\
10.2 \\
105 \\
\end{array}$ & $\begin{array}{r}- \\
- \\
- \\
- \\
- \\
- \\
- \\
- \\
- \\
- \\
- \\
- \\
- \\
-\end{array}$ \\
\hline
\end{tabular}




\begin{tabular}{|c|c|c|c|c|c|}
\hline $\begin{array}{l}\text { Characteristic } \\
\text { (Analysis) }\end{array}$ & & $\begin{array}{c}\begin{array}{c}\text { T3-Center } \\
\text { sludge } \\
\text { (Winter 1996) }\end{array} \\
\end{array}$ & $\begin{array}{c}\text { T3-North } \\
\text { N3 sludge } \\
\text { (Winter 1997) } \\
\end{array}$ & $\begin{array}{c}\text { T3-South } \\
\text { S3 sludge } \\
\text { (Winter 1997) } \\
\end{array}$ & $\begin{array}{c}\text { T3-S43 } \\
\text { sludge } \\
\text { (ER-13 1990) } \\
\end{array}$ \\
\hline \multicolumn{6}{|c|}{ Semi-quantitative elements by ICP-MS ( $\pm 30-50 \%$ ) } \\
\hline $\begin{array}{l}\mathrm{Bi} \\
\mathrm{Ga} \\
\mathrm{I} \\
\mathrm{Li} \\
\mathrm{Te} \\
\mathrm{Zr} \\
\text { Lanthanides } \\
(57) \mathrm{La} \\
(58) \mathrm{Ce} \\
(59) \mathrm{Pr} \\
(60) \mathrm{Nd} \\
(62) \mathrm{Sm} \\
(63) \mathrm{Eu}\end{array}$ & $\begin{array}{l}\text { (mg/Kg) } \\
(\mathrm{mg} / \mathrm{Kg}) \\
(\mathrm{mg} / \mathrm{Kg}) \\
(\mathrm{mg} / \mathrm{Kg}) \\
(\mathrm{mg} / \mathrm{Kg}) \\
(\mathrm{mg} / \mathrm{Kg}) \\
\\
(\mathrm{mg} / \mathrm{Kg}) \\
(\mathrm{mg} / \mathrm{Kg}) \\
(\mathrm{mg} / \mathrm{Kg}) \\
(\mathrm{mg} / \mathrm{Kg}) \\
(\mathrm{mg} / \mathrm{Kg}) \\
(\mathrm{mg} / \mathrm{Kg})\end{array}$ & $\begin{array}{l}- \\
- \\
- \\
- \\
- \\
- \\
- \\
- \\
- \\
-\end{array}$ & $\begin{array}{c}5.2 \\
6.9 \\
31 \\
260 \\
64 \\
22 \\
\\
9.4 \\
17 \\
1.8 \\
10 \\
2.0 \\
0.63\end{array}$ & $\begin{array}{c}9.4 \\
6.7 \\
25 \\
510 \\
66 \\
31 \\
\\
8.9 \\
14 \\
2.1 \\
8.6 \\
1.8 \\
0.61\end{array}$ & $\begin{array}{l}- \\
- \\
- \\
- \\
- \\
- \\
- \\
- \\
- \\
-\end{array}$ \\
\hline \multicolumn{6}{|c|}{ Anions on water wash by ion chromatography $( \pm 10 \%)$} \\
\hline $\begin{array}{l}\text { Inorganic } \\
\text { Bromide } \\
\text { Chloride } \\
\text { Chromate } \\
\text { Fluoride } \\
\text { Nitrate } \\
\text { Nitrite } \\
\text { Phosphate } \\
\text { Sulphate } \\
\text { Organic } \\
\text { Acetate } \\
\text { Citrate } \\
\text { Formate } \\
\text { Oxalate } \\
\text { Phthalate } \\
\text { Organic TICs } \\
\text { cis-Aconitate } \\
\text { trans-Aconitate } \\
\text { iso-Citrate } \\
\text { Malonate } \\
\text { Propionate } \\
\text { Unknown }\end{array}$ & $\begin{array}{l}\text { (mg/Kg) } \\
\text { (mg/Kg) } \\
(\mathrm{mg} / \mathrm{Kg}) \\
(\mathrm{mg} / \mathrm{Kg}) \\
(\mathrm{mg} / \mathrm{Kg}) \\
(\mathrm{mg} / \mathrm{Kg}) \\
(\mathrm{mg} / \mathrm{Kg}) \\
(\mathrm{mg} / \mathrm{Kg}) \\
\\
(\mathrm{mg} / \mathrm{Kg}) \\
(\mathrm{mg} / \mathrm{Kg}) \\
(\mathrm{mg} / \mathrm{Kg}) \\
(\mathrm{mg} / \mathrm{Kg}) \\
(\mathrm{mg} / \mathrm{Kg}) \\
\\
(\mathrm{mg} / \mathrm{Kg}) \\
(\mathrm{mg} / \mathrm{Kg}) \\
(\mathrm{mg} / \mathrm{Kg}) \\
(\mathrm{mg} / \mathrm{Kg}) \\
(\mathrm{mg} / \mathrm{Kg}) \\
(\mathrm{mg} / \mathrm{Kg})\end{array}$ & $\begin{array}{r}<43 \\
947 \\
- \\
257 \\
4250 \\
4670 \\
<174 \\
2960 \\
\\
. \\
- \\
- \\
- \\
- \\
- \\
- \\
- \\
- \\
- \\
-\end{array}$ & $\begin{array}{c}40.1 \\
1160 \\
32.8 \\
198 \\
5000 \\
8240 \\
<10 \\
3280 \\
\\
524 \\
<10 \\
460 \\
1160 \\
<10 \\
\\
\text { nd } \\
\text { nd } \\
\text { nd } \\
\text { nd } \\
50 \\
110 .(5)\end{array}$ & $\begin{array}{c}19.7 \\
1130 \\
37.2 \\
207 \\
4320 \\
8530 \\
<10 \\
3430 \\
\\
533 \\
<10 \\
450 \\
1450 \\
<10 \\
\\
\text { nd } \\
\text { nd } \\
\text { nd } \\
\text { nd } \\
50 \\
130 .(6)\end{array}$ & $\begin{array}{l}- \\
- \\
- \\
- \\
- \\
- \\
- \\
-\end{array}$ \\
\hline \multicolumn{6}{|c|}{ Beta/gamma emitters $( \pm 10 \%)$} \\
\hline $\begin{array}{l}\text { Gross beta } \\
{ }^{14} \mathrm{C} \\
{ }^{60} \mathrm{Co} \\
{ }^{90} \mathrm{Sr} /{ }^{\circ 0} \mathrm{Y} \\
{ }^{99} \mathrm{Tc} \\
{ }^{134} \mathrm{Cs} \\
{ }^{137} \mathrm{Cs} /{ }^{137} \mathrm{~m} \mathrm{Ba} \\
{ }^{152} \mathrm{Eu} \\
{ }^{154} \mathrm{Eu} \\
{ }^{155} \mathrm{Eu} \\
{ }^{241} \mathrm{Pu}\end{array}$ & $\begin{array}{l}\text { (Bq/g) } \\
(\mathrm{Bq} / \mathrm{g}) \\
(\mathrm{Bq} / \mathrm{g}) \\
(\mathrm{Bq} / \mathrm{g}) \\
(\mathrm{Bq} / \mathrm{g}) \\
(\mathrm{Bq} / \mathrm{g}) \\
(\mathrm{Bq} / \mathrm{g}) \\
(\mathrm{Bq} / \mathrm{g}) \\
(\mathrm{Bq} / \mathrm{g}) \\
(\mathrm{Bq} / \mathrm{g}) \\
(\mathrm{Bq} / \mathrm{g})\end{array}$ & $\begin{array}{r}2.3 e+07 \\
- \\
1.0 e+05 \\
8.5 e+06 \\
3.3 e+01 \\
<7.1 e+02 \\
1.6 e+06 \\
5.6 e+04 \\
3.4 e+04 \\
5.9 e+03 \\
1.1 e+04\end{array}$ & $\begin{array}{l}1.3 e+07 \\
- \\
4.5 e+04 \\
4.7 e+06 \\
- \\
<1.8 e+03 \\
1.1 e+06 \\
3.0 e+04 \\
2.0 e+04 \\
<6.2 e+03 \\
-\end{array}$ & $\begin{array}{r}2.2 e+07 \\
- \\
5.5 e+04 \\
7.5 e+06 \\
- \\
<1.7 e+03 \\
9.9 e+05 \\
4.0 e+04 \\
2.2 e+04 \\
<6.7 e+03 \\
-\end{array}$ & $\begin{array}{l}2.5 e+07 \\
7.6 e+02 \\
1.6 e+05 \\
8.1 e+06 \\
- \\
- \\
1.3 e+06 \\
5.1 e+04 \\
5.3 e+04 \\
- \\
-\end{array}$ \\
\hline
\end{tabular}




\begin{tabular}{|c|c|c|c|c|c|}
\hline $\begin{array}{l}\text { Characteristic } \\
\text { (Analysis) }\end{array}$ & & $\begin{array}{c}\begin{array}{c}\text { T3-Center } \\
\text { sludge } \\
\text { (Winter 1996) }\end{array} \\
\end{array}$ & 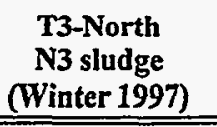 & 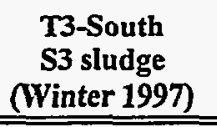 & 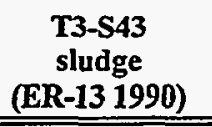 \\
\hline \multicolumn{6}{|c|}{ Alpha emitters $( \pm 10 \%)$} \\
\hline $\begin{array}{l}\text { Gross alpha } \\
{ }^{232} \mathrm{Th} \\
{ }^{233} \mathrm{U} \\
{ }^{234} \mathrm{U} \\
{ }^{235} \mathrm{U} \\
{ }^{238} \mathrm{U} \\
{ }^{237} \mathrm{~Np} \\
{ }^{241} \mathrm{Am} \\
{ }^{244} \mathrm{Cm} \\
{ }^{252} \mathrm{Cf} \\
{ }^{\text {Total }} \mathrm{Pu} \text { alpha } \\
{ }^{238} \mathrm{Pu} \\
{ }^{242} \mathrm{Pu} /{ }^{240} \mathrm{Pu}\end{array}$ & $\begin{array}{l}(\mathrm{Bq} / \mathrm{g}) \\
(\mathrm{Bq} / \mathrm{g}) \\
(\mathrm{Bq} / \mathrm{g}) \\
(\mathrm{Bq} / \mathrm{g}) \\
(\mathrm{Bq} / \mathrm{g}) \\
(\mathrm{Bq} / \mathrm{g}) \\
(\mathrm{Bq} / \mathrm{g}) \\
(\mathrm{Bq} / \mathrm{g}) \\
(\mathrm{Bq} / \mathrm{g}) \\
(\mathrm{Bq} / \mathrm{g}) \\
: \\
(\mathrm{Bq} / \mathrm{g}) \\
(\mathrm{Bq} / \mathrm{g}) \\
(\mathrm{Bq} / \mathrm{g}) \\
(\mathrm{Bq} / \mathrm{g})\end{array}$ & $\begin{array}{r}3.0 e+05 \\
3.2 e+02 \\
1.5 e+04 \\
1.3 e+02 \\
1.7 e+00 \\
7.3 e+01 \\
8.9 e+00 \\
1.5 e+04 \\
2.5 e+05 \\
<3.0 e+02 \\
1.7 e+04 \\
1.1 e+04 \\
6.5 e+03 \\
-\end{array}$ & $\begin{array}{l}1.6 e+05 \\
1.5 e+02 \\
7.7 e+03 \\
9.3 e+01 \\
1.0 e+00 \\
3.9 e+01 \\
- \\
2.0 e+03 \\
1.4 e+05 \\
- \\
9.8 e+03 \\
6.2 e+03 \\
3.6 e+03 \\
-\end{array}$ & $\begin{array}{l}2.4 e+05 \\
1.8 e+02 \\
9.9 e+03 \\
1.2 e+02 \\
1.2 e+00 \\
4.8 e+01 \\
- \\
1.4 e+04 \\
1.9 e+05 \\
- \\
1.5 e+04 \\
1.1 e+04 \\
4.2 e+03 \\
-\end{array}$ & $\begin{array}{l}2.0 e+05 \\
- \\
8.3 e+03 \\
- \\
- \\
- \\
- \\
- \\
1.8 e+05 \\
<2.0 e+02 \\
- \\
1.4 e+04 \\
5.3 e+03 \\
-\end{array}$ \\
\hline \multicolumn{6}{|c|}{ Uranium isotopics by TIMS ( $\pm 0.5 \%)$} \\
\hline $\begin{array}{l}{ }^{233} U \\
{ }^{234} U \\
{ }^{235} U \\
{ }^{236} U \\
{ }^{238} U /{ }^{235} U \text { FEM } \\
{ }^{233} U \\
{ }^{235} U\end{array}$ & $\begin{array}{l}\text { (atom \%) } \\
\text { (atom \%) } \\
\text { (atom \%) } \\
\text { (atom \%) } \\
\text { (atom \%) } \\
\\
(\mathrm{mg} / \mathrm{Kg}) \\
(\mathrm{mg} / \mathrm{Kg})\end{array}$ & $\begin{array}{r}0.74 \\
0.01 \\
0.40 \\
0.01 \\
98.84 \\
\\
72 \\
\\
43 \\
23\end{array}$ & $\begin{array}{r}0.693 \\
0.013 \\
0.430 \\
0.007 \\
98.857 \\
74 \\
\\
22 \\
14\end{array}$ & $\begin{array}{r}0.723 \\
0.013 \\
0.440 \\
0.007 \\
98.817 \\
71 \\
\\
28 \\
17\end{array}$ & $\begin{array}{ll}- & \cdot \\
- \\
- \\
- \\
- \\
- \\
- \\
-\end{array}$ \\
\hline \multicolumn{6}{|c|}{ Uranium isotopics by ICP-MS ( $\pm 2 \%$ ) } \\
\hline $\begin{array}{l}{ }^{233} U \\
{ }^{234} U \\
{ }^{235} U \\
{ }^{236} U \\
{ }^{238} U \\
{ }^{238} U F^{235} M^{\mathrm{h}} \\
{ }^{233} U \\
{ }^{235} U\end{array}$ & $\begin{array}{l}\text { (atom \%) } \\
\text { (atom \%) } \\
\text { (atom \%) } \\
\text { (atom \%) } \\
\text { (atom \%) } \\
\\
(\mathrm{mg} / \mathrm{Kg}) \\
(\mathrm{mg} / \mathrm{Kg})\end{array}$ & $\begin{array}{l}- \\
- \\
- \\
- \\
- \\
- \\
-\end{array}$ & $\begin{array}{r}0.688 \\
0.013 \\
0.433 \\
0.008 \\
98.859 \\
74 \\
\\
22 \\
14\end{array}$ & $\begin{array}{l}0.738 \\
0.012 \\
0.442 \\
0.007 \\
98.800 \\
70 \\
\\
28 \\
17\end{array}$ & $\begin{array}{l}- \\
- \\
- \\
- \\
- \\
-\end{array}$ \\
\hline
\end{tabular}




\begin{tabular}{|c|c|c|c|c|c|}
\hline $\begin{array}{l}\text { Characteristic } \\
\text { (Analysis) }\end{array}$ & & $\begin{array}{c}\text { T3-Center } \\
\text { sludge } \\
\text { (Winter 1996) } \\
\end{array}$ & $\begin{array}{c}\text { T3-North } \\
\text { N3 sludge } \\
\text { (Winter 1997) } \\
\end{array}$ & $\begin{array}{c}\text { T3-South } \\
\text { S3 sludge } \\
\text { (Winter 1997) } \\
\end{array}$ & 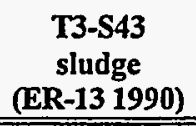 \\
\hline \multicolumn{6}{|c|}{ Plutonium isotopics by TIMS $( \pm 0.5 \%)$} \\
\hline $\begin{array}{l}{ }^{238} \mathrm{Pu} \\
{ }^{239} \mathrm{Pu} \\
{ }^{240} \mathrm{Pu} \\
{ }^{241} \mathrm{Pu} \\
{ }^{244} \mathrm{Pu} \\
{ }^{44} \mathrm{Pu}\end{array}$ & $\begin{array}{l}\text { (atom \%) } \\
\text { (atom \%) } \\
\text { (atom \%) } \\
\text { (atom \%) } \\
\text { (atom \%) } \\
\text { (atom \%) }\end{array}$ & $\begin{array}{r}0.68 \\
69.02 \\
26.86 \\
0.14 \\
3.30 \\
<0.01\end{array}$ & $\begin{array}{l}- \\
- \\
- \\
-\end{array}$ & $\begin{array}{l}- \\
- \\
- \\
-\end{array}$ & $\begin{array}{l}- \\
- \\
- \\
-\end{array}$ \\
\hline \multicolumn{6}{|l|}{ Pu activity } \\
\hline $\begin{array}{l}{ }^{238} \mathrm{Pu} \\
{ }^{239} \mathrm{Pu} \\
{ }^{240} \mathrm{Pu} \\
{ }^{241} \mathrm{Pu} \\
{ }^{244} \mathrm{Pu}\end{array}$ & $\begin{array}{l}(\mathrm{Bq} / \mathrm{g}) \\
(\mathrm{Bq} / \mathrm{g}) \\
(\mathrm{Bq} / \mathrm{g}) \\
(\mathrm{Bq} / \mathrm{g}) \\
(\mathrm{Bq} / \mathrm{g}) \\
(\mathrm{Bq} / \mathrm{g})\end{array}$ & $\begin{array}{r}8.9 \mathrm{e}+03 \\
3.3 \mathrm{e}+03 \\
4.7 \mathrm{e}+03 \\
1.1 \mathrm{e}+04 \\
1.0 \mathrm{e}+01 \\
<1.0 \mathrm{e}+00\end{array}$ & $\begin{array}{l}- \\
- \\
-\end{array}$ & $\begin{array}{l}- \\
- \\
-\end{array}$ & $\begin{array}{l}- \\
- \\
- \\
-\end{array}$ \\
\hline${ }^{{ }^{239} \mathrm{Pu}} \mathrm{Th} /{ }^{399} \mathrm{Puj}(200)$ & (ng/g) & $\begin{array}{r}1440 \\
53800\end{array}$ & $\begin{array}{r}1600^{k} \\
23000^{k}\end{array}$ & $\begin{array}{r}1800^{k} \\
24400^{k}\end{array}$ & - \\
\hline
\end{tabular}


Table 11 Analytical data for OHF tank T4 sludge

\begin{tabular}{|c|c|c|c|c|c|}
\hline \multicolumn{2}{|l|}{$\begin{array}{l}\text { Characteristic } \\
\text { (Analysis) }\end{array}$} & $\begin{array}{c}\begin{array}{c}\text { T4-Center } \\
\text { sludge } \\
\text { (Winter 1996) }\end{array} \\
\end{array}$ & $\begin{array}{c}\text { T4-North } \\
\text { N4 sludge } \\
\text { (Winter 1997) } \\
\end{array}$ & $\begin{array}{c}\text { T4-South } \\
\text { S4 sludge } \\
\text { (Winter 1997) }\end{array}$ & $\begin{array}{c}\begin{array}{c}\text { T4-S46 } \\
\text { sludge } \\
\text { (ER-13 1990) }\end{array} \\
\end{array}$ \\
\hline \multicolumn{6}{|c|}{ Physical properties and miscellaneous data } \\
\hline \multicolumn{2}{|l|}{$\begin{array}{l}\text { Request number } \\
\text { Sample number } \\
\text { pH (water wash) }\end{array}$} & $\begin{array}{r}7618 \mathrm{~B} \\
960227-093 \\
10.4\end{array}$ & $\begin{array}{r}7973 \mathrm{C} \\
970128-111 \\
9.8\end{array}$ & $\begin{array}{r}7973 \mathrm{C} \\
970128-110 \\
9.9\end{array}$ & $\begin{array}{l}- \\
-\end{array}$ \\
\hline $\begin{array}{l}\text { Water } \\
\text { Bulk density } \\
\text { TIC } \\
\text { TC } \\
\text { TOC }^{\mathrm{c}}\end{array}$ & $\begin{array}{l}(\%) \\
(\mathrm{g} / \mathrm{mL}) \\
(\mathrm{mg} / \mathrm{Kg}) \\
(\mathrm{mg} / \mathrm{Kg}) \\
(\mathrm{mg} / \mathrm{Kg})\end{array}$ & $\begin{array}{c}72.2 \\
1.21 \\
5200 \\
15000 \\
9800\end{array}$ & $\begin{array}{c}80.0 \\
1.13 \\
\cdot 2400 \\
7100 \\
4700\end{array}$ & $\begin{array}{c}79.8 \\
1.25 \\
2700 \\
7200 \\
4500 \\
\end{array}$ & $\begin{array}{c}74.7 \\
1.24 \\
- \\
- \\
4620\end{array}$ \\
\hline \multicolumn{6}{|c|}{ RCRA Metals ( $\pm 10 \%)$} \\
\hline $\begin{array}{ll}\mathrm{Ag}^{\circ} & (100)^{\mathrm{r}} \\
\mathrm{As} & (100) \\
\mathrm{Ba} & (2000) \\
\mathrm{Cd} & (20) \\
\mathrm{Cr} & (100) \\
\mathrm{Hg} & (4) \\
\mathrm{Ni} & (1000) \\
\mathrm{Pb} & (100) \\
\mathrm{Se} & (20) \\
\mathrm{Tl} & (18)\end{array}$ & $\begin{array}{l}(\mathrm{mg} / \mathrm{Kg}) \\
(\mathrm{mg} / \mathrm{Kg}) \\
(\mathrm{mg} / \mathrm{Kg}) \\
(\mathrm{mg} / \mathrm{Kg}) \\
(\mathrm{mg} / \mathrm{Kg}) \\
(\mathrm{mg} / \mathrm{Kg}) \\
(\mathrm{mg} / \mathrm{Kg}) \\
(\mathrm{mg} / \mathrm{Kg}) \\
(\mathrm{mg} / \mathrm{Kg}) \\
(\mathrm{mg} / \mathrm{Kg})\end{array}$ & $\begin{array}{c}<1.0 \\
<1.2 \\
26.5 \\
16.4 \\
118 \\
15.1 \\
134 \\
598 \\
<1.2 \\
<1.2\end{array}$ & $\begin{array}{c}<1.9 \\
- \\
27.0 \\
8.63 \\
71.4 \\
- \\
139 \\
- \\
- \\
-\end{array}$ & $\begin{array}{c}<2.0 \\
- \\
25.1 \\
9.52 \\
79.8 \\
- \\
93.9 \\
- \\
- \\
-\end{array}$ & $\begin{array}{c}1.7 \\
<4 \\
<50 \\
10 \\
102 \\
585 \\
160 \\
510 \\
1.5 \\
0.73 \\
\end{array}$ \\
\hline \multicolumn{6}{|c|}{ Process metals ( $\pm 10 \%$ ) } \\
\hline $\begin{array}{l}\mathrm{Al} \\
\mathrm{B} \\
\mathrm{Be} \\
\mathrm{Ca} \\
\mathrm{Co} \\
\mathrm{Cu} \\
\mathrm{Cs} \\
\mathrm{Fe} \\
\mathrm{K} \\
\mathrm{Mg} \\
\mathrm{Mn} \\
\mathrm{Na} \\
\mathrm{P} \\
\mathrm{Sb}^{\mathrm{e}} \\
\mathrm{Si}^{\mathbf{2}} \\
\mathrm{Sr} \\
\mathrm{Th} \\
\mathrm{U} \\
\mathrm{V} \\
\mathrm{Zn}\end{array}$ & $\begin{array}{l}(\mathrm{mg} / \mathrm{Kg}) \\
(\mathrm{mg} / \mathrm{Kg}) \\
(\mathrm{mg} / \mathrm{Kg}) \\
(\mathrm{mg} / \mathrm{Kg}) \\
(\mathrm{mg} / \mathrm{Kg}) \\
(\mathrm{mg} / \mathrm{Kg}) \\
(\mathrm{mg} / \mathrm{Kg}) \\
(\mathrm{mg} / \mathrm{Kg}) \\
(\mathrm{mg} / \mathrm{Kg}) \\
(\mathrm{mg} / \mathrm{Kg}) \\
(\mathrm{mg} / \mathrm{Kg}) \\
(\mathrm{mg} / \mathrm{Kg}) \\
(\mathrm{mg} / \mathrm{Kg}) \\
(\mathrm{mg} / \mathrm{Kg}) \\
(\mathrm{mg} / \mathrm{Kg}) \\
(\mathrm{mg} / \mathrm{Kg}) \\
(\mathrm{mg} / \mathrm{Kg}) \\
(\mathrm{mg} / \mathrm{Kg}) \\
(\mathrm{mg} / \mathrm{Kg}) \\
(\mathrm{mg} / \mathrm{Kg})\end{array}$ & $\begin{array}{c}9320 \\
49.7 \\
<2.7 \\
20600 \\
11.0 \\
293 \\
5.53 \\
3150 \\
2080 \\
1730 \\
472 \\
7400 \\
6880 \\
<17 \\
4570 \\
334 \\
124000 \\
7870 \\
<6.8 \\
183 \\
\end{array}$ & $\begin{array}{c}17900 \\
15.5 \\
53.7 \\
9950 \\
14.1 \\
72.1 \\
- \\
3540 \\
2240 \\
1530 \\
360 \\
5330 \\
2320 \\
51.7 \\
- \\
175 \\
39100 \\
3130 \\
4.31 \\
116\end{array}$ & $\begin{array}{c}17700 \\
17.0 \\
64.1 \\
10400 \\
22.5 \\
70.6 \\
- \\
2870 \\
2350 \\
1230 \\
277 \\
5870 \\
2290 \\
45.9 \\
- \\
204 \\
45200 \\
3270 \\
4.61 \\
122 \\
\end{array}$ & $\begin{array}{r}- \\
- \\
- \\
- \\
- \\
- \\
- \\
- \\
- \\
- \\
- \\
- \\
- \\
- \\
- \\
\end{array}$ \\
\hline
\end{tabular}




\begin{tabular}{|c|c|c|c|c|c|}
\hline $\begin{array}{l}\text { Characteristic } \\
\text { (Analysis) }\end{array}$ & & $\begin{array}{c}\begin{array}{c}\text { T4-Center } \\
\text { sludge } \\
\text { (Winter 1996) }\end{array} \\
\end{array}$ & $\begin{array}{c}\text { T4-North } \\
\text { N4 sludge } \\
\text { (Winter 1997) } \\
\end{array}$ & $\begin{array}{c}\text { T4-South } \\
\text { S4 sludge } \\
\text { (Winter 1997) } \\
\end{array}$ & 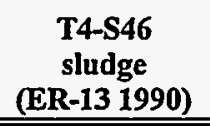 \\
\hline \multicolumn{6}{|c|}{ Semi-quantitative elements by ICP-MS ( $\pm 30-50 \%$ ) } \\
\hline $\begin{array}{l}\mathrm{Bi} \\
\mathrm{Ga} \\
\mathrm{I} \\
\mathrm{Li} \\
\mathrm{Te} \\
\mathrm{Zr} \\
\mathrm{Lanthanides} \\
\text { (57) } \mathrm{La} \\
\text { (58) } \mathrm{Ce} \\
\text { (59) } \mathrm{Pr} \\
\text { (60) } \mathrm{Nd} \\
\text { (62) } \mathrm{Sm} \\
\text { (63) } \mathrm{Eu}\end{array}$ & $\begin{array}{l}(\mathrm{mg} / \mathrm{Kg}) \\
(\mathrm{mg} / \mathrm{Kg}) \\
(\mathrm{mg} / \mathrm{Kg}) \\
(\mathrm{mg} / \mathrm{Kg}) \\
(\mathrm{mg} / \mathrm{Kg}) \\
(\mathrm{mg} / \mathrm{Kg}) \\
(\mathrm{mg} / \mathrm{Kg}) \\
(\mathrm{mg} / \mathrm{Kg}) \\
(\mathrm{mg} / \mathrm{Kg}) \\
(\mathrm{mg} / \mathrm{Kg}) \\
(\mathrm{mg} / \mathrm{Kg}) \\
(\mathrm{mg} / \mathrm{Kg})\end{array}$ & $\begin{array}{l}- \\
- \\
- \\
- \\
- \\
- \\
- \\
- \\
-\end{array}$ & $\begin{array}{c}33 \\
5.1 \\
51 \\
1500 \\
71 \\
32 \\
8.0 \\
10 \\
1.3 \\
6.0 \\
1.7 \\
0.70\end{array}$ & $\begin{array}{c}32 \\
5.3 \\
21 \\
1800 \\
79 \\
37 \\
\\
9.3 \\
6.3 \\
1.5 \\
4.4 \\
2.0 \\
0.56\end{array}$ & $\begin{array}{l}- \\
- \\
- \\
- \\
- \\
- \\
-\end{array}$ \\
\hline \multicolumn{6}{|c|}{ Anions on water wash by ion chromatography $( \pm 10 \%)$} \\
\hline $\begin{array}{l}\text { Inorganic } \\
\text { Bromide } \\
\text { Chloride } \\
\text { Chromate } \\
\text { Fluoride } \\
\text { Nitrate } \\
\text { Nitrite } \\
\text { Phosphate } \\
\text { Sulphate } \\
\text { Organic } \\
\text { Acetate } \\
\text { Citrate } \\
\text { Formate } \\
\text { Oxalate } \\
\text { Phthalate } \\
\\
\text { Organic TICs } \\
\text { cis-Aconitate } \\
\text { trans-Aconitate } \\
\text { iso-Citrate } \\
\text { Malonate } \\
\text { Propionate }\end{array}$ & $\begin{array}{l}\text { (mg/Kg) } \\
(\mathrm{mg} / \mathrm{Kg}) \\
(\mathrm{mg} / \mathrm{Kg}) \\
(\mathrm{mg} / \mathrm{Kg}) \\
(\mathrm{mg} / \mathrm{Kg}) \\
(\mathrm{mg} / \mathrm{Kg}) \\
(\mathrm{mg} / \mathrm{Kg}) \\
(\mathrm{mg} / \mathrm{Kg}) \\
\\
(\mathrm{mg} / \mathrm{Kg}) \\
(\mathrm{mg} / \mathrm{Kg}) \\
(\mathrm{mg} / \mathrm{Kg}) \\
(\mathrm{mg} / \mathrm{Kg}) \\
(\mathrm{mg} / \mathrm{Kg}) \\
\\
(\mathrm{mg} / \mathrm{Kg}) \\
(\mathrm{mg} / \mathrm{Kg}) \\
(\mathrm{mg} / \mathrm{Kg}) \\
(\mathrm{mg} / \mathrm{Kg}) \\
(\mathrm{mg} / \mathrm{Kg})\end{array}$ & $\begin{array}{r}<41 \\
401 \\
- \\
272 \\
1470 \\
1460 \\
<165 \\
1210\end{array}$ & $\begin{array}{c}<4.8 \\
461 \\
17.9 \\
112 \\
2020 \\
2610 \\
<10 \\
1190 \\
\\
\\
197 \\
<10 \\
135 \\
913 \\
<10 \\
\\
\\
\text { nd } \\
\text { nd } \\
\text { nd } \\
\text { nd } \\
10 \\
\end{array}$ & $\begin{array}{c}<4.6 \\
462 \\
19.8 \\
133 \\
1660 \\
2920 \\
<10 \\
1360 \\
\\
\\
244 \\
<10 \\
148 \\
649 \\
<10 \\
\\
\\
\text { nd } \\
\text { nd } \\
\text { nd } \\
\text { nd } \\
20 \\
\end{array}$ & $\begin{array}{l}- \\
- \\
- \\
- \\
- \\
- \\
- \\
- \\
- \\
- \\
- \\
- \\
-\end{array}$ \\
\hline \multicolumn{6}{|c|}{ Beta/gamma emitters ( $( \pm 10 \%)$} \\
\hline $\begin{array}{l}\text { Gross beta } \\
\frac{14}{14} \mathrm{C} \\
{ }^{60} \mathrm{Co} \\
{ }^{90} \mathrm{Sr} \rho 0 \mathrm{Y} \\
{ }^{99} \mathrm{Tc} \\
{ }^{134} \mathrm{Cs} \\
{ }^{137} \mathrm{Cs} /{ }^{137 m} \mathrm{Ba} \\
{ }^{152} \mathrm{Eu} \\
{ }^{154} \mathrm{Eu} \\
{ }^{155} \mathrm{Eu} \\
{ }^{241} \mathrm{Pu}\end{array}$ & $\begin{array}{l}(\mathrm{Bq} / \mathrm{g}) \\
(\mathrm{Bq} / \mathrm{g}) \\
(\mathrm{Bq} / \mathrm{g}) \\
(\mathrm{Bq} / \mathrm{g}) \\
(\mathrm{Bq} / \mathrm{g}) \\
(\mathrm{Bq} / \mathrm{g}) \\
(\mathrm{Bq} / \mathrm{g}) \\
(\mathrm{Bq} / \mathrm{g}) \\
(\mathrm{Bq} / \mathrm{g}) \\
(\mathrm{Bq} / \mathrm{g}) \\
(\mathrm{Bq} / \mathrm{g})\end{array}$ & $\begin{array}{r}4.1 e+07 \\
- \\
1.6 e+05 \\
1.6 e+07 \\
2.8 e+01 \\
<5.9 e+02 \\
3.4 e+05 \\
1.2 e+05 \\
7.5 e+04 \\
1.1 e+04 \\
2.9 e+04\end{array}$ & $\begin{array}{c}3.3 e+07 \\
- \\
1.9 e+04 \\
1.2 e+07 \\
- \\
<1.3 e+03 \\
2.9 e+05 \\
3.4 e+04 \\
2.2 e+04 \\
<7.2 e+03 \\
-\end{array}$ & $\begin{array}{r}4.0 e+07 \\
- \\
3.4 e+04 \\
1.4 e+07 \\
-\overline{1} \\
<1.5 e+03 \\
2.9 e+05 \\
4.0 e+04 \\
2.1 e+04 \\
<8.1 e+03 \\
-\end{array}$ & $\begin{array}{l}3.6 e+07 \\
5.1 e+02 \\
6.0 e+04 \\
2.2 e+07 \\
- \\
- \\
4.5 e+05 \\
5.2 e+04 \\
4.4 e+04 \\
7.0 e+03 \\
-\end{array}$ \\
\hline
\end{tabular}




\begin{tabular}{|c|c|c|c|c|c|}
\hline $\begin{array}{l}\text { Characteristic } \\
\text { (Analysis) }\end{array}$ & & 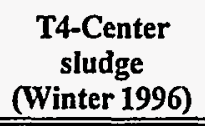 & $\begin{array}{c}\text { T4-North } \\
\text { N4 sludge } \\
\text { (Winter 1997) }\end{array}$ & $\begin{array}{c}\text { T4-South } \\
\text { S4 sludge } \\
\text { (Winter 1997) } \\
\end{array}$ & 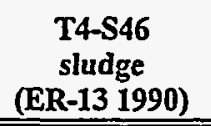 \\
\hline \multicolumn{6}{|c|}{ Alpha emitters $( \pm 10 \%)$} \\
\hline $\begin{array}{l}\text { Gross alpha } \\
{ }^{232} \mathrm{Th} \\
{ }^{233} \mathrm{U} \cdot \\
{ }^{234} \mathrm{U} \\
{ }^{233} \mathrm{U} \\
{ }^{238} \mathrm{U} \\
{ }^{237} \mathrm{~Np} \\
{ }^{241} \mathrm{Am} \\
{ }^{244} \mathrm{Cm} \\
{ }^{252} \mathrm{Cf} \\
{ }^{\text {Total } \mathrm{Pu} \text { alpha }} \\
{ }^{238} \mathrm{Pu} \\
{ }^{239} \mathrm{Pu} /{ }^{240} \mathrm{Pu}\end{array}$ & $\begin{array}{l}(\mathrm{Bq} / \mathrm{g}) \\
(\mathrm{Bq} / \mathrm{g}) \\
(\mathrm{Bq} / \mathrm{g}) \\
(\mathrm{Bq} / \mathrm{g}) \\
(\mathrm{Bq} / \mathrm{g}) \\
(\mathrm{Bq} / \mathrm{g}) \\
(\mathrm{Bq} / \mathrm{g}) \\
(\mathrm{Bq} / \mathrm{g}) \\
(\mathrm{Bq} / \mathrm{g}) \\
(\mathrm{Bq} / \mathrm{g}) \\
(\mathrm{Bq} / \mathrm{g}) \\
(\mathrm{Bq} / \mathrm{g}) \\
(\mathrm{Bq} / \mathrm{g}) \\
(\mathrm{Bq} / \mathrm{g})\end{array}$ & $\begin{array}{l}6.0 e+05 \\
5.0 e+02 \\
2.4 \mathrm{e}+04 \\
1.8 \mathrm{e}+02 \\
2.1 \mathrm{e}+00 \\
9.7 \mathrm{e}+01 \\
1.9 \mathrm{e}+01 \\
8.0 \mathrm{e}+03 \\
5.3 e+05 \\
- \\
3.5 \mathrm{e}+04 \\
2.2 \mathrm{e}+04 \\
1.3 \mathrm{e}+04 \\
-\end{array}$ & $\begin{array}{l}3.0 \mathrm{e}+05 \\
1.6 \mathrm{e}+02 \\
5.4 \mathrm{e}+03 \\
7.1 \mathrm{e}+01 \\
1.1 \mathrm{e}+00 \\
3.9 \mathrm{e}+01 \\
- \\
1.6 \mathrm{e}+04 \\
2.5 \mathrm{e}+05 \\
- \\
3.2 \mathrm{e}+04 \\
2.7 \mathrm{e}+04 \\
5.2 \mathrm{e}+03 \\
-\end{array}$ & $\begin{array}{l}3.6 e+05 \\
1.8 e+02 \\
6.9 e+03 \\
9.6 e+01 \\
1.2 e+00 \\
4.0 e+01 \\
- \\
2.3 e+04 \\
2.9 e+05 \\
- \\
3.3 e+04 \\
2.7 e+04 \\
6.5 e+03 \\
-\end{array}$ & $\begin{array}{l}3.7 e+05 \\
5.8 e+02 \\
7.1 e+03 \\
- \\
- \\
- \\
- \\
8.3 e+03 \\
2.1 e+05 \\
\\
- \\
2.2 e+04 \\
4.6 e+03 \\
-\end{array}$ \\
\hline \multicolumn{6}{|c|}{ Uranium isotopics by TIMS $( \pm 0.5 \%)$} \\
\hline $\begin{array}{l}{ }^{233} U \\
{ }^{234} \mathrm{U} \\
{ }^{235} \mathrm{U} \\
{ }^{236} \mathrm{U} \\
{ }^{238} \mathrm{U} /{ }^{235} \mathrm{U} \text { FEM } \\
{ }^{233} \mathrm{U} \\
{ }^{233} \mathrm{U}\end{array}$ & $\begin{array}{l}\text { (atom \%) } \\
\text { (atom \%) } \\
\text { (atom \%) } \\
\text { (atom \%) } \\
\text { (atom \%) } \\
\\
(\mathrm{mg} / \mathrm{Kg}) \\
(\mathrm{mg} / \mathrm{Kg})\end{array}$ & $\begin{array}{r}0.89 \\
0.01 \\
0.38 \\
0.01 \\
98.71 \\
\\
64 \\
69 \\
30 \\
\end{array}$ & $\begin{array}{r}0.498 \\
0.010 \\
0.479 \\
0.007 \\
99.006 \\
\\
88 \\
\\
15 \\
15 \\
\end{array}$ & $\begin{array}{r}0.605 \\
0.013 \\
0.516 \\
0.008 \\
98.858 \\
76 \\
19 \\
17\end{array}$ & $\begin{array}{l}- \\
- \\
- \\
- \\
- \\
- \\
- \\
-\end{array}$ \\
\hline \multicolumn{6}{|c|}{ Uranium isotopics by ICP-MS ( $\pm 2 \%)$} \\
\hline $\begin{array}{l}{ }^{233} \mathrm{U} \\
{ }^{234} \mathrm{U} \\
{ }^{235} \mathrm{U} \\
{ }^{236} \mathrm{U} \\
{ }^{238} \mathrm{U} \\
{ }^{238} \mathrm{U} /{ }^{235} \mathrm{UFEM}^{\mathrm{h}} \\
{ }^{233} \mathrm{U} \\
{ }^{235} \mathrm{U}\end{array}$ & $\begin{array}{l}\text { (atom \%) } \\
\text { (atom \%) } \\
\text { (atom \%) } \\
\text { (atom \%) } \\
\text { (atom \%) }\end{array}$ & $\begin{array}{l}- \\
- \\
- \\
- \\
- \\
-\end{array}$ & $\begin{array}{l}0.501 \\
0.011 \\
0.478 \\
0.008 \\
99.002 \\
87 \\
16 \\
15\end{array}$ & $\begin{array}{l}0.613 \\
0.012 \\
0.517 \\
0.008 \\
98.851 \\
75 \\
\\
21 \\
18\end{array}$ & $\begin{array}{l}- \\
- \\
- \\
- \\
- \\
-\end{array}$ \\
\hline
\end{tabular}




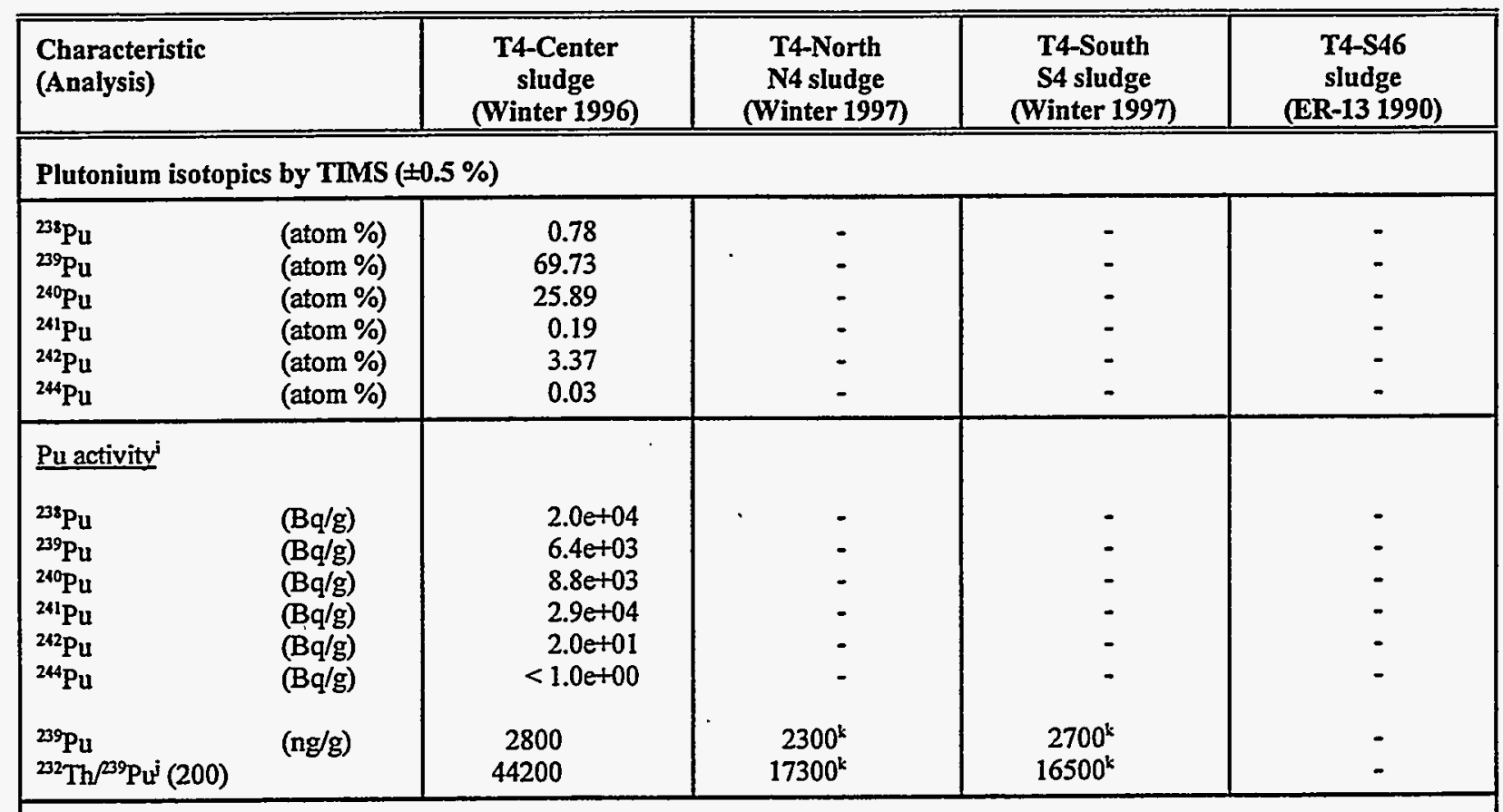

(a)\% Water content, (b) Total inorganic carbon, (c) Total carbon, (d) Total organic carbon, (e) Nitric-hydrochloric acid preparation for this element, (f) RCRA regulatory limits; $\mathrm{Ni}$ and $\mathrm{Il}$ limits are proposed,(g) Nitric-hydrofluoric acid preparation for this element, (h) Denature ratios for uranium, ${ }^{238} U /{ }^{335} U$ FEM, (i) Calculated from mass spec. and total Pu alpha data, (j) Denature ratio for plutonium, ${ }^{232} \mathrm{Th} /{ }^{239} \mathrm{Pu},(\mathrm{k})$ Based on alpha data, assumes ${ }^{239} \mathrm{Pu}={ }^{239} \mathrm{Pu}+{ }^{240} \mathrm{Pu}$ as a conservative estimate. 
Table 12 Analytical data for OHF tank T9 sludge

\begin{tabular}{|c|c|c|c|c|c|c|}
\hline \multicolumn{3}{|c|}{$\begin{array}{l}\text { Characteristic } \\
\text { (Analysis) }\end{array}$} & $\begin{array}{c}\begin{array}{c}\text { T9-Center } \\
\text { sludge } \\
\text { (Winter 1996) }\end{array} \\
\end{array}$ & $\begin{array}{c}\text { T9-North } \\
\text { N9 sludge } \\
\text { (Winter 1997) } \\
\end{array}$ & $\begin{array}{c}\text { T9-South } \\
\text { S9 sludge } \\
\text { (Winter 1997) } \\
\end{array}$ & $\begin{array}{c}\text { T9-S48 } \\
\text { sludge } \\
\text { (ER-13 1990) } \\
\end{array}$ \\
\hline \multicolumn{7}{|c|}{ Physical properties and miscellaneous data } \\
\hline \multicolumn{3}{|c|}{$\begin{array}{l}\text { Request number } \\
\text { Sample number } \\
\text { pH (water wash) }\end{array}$} & $\begin{array}{r}7618 \mathrm{~A} \\
960221-117 \\
9.3\end{array}$ & $\begin{array}{r}7973 \mathrm{~B} \\
970128-019 \\
9.3\end{array}$ & $\begin{array}{r}7973 B \\
970128-018 \\
9.1\end{array}$ & - \\
\hline \multicolumn{2}{|c|}{$\begin{array}{l}\text { Water } \\
\text { Bulk density } \\
\text { TIC } \\
\text { TC }^{\mathrm{c}} \\
\text { TOC }^{\mathrm{d}}\end{array}$} & $\begin{array}{l}(\%) \\
(\mathrm{g} / \mathrm{mL}) \\
(\mathrm{mg} / \mathrm{Kg}) \\
(\mathrm{mg} / \mathrm{Kg}) \\
(\mathrm{mg} / \mathrm{Kg})\end{array}$ & $\begin{array}{c}70.2 \\
1.16 \\
16000 \\
16000 \\
<100\end{array}$ & $\begin{array}{c}72.6 \\
1.34 \\
5900 \\
11400 \\
5500\end{array}$ & $\begin{array}{c}64.8 \\
1.58 \\
9700 \\
28200 \\
18500\end{array}$ & $\begin{array}{c}65.7 \\
- \\
- \\
7620\end{array}$ \\
\hline \multicolumn{7}{|c|}{ RCRA Metals ( $\pm 10 \%$ ) } \\
\hline & $\begin{array}{l}(100)^{t} \\
(100) \\
(2000) \\
(20) \\
(100) \\
(4) \\
(1000) \\
(100) \\
(20) \\
(18)\end{array}$ & $\begin{array}{l}(\mathrm{mg} / \mathrm{Kg}) \\
(\mathrm{mg} / \mathrm{Kg}) \\
(\mathrm{mg} / \mathrm{Kg}) \\
(\mathrm{mg} / \mathrm{Kg}) \\
(\mathrm{mg} / \mathrm{Kg}) \\
(\mathrm{mg} / \mathrm{Kg}) \\
(\mathrm{mg} / \mathrm{Kg}) \\
(\mathrm{mg} / \mathrm{Kg}) \\
(\mathrm{mg} / \mathrm{Kg}) \\
(\mathrm{mg} / \mathrm{Kg})\end{array}$ & $\begin{array}{c}<1.2 \\
<1.4 \\
81.3 \\
10.9 \\
85.1 \\
1.80 \\
452 \\
521 \\
<1.4 \\
<1.4\end{array}$ & $\begin{array}{c}<1.9 \\
- \\
29.1 \\
14.6 \\
128 \\
- \\
118 \\
- \\
- \\
-\end{array}$ & $\begin{array}{c}<2.0 \\
- \\
63.6 \\
9.61 \\
91.2 \\
- \\
158 \\
- \\
- \\
-\end{array}$ & $\begin{array}{l}\quad 0.21 \\
<2 \\
115 \\
7.8 \\
<10 \\
39 \\
380 \\
540 \\
<2 \\
<2\end{array}$ \\
\hline \multicolumn{7}{|c|}{ Process metals $( \pm 10 \%)$} \\
\hline $\begin{array}{l}\mathrm{Al} \\
\mathrm{B} \\
\mathrm{Be} \\
\mathrm{Ca} \\
\mathrm{Co} \\
\mathrm{Cu} \\
\mathrm{Cs} \\
\mathrm{Fe} \\
\mathrm{K} \\
\mathrm{Mg} \\
\mathrm{Mn} \\
\mathrm{Na} \\
\mathrm{P} \\
\mathrm{Sb} \\
\mathrm{Si}^{\mathrm{g}} \\
\mathrm{Sr} \\
\mathrm{Th} \\
\mathrm{U} \\
\mathrm{V} \\
\mathrm{Zn}\end{array}$ & & $\begin{array}{l}(\mathrm{mg} / \mathrm{Kg}) \\
(\mathrm{mg} / \mathrm{Kg}) \\
(\mathrm{mg} / \mathrm{Kg}) \\
(\mathrm{mg} / \mathrm{Kg}) \\
(\mathrm{mg} / \mathrm{Kg}) \\
(\mathrm{mg} / \mathrm{Kg}) \\
(\mathrm{mg} / \mathrm{Kg}) \\
(\mathrm{mg} / \mathrm{Kg}) \\
(\mathrm{mg} / \mathrm{Kg}) \\
(\mathrm{mg} / \mathrm{Kg}) \\
(\mathrm{mg} / \mathrm{Kg}) \\
(\mathrm{mg} / \mathrm{Kg}) \\
(\mathrm{mg} / \mathrm{Kg}) \\
(\mathrm{mg} / \mathrm{Kg}) \\
(\mathrm{mg} / \mathrm{Kg}) \\
(\mathrm{mg} / \mathrm{Kg}) \\
(\mathrm{mg} / \mathrm{Kg}) \\
(\mathrm{mg} / \mathrm{Kg}) \\
(\mathrm{mg} / \mathrm{Kg}) \\
(\mathrm{mg} / \mathrm{Kg})\end{array}$ & $\begin{array}{c}34500 \\
41.6 \\
45.4 \\
32800 \\
9.54 \\
117 \\
1.43 \\
17900 \\
974 \\
5140 \\
337 \\
6640 \\
5750 \\
<20 \\
3640 \\
908 \\
56800 \\
2510 \\
<7.8 \\
149\end{array}$ & $\begin{array}{c}4110 \\
28.4 \\
19.6 \\
28900 \\
55.7 \\
80.6 \\
- \\
21900 \\
826 \\
1520 \\
327 \\
5940 \\
4140 \\
<19 \\
- \\
751 \\
76800 \\
2700 \\
7.02 \\
162 \\
\end{array}$ & $\begin{array}{c}14900 \\
24.3 \\
48.9 \\
41100 \\
27.3 \\
95.0 \\
- \\
7740 \\
769 \\
2190 \\
252 \\
5280 \\
2130 \\
<20 \\
- \\
2400 \\
54800 \\
2220 \\
5.60 \\
144 \\
\end{array}$ & $\begin{array}{r}- \\
- \\
- \\
- \\
- \\
- \\
- \\
- \\
- \\
- \\
- \\
- \\
- \\
- \\
\end{array}$ \\
\hline
\end{tabular}




\begin{tabular}{|c|c|c|c|c|c|}
\hline $\begin{array}{l}\text { Characteristic } \\
\text { (Analysis) }\end{array}$ & & $\begin{array}{c}\begin{array}{c}\text { T9-Center } \\
\text { sludge } \\
\text { (Winter 1996) }\end{array} \\
\end{array}$ & $\begin{array}{c}\text { T9-North } \\
\text { N9 sludge } \\
\text { (Winter 1997) } \\
\end{array}$ & $\begin{array}{c}\text { T9-South } \\
\text { S9 sludge } \\
\text { (Winter 1997) } \\
\end{array}$ & $\begin{array}{c}\text { T9-S48 } \\
\text { sludge } \\
\text { (ER-13 1990) } \\
\end{array}$ \\
\hline \multicolumn{6}{|c|}{ Semi-quantitative elements by ICP-MS $( \pm 30-50 \%)$} \\
\hline $\begin{array}{l}\mathrm{Bi} \\
\mathrm{Ga} \\
\mathrm{I} \\
\mathrm{Li} \\
\mathrm{Te} \\
\mathrm{Zr} \\
\mathrm{Lanthanides} \\
\text { (57) } \mathrm{La} \\
(58) \mathrm{Ce} \\
(59) \mathrm{Pr} \\
(60) \mathrm{Nd} \\
(62) \mathrm{Sm} \\
\text { (63) } \mathrm{Eu}\end{array}$ & $\begin{array}{c}\text { (mg/Kg) } \\
(\mathrm{mg} / \mathrm{Kg}) \\
(\mathrm{mg} / \mathrm{Kg}) \\
(\mathrm{mg} / \mathrm{Kg}) \\
(\mathrm{mg} / \mathrm{Kg}) \\
(\mathrm{mg} / \mathrm{Kg}) \\
\\
(\mathrm{mg} / \mathrm{Kg}) \\
(\mathrm{mg} / \mathrm{Kg}) \\
(\mathrm{mg} / \mathrm{Kg}) \\
(\mathrm{mg} / \mathrm{Kg}) \\
(\mathrm{mg} / \mathrm{Kg}) \\
(\mathrm{mg} / \mathrm{Kg})\end{array}$ & $\begin{array}{l}: \\
: \\
: \\
- \\
- \\
- \\
-\end{array}$ & $\begin{array}{c}14 \\
3.4 \\
21 \\
320 \\
160 \\
35 \\
\\
7.9 \\
8.6 \\
1.2 \\
6.3 \\
2.1 \\
0.54\end{array}$ & $\begin{array}{c}22 \\
6.6 \\
73 \\
1200 \\
70 \\
38 \\
\\
8.9 \\
17 \\
1.3 \\
5.4 \\
2.5 \\
0.92\end{array}$ & $\begin{array}{l}- \\
: \\
: \\
- \\
- \\
- \\
- \\
-\end{array}$ \\
\hline \multicolumn{6}{|c|}{ Anions on water wash by ion chromatography $( \pm 10 \%)$} \\
\hline $\begin{array}{l}\text { Inorganic } \\
\text { Bromide } \\
\text { Chloride } \\
\text { Chromate } \\
\text { Fluoride } \\
\text { Nitrate } \\
\text { Nitrite } \\
\text { Phosphate } \\
\text { Sulphate } \\
\text { Organic } \\
\text { Acetate } \\
\text { Citrate } \\
\text { Formate } \\
\text { Oxalate } \\
\text { Phthalate } \\
\\
\text { Organic TICs } \\
\text { cis-Aconitate } \\
\text { trans-Aconitate } \\
\text { iso-Citrate } \\
\text { Malonate } \\
\text { Propionate }\end{array}$ & $\begin{array}{l}(\mathrm{mg} / \mathrm{Kg}) \\
(\mathrm{mg} / \mathrm{Kg}) \\
(\mathrm{mg} / \mathrm{Kg}) \\
(\mathrm{mg} / \mathrm{Kg}) \\
(\mathrm{mg} / \mathrm{Kg}) \\
(\mathrm{mg} / \mathrm{Kg}) \\
(\mathrm{mg} / \mathrm{Kg}) \\
(\mathrm{mg} / \mathrm{Kg}) \\
\\
(\mathrm{mg} / \mathrm{Kg}) \\
(\mathrm{mg} / \mathrm{Kg}) \\
(\mathrm{mg} / \mathrm{Kg}) \\
(\mathrm{mg} / \mathrm{Kg}) \\
(\mathrm{mg} / \mathrm{Kg}) \\
\\
(\mathrm{mg} / \mathrm{Kg}) \\
(\mathrm{mg} / \mathrm{Kg}) \\
(\mathrm{mg} / \mathrm{Kg}) \\
(\mathrm{mg} / \mathrm{Kg}) \\
(\mathrm{mg} / \mathrm{Kg})\end{array}$ & $\begin{array}{r}70 \\
3760 \\
- \\
140 \\
869 \\
219 \\
<195 \\
616\end{array}$ & $\begin{array}{l}32.7 \\
3700 \\
<10 \\
82.1 \\
845 \\
324 \\
<10 \\
558 \\
\\
\\
<5 \\
<10 \\
<5 \\
<5 \\
<10 \\
\\
\\
\\
\text { nd } \\
\text { nd } \\
\text { nd } \\
\text { nd } \\
\text { nd }\end{array}$ & $\begin{array}{l}26.1 \\
3240 \\
<10 \\
87.8 \\
748 \\
272 \\
<9 \\
503 \\
\\
\\
<5 \\
<10 \\
6.24 \\
282 \\
<10 \\
\\
\\
\text { nd } \\
\text { nd } \\
\text { nd } \\
\text { nd } \\
\text { nd }\end{array}$ & $\begin{array}{l}- \\
- \\
- \\
- \\
- \\
- \\
- \\
- \\
- \\
- \\
- \\
- \\
- \\
-\end{array}$ \\
\hline \multicolumn{6}{|c|}{ Beta/gamma emitters ( $( \pm 10 \%)$} \\
\hline $\begin{array}{l}\text { Gross beta } \\
{ }^{14} \mathrm{C} \\
{ }^{60} \mathrm{Co} \\
{ }^{90} \mathrm{Sr} \rho 0 \mathrm{Y} \\
{ }^{99} \mathrm{Tc} \\
{ }^{134} \mathrm{Cs} \\
{ }^{137} \mathrm{Cs} /{ }^{137 m} \mathrm{Ba} \\
{ }^{152} \mathrm{Eu} \\
{ }^{154} \mathrm{Eu} \\
{ }^{155} \mathrm{Eu} \\
{ }^{241} \mathrm{Pu}\end{array}$ & $\begin{array}{l}(\mathrm{Bq} / \mathrm{g}) \\
(\mathrm{Bq} / \mathrm{g}) \\
(\mathrm{Bq} / \mathrm{g}) \\
(\mathrm{Bq} / \mathrm{g}) \\
(\mathrm{Bq} / \mathrm{g}) \\
(\mathrm{Bq} / \mathrm{g}) \\
(\mathrm{Bq} / \mathrm{g}) \\
(\mathrm{Bq} / \mathrm{g}) \\
(\mathrm{Bq} / \mathrm{g}) \\
(\mathrm{Bq} / \mathrm{g}) \\
(\mathrm{Bq} / \mathrm{g})\end{array}$ & $\begin{array}{r}5.0 \mathrm{e}+07 \\
- \\
4.9 \mathrm{e}+04 \\
2.0 \mathrm{e}+07 \\
1.4 \mathrm{e}+02 \\
<4.8 \mathrm{e}+02 \\
2.6 \mathrm{e}+05 \\
4.3 \mathrm{e}+04 \\
3.1 \mathrm{e}+04 \\
6.9 \mathrm{e}+03 \\
3.0 \mathrm{e}+04\end{array}$ & $\begin{array}{r}2.6 \mathrm{e}+07 \\
- \\
8.4 \mathrm{e}+04 \\
1.0 \mathrm{e}+07 \\
- \\
<1.5 \mathrm{e}+03 \\
1.9 \mathrm{e}+05 \\
6.5 \mathrm{e}+04 \\
3.8 \mathrm{e}+04 \\
<5.1 \mathrm{e}+03 \\
-\end{array}$ & $\begin{array}{r}4.0 e+07 \\
- \\
5.9 e+04 \\
1.5 e+07 \\
- \\
<1.6 e+03 \\
2.1 e+05 \\
4.5 e+04 \\
3.3 e+04 \\
<8.2 e+03 \\
-\end{array}$ & $\begin{array}{l}1.6 e+07 \\
2.2 e+03 \\
4.3 e+04 \\
1.4 e+07 \\
- \\
- \\
4.0 e+05 \\
3.5 e+04 \\
8.9 e+03 \\
- \\
- \\
\end{array}$ \\
\hline
\end{tabular}




\begin{tabular}{|c|c|c|c|c|c|}
\hline \multicolumn{2}{|l|}{$\begin{array}{l}\text { Characteristic } \\
\text { (Analysis) }\end{array}$} & $\begin{array}{c}\begin{array}{c}\text { T9-Center } \\
\text { sludge } \\
\text { (Winter 1996) }\end{array} \\
\end{array}$ & $\begin{array}{c}\text { T9-North } \\
\text { N9 sludge } \\
\text { (Winter 1997) }\end{array}$ & $\begin{array}{c}\text { T9-South } \\
\text { S9 sludge } \\
\text { (Winter 1997) } \\
\end{array}$ & $\begin{array}{c}\text { T9-S48 } \\
\text { sludge } \\
\text { (ER-13 1990) } \\
\end{array}$ \\
\hline \multicolumn{6}{|c|}{ Alpha emitters $( \pm 10 \%)$} \\
\hline $\begin{array}{l}\text { Gross alpha } \\
{ }^{232} \mathrm{Th} \\
{ }^{233} \mathrm{U} \\
{ }^{234} \mathrm{U} \\
{ }^{235} \mathrm{U} \\
{ }^{238} \mathrm{U} \\
{ }^{237} \mathrm{~Np} \\
{ }^{211} \mathrm{Am} \\
{ }^{241} \mathrm{Cm} \\
{ }^{232} \mathrm{Cf} \\
\text { Total } \mathrm{Pu} \text { alpha } \\
{ }^{238} \mathrm{Pu} \\
{ }^{239} \mathrm{Pu} \\
{ }^{210} \mathrm{Pu}\end{array}$ & $\begin{array}{l}(\mathrm{Bq} / \mathrm{g}) \\
(\mathrm{Bq} / \mathrm{g}) \\
(\mathrm{Bq} / \mathrm{g}) \\
(\mathrm{Bq} / \mathrm{g}) \\
(\mathrm{Bq} / \mathrm{g}) \\
(\mathrm{Bq} / \mathrm{g}) \\
(\mathrm{Bq} / \mathrm{g}) \\
(\mathrm{Bq} / \mathrm{g}) \\
(\mathrm{Bq} / \mathrm{g}) \\
(\mathrm{Bq} / \mathrm{g}) \\
(\mathrm{Bq} / \mathrm{g}) \\
(\mathrm{Bq} / \mathrm{g}) \\
(\mathrm{Bq} / \mathrm{g}) \\
(\mathrm{Bq} / \mathrm{g})\end{array}$ & $\begin{array}{l}3.5 e+05 \\
2.3 e+02 \\
6.4 e+03 \\
5.7 e+01 \\
0.9 e+00 \\
3.1 e+01 \\
1.2 e+01 \\
1.3 e+04 \\
2.7 e+05 \\
<3.5 e+02 \\
5.8 e+04 \\
4.8 e+04 \\
9.2 e+03 \\
-\end{array}$ & $\begin{array}{l}3.5 e+05 \\
3.1 \mathrm{e}+02 \\
1.1 \mathrm{e}+04 \\
1.2 \mathrm{e}+02 \\
0.7 \mathrm{e}+00 \\
3.3 \mathrm{e}+01 \\
- \\
3.4 \mathrm{e}+04 \\
2.8 \mathrm{e}+05 \\
- \\
1.9 \mathrm{e}+04 \\
1.2 \mathrm{e}+04 \\
7.4 \mathrm{e}+03 \\
-\end{array}$ & $\begin{array}{l}3.2 \mathrm{e} \div 05 \\
2.2 \mathrm{e}+02 \\
7.3 \mathrm{e}+03 \\
8.6 \mathrm{e}+01 \\
0.7 \mathrm{e} \div 00 \\
2.7 \mathrm{e}+01 \\
- \\
9.0 \mathrm{e}+03 \\
2.7 \mathrm{e}+05 \\
- \\
3.1 \mathrm{e}+04 \\
2.4 \mathrm{e}+04 \\
7.4 \mathrm{e}+03 \\
-\end{array}$ & $\begin{array}{l}1.5 e+05 \\
\dot{-} \\
4.4 e+03 \\
- \\
- \\
- \\
\dot{-} \\
9.7 e+04 \\
<2.0 e+00 \\
- \\
1.0 e+04 \\
4.3 e+03 \\
-\end{array}$ \\
\hline \multicolumn{6}{|c|}{ Uranium isotopics by TIMS ( $\pm 0.5 \%)$} \\
\hline $\begin{array}{l}{ }^{233} \mathrm{U} \\
{ }^{234} \mathrm{U} \\
{ }^{235} \mathrm{U} \\
{ }^{236} \mathrm{U} \\
{ }^{238} \mathrm{U} /{ }^{235} \mathrm{U} F E M^{\mathrm{b}} \\
{ }^{233} \mathrm{U} \\
{ }^{233} \mathrm{U}\end{array}$ & $\begin{array}{l}\text { (atom \%) } \\
\text { (atom \%) } \\
\text { (atom \%) } \\
\text { (atom \%) } \\
\text { (atom \%) } \\
\\
(\mathrm{mg} / \mathrm{Kg} \text { ) } \\
\text { (mg/Kg) }\end{array}$ & $\begin{array}{l}0.73 \\
0.01 \\
0.50 \\
0.01 \\
98.75 \\
68 \\
\\
18 \\
12\end{array}$ & $\begin{array}{r}1.198 \\
0.019 \\
0.377 \\
0.007 \\
98.399 \\
\\
50 \\
\\
32 \\
10\end{array}$ & $\begin{array}{r}0.944 \\
0.017 \\
0.449 \\
0.007 \\
98.583 \\
\\
58 \\
\\
21 \\
9.8\end{array}$ & $\begin{array}{l}- \\
- \\
- \\
- \\
- \\
- \\
-\end{array}$ \\
\hline \multicolumn{6}{|c|}{ Uranium isotopics by ICP-MS $(\div 2 \%)$} \\
\hline $\begin{array}{l}{ }^{233} \mathrm{U} \\
{ }^{234} \mathrm{U} \\
{ }^{235} \mathrm{U} \\
{ }^{236} \mathrm{U} \\
{ }^{238} \mathrm{U} \\
{ }^{238} \mathrm{U} /{ }^{235} \mathrm{U} F E M^{\mathrm{h}} \\
{ }^{233} \mathrm{U} \\
{ }^{235} \mathrm{U}\end{array}$ & $\begin{array}{l}(\text { atom \%) } \\
\text { (atom \%) } \\
\text { (atom \%) } \\
(\text { atom \%) } \\
\text { (atom \%) }\end{array}$ & $\begin{array}{l}- \\
- \\
- \\
- \\
- \\
- \\
-\end{array}$ & $\begin{array}{r}1.192 \\
0.019 \\
0.374 \\
0.007 \\
98.408 \\
\\
51 \\
31 \\
9.8\end{array}$ & $\begin{array}{r}0.941 \\
0.015 \\
0.445 \\
0.008 \\
98.590 \\
59 \\
\\
21 \\
10\end{array}$ & $\begin{array}{l}- \\
- \\
- \\
- \\
- \\
-\end{array}$ \\
\hline
\end{tabular}




\begin{tabular}{|c|c|c|c|c|c|}
\hline $\begin{array}{l}\text { Characteristic } \\
\text { (Analysis) }\end{array}$ & & $\begin{array}{c}\text { T9-Center } \\
\text { sludge } \\
\text { (Winter 1996) }\end{array}$ & $\begin{array}{c}\text { T9-North } \\
\text { N9 sludge } \\
\text { (Winter 1997) } \\
\end{array}$ & $\begin{array}{c}\text { T9-South } \\
\text { S9 sludge } \\
\text { (Winter 1997) }\end{array}$ & $\begin{array}{c}\text { T9-S48 } \\
\text { sludge } \\
\text { (ER-13 1990) } \\
\end{array}$ \\
\hline \multicolumn{6}{|c|}{ Plutonium isotopics by TIMS ( $\pm 0.5 \%)$} \\
\hline $\begin{array}{l}{ }^{238} \mathrm{Pu} \\
{ }^{239} \mathrm{Pu} \\
{ }^{240} \mathrm{Pu} \\
{ }^{241} \mathrm{Pu} \\
{ }^{24} \mathrm{Pu} \\
{ }^{44} \mathrm{Pu}\end{array}$ & $\begin{array}{l}\text { (atom \%) } \\
\text { (atom \%) } \\
\text { (atom \%) } \\
\text { (atom \%) } \\
\text { (atom \%) } \\
\text { (atom \%) }\end{array}$ & $\begin{array}{r}2.45 \\
77.60 \\
17.04 \\
0.25 \\
2.66 \\
<0.01\end{array}$ & $\begin{array}{l}- \\
- \\
-\end{array}$ & $\begin{array}{l}- \\
- \\
- \\
-\end{array}$ & $\begin{array}{l}- \\
- \\
- \\
-\end{array}$ \\
\hline \multicolumn{6}{|l|}{ Pu activity } \\
\hline $\begin{array}{l}{ }^{238} \mathrm{Pu} \\
{ }^{209} \mathrm{Pu} \\
{ }^{240} \mathrm{Pu} \\
{ }^{241} \mathrm{Pu} \\
{ }^{242} \mathrm{Pu} \\
{ }^{244} \mathrm{Pu}\end{array}$ & $\begin{array}{l}(\mathrm{Bq} / \mathrm{g}) \\
(\mathrm{Bq} / \mathrm{g}) \\
(\mathrm{Bq} / \mathrm{g}) \\
(\mathrm{Bq} / \mathrm{g}) \\
(\mathrm{Bq} / \mathrm{g}) \\
(\mathrm{Bq} / \mathrm{g})\end{array}$ & $\begin{array}{r}4.8 \mathrm{e}+04 \\
5.5 \mathrm{e}+03 \\
4.5 \mathrm{e}+03 \\
3.0 \mathrm{e}+04 \\
1.2 \mathrm{e}+01 \\
<1.0 \mathrm{e}+00\end{array}$ & - & $\begin{array}{l}- \\
- \\
- \\
-\end{array}$ & $\begin{array}{l}- \\
- \\
- \\
-\end{array}$ \\
\hline 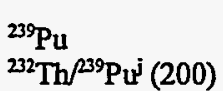 & (ng/g) & $\begin{array}{r}2410 \\
23600\end{array}$ & $\begin{array}{r}3200^{k} \\
23800^{k}\end{array}$ & $\begin{array}{r}3200^{k} \\
17000^{k}\end{array}$ & - \\
\hline
\end{tabular}




\subsection{Discussion of OHF Supernatant Characteristics}

The analytical data for the OHF supernatant samples (Table 3 - 7) are based on samples that were first clarified by centrifugation and then stabilized with nitric acid. Extra care was taken to remove suspended particles from the liquid samples because the particulate material can be an artifact of the sampling technique and could bias the liquid phase data. Characteristic of most high $\mathrm{pH}$ solutions the thorium, uranium and other actinide elements in the ORNL waste tanks form an insoluble hydroxide or oxide precipitate. This chemical behavior is usually apparent with the supernatant when the $\mathrm{pH}$ is compared to the uranium concentration and the alpha activity.

The Group IA elements, sodium and potassium, are very soluble in the supernatant at any $\mathrm{pH}$. In general, the concentration of Group IIA metals such as calcium and strontium will increase in the supernatant as the $\mathrm{pH}$ decreases. These Group IIA metals are initially soluble in the liquid phase at high $\mathrm{pH}$, but as carbon dioxide is absorbed into the supernatant from the air, both calcium and strontium form insoluble carbonate compounds. The general distribution of radioactivity in the waste tanks is a function of the $\mathrm{pH}$, where at higher $\mathrm{pH}$ the ${ }^{137} \mathrm{Cs}$ dominates the beta activity in the liquid phase, and the ${ }^{90} \mathrm{Sr} /{ }^{90} \mathrm{Y}$ is the predominate source of the beta activity in the sludge phase. At high $\mathrm{pH}$, the actinide elements are mostly insoluble which corresponds to most of the alpha activity being concentrated in the sludge phase.

As expected, the concentration of silicon compounds increases in the supernatant as the $\mathrm{pH}$ increases. Many of the other common metals found in the waste, such as iron and magnesium, are less soluble as the $\mathrm{pH}$ increases. In general, as the $\mathrm{pH}$ decreases, the total dissolved solids in the supernatant increases. The distribution of major cations and anions in the OHF liquid samples collected from the center of each tank are illustrated in Fig. 1 and 2. The distribution illustrated in Fig. 2 is similar to Fig. 1, but with the sodium and nitrate removed to expand the scale and show more detail for species present at lower concentrations. 
Figure 1 Distribution of Major Cations and Anions in OHF Supernatant

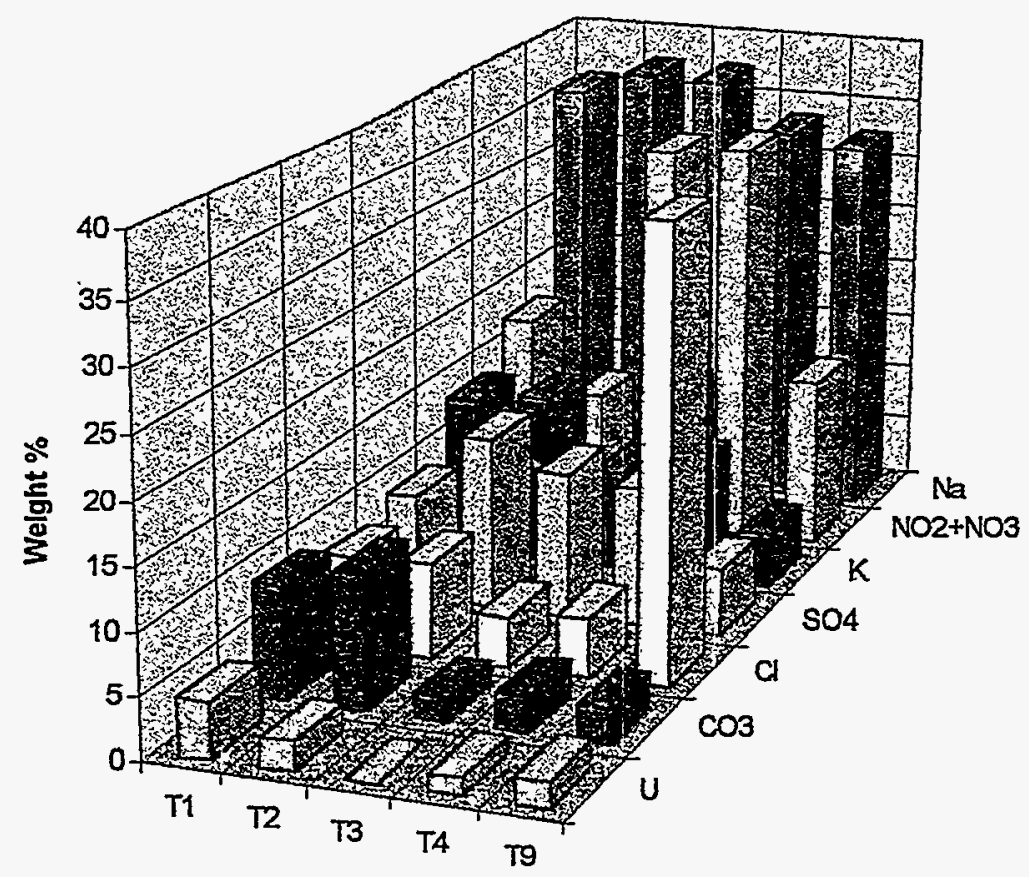

Figure 2 Distribution of Nitrate/Nitrite Salts in OHF Supernatant

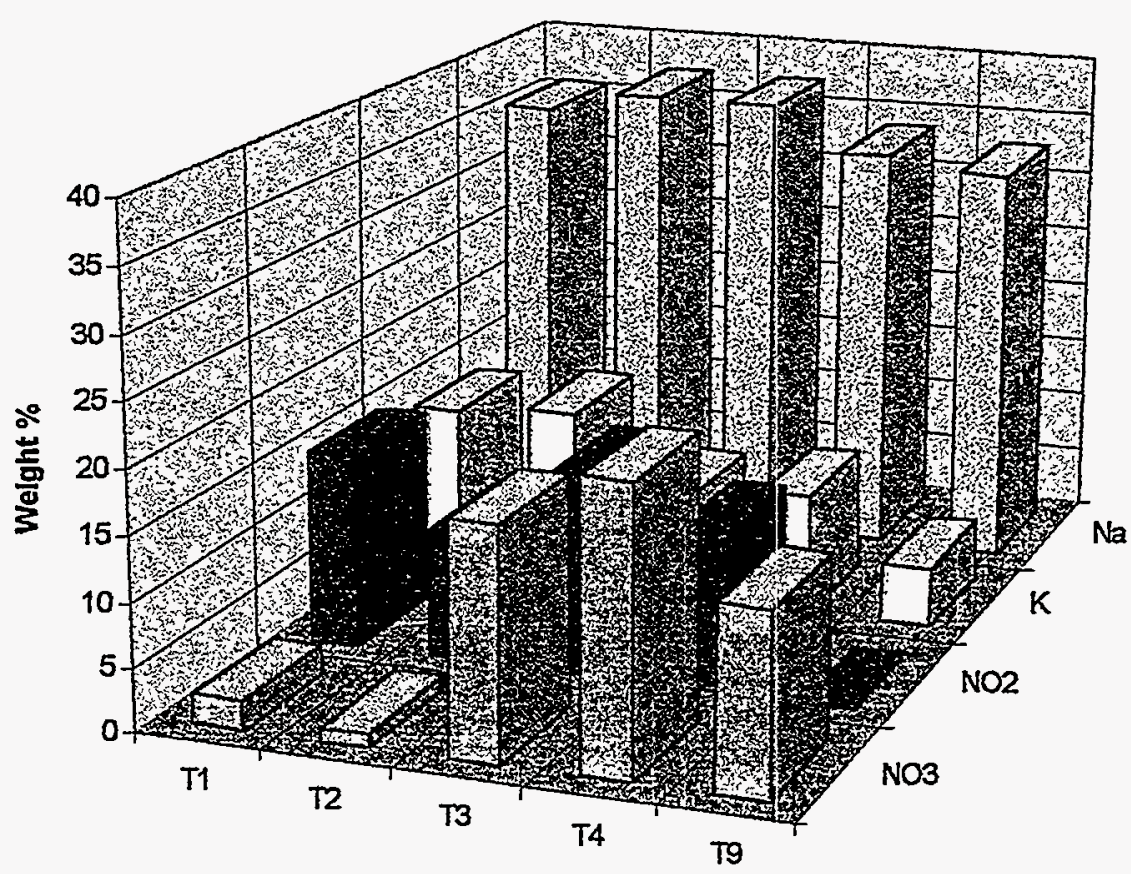


The sludge layers in the ORNL waste tanks are typically high in several RCRA metals, including chromium, mercury, and lead. Generally, these RCRA metals are below the hazard limits in a high $\mathrm{pH}$ supernatant, but as the $\mathrm{pH}$ decreases the concentration of these RCRA metals can increase to the point where the regulatory limits may be exceeded in the liquid phase.

The mass and charge balance data for the OHF supernatant samples provide a good indication of data completeness and are summarized in Table 13. The mass balance checks are based on the summation of cation and anion concentrations divided by the total solids concentration. The total solids concentration is measured directly by weighing a known volume of sample that has been dried to a constant weight. In general, the charge balance checks are less accurate than the mass balance results because one must make an assumptions about the chemical form and oxidation state for each species present in solution. The charge balance data is based on the summation of the molar cation charge divided by the summation of the molar anion charge.

Table 13 Summary of quality checks for OHF supernatant data

\begin{tabular}{|c|c|c|c|c|c|c|c|}
\hline & ank & $\begin{array}{c}\text { Mass } \\
\text { Balance } \\
\left(\mathrm{TS}_{\text {ale }} / \mathrm{TS}_{\text {meass }}\right)\end{array}$ & $\begin{array}{c}\text { Charge } \\
\text { Balance } \\
\left(\mathrm{M}^{+} / \mathbf{A}^{-}\right) \\
\end{array}$ & pH & $\begin{array}{l}{ }^{137} \mathrm{Cs} \\
(\%)\end{array}$ & $\begin{array}{c}{ }^{90} \mathrm{Sr} \rho^{90} \mathrm{Y} \\
(\%)\end{array}$ & $\begin{array}{c}\text { Beta } \\
\text { Recovery } \\
(\%) \\
\end{array}$ \\
\hline \multirow[t]{3}{*}{ Tl- } & North & 0.735 & 1.385 & 8.9 & 91.4 & 8.6 & 98.7 \\
\hline & Middle & 0.724 & 1.645 & 9.3 & 91.4 & 8.6 & 95.4 \\
\hline & South & 0.706 & 1.558 & 8.9 & 90.8 & 9.2 & 99.6 \\
\hline \multirow[t]{3}{*}{ T2- } & North & 0.701 & 1.707 & 9.3 & 96.3 & 3.8 & 102.8 \\
\hline & Middle & 0.702 & 1.709 & 9.3 & 96.1 & 3.8 & 97.4 \\
\hline & South & 0.694 & 1.656 & 9.3 & 96.1 & 3.9 & 95.6 \\
\hline \multirow[t]{3}{*}{ T3- } & North & 0.870 & 1.242 & 9.5 & 99.5 & 0.4 & 99.7 \\
\hline & Middle & 0.704 & 1.610 & 11.6 & 99.7 & 0.2 & 96.7 \\
\hline & South & 0.862 & 1.156 & 9.6 & 99.4 & 0.5 & 99.8 \\
\hline \multirow[t]{3}{*}{ T4- } & North & 0.902 & 1.203 & 10.1 & 98.4 & 1.6 & 101.8 \\
\hline & Middle & 0.774 & 1.504 & 10.4 & 98.4 & 1.6 & 97.2 \\
\hline & South & 0.909 & 1.193 & 10.1 & 98.3 & 1.7 & 101.9 \\
\hline \multirow[t]{3}{*}{ T9- } & North & 0.877 & 1.080 & 8.9 & 86.4 & 13.6 & 94.7 \\
\hline & Middle & 0.920 & 1.046 & 9.1 & 84.3 & 15.7 & 106.6 \\
\hline & South & 0.918 & 1.161 & 8.9 & 85.5 & 14.5 & 95.7 \\
\hline
\end{tabular}


The beta recovery listed in Table 13 is based on the summation of the activity for the known beta emitters divided by the gross beta activity. Considering the typical analytical errors associated which radiochemical measurements, the beta recoveries listed in Table 13 are excellent. The gross beta data reported is based on a total activity measurement by liquid scintillation counting which includes contributions from the conversion and Auger electrons. To determine the beta recovery, the total activity measurement minus the alpha activity is the gross beta value that is compared to the summation of the individual radionuclides identified. Also, one must take into account the effect that analytical error for the radioactive strontium activity can have on the value of the beta recovery. Since the ${ }^{90} \mathrm{Sr}$ is in secular equilibrium with the ${ }^{90} \mathrm{Y}$, any error on the ${ }^{90} \mathrm{Sr}$ activity would effectively be counted twice when calculating the error on the beta recovery. In general, the distribution of ${ }^{137} \mathrm{Cs}$ in the liquid waste is independent of $\mathrm{pH}$, and the ${ }^{90} \mathrm{Sr}$ activity is a function of both $\mathrm{pH}$ and carbonate concentration.

If the waste tank chemistry approaches the conditions where the $\mathrm{pH}$ is high and the carbonate concentration is low, it is possible for the ${ }^{90} \mathrm{Sr}$ to remain soluble and the ${ }^{90} \mathrm{Y}$ to precipitate as the hydroxide and disrupt the secular equilibrium. It is important to understand any conditions that could disrupt this equilibrium because some radiochemical screening techniques and the interpretation of beta dose assume that the ${ }^{90} \mathrm{Y}$ activity is equal to the ${ }^{90} \mathrm{Sr}$ activity. The separation of the strontium from the yttrium is frequently observed with ${ }^{90} \mathrm{Sr}$ contaminated water moving through soil. The soluble ${ }^{90} \mathrm{Sr}$ moves with the water and the ${ }^{90} \mathrm{Y}$ is absorbed to the soil by an ion exchange process. Past practices used clay based materials as a mobilizing agent for pumping sludge. Therefore, the sludge may have an ion exchange affinity for yttrium or other radionuclides, which could interfere with the expected behavior for some radionuclides or other chemical species.

In general, the beta/gamma emitters found in the OHF supernatant represent what would be expected for fission product waste that had been aged for 5-10 years. The relative distribution of the beta activity in the OHF supernatant is summarized in Table 14. The ORNL liquid waste is normally stored at a caustic $\mathrm{pH}$ and the radioactive cesium dominates the radioactivity in the liquid phase and radioactive strontium is the dominate activity in the sludge. If the $\mathrm{pH}$ of the supernatant in tank 
drops below neutral there would be a corresponding increase in the strontium, uranium, and many other caustic insoluble metals in the liquid phase.

Table 14 Distribution of beta activity in OHF supernatant

\begin{tabular}{|c|c|c|c|c|c|c|c|}
\hline \multirow{2}{*}{\multicolumn{2}{|c|}{ Tank }} & \multirow{2}{*}{$\mathbf{p H}$} & \multicolumn{4}{|c|}{ Percent of Total Beta Activity } & \multirow{2}{*}{$\underset{(m g / L)}{U}$} \\
\hline & & & $\begin{array}{l}{ }^{60} \mathrm{Co} \\
(\%)\end{array}$ & $\begin{array}{c}{ }^{90} \mathrm{Sr}{ }^{90} \mathrm{Y} \\
(\%)\end{array}$ & $\begin{array}{l}{ }^{99} \mathrm{Tc} \\
(\%)\end{array}$ & $\begin{array}{l}{ }^{137} \mathrm{Cs} \\
(\%)\end{array}$ & \\
\hline \multirow[t]{3}{*}{ T1- } & North & 8.9 & 0.00 & 8.6 & - & 91.4 & 246 \\
\hline & Middle & 9.3 & 0.03 & 8.6 & 0.02 & 91.4 & 281 \\
\hline & South & 8.9 & 0.00 & 9.2 & - & 90.8 & 254 \\
\hline \multirow[t]{3}{*}{ T2- } & North & 9.3 & 0.00 & 3.8 & - & 96.3 & 221 \\
\hline & Middle & 9.3 & 0.05 & 3.8 & 0.01 & 96.1 & 219 \\
\hline & South & 9.3 & 0.00 & 3.9 & - & 96.1 & 206 \\
\hline \multirow[t]{3}{*}{ T3- } & North & 9.5 & 0.04 & 0.4 & - & 99.5 & 7.72 \\
\hline & Middle & 11.6 & 0.05 & 0.2 & 0.01 & 99.7 & 0.39 \\
\hline & South & 9.6 & 0.07 & 0.5 & - & 99.4 & 7.74 \\
\hline \multirow[t]{3}{*}{ T4- } & North & 10.1 & 0.00 & 1.6 & - & 98.4 & 216 \\
\hline & Middle & 10.4 & 0.00 & 1.6 & 0.01 & 98.4 & 195 \\
\hline & South & 10.1 & 0.00 & 1.7 & - & 98.3 & 215 \\
\hline \multirow[t]{3}{*}{ T9- } & North & 8.9 & 0.00 & 13.6 & - & 86.4 & 269 \\
\hline & Middle & 9.1 & 0.02 & 15.7 & 0.01 & 84.3 & 303 \\
\hline & South & 8.9 & 0.00 & 14.5 & - & 85.5 & 275 \\
\hline
\end{tabular}

The alpha activity is usually low in the supernatant, as would be expected with a caustic $\mathrm{pH}$. If the hydroxide concentration in the supernatant is less than about $0.1 \underline{\mathrm{M}}$, the uranium can form a complex with the carbonate present and become more soluble. As shown in Table 14 the uranium concentration is generally low at the higher $\mathrm{pH}$, such as the middle sample from tank T3. Although there is no good explanation for the lower $\mathrm{pH}$ observed in liquid samples collected from the North and South ends of the T3 tank, the uranium concentration follows the $\mathrm{pH}$ as expected. 


\subsection{Discussion of OHF Sludge Characteristics}

Determination of the mass and charge balance for sludge samples are more problematic than the liquid samples because the assumptions concerning the chemical form and the oxidation state of the species present in the sludge are more ambiguous due to the complex precipitation chemistry. Also, many of the compounds in the sludge are mixed oxides and hydrated hydroxides which are not directly measured. The sludge is actually a compressed slurry with a high water content. The interstitial liquid is in close contact with the sludge and there are many more solubility equilibriums for the ionic species to take into account. The anion data for the sludge samples are based on the sum of the anions in the interstitial liquid and the water soluble anions that would be available to a water wash. The water wash would not account for the insoluble hydroxides, carbonates, and mixed oxides, which usually require some acid to dissolve. The insoluble species do not contribute to the charge balance, and the cation charge is not used in the calculation, as indicated in Table 15. Most of the nitrate reported for the sludge is due to the interstitial liquid. Considering these limitations, the compounds listed in Table 15 were used to estimate the mass and charge balance.

\section{Table 15 Assumption used for major compounds in OHF sludge}

\begin{tabular}{|c|c|c|c|}
\hline Cation & Chemical Form & $\begin{array}{c}\text { Cation } \\
\text { Charge Used }\end{array}$ & $\begin{array}{c}\text { Gravimetric } \\
\text { Factors }\end{array}$ \\
\hline \hline $\mathrm{Al}^{3+}$ & $\mathrm{Al}_{2} \mathrm{O}_{3}$ & 0 & 1.890 \\
$\mathrm{Ca}^{2+}$ & $\mathrm{CaCO}_{3}$ & 0 & 2.497 \\
$\mathrm{Fe}^{3+}$ & $\mathrm{Fe}_{2} \mathrm{O}_{3}$ & 0 & 1.430 \\
$\mathrm{~K}^{+}$ & $\mathrm{K}^{+} \mathrm{NO}_{3}^{-}$ & +1 & 2.586 \\
$\mathrm{Mg}^{2+}$ & $\mathrm{Mg}(\mathrm{OH})_{2}$ & 0 & 2.399 \\
$\mathrm{Mn}^{2+}$ & $\mathrm{Mn}(\mathrm{OH})_{2}$ & 0 & 1.619 \\
$\mathrm{Na}^{+}$ & $\mathrm{Na} \mathrm{NO}_{3}^{-}$ & +1 & 3.697 \\
$\mathrm{Th}^{4+}$ & $\mathrm{Th}(\mathrm{OH})_{4}$ & 0 & 1.293 \\
$\mathrm{UO}_{2}{ }^{2+}$ & $\mathrm{UO}_{2}\left((\mathrm{OH})_{2}-\mathrm{H}_{2} \mathrm{O}\right.$ & 0 & 1.353 \\
\hline
\end{tabular}


Table 16 summarizes the mass and charge balance for the OHF tank sludge samples. Considering the assumptions required for these calculations, the mass balance is within the analytical error range $( \pm 20-30 \%)$ for these sludge samples. The charge balance is heavily influenced by the assumptions concerning the chemical form and the corresponding balance checks are less reliable.

\section{Table 16 Summary of quality checks for OHF sludge data}

\begin{tabular}{|c|c|c|c|c|c|c|c|}
\hline & ank & $\begin{array}{c}\text { Mass } \\
\text { Balance } \\
\left(\mathrm{TS}_{\text {eale }} / \mathrm{TS}_{\text {meas }}\right) \\
\end{array}$ & $\begin{array}{l}\text { Charge } \\
\text { Balance } \\
\left(\mathrm{M}^{+} / \mathrm{A}^{-}\right) \\
\end{array}$ & pH & $\begin{array}{l}{ }^{137} \mathrm{Cs} \\
(\%) \\
\end{array}$ & $\begin{array}{c}{ }^{90} \mathrm{Sr}{ }^{{ }^{0}} \mathbf{Y} \\
(\%) \\
\end{array}$ & $\begin{array}{c}\text { Beta } \\
\text { Recovery } \\
(\%) \\
\end{array}$ \\
\hline \multirow[t]{3}{*}{ T1- } & North & 0.722 & 1.193 & 8.9 & 3.79 & 95.18 & 78.2 \\
\hline & Middle & 0.905 & 0.426 & 9.6 & 1.12 & 98.22 & 91.4 \\
\hline & South & 0.715 & 0.589 & 9.0 & 1.28 & 98.38 & 68.2 \\
\hline \multirow[t]{3}{*}{ T2- } & North & 0.897 & 0.872 & 9.2 & 1.96 & 97.52 & 74.2 \\
\hline & Middle & 0.793 & 0.352 & 9.6 & 1.11 & 98.09 & 84.4 \\
\hline & South & 0.801 & 0.922 & 9.3 & 1.10 & 98.51 & 70.1 \\
\hline \multirow[t]{3}{*}{ T3- } & North & 0.795 & 1.369 & 11.4 & 11.90 & 87.17 & 84.0 \\
\hline & Middle & 1.041 & 1.181 & 11.5 & 9.78 & 89.01 & 84.1 \\
\hline & South & 0.830 & 1.445 & 11.0 & 7.09 & 92.08 & 74.9 \\
\hline \multirow[t]{3}{*}{ T4- } & North & 0.796 & 1.010 & 9.8 & 1.39 & 98.22 & 74.7 \\
\hline & Middle & 1.075 & 0.755 & 10.4 & 1.21 & 97.51 & 81.2 \\
\hline & South & 0.834 & 1.048 & 9.9 & 1.19 & 98.38 & 71.8 \\
\hline \multirow[t]{3}{*}{ T9- } & North & 0.931 & 0.596 & 9.3 & 1.08 & 97.80 & 79.7 \\
\hline & Middle & 1.017 & 0.365 & 9.3 & 0.75 & 98.79 & 81.6 \\
\hline & South & 0.720 & 0.484 & 9.1 & 0.81 & 98.69 & 76.6 \\
\hline
\end{tabular}

${ }^{a}$ The low beta recovery is probably due to the effect of elevated levels of sulfate or some other unidentified interference on the radioactive strontium analysis. Funding was not available to pursue this problem.

The beta recovery results for the OHF sludge samples, listed in Table 16, are significantly lower than what has been observed with ORNL sludge samples from other waste tank systems. As discussed previously with the liquid samples, the low beta recovery is significantly influenced by the ${ }^{90} \mathrm{Sr}$ measurement. Therefore, any sample matrix problems affecting the measurement of radioactive strontium will be strongly reflected in the beta recovery. Considering the potential for propagated error, the comparison of the gross beta to the summation of the identified radionuclides was reasonable for the OHF sludge samples. 
The distribution of the major compounds (listed in Table 15) by weight percent are illustrated in Fig. 3 for each OHF sludge sample. The calcium carbonate and thorium hydroxide/oxide accounts for most of the sludge mass in the OHF sludge samples. The balance of the sludge mass is dominated by the sodium nitrate, aluminum oxide, and various magnesium and uranium compounds. An expanded view of the distribution for the total uranium and thorium concentration in each OHF sludge sample are shown in Fig. 4, which illustrates the relatively high level of thorium present in the OHF tanks. 
Figure 3 Distribution of Major Compounds in OHF Sludge

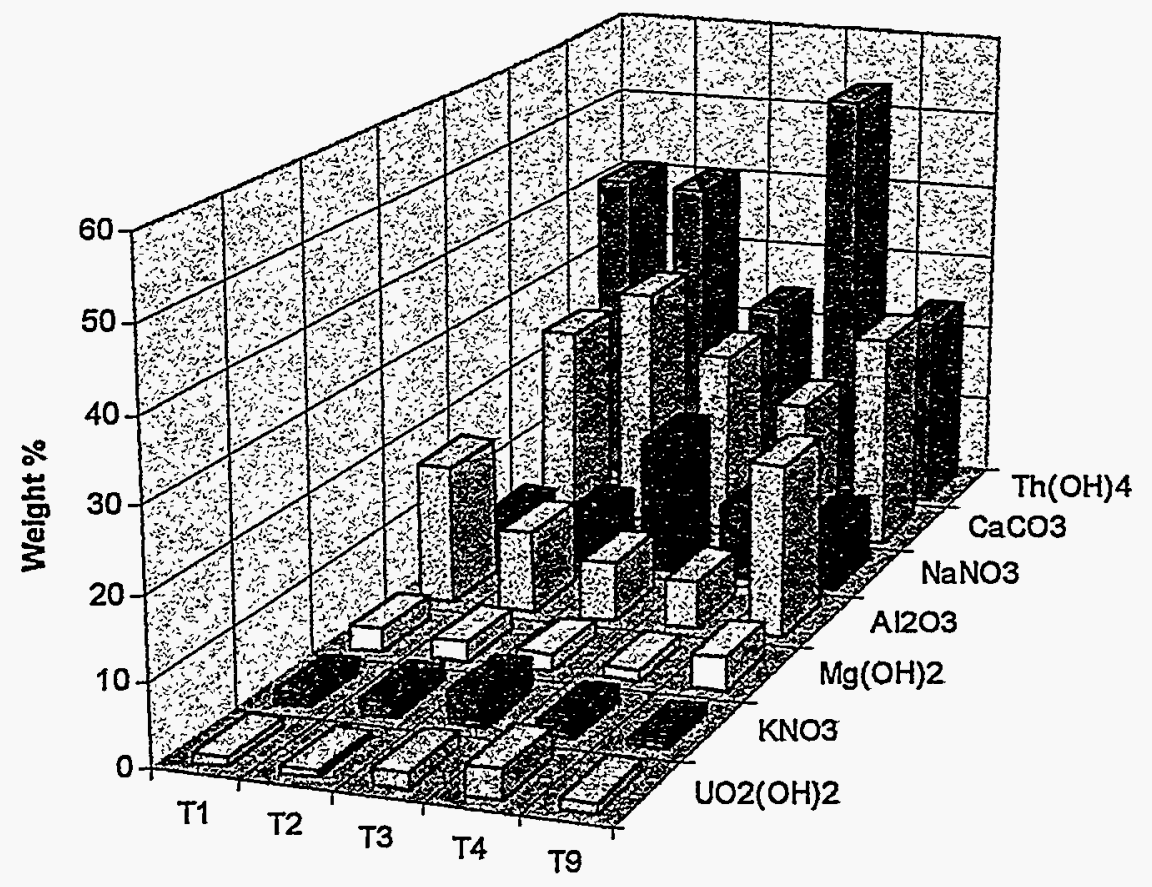

Figure 4 Distribution of Uranium and Thorium in OHF Sludge

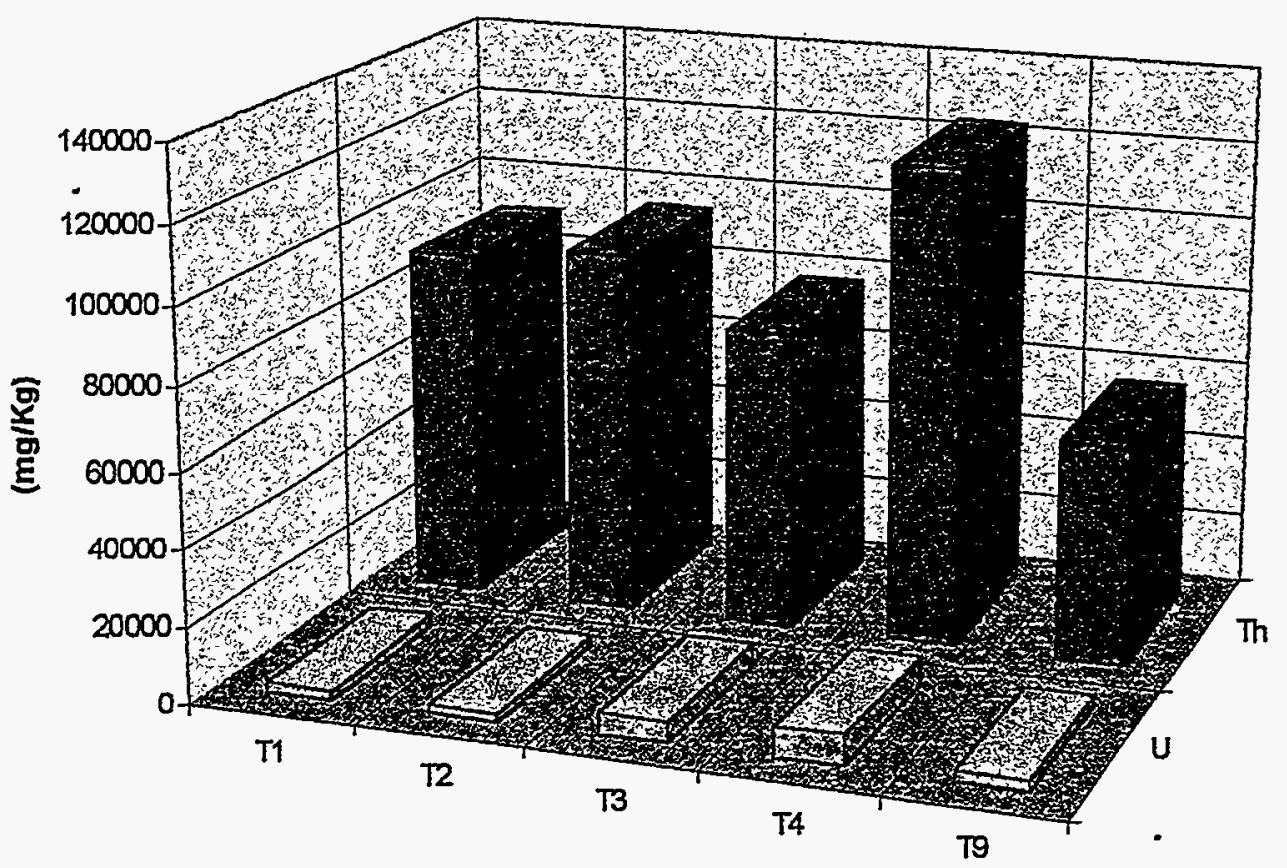


The distribution of the beta emitters found in the OHF sludge samples are summarized in Table 17. The distributions of the beta activity are shown to be dependent upon the radionuclides present, which is a function of the age of the radioactive waste, and the $\mathrm{pH}$ of the supernatant found over the sludge. Under the typical basic conditions for ORNL waste tanks, the major difference in the beta distribution between the supernatant and the sludge is that the distribution of the longer lived fission products $\left({ }^{90} \mathrm{Sr}\right.$ and $\left.{ }^{137} \mathrm{Cs}\right)$ are reversed due to the differences in solubility. The Group IA metals $\left({ }^{134} \mathrm{Cs}\right.$ and $\left.{ }^{137} \mathrm{Cs}\right)$ and the radionuclides that form anionic species $\left({ }^{99} \mathrm{TcO}_{4}^{-},{ }^{129} \mathrm{I}^{-}\right.$, and $\left.{ }^{129} \mathrm{IO}_{3}^{-}\right)$are more soluble in the supernatant at any $\mathrm{pH}$. The solubility of the Group IIA metals $\left({ }^{90} \mathrm{Sr}\right)$ in the supernatant are a function of both $\mathrm{pH}$ and carbonate concentration. At high $\mathrm{pH}$ most of the other common metals, lanthanides, and actinide elements form insoluble hydroxides and mixed oxides and are found in the sludge. If present, the ${ }^{99} \mathrm{Tc}$ activity would be higher in the supernatant than the sludge. The source of most of the ${ }^{99} \mathrm{Tc}$ found in ORNL sludge samples was the interstitial liquid, and not as insoluble forms of technetium. The shorter lived radionuclides observed include the europium $\left({ }^{152} \mathrm{Eu},{ }^{154} \mathrm{Eu}\right.$, and $\left.{ }^{155} \mathrm{Eu}\right)$ isotopes which are usually found in the sludge and to some extent ${ }^{13} \mathrm{Cs}$ which is more abundant in the liquid phase.

Table 17 Distribution of beta activity in OHF sludge

\begin{tabular}{|c|c|c|c|c|c|c|c|c|}
\hline \multirow{2}{*}{\multicolumn{2}{|c|}{ Tank }} & \multirow{2}{*}{ pH } & \multicolumn{5}{|c|}{ Percent of Total Beta Activity } & \multirow{2}{*}{$\underset{(\mathrm{mg} / \mathrm{L})}{\mathrm{U}}$} \\
\hline & & & $\begin{array}{l}{ }^{60} \mathrm{Co} \\
(\%)\end{array}$ & $\begin{array}{c}{ }^{90} \mathrm{Sr}{ }^{90} \mathrm{Y} \\
(\%) \\
\end{array}$ & $\begin{array}{l}{ }^{99} \mathrm{Tc} \\
(\%)\end{array}$ & $\begin{array}{l}{ }^{137} \mathrm{Cs} \\
(\%)\end{array}$ & $\begin{array}{c}{ }^{152-155} \mathrm{Eu} \\
(\%)\end{array}$ & \\
\hline $\mathrm{T} 1-$ & $\begin{array}{l}\text { North } \\
\text { Middle } \\
\text { South }\end{array}$ & $\begin{array}{l}8.9 \\
9.6 \\
9.0\end{array}$ & $\begin{array}{l}0.35 \\
0.16 \\
0.09\end{array}$ & $\begin{array}{l}95.18 \\
98.22 \\
98.38\end{array}$ & $<0.01$ & $\begin{array}{l}3.79 \\
1.12 \\
1.28\end{array}$ & $\begin{array}{l}0.59 \\
0.28 \\
0.18\end{array}$ & $\begin{array}{l}1410 \\
2420 \\
1360\end{array}$ \\
\hline T2- & $\begin{array}{l}\text { North } \\
\text { Middle } \\
\text { South }\end{array}$ & $\begin{array}{l}9.2 \\
9.6 \\
9.3\end{array}$ & $\begin{array}{l}0.18 \\
0.21 \\
0.13\end{array}$ & $\begin{array}{l}97.52 \\
98.09 \\
98.51\end{array}$ & $<0.01$ & $\begin{array}{l}1.96 \\
1.11 \\
1.10\end{array}$ & $\begin{array}{l}0.29 \\
0.37 \\
0.22\end{array}$ & $\begin{array}{r}832 \\
2090 \\
1350\end{array}$ \\
\hline T3- & $\begin{array}{l}\text { North } \\
\text { Middle } \\
\text { South }\end{array}$ & $\begin{array}{l}11.4 \\
11.5 \\
11.0\end{array}$ & $\begin{array}{l}0.42 \\
0.52 \\
0.34\end{array}$ & $\begin{array}{l}87.17 \\
89.01 \\
92.08\end{array}$ & $<0.01$ & $\begin{array}{r}11.90 \\
9.78 \\
7.09\end{array}$ & $\begin{array}{l}0.49 \\
0.55 \\
0.38\end{array}$ & $\begin{array}{l}3170 \\
5920 \\
3910\end{array}$ \\
\hline T4- & $\begin{array}{l}\text { North } \\
\text { Middle } \\
\text { South }\end{array}$ & $\begin{array}{r}9.8 \\
10.4 \\
9.9\end{array}$ & $\begin{array}{l}0.08 \\
0.49 \\
0.12\end{array}$ & $\begin{array}{l}98.22 \\
97.51 \\
98.38\end{array}$ & $<-\overline{0} 01$ & $\begin{array}{l}1.39 \\
1.21 \\
1.19\end{array}$ & $\begin{array}{l}0.23 \\
0.67 \\
0.22\end{array}$ & $\begin{array}{l}3130 \\
7870 \\
3270\end{array}$ \\
\hline T9- & $\begin{array}{l}\text { North } \\
\text { Middle } \\
\text { South }\end{array}$ & $\begin{array}{l}9.3 \\
9.3 \\
9.1\end{array}$ & $\begin{array}{l}0.41 \\
0.12 \\
0.19\end{array}$ & $\begin{array}{l}97.80 \\
98.79 \\
98.69\end{array}$ & $<-\overline{0.01}$ & $\begin{array}{l}1.08 \\
0.75 \\
0.81 \\
\end{array}$ & $\begin{array}{l}0.52 \\
0.22 \\
0.27\end{array}$ & $\begin{array}{l}2700 \\
2510 \\
2220 \\
\end{array}$ \\
\hline
\end{tabular}


Table 18 Summary of actinide elements in OHF sludge ${ }^{a}$

\begin{tabular}{|c|c|c|c|c|c||}
\hline \multirow{2}{*}{ Actinide } & \multicolumn{1}{|c|}{ T1 } & T2 & T3 & T4 & T9 \\
\cline { 2 - 6 } & $(\% \alpha)$ & $(\% \alpha)$ & $(\% \alpha)$ & $(\% \alpha)$ & $(\% \alpha)$ \\
\hline \hline${ }^{232} \mathrm{Th}$ & 0.08 & 0.07 & 0.11 & 0.08 & 0.07 \\
${ }^{233} \mathrm{U}$ & 1.75 & 1.48 & 5.14 & 4.09 & 1.84 \\
${ }^{234} \mathrm{U}$ & 0.02 & 0.02 & 0.05 & 0.03 & 0.02 \\
${ }^{235} \mathrm{U}$ & $<0.01$ & $<0.01$ & $<0.01$ & $<0.01$ & $<0.01$ \\
${ }^{238} \mathrm{U}$ & 0.01 & $<0.01$ & 0.02 & 0.02 & 0.01 \\
${ }^{237} \mathrm{~Np}$ & $<0.01$ & $<0.01$ & $<0.01$ & $<0.01$ & $<0.01$ \\
${ }^{238} \mathrm{Pu}$ & 6.47 & 4.09 & 3.01 & 3.31 & 13.80 \\
${ }^{239} \mathrm{Pu}$ & 1.22 & 1.06 & 1.11 & 1.08 & 1.59 \\
${ }^{240} \mathrm{Pu}$ & 1.19 & 1.29 & 1.59 & 1.47 & 1.28 \\
${ }^{241} \mathrm{Am}{ }^{\mathrm{b}}$ & 11.55 & 4.92 & 5.04 & 1.34 & 3.74 \\
${ }^{244} \mathrm{Cm}$ & 77.72 & 87.07 & 83.94 & 88.59 & 77.65 \\
\hline \hline Gross $\alpha(\mathrm{Bq} / \mathrm{g})$ & 460000 & 530000 & 300000 & 600000 & 350000 \\
TRU $\alpha(\mathrm{Bq} / \mathrm{g})$ & 92000 & 60000 & 32000 & 43000 & 71000 \\
TRU $\alpha / \alpha(\%)^{\mathrm{c}}$ & 20.0 & 11.3 & 10.7 & 7.2 & 20.3 \\
TRU $\alpha / 3700^{\mathrm{d}}$ & 24.9 & 16.2 & 8.6 & 11.6 & 19.2 \\
\hline
\end{tabular}

This summary is based on the sludge sample taken from the middle of each tank.

${ }^{b}$ The ${ }^{241} \mathrm{Am}$ data is based on subtracting the ${ }^{238} \mathrm{Pu}$ by TIMS from the alpha peak measured at $5.15 \mathrm{MeV}\left({ }^{238} \mathrm{Pu}+{ }^{241} \mathrm{Am}\right)$ in the alpha spectrum.

c This row represents the relative percent of transuranic (TRU) alpha as a function of the total gross alpha.

${ }^{d}$ This row represents the degree of transuranic (TRU) activity as a function of the lower limit for TRU waste of 3700 $\mathrm{Bq} / \mathrm{g}(100 \mathrm{nCi} / \mathrm{g})$.

The distribution of the alpha activity for each OHF sludge sample are summarized in Table 18 as the relative percent alpha for each radionuclide. In general, the alpha activity in the OHF system is strongly weighted by the ${ }^{244} \mathrm{Cm}$ which has the highest specific activity. The list of actinides in Table 18 required several radiochemical and inorganic analytical measurements to generate the best estimates for each of the alpha activities. The ${ }^{232} \mathrm{Th}$ activity was calculated from the total thorium measured by ICP-AES. The other thorium isotopes $\left({ }^{228} \mathrm{Th},{ }^{229} \mathrm{Th}\right.$, and $\left.{ }^{230} \mathrm{Th}\right)$ are present in the ORNL 
sludge waste at such low mass, their presence would not effect the ICP-AES measurements. The uranium isotopes were measured by TIMS. The atom $\%$ results were then converted to weight $\%$ and used to calculate the concentration of each uranium isotope from the total uranium results obtained by ICP-AES. The activity for each uranium radionuclide is then calculated from the specific activity for each isotope. The plutonium isotopes were first measured by TIMS, and then along with the total plutonium alpha activity measured after a chemical separation was used to calculate the activity for each plutonium isotope. The ${ }^{244} \mathrm{Cm}$ was measured directly by alpha spectrometry without any chemical separation. The ${ }^{241} \mathrm{Am}$ activity was determined by subtracting the ${ }^{238} \mathrm{Pu}$ activity from the sum of the ${ }^{238} \mathrm{Pu}+{ }^{241} \mathrm{Am}$ measured by alpha spectrometry. Both ${ }^{238} \mathrm{Pu}$ and ${ }^{241} \mathrm{Am}$ have an alpha energy of about $5.50 \mathrm{MeV}$ and can not be resolved by alpha spectrometry. Due to cost limits there was no chemical separation done to improve the americium data for this project.

Figure 5 Distribution of Major Alpha Emitters in OHF Sludge

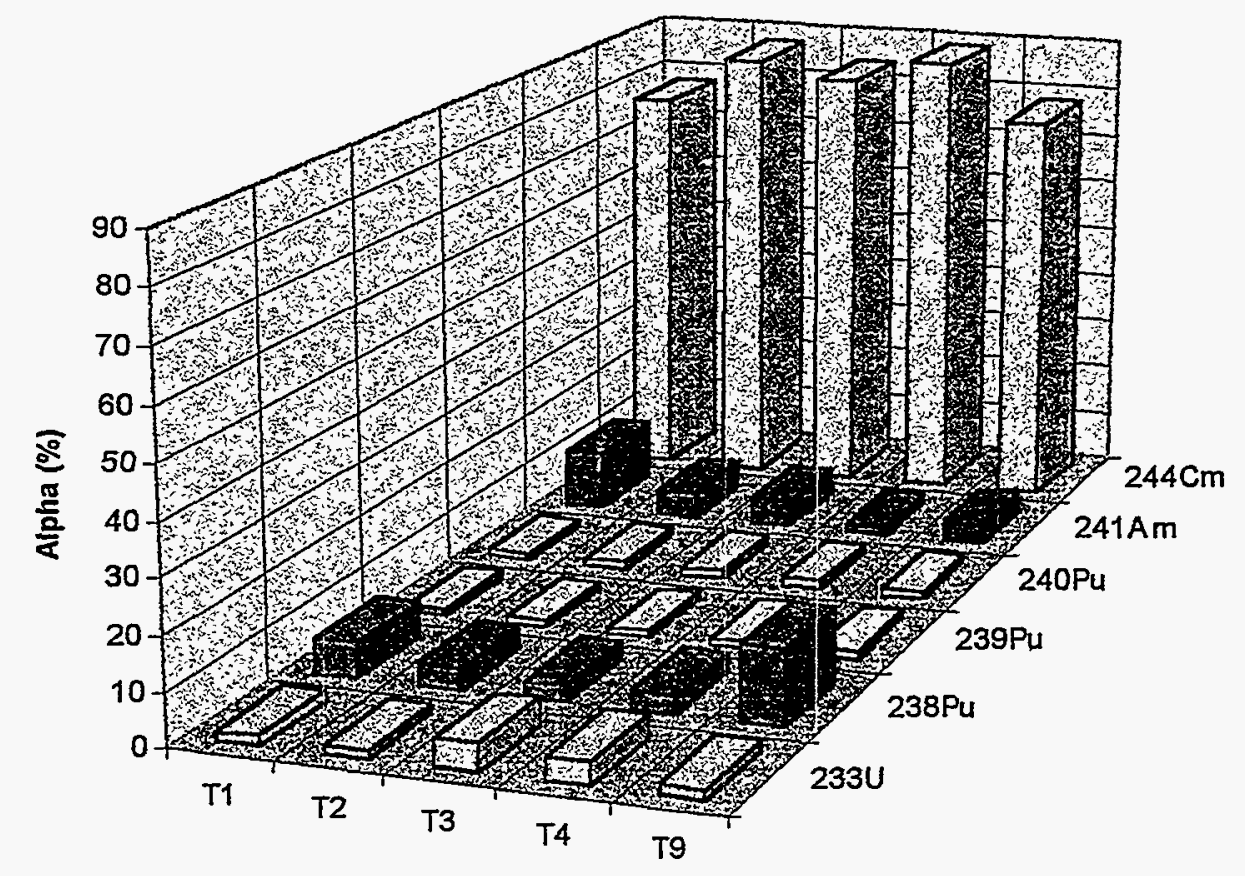




\subsection{Inorganic RCRA Characteristics for the OHF System}

The RCRA regulatory limits for hazardous metals are listed in Table 19. This table includes the limits for both the extract generated by the EPA Toxicity Characteristic Leaching Protocol (TCLP) and the limits for total metals found in a solid or sludge waste. The effective total metal limits for sludge samples are implicitly twenty times higher than the TCLP extraction limits due to the 1:20 dilution used for the TCLP extraction procedure. In other words, the RCRA metals must be present at twenty times the regulatory limit for the TCLP extract limits to be exceeded, even if all the solid sample dissolves.

\section{Table 19 Summary of RCRA regulatory limits}

\begin{tabular}{|ll|c|c||}
\hline Metals & & $\begin{array}{c}\text { TCLP Extract } \\
\text { and Liquids } \\
(\mathbf{m g} / \mathbf{L})\end{array}$ & $\begin{array}{c}\text { Solid/Sludge } \\
\text { Total Metal } \\
(\mathbf{m g} / \mathbf{K g})\end{array}$ \\
\hline Silver & $(\mathrm{Ag})$ & 5 & 100 \\
Arsenic & $(\mathrm{As})$ & 5 & 100 \\
Barium & $(\mathrm{Ba})$ & 100 & 2000 \\
Cadmium & $(\mathrm{Cd})$ & 1 & 20 \\
Chromium & $(\mathrm{Cr})$ & 5 & 100 \\
Mercury & $(\mathrm{Hg})$ & 0.2 & 4 \\
Nickel & $(\mathrm{Ni})$ & 50 & 1000 \\
Lead & $(\mathrm{Pb})$ & 5 & 100 \\
Selenium & $(\mathrm{Se})$ & 1 & 20 \\
Thallium & $(\mathrm{Tl})$ & 0.9 & 18 \\
\hline
\end{tabular}

If the RCRA metal concentrations are found to be below the total metal limits, the solid waste can not fail the TCLP leach test. If the RCRA metal concentrations exceed the total metal limits, the TCLP leach test must be done to determine if the solid waste is hazardous. For solid samples, the TCLP leach test is only valid for the final waste form ready for disposal. The total metal concentration data can be used as acceptable process knowledge if the final waste form only results in a dilution of the RCRA metal concentrations. Examples of waste forms that result in a dilution 
of a solid waste includes grouting ( 2 fold dilution) and vitrification ( 3 fold dilution). If the total metal limit is exceeded after stabilizing the waste, the TCLP leach test would be required for only the metals that had the potential to exceed the regulatory limits.

All of the OHF supernatant samples exceed the RCRA regulatory limits for mercury and two of the tanks (T3 and T4) exceeded the limit for chromium. The current technology used for long term storage of the liquid waste is a solidification process that usually results in a final waste form that passes the TCLP leach test. In general, the OHF sludge samples exceed the total metal RCRA limits for chromium, lead, and mercury. Most of the ORNL radioactive waste sludge samples characterized to date have exceeded the total metal limits for these RCRA metals. Based on past experience, it is expected that solidification of the ORNL sludge would fix these RCRA metals such that the final waste form would pass a TCLP leach test. The nickel and thallium are proposed RCRA metals and are included in the data for future waste management decisions.

\subsection{TRU Classifications for OHF Waste System}

The DOE definition for Transuranic (TRU) Waste includes the following conditions,

- TRU activity $\geq 3700 \mathrm{~Bq} / \mathrm{g}(100 \mathrm{nCi} / \mathrm{g})$,

- TRU isotopes must be alpha emitting actinides with $Z>92$ (uranium),

- TRU isotopes must have a half life $z 20$ years.

This definition excludes all thorium and uranium isotopes. The short lived actinide ${ }^{244} \mathrm{Cm}\left(\mathrm{t}_{1 / 2}=18.1\right.$ years), which is common to ORNL waste, falls outside the TRU definition. Also, the plutonium isotope, ${ }^{241} \mathrm{Pu}$, would be excluded from calculation of the TRU activity because it is a pure beta emitter. The primary actinide elements present in ORNL waste at sufficient levels to meet the TRU definition include ${ }^{238} \mathrm{Pu},{ }^{239} \mathrm{Pu},{ }^{240} \mathrm{Pu}$, and ${ }^{241} \mathrm{Am}$. There is some current work at the Radiochemical Engineering Development Center (Mark-42 fuel assembly processing) that could generate enough ${ }^{243} \mathrm{Am}$ to make a significant contribution to TRU alpha content of the waste, but this contribution to the alpha content will not impact the OHF tanks. The remaining actinide elements present in ORNL waste are generally not available at high enough activity, and/or do not have a long enough half-life to meet the TRU definition. 
None of the OHF supernatant samples discussed in this report had enough alpha activity to be considered as TRU waste. All of the OHF sludge samples characterized for this project were classified as TRU waste based on only the plutonium and americium activity. The alpha activity reported is based on wet weight and if adjusted for dry weight the TRU activity would increase a factor of two or more. The OHF sludge samples contained enough plutonium and americium activity to easily satisfy the WIPP waste acceptance criteria ${ }^{12}$ for transuranic waste. Based on the TRU activity (see last row in Table 18), any dilution of the sludge that would result from a solidification process such as grouting or vitrification would most likely not effect the TRU classification.

\subsection{Distribution of Fissile Material in OHF Waste System}

The ORNL LLLW waste acceptance criteria (WAC) requires the fissile isotopes of uranium and plutonium to be diluted with ${ }^{238} \mathrm{U}$ and ${ }^{232} \mathrm{Th}$, respectively. A summary of the major isotopes of uranium found in the supernatant are provided in Table 20. The dilution or "denature" ratios for these OHF supernatant samples are summarized in Table 21. None of the dilution factors determined for the OHF supernatant samples satisfied the current administrative control limits for criticality.

A summary of the major isotopes of uranium found in the OHF sludge are provided in Table 22 along with Table 23 which summarizes the dilution or "denature" ratios for these samples. Similar to the OHF supernatant, none of the dilution factors determined for the OHF sludge samples satisfied the current administrative control limits for criticality

All the dilution ratios listed in Table 20-23 are based on equations discussed in section 3.5 of this report. Also, these tables include uranium isotopic data measured by ICP-MS for comparison to the more accurate data by TIMS. The ICP-MS data is provided to support future use of the technique for quick-turnaround and lower cost measurements of uranium isotopes. Although TIMS is the preferred technique for these measurements, many projects that do not require the high level of accuracy and precision provided by TIMS, may choose to take advantage of the lower cost and fast results provided by ICP-MS. 
Table 20 Summary of uranium isotopics for OHF supernatant

\begin{tabular}{|c|c|c|c|c|c|c|c|c|c|}
\hline \multirow{2}{*}{\multicolumn{2}{|c|}{ Tank }} & \multicolumn{2}{|c|}{$\begin{array}{c}{ }^{233} \mathrm{U} \\
\text { (atom \%) }\end{array}$} & \multicolumn{2}{|c|}{$\begin{array}{c}{ }^{235} \mathrm{U} \\
\text { (atom \%) }\end{array}$} & \multicolumn{2}{|c|}{$\begin{array}{c}{ }^{238} U \\
(\text { atom \%) }\end{array}$} & \multirow{2}{*}{$\begin{array}{c}\mathrm{U} \\
(\mathrm{mg} / \mathrm{L})\end{array}$} & \multirow[t]{2}{*}{ pH } \\
\hline & & TIMS & ICP-MS & TIMS & ICP-MS & TIMS & ICP-MS & & \\
\hline T1- & $\begin{array}{l}\text { North } \\
\text { Middle } \\
\text { South }\end{array}$ & $\begin{array}{l}0.345 \\
0.350 \\
0.338\end{array}$ & $\begin{array}{c}0.343 \\
- \\
0.339\end{array}$ & $\begin{array}{l}0.578 \\
0.560 \\
0.568\end{array}$ & $\begin{array}{c}0.573 \\
- \\
0.564\end{array}$ & $\begin{array}{l}99.059 \\
99.070 \\
99.077\end{array}$ & $\begin{array}{c}99.069 \\
- \\
99.079\end{array}$ & $\begin{array}{l}246 \\
281 \\
254\end{array}$ & $\begin{array}{l}8.9 \\
9.3 \\
8.9\end{array}$ \\
\hline T2- & $\begin{array}{l}\text { North } \\
\text { Middle } \\
\text { South }\end{array}$ & $\begin{array}{l}0.354 \\
0.350 \\
0.354\end{array}$ & $\begin{array}{c}0.352 \\
- \\
0.353\end{array}$ & $\begin{array}{l}0.497 \\
0.480 \\
0.498\end{array}$ & $\begin{array}{c}0.496 \\
- \\
0.494\end{array}$ & $\begin{array}{l}99.131 \\
99.150 \\
99.130\end{array}$ & $\begin{array}{c}99.134 \\
- \\
99.135\end{array}$ & $\begin{array}{l}221 \\
219 \\
206\end{array}$ & $\begin{array}{l}9.3 \\
9.3 \\
9.3\end{array}$ \\
\hline T3- & $\begin{array}{l}\text { North } \\
\text { Middle } \\
\text { South }\end{array}$ & $\begin{array}{l}0.188 \\
0.220 \\
0.182\end{array}$ & $\begin{array}{c}0.167 \\
- \\
0.192\end{array}$ & $\begin{array}{l}0.614 \\
0.570 \\
0.616\end{array}$ & $\begin{array}{c}0.605 \\
- \\
0.620\end{array}$ & $\begin{array}{l}99.182 \\
99.190 \\
99.186\end{array}$ & $\begin{array}{c}99.216 \\
- \\
99.175\end{array}$ & $\begin{array}{l}7.72 \\
0.39 \\
7.74\end{array}$ & $\begin{array}{r}9.5 \\
11.6 \\
9.6\end{array}$ \\
\hline T4- & $\begin{array}{l}\text { North } \\
\text { Middle } \\
\text { South }\end{array}$ & $\begin{array}{l}0.368 \\
0.350 \\
0.369\end{array}$ & $\begin{array}{c}0.367 \\
- \\
0.366\end{array}$ & $\begin{array}{l}0.573 \\
0.530 \\
0.581\end{array}$ & $\begin{array}{c}0.575 \\
- \\
0.574\end{array}$ & $\begin{array}{l}99.040 \\
99.100 \\
99.031\end{array}$ & $\begin{array}{c}99.040 \\
- \\
99.041\end{array}$ & $\begin{array}{l}216 \\
195 \\
215\end{array}$ & $\begin{array}{l}10.1 \\
10.4 \\
10.1\end{array}$ \\
\hline T9- & $\begin{array}{l}\text { North } \\
\text { Middle } \\
\text { South }\end{array}$ & $\begin{array}{l}0.446 \\
0.440 \\
0.447\end{array}$ & $\begin{array}{c}0.444 \\
- \\
0.440\end{array}$ & $\begin{array}{l}0.546 \\
0.520 \\
0.551\end{array}$ & $\begin{array}{c}0.538 \\
- \\
0.537\end{array}$ & $\begin{array}{l}98.988 \\
99.020 \\
98.983\end{array}$ & $\begin{array}{c}98.998 \\
- \\
99.003\end{array}$ & $\begin{array}{l}269 \\
303 \\
275\end{array}$ & $\begin{array}{l}8.9 \\
9.1 \\
8.9\end{array}$ \\
\hline
\end{tabular}

Table 21 Summary of denature ratios ${ }^{a}$ for OHF supernatant

\begin{tabular}{|c|c|c|c|c|c|c|}
\hline & Ink & $\begin{array}{c}{ }^{238} U{ }^{235} U f_{35} \\
\text { Limit }>110 \\
(\text { eq. } 1)\end{array}$ & $\begin{array}{c}{ }^{238} U{ }^{235} U \\
\text { Limit }>110 \\
(\text { eq. } 3)\end{array}$ & $\begin{array}{c}{ }^{238} U{ }^{233} U \\
\text { Limit }>200 \\
(\text { eq. 4) }\end{array}$ & $\begin{array}{c}{ }^{232} \mathrm{Th} /{ }^{239} \mathrm{Pu} \\
\text { Limit }>200 \\
(\text { eq. 2) }\end{array}$ & $\mathbf{p H}$ \\
\hline T1- & $\begin{array}{l}\text { North } \\
\text { Middle } \\
\text { South }\end{array}$ & $\begin{array}{l}96 \\
98 \\
98\end{array}$ & $\begin{array}{l}55 \\
55 \\
59\end{array}$ & $\begin{array}{l}107 \\
112 \\
113\end{array}$ & 237 & $\begin{array}{l}8.9 \\
9.3 \\
8.9\end{array}$ \\
\hline T2- & $\begin{array}{l}\text { North } \\
\text { Middle } \\
\text { South }\end{array}$ & $\begin{array}{l}103 \\
106 \\
103\end{array}$ & $\begin{array}{l}61 \\
65 \\
61\end{array}$ & $\begin{array}{l}130 \\
137 \\
130\end{array}$ & 785 & $\begin{array}{l}9.3 \\
9.3 \\
9.3\end{array}$ \\
\hline T3- & $\begin{array}{l}\text { North } \\
\text { Middle } \\
\text { South }\end{array}$ & $\begin{array}{l}116 \\
116 \\
117\end{array}$ & $\begin{array}{l}103 \\
100 \\
104\end{array}$ & $\begin{array}{l}177 \\
173 \\
181\end{array}$ & - & $\begin{array}{c}9.5 \\
11.6 \\
9.6\end{array}$ \\
\hline T4- & $\begin{array}{l}\text { North } \\
\text { Middle } \\
\text { South }\end{array}$ & $\begin{array}{c}94 \\
101 \\
93\end{array}$ & $\begin{array}{l}48 \\
58 \\
47\end{array}$ & $\begin{array}{c}102 \\
121 \\
99\end{array}$ & - & $\begin{array}{l}10.1 \\
10.4 \\
10.1\end{array}$ \\
\hline T9- & $\begin{array}{l}\text { North } \\
\text { Middle } \\
\text { South }\end{array}$ & $\begin{array}{l}88 \\
90 \\
87\end{array}$ & $\begin{array}{l}22 \\
25 \\
21\end{array}$ & $\begin{array}{l}91 \\
99 \\
89\end{array}$ & $\begin{array}{l}- \\
-\end{array}$ & $\begin{array}{l}8.9 \\
9.1 \\
8.9\end{array}$ \\
\hline
\end{tabular}

${ }^{2}$ All ratios calculated with TIMS data.

${ }^{b}$ Concentration of thorium to low to calculate ratio. 
Table 22 Summary of uranium isotopics for OHF sludge

\begin{tabular}{|c|c|c|c|c|c|c|c|c|c|}
\hline \multirow{2}{*}{\multicolumn{2}{|c|}{ Tank }} & \multicolumn{2}{|c|}{$\begin{array}{c}{ }^{233} \mathrm{U} \\
\text { (atom \%) }\end{array}$} & \multicolumn{2}{|c|}{$\begin{array}{c}{ }^{235} \mathrm{U} \\
\text { (atom \%) }\end{array}$} & \multicolumn{2}{|c|}{$\begin{array}{c}{ }^{238} U \\
\text { (atom \%) }\end{array}$} & \multirow{2}{*}{$\begin{array}{c}\mathrm{U} \\
(\mathrm{mg} / \mathrm{L})\end{array}$} & \multirow[t]{2}{*}{$\mathbf{p H}$} \\
\hline & & TIMS & ICP-MS & TIMS & ICP-MS & TIMS & ICP-MS & & \\
\hline T1- & $\begin{array}{l}\text { North } \\
\text { Middle } \\
\text { South }\end{array}$ & $\begin{array}{l}1.234 \\
0.930 \\
0.634\end{array}$ & $\begin{array}{l}1.216 \\
- \\
0.632\end{array}$ & $\begin{array}{l}0.378 \\
0.430 \\
0.506\end{array}$ & $\begin{array}{l}0.378 \\
- \\
0.504\end{array}$ & $\begin{array}{l}98.363 \\
98.610 \\
98.839\end{array}$ & $\begin{array}{c}98.378 \\
- \\
98.843\end{array}$ & $\begin{array}{l}1410 \\
2420 \\
1360\end{array}$ & $\begin{array}{l}8.9 \\
9.6 \\
9.0\end{array}$ \\
\hline T2- & $\begin{array}{l}\text { North } \\
\text { Middle } \\
\text { South }\end{array}$ & $\begin{array}{l}0.961 \\
1.070 \\
0.912\end{array}$ & $\begin{array}{l}0.950 \\
- \\
0.915\end{array}$ & $\begin{array}{l}0.393 \\
0.350 \\
0.402\end{array}$ & $\begin{array}{l}0.399 \\
- \\
0.396\end{array}$ & $\begin{array}{l}98.623 \\
98.550 \\
98.662\end{array}$ & $\begin{array}{c}98.623 \\
- \\
98.664\end{array}$ & $\begin{array}{r}832 \\
2090 \\
1350\end{array}$ & $\begin{array}{l}9.2 \\
9.6 \\
9.3\end{array}$ \\
\hline T3- & $\begin{array}{l}\text { North } \\
\text { Middle } \\
\text { South }\end{array}$ & $\begin{array}{l}0.693 \\
0.740 \\
0.723\end{array}$ & $\begin{array}{l}0.688 \\
- \\
0.738\end{array}$ & $\begin{array}{l}0.430 \\
0.400 \\
0.440\end{array}$ & $\begin{array}{l}0.433 \\
- \\
0.442\end{array}$ & $\begin{array}{l}98.857 \\
98.840 \\
98.817\end{array}$ & $\begin{array}{c}98.859 \\
- \\
98.800\end{array}$ & $\begin{array}{l}3170 \\
5920 \\
3910\end{array}$ & $\begin{array}{l}11.4 \\
11.5 \\
11.0\end{array}$ \\
\hline T4- & $\begin{array}{l}\text { North } \\
\text { Middle } \\
\text { South }\end{array}$ & $\begin{array}{l}0.498 \\
0.890 \\
0.605\end{array}$ & $\begin{array}{l}0.501 \\
- \\
0.613\end{array}$ & $\begin{array}{l}0.479 \\
0.380 \\
0.516\end{array}$ & $\begin{array}{l}0.478 \\
- \\
0.517\end{array}$ & $\begin{array}{l}99.006 \\
98.710 \\
98.858\end{array}$ & $\begin{array}{c}99.002 \\
- \\
98.851\end{array}$ & $\begin{array}{l}3130 \\
7870 \\
3270\end{array}$ & $\begin{array}{r}9.8 \\
10.4 \\
9.9\end{array}$ \\
\hline T9- & $\begin{array}{l}\text { North } \\
\text { Middle } \\
\text { South }\end{array}$ & $\begin{array}{l}1.198 \\
0.730 \\
0.944\end{array}$ & $\begin{array}{l}1.192 \\
- \\
0.941\end{array}$ & $\begin{array}{l}0.377 \\
0.500 \\
0.449\end{array}$ & $\begin{array}{l}0.374 \\
- \\
0.445\end{array}$ & $\begin{array}{l}98.399 . \\
98.750 \\
98.583\end{array}$ & $\begin{array}{c}98.410 \\
- \\
98.590\end{array}$ & $\begin{array}{l}2700 \\
2510 \\
2220\end{array}$ & $\begin{array}{l}9.3 \\
9.3 \\
9.1\end{array}$ \\
\hline
\end{tabular}

Table 23 . Summary of denature ratios ${ }^{a}$ for OHF sludge

\begin{tabular}{|c|c|c|c|c|c|c|}
\hline \multicolumn{2}{|c|}{ Tank } & $\begin{array}{c}{ }^{238} \mathrm{U} /{ }^{235} \mathrm{U} f_{35} \\
\text { Limit }>110 \\
(\text { eq. } 1)\end{array}$ & $\begin{array}{c}{ }^{238} U{ }^{235} U \\
\text { Limit > } 110 \\
\text { (eq. 3) }\end{array}$ & $\begin{array}{c}{ }^{238} U^{233} U \\
\text { Limit }>200 \\
(\text { eq. 4) }\end{array}$ & $\begin{array}{c}{ }^{232} \mathrm{Th}{ }^{239} \mathbf{P u} \\
\text { Limit }>200 \\
(\mathrm{eq} \cdot 2)^{\mathrm{b}}\end{array}$ & pH \\
\hline T1- & $\begin{array}{l}\text { North } \\
\text { Middle } \\
\text { South }\end{array}$ & $\begin{array}{l}49 \\
60 \\
74\end{array}$ & $\begin{array}{l}-384 \\
-197 \\
-51\end{array}$ & $\begin{array}{l}47 \\
57 \\
71\end{array}$ & $\begin{array}{l}20100 \\
37800 \\
15800\end{array}$ & $\begin{array}{l}8.9 \\
9.6 \\
9.0\end{array}$ \\
\hline T2- & $\begin{array}{l}\text { North } \\
\text { Middle } \\
\text { South }\end{array}$ & $\begin{array}{l}59 \\
56 \\
62\end{array}$ & $\begin{array}{l}-231 \\
-321 \\
-201\end{array}$ & $\begin{array}{l}59 \\
58 \\
62\end{array}$ & $\begin{array}{l}14600 \\
38800 \\
17500\end{array}$ & $\begin{array}{l}9.2 \\
9.6 \\
9.3\end{array}$ \\
\hline T3- & $\begin{array}{l}\text { North } \\
\text { Middle } \\
\text { South }\end{array}$ & $\begin{array}{l}74 \\
72 \\
71 \\
\end{array}$ & $\begin{array}{c}-87 \\
-117 \\
-98 \\
\end{array}$ & $\begin{array}{l}77 \\
76 \\
72 \\
\end{array}$ & $\begin{array}{l}23000 \\
53800 \\
24400\end{array}$ & $\begin{array}{l}11.4 \\
11.5 \\
11.0 \\
\end{array}$ \\
\hline T4- & $\begin{array}{l}\text { North } \\
\text { Middle } \\
\text { South }\end{array}$ & $\begin{array}{l}88 \\
66 \\
76\end{array}$ & $\begin{array}{c}3 \\
-201 \\
-38\end{array}$ & $\begin{array}{l}96 \\
66 \\
72\end{array}$ & $\begin{array}{l}17300 \\
44200 \\
16500\end{array}$ & $\begin{array}{c}9.8 \\
10.4 \\
9.9\end{array}$ \\
\hline T9- & $\begin{array}{l}\text { North } \\
\text { Middle } \\
\text { South }\end{array}$ & $\begin{array}{l}50 \\
68 \\
58\end{array}$ & $\begin{array}{l}-366 \\
-89 \\
-195\end{array}$ & $\begin{array}{l}49 \\
62 \\
54\end{array}$ & $\begin{array}{l}23800 \\
23600 \\
17000\end{array}$ & $\begin{array}{l}9.3 \\
9.3 \\
9.1\end{array}$ \\
\hline
\end{tabular}

All ratios calculated with TIMS data.

' The thorium to plutonium ratio for the middle sludge sample is based on the ${ }^{239} \mathrm{Pu}$ weight $\%$ determined by mass spectrometry, the ratios for the north and south samples are conservative values based the ${ }^{239} \mathrm{Pu}+{ }^{240} \mathrm{Pu}$ determined by alpha spectrometry. 
The dilution ratios listed in Tables 21 and 23 are based on the ratio of weight $\%$, not the ratio of atom $\%$ given in the data tables. There is a small difference between atom $\%$, reported for the uranium and plutonium, and weight $\%$, which is needed for many calculations performed with the analytical data. To convert from atom $\%$ to weight $\%$, we used the following equation,

$$
\begin{aligned}
& W_{i}=\frac{a_{i} M_{i}}{\sum_{i}^{n} a_{i} M_{i}} X 100 \% \\
& \text { where, } \quad \mathrm{W}_{\mathrm{i}} \quad=\text { weight } \% \text {, } \\
& \mathrm{M}_{\mathrm{i}} \quad=\text { nuclidic mass } \\
& \mathrm{a}_{\mathrm{i}} \quad=\text { atom } \% \text {. }
\end{aligned}
$$

An example of this calculation is provided in Table 24, which shows there is not much difference between the atom $\%$ and the weight $\%$.

Table 24 Example of converting atom \% to weight \% for typical sludge

\begin{tabular}{|c|c|r|r|r|}
\hline Isotope & $\begin{array}{c}\text { Nuclidic mass } \\
\text { (g/mol) }\end{array}$ & atom \% & $\left.\mathbf{( a}_{\mathbf{i}} \mathbf{M}_{\mathbf{i}}\right)$ & weight \% \\
\hline \hline${ }^{233} \mathrm{U}$ & 233.039629 & 0.056 & 13.0502 & 0.0548 \\
${ }^{234} \mathrm{U}$ & 234.040947 & 0.004 & 0.9362 & 0.0039 \\
${ }^{233} \mathrm{U}$ & 235.043924 & 0.621 & 145.9623 & 0.6132 \\
${ }^{236} \mathrm{U}$ & 236.045563 & 0.002 & 0.4721 & 0.0020 \\
${ }^{238} \mathrm{U}$ & 238.050785 & 99.316 & 23642.2518 & 99.3260 \\
& & & & \\
& & 99.999 & 23802.6726 & 99.9999 \\
\hline
\end{tabular}

The typical distribution of plutonium isotopes by alpha activity are illustrated in Fig. 6 for each of the OHF samples. For comparison, Fig. 7 shows the distribution of the plutonium isotopes by concentration. The data presented in Fig. 6 and Fig. 7 represents the OHF sludge samples taken from the center access port in each tank. One should note that the ${ }^{238} \mathrm{Pu}$ dominates the alpha activity and the ${ }^{239} \mathrm{Pu}$ is the major isotope by weight or concentration. 
Figure 6 Distribution of Plutonium by Alpha Activity in the OHF Sludge

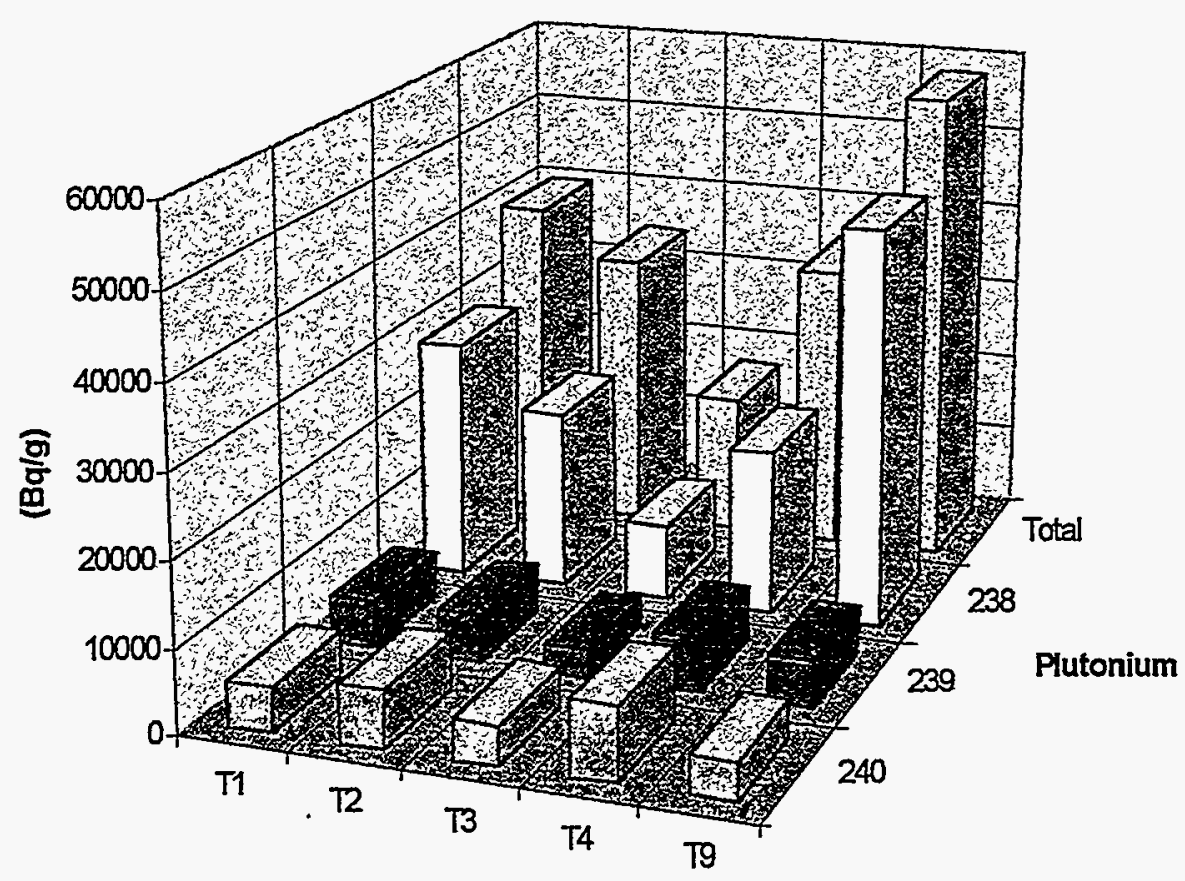

Figure 7 Distribution of Plutonium by Concentration in the OHF Sludge

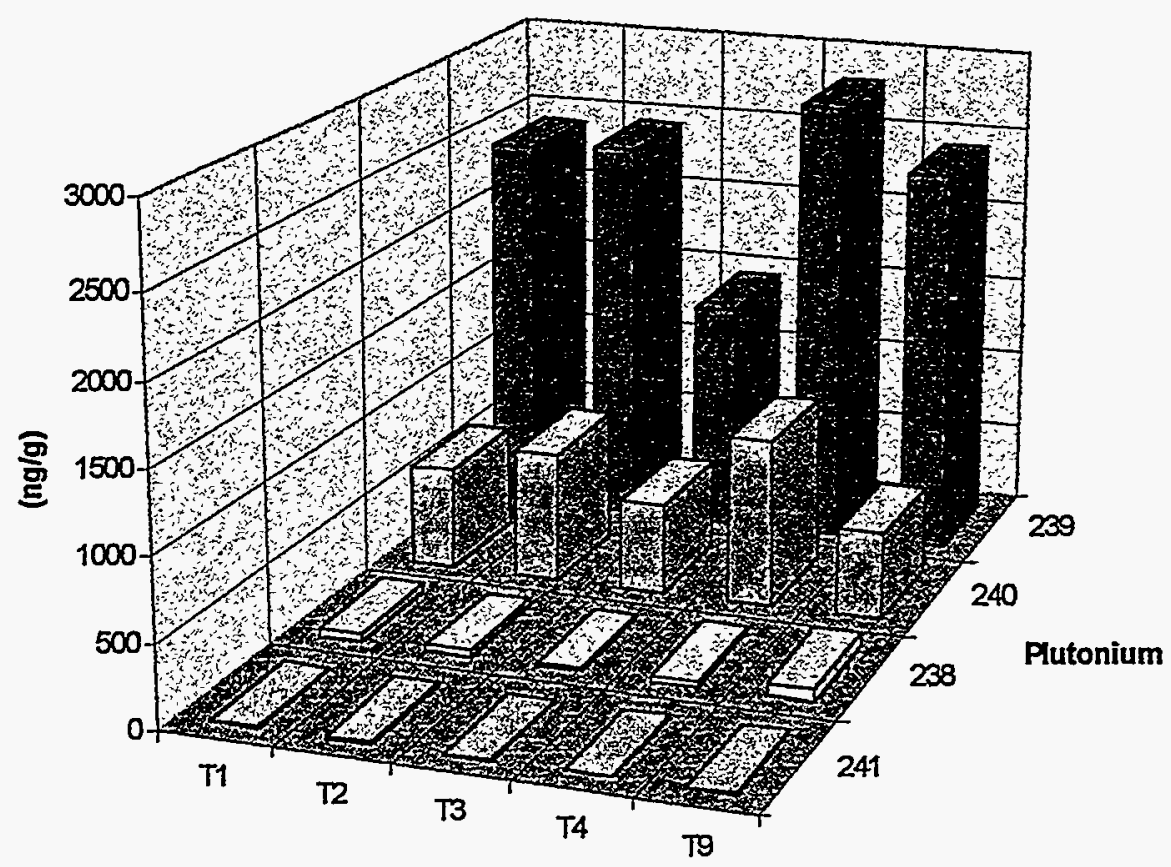


Even though the "denature" ratios for ${ }^{233} \mathrm{U}$ and ${ }^{235} \mathrm{U}$ are outside the administrative control limits, the high level of thorium present in the OHF tanks provides a broad safety margin and a high degree of confidence that the criticality concerns are satisfied as long as the tanks are maintained within the typical chemical environment conditions (aqueous system with $\mathrm{pH}>11$ ) used routinely for the ORNL liquid waste system. The ${ }^{232} \mathrm{Th}$ is actually a better neutron poison than ${ }^{238} \mathrm{U}$, considering the thermal neutron capture cross sections of $\sigma_{\gamma}=7.37 \mathrm{~b}$ and $\sigma_{\gamma}=2.68 \mathrm{~b}$, respectively. If the average chemical environment for the waste is modified the thorium may no longer be a good "denature" agent for the fissile uranium present, because at lower $\mathrm{pH}$ levels the thorium may not chemically behave similar to uranium.

Although the contents of the OHF tanks could be safely transferred to the MVST system and the resulting average isotopic distribution for uranium in the MVST after the transfer would meet the administrative control limits, the waste transfer would still violate the current waste acceptance criteria for the MVST system. Any transfer of the OHF inventory without prior treatment to increase the "denature" ratios would require a variance supported by a detailed criticality safety review. The cost of the safety review could easily exceed the cost of adding depleted uranium before any transfers to the MVST system. Therefore, it is recommended that the "denature" ratios be adjusted with depleted uranium before any transfers to the MVST system. 


\subsection{Discussion of the Total Anion Content in the Sludge}

The anion data for the OHF sludge samples collected from three locations in each tank are summarized in Table 25 in a format that allows easy comparison of the results. This anion data is based on a water wash of the sludge followed by analysis of the clarified water by ion chromatography. This anion data should not be considered to be representative of the total anion content for each sludge sample.

Table 25 Summary of anionic species observed in the OHF sludge

\begin{tabular}{|c|c|c|c|c|c|c|}
\hline \multirow[t]{2}{*}{ Anion } & & \multicolumn{5}{|c|}{ (mg/Kg) } \\
\hline & & $\mathrm{T} 1$ & $\mathbf{T} 2$ & T3 & $\mathrm{T} 4$ & T9 \\
\hline Acetate & $\begin{array}{l}\text { North } \\
\text { Middle } \\
\text { South }\end{array}$ & $\begin{array}{r}<5 \\
<5\end{array}$ & $\begin{array}{r}<5 \\
<5\end{array}$ & $\begin{array}{c}56.2 \\
- \\
57.1\end{array}$ & $\begin{array}{c}12.9 \\
- \\
18.8\end{array}$ & $\begin{array}{r}<5 \\
<-\end{array}$ \\
\hline Bromide & $\begin{array}{l}\text { North } \\
\text { Middle } \\
\text { South }\end{array}$ & $\begin{array}{l}<4.8 \\
<4.6 \\
<4.8\end{array}$ & $\begin{array}{l}<4.8 \\
<5.2 \\
<4.8\end{array}$ & $\begin{array}{c}40.1 \\
<43 \\
19.7\end{array}$ & $\begin{array}{l}<4.8 \\
<41 \\
<4.6\end{array}$ & $\begin{array}{l}32.7 \\
70.0 \\
26.1\end{array}$ \\
\hline Chloride & $\begin{array}{l}\text { North } \\
\text { Middle } \\
\text { South }\end{array}$ & $\begin{array}{l}283 \\
247 \\
296\end{array}$ & $\begin{array}{l}536 \\
366 \\
494\end{array}$ & $\begin{array}{r}1160 \\
947 \\
1130\end{array}$ & $\begin{array}{l}461 \\
401 \\
462\end{array}$ & $\begin{array}{l}3700 \\
3760 \\
3240\end{array}$ \\
\hline Fluoride & $\begin{array}{l}\text { North } \\
\text { Middle } \\
\text { South }\end{array}$ & $\begin{array}{l}181 \\
176 \\
117\end{array}$ & $\begin{array}{l}138 \\
233 \\
161\end{array}$ & $\begin{array}{l}198 \\
257 \\
207\end{array}$ & $\begin{array}{l}112 \\
272 \\
133\end{array}$ & $\begin{array}{c}82.1 \\
140 \\
87.8\end{array}$ \\
\hline Nitrate & $\begin{array}{l}\text { North } \\
\text { Middle } \\
\text { South }\end{array}$ & $\begin{array}{l}29.6 \\
52.6 \\
46.7\end{array}$ & $\begin{array}{c}<10 \\
27.9 \\
<10\end{array}$ & $\begin{array}{l}5000 \\
4250 \\
4323\end{array}$ & $\begin{array}{l}2020 \\
1470 \\
1660\end{array}$ & $\begin{array}{l}845 \\
869 \\
748\end{array}$ \\
\hline Nitrite & $\begin{array}{l}\text { North } \\
\text { Middle } \\
\text { South }\end{array}$ & $\begin{array}{r}1030 \\
629 \\
1080\end{array}$ & $\begin{array}{r}1200 \\
576 \\
944\end{array}$ & $\begin{array}{l}8240 \\
4670 \\
8528\end{array}$ & $\begin{array}{l}2610 \\
1460 \\
2920\end{array}$ & $\begin{array}{l}324 \\
219 \\
272\end{array}$ \\
\hline Oxalate & $\begin{array}{l}\text { North } \\
\text { Middle } \\
\text { South }\end{array}$ & $\begin{array}{r}<5 \\
- \\
303\end{array}$ & $\begin{array}{c}<5 \\
- \\
23.9\end{array}$ & $\begin{array}{r}1160 \\
1150\end{array}$ & $\begin{array}{r}913 \\
649\end{array}$ & $\begin{array}{r}<5 \\
282\end{array}$ \\
\hline Phosphate & $\begin{array}{l}\text { North } \\
\text { Middle } \\
\text { South }\end{array}$ & $\begin{array}{l}<10 \\
<10 \\
<10\end{array}$ & $\begin{array}{l}<10 \\
<21 \\
<10\end{array}$ & $\begin{array}{r}<10 \\
<170 \\
<10\end{array}$ & $\begin{array}{r}<10 \\
<170 \\
<10\end{array}$ & $\begin{array}{r}<10 \\
<200 \\
<9\end{array}$ \\
\hline Sulfate & $\begin{array}{l}\text { North } \\
\text { Middle } \\
\text { South }\end{array}$ & $\begin{array}{l}348 \\
339 \\
365^{\circ}\end{array}$ & $\begin{array}{r}1000 \\
726 \\
908\end{array}$ & $\begin{array}{l}3280 \\
2960 \\
3430\end{array}$ & $\begin{array}{l}1190 \\
1210 \\
1360\end{array}$ & $\begin{array}{l}558 \\
616 \\
503\end{array}$ \\
\hline
\end{tabular}


The nitrate and nitrite compounds present in the OHF waste sludge readily dissolve in water and should be well accounted for in the water leaches. This can be argued by looking at the mass balance for the sludge analysis which show acceptable agreement between the total solids measured directly and the sum of the expected compounds in the sludge. The higher levels of nitrite relative to the nitrate present in these OHF samples has not been observed in other ORNL waste tanks and represents a different chemical environment for this waste.

It is believed that a large fraction of the phosphate in the OHF sludge may be present as tributyl phosphate and degradation products dibutyl-and monobutyl phosphate. The tributyl phosphate has low solubility in water and would not be seen in the water leach, which is consistent with the data shown in Table 25 where phosphate values are below the detection limit of the instrument. The phosphate can be estimated based on the results from the total phosphorus by the ICP-AES measurements after a closed vessel microwave acid digestion of the sludge. However, the major source of phosphorous in the OHF tanks does not appear to be phosphate or tributyl phosphate. The ICP-AES values are currently considered to be the acceptable for the total phosphorus in the sludge but give no good indication of total phosphate or tributyl phosphate.

There is no ideal method to measure the total anion content on most sludge samples. The water leaches are considered to be adequate for nitrate, nitrite, and the halides. The total phosphate and sulfate content however may not be obtained with a water leach and any method used that oxidizes the sample would represent a total phosphorus or total sulfur. The best preparation method for total phosphorus or sulfur appears to be closed vessel microwave digestion followed by analysis by ICPAES or ICP-MS. However, in the past total digestion techniques have been subject to contamination problems with many of these common inorganic anions. The subject of the total anion content in sludge samples continues to be in the developmental stages and additional attention is needed if this type of data is required for future waste stabilization processes such as grouting and vitrification. 


\subsection{Solubility of OHF Sludge in Water}

The OHF sludge samples were taken through a water wash to determine the water soluble anions and measure the effect of the sludge on $\mathrm{pH}$. Since this water leach solutions were available, several of the lower cost analytical measurements, including the metals by ICP-AES, gross alpha/beta, and gamma emitters, were measured on the wash solutions from the sludge samples collected from the center of each tank. These additional measurements were performed to evaluate the relative solubility of the sludge in water and to provide additional data for comparison to the advanced washing experiments being performed on Hanford and ORNL sludge by CTD staff. The washing experiment support new waste processing options designed to reduce the volume of TRU waste generated.

The water wash experiment consisted of taking 5 grams of wet sludge and diluting the sample to 50 $\mathrm{mL}$ with deionized water. The sludge was leached with the water on a vortex mixer for several minutes and the clarified liquid was then removed for analysis. Results from the water leaching experiment are summarized in Table 26.

In general, the lighter alkali metals are more soluble in the water and are easily removed from the sludge along with the nitrate. Some of the cesium (see ${ }^{137} \mathrm{Cs}$ recovery) appears to be bound to the sludge, which could be due to differences in ion exchange properties between the cesium and the lighter alkali metals. The incomplete removal of ${ }^{137} \mathrm{Cs}$ from the sludge with water, caustic, and low acid washing has been observed in past experiments with the ORNL sludge samples.

The actinide and lanthanide elements are not very soluble in water and for most ORNL caustic waste systems these elements are not significantly removed from the sludge by a water wash. The solubility behavior of the actinide and lanthanide elements in the OHF sludge follows no general trend and are less predictable than expected. This unpredictable behavior is illustrated by the uranium, thorium, ${ }^{152} \mathrm{Eu}$, and the gross alpha recoveries listed in Table 26,. The water solubility of the alkaline earth elements, represented by calcium and strontium in Table 26, are a function of both the $\mathrm{pH}$ and the carbonate concentration. The water solubility of the calcium ranges from $2 \%$ to about $17 \%$ for the OHF sludge samples and the strontium solubility ranges from $4 \%$ to about 14 
$\%$. The solubility behavior for the calcium and strontium represents what would also be expected for the ${ }^{90} \mathrm{Sr}$ activity. Most of the other major metals are usually insoluble in a water wash except for the chromium which is probably present as the chromate anion; most anionic species tend to be more soluble in water than the metals.

Table 26 Recovery of selected species in water leach of the OHF sludge

\begin{tabular}{|c|c|c|c|c|c|}
\hline \multirow{2}{*}{$\begin{array}{l}\text { Analytical } \\
\text { Measurement }\end{array}$} & \multicolumn{5}{|c|}{ \% Recovery in Water Leach of Sludge } \\
\hline & $\mathbf{T 1}$ & T2 & T3 & $\mathbf{T} 4$ & T9 \\
\hline \multicolumn{6}{|l|}{ Selected metals } \\
\hline $\mathrm{pH}$ of water wash & 9.6 & 9.6 & 11.5 & 10.4 & 9.3 \\
\hline $\mathrm{Al}$ & 2.3 & 2.5 & 1.1 & 12.0 & $<0.1$ \\
\hline $\mathrm{Ca}$ & 2.1 & 16.9 & 1.2 & 12.8 & 0.9 \\
\hline $\mathrm{Cr}$ & $<0.1$ & 16.8 & 25.5 & 12.5 & $<0.1$ \\
\hline $\mathrm{Fe}$ & 2.7 & 4.5 & 2.0 & 19.8 & $<0.1$ \\
\hline $\mathrm{K}$ & 93.5 & 93.0 & 49.2 & 99.5 & 92.4 \\
\hline $\mathrm{Mg}$ & 1.5 & 5.0 & 0.1 & $<0.1$ & 0.5 \\
\hline $\mathrm{Na}$ & 89.4 & 92.9 & 72.9 & 97.7 & 90.2 \\
\hline $\mathrm{Sr}$ & 4.2 & 3.5 & 1.8 & 13.7 & 1.6 \\
\hline Th & 6.3 & 7.2 & 8.0 & 35.0 & 0.4 \\
\hline $\mathrm{U}$ & 6.9 & 6.3 & 4.5 & 25.2 & 3.5 \\
\hline \multicolumn{6}{|c|}{ Selected radionuclides } \\
\hline Gross alpha & 6.3 & 12.8 & 8.0 & 21.7 & 0.1 \\
\hline Gross beta & 6.7 & 9.3 & 2.8 & 14.2 & 2.4 \\
\hline${ }^{60} \mathrm{Co}$ & 3.6 & 10.5 & 4.2 & 8.8 & 0.8 \\
\hline${ }^{137} \mathrm{Cs}$ & 30.8 & 71.4 & 6.25 & 73.5 & 42.3 \\
\hline${ }^{152} \mathrm{Eu}$ & 7.9 & 52.1 & 7.3 & 20.8 & 0.8 \\
\hline
\end{tabular}




\subsection{Estimates for Compliance with WIPP WAC. Rev. 5 for OHF Sludge}

The purpose of this section is to establish upper boundary estimates, based upon a 55-gal. drum shipping container, for several of the preliminary nuclear properties criteria and requirements for RH-TRU waste as specified in the WIPP WAC ${ }^{13}$, Revision 5. Specifically, this section will develop estimates for the ${ }^{239} \mathrm{Pu}$ Fissile Gram Equivalent (FGE), ${ }^{239} \mathrm{Pu}$ Equivalent Activity, and Thermal Power or decay heat limits per RH-TRU canister. The preliminary RH-TRU limits per waste canister for each of these nuclear criteria are listed as follows,

$\begin{array}{lll}- & { }^{239} \mathrm{Pu} \text { FGE } & <325 \mathrm{~g} \\ - & { }^{239} \mathrm{Pu} \text { Equivalent Activity } & <1000 \mathrm{Ci} \\ \text { - } & \text { Thermal Power } & <300 \text { watts. }\end{array}$

All of the estimates presented in this section are based on the data for the sludge samples collected from the center of each OHF tank.

\subsubsection{Estimates for ${ }^{239} \mathrm{Pu}$ Fissile Gram Equivalent in OHF Sludge}

For the OHF sludge, the ${ }^{239} \mathrm{Pu}$ FGE can be estimated by the summation of the gram-equivalents for ${ }^{233} \mathrm{U},{ }^{235} \mathrm{U}$, and ${ }^{239} \mathrm{Pu}$. As shown in Table 27, the ${ }^{233} \mathrm{U}$ and ${ }^{235} \mathrm{U}$ dominate the total ${ }^{239} \mathrm{Pu}$ FGE for the OHF sludge samples and the ${ }^{239} \mathrm{Pu}$ is less than $3 \%$ of the total fissile gram equivalent. Based on packaging the wet sludge in 55-gal. drums, none of the OHF sludge inventory would approach the RH-TRU limit of $325 \mathrm{~g}$ per canister for the ${ }^{239} \mathrm{Pu}$ FGE. Estimates for the total weight (Kg) of sludge taken from each OHF tank and placed in a 55 gal. drum are listed in Table 30.

Table 27 Estimates for ${ }^{239} \mathrm{Pu}$ FGE with the OHF sludge

\begin{tabular}{|c|c|c|c|c|c|c|}
\hline Isotope & $\begin{array}{l}{ }^{239} \mathbf{P u} \text { FGE } \\
\text { factor }\end{array}$ & $\begin{array}{c}\mathrm{T1} \\
(\mathrm{mg} / \mathrm{Kg})\end{array}$ & $\begin{array}{c}\mathrm{T} 2 \\
(\mathrm{mg} / \mathrm{Kg})\end{array}$ & $\begin{array}{c}\mathrm{T3} \\
(\mathrm{mg} / \mathrm{Kg})\end{array}$ & $\begin{array}{c}\mathrm{T} 4 \\
(\mathrm{mg} / \mathrm{Kg})\end{array}$ & $\begin{array}{c}\text { T9 } \\
\text { (mg/Kg) }\end{array}$ \\
\hline${ }^{233} \mathrm{U}$ & 0.865 & 22 & 22 & 43 & 69 & 18 \\
\hline $235 \mathrm{U}$ & 0.641 & 10 & 7.2 & 23 & 30 & 12 \\
\hline${ }^{239} \mathrm{Pu}$ & 1.000 & 2.4 & 2.4 & 1.4 & 2.8 & 2.4 \\
\hline \multicolumn{2}{|c|}{$39 \mathrm{P}$ FGE ( $\mathrm{mgKg})$} & 278 & 60.0 & & 817 & 257 \\
\hline \multicolumn{2}{|c|}{${ }^{339} \mathrm{PH}$ raE fin 55 gaA (g) } & 4,77 & $1 \%, 728$ & ? 14.6 & 20.6 & 162 \\
\hline
\end{tabular}




\subsubsection{Estimates for ${ }^{239} \mathrm{Pu}$ Equivalent Activity in OHF Sludge}

Estimates for the total ${ }^{239} \mathrm{Pu}$ equivalent activity (Ci) in a 55-gal. drum for each of the OHF sludge samples are listed in Table 28 . The ${ }^{239} \mathrm{Pu}$ equivalent activity is based on following calculation,

$$
{ }^{239} P u \text { Equivalent Activity }=\sum_{i=1} \frac{A_{t}}{F_{t}}
$$

where $A_{i}$ is the activity of radionuclide $i$, and $F_{i}$ is the ${ }^{239} \mathrm{Pu}$ equivalent activity weighting factor for radionuclide $i$. The weighting factors for the major radionuclides found in the OHF sludge are listed in Table 28. As shown in the last row of Table 28, all of the OHF sludge estimates for ${ }^{239} \mathrm{Pu}$ Equivalent activity would be less than $3 \mathrm{Ci}$ per $55 \mathrm{gal}$. drum, which is well below the RH-TRU limits. The $\mathrm{OHF}$ sludge is also below the $\mathrm{CH}$-TRU limit of $80 \mathrm{Ci}$ of plutonium equivalent activity for untreated waste in a 55-gal. drum and will not approach the $1000 \mathrm{Ci}$ WAC limit for a RH-TRU canister, which holds three 55-gal. drums.

Table 28 Estimates for ${ }^{239} \mathrm{Pu}$ equivalent activity with the OHF sludge

\begin{tabular}{|c|c|c|c|c|c|c|}
\hline Isotope & $\begin{array}{l}{ }^{239} \mathrm{Pu} \\
\text { wt. factor }\end{array}$ & $\begin{array}{c}\mathbf{T 1} \\
(\mathbf{B q} / \mathbf{g})\end{array}$ & $\begin{array}{r}\mathrm{T} 2 \\
(\mathrm{~Bq} / \mathrm{g})\end{array}$ & $\begin{array}{c}\text { T3 } \\
(\mathrm{Bq} / \mathrm{g})\end{array}$ & $\begin{array}{c}\mathrm{T} 4 \\
(\mathrm{~Bq} / \mathrm{g})\end{array}$ & $\begin{array}{c}\text { T9 } \\
\text { (Bq/g) }\end{array}$ \\
\hline${ }^{233} \mathrm{U}$ & 3.9 & 7900 & 7800 & 15000 & 24000 & 6400 \\
\hline${ }^{238} \mathrm{Pu}$ & 1.1 & 29000 & 22000 & 8900 & 20000 & 48000 \\
\hline${ }^{239} \mathrm{Pu}$ & 1.0 & 5500 & 5600 & 3300 & 6400 & 5500 \\
\hline${ }^{240} \mathrm{Pu}$ & 1.0 & 5300 & 6800 & 4700 & 8800 & 4500 \\
\hline${ }^{241} \mathrm{Pu}$ & 52.0 & 29000 & 50000 & 11000 & 29000 & 30000 \\
\hline${ }^{241} \mathrm{Am}$ & 1.0 & 52000 & 26000 & 15000 & 8000 & 13000 \\
\hline${ }^{244} \mathrm{Cm}$ & 1.9 & 350000 & 460000 & 250000 & 530000 & 270000 \\
\hline \multicolumn{2}{|c|}{ 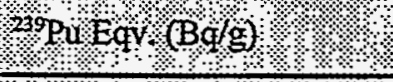 } & 275960 & 303470 & 166730 & 327040 & 210960 \\
\hline \multicolumn{2}{|c|}{${ }^{29} \mathrm{PuEqvin} 55$ gal (C) } & $1+2806$ & 227 & 1.23 & 223 & \%1, 13 \\
\hline
\end{tabular}

${ }^{a}$ Radionuclide-specific weighting factors for the ${ }^{239} \mathrm{Pu}$ equivalent activity taken from Appendix $\mathrm{A}$ of DOE/WIPP-069, Rev.5 


\subsubsection{Estimates of thermal power from decay heat in OHF sludge}

There is concern about the thermal power from the decay heat of the radionuclides present in waste packages prepared for WIPP disposal. These concerns are addressed in Revision 5 of the WIPP WAC, with limits of 40 watts for a TRUPACT-II container for CH-TRU waste and a limit of 300 watts for a RH-TRU canister. High decay heat is also an indicator for potential problems with hydrogen gas generation. The major radionuclides found in the OHF sludge are listed in Table 29 along with the "Q"-values" needed to calculate the decay heat for each isotope. The "Q"-values indicates the amount of energy (heat) that could be deposited in a radioactive material from each decay event if none of the radiation escaped from the material. The "Q"-value includes the sum of the average energies for different radiation types in $\mathrm{W} / \mathrm{Ci}$ (includes alpha and beta particles, discrete electrons, and photons, but not neutrinos).

An estimate of the decay heat distribution by radionuclide for the OHF sludge samples are listed in Table 30 along with an estimate for an upper boundary for total decay heat that would be in a 55 gal. drum full of wet sludge. These estimates indicate that the decay heat from OHF sludge is far below any of the WIPP WAC limits for thermal power and should have no impact on packaging requirements. For general interest, the relative percent distributions of the decay heat by radionuclide, beta activity, and alpha activity are listed in Table 31 . The distribution of decay heat as a function of OHF tank and radionuclide is illustrated in Fig. 8 for beta decay, and in Fig. 9 for alpha decay. It is interesting to note that the beta activity accounts for most of the decay heat output and that the heat from alpha decay is generally less than $15 \%$ of the total thermal power. 
Table 29 Isotopes that contribute to the decay heat in the OHF sludge

\begin{tabular}{|c|c|c|c|c|c|c|c|}
\hline Isotope & $\begin{array}{c}\text { "Q" value } \\
\text { (W/Ci) }\end{array}$ & $\begin{array}{l}\text { "Q" value } \\
\text { (W/Bq) }\end{array}$ & $\begin{array}{c}\mathrm{T} 1 \\
(\mathrm{~Bq} / \mathrm{g})\end{array}$ & $\begin{array}{c}\mathrm{T} 2 \\
(\mathrm{~Bq} / \mathrm{g})\end{array}$ & $\begin{array}{c}\mathrm{T} 3 \\
(\mathrm{~Bq} / \mathrm{g})\end{array}$ & $\begin{array}{c}T 4 \\
(\mathrm{Bg} / \mathrm{g})\end{array}$ & $\begin{array}{c}\mathrm{T} 9 \\
(\mathrm{~Bq} / \mathrm{g})\end{array}$ \\
\hline${ }^{\infty} \mathrm{Co}$ & $1.54 \mathrm{E}-02$ & $4.16 \mathrm{E}-13$ & $6.7 E+04$ & $7.7 E+04$ & $1.0 E+05$ & $1.6 \mathrm{E}+05$ & $4.9 E+04$ \\
\hline${ }^{s 0} \mathrm{Sr}$ & $1.16 E-03$ & $3.14 E-14$ & $2.0 E+07$ & $1.8 \mathrm{E}+07$ & $8.5 E+06$ & $1.6 E+07$ & $2.0 E+07$ \\
\hline soy & $5.54 \mathrm{E}-03$ & $1.50 \mathrm{E}-13$ & $2.0 E+07$ & $1.8 E+07$ & $8.5 E+06$ & $1.6 E+07$ & $2.0 E+07$ \\
\hline${ }^{137} \mathrm{Cs}$ & $1.01 E-03$ & $2.73 E-14$ & $3.9 \mathrm{E}+05$ & $3.5 E+05$ & $1.6 \mathrm{E}+06$ & $3.4 E+05$ & 2.6E+05 \\
\hline 137m $\mathrm{Ba}$ & $3.94 E-03$ & $1.06 E-13$ & 3.7E+05 & $3.3 E+05$ & $1.5 E+06$ & $3.2 E+05$ & $2.5 E+05$ \\
\hline${ }^{152} \mathrm{Eu}$ & $7.65 \mathrm{E}-03$ & $2.07 E-13$ & $6.3 E+04$ & $7.3 \mathrm{E}+04$ & $5.6 \mathrm{E}+04$ & $1.2 E+05$ & $4.3 E+04$ \\
\hline${ }^{154} \mathrm{Eu}$ & $9.08 \mathrm{E}-03$ & $2.45 \mathrm{E}-13$ & $4.3 E+04$ & $5.2 E+04$ & $3.4 E+04$ & $7.5 E+04$ & 3.1E+04 \\
\hline${ }^{155} \mathrm{Eu}$ & 7.59E-04 & $2.05 E-14$ & $0.0 E+00$ & $0.0 E+00$ & $5.9 \mathrm{E}+03$ & 1.1E+04 & $6.9 E+03$ \\
\hline${ }^{241} \mathrm{Pu}$ & 3.20E-05 & $8.65 \mathrm{E}-16$ & $2.9 E+04$ & $5.0 E+04$ & $1.1 E+04$ & $2.9 \mathrm{E}+04$ & $3.0 \mathrm{E}+04$ \\
\hline WOSa & (1) & 4 & 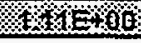 & . $9998=408$ & $6.495801 \%$ & $88 \% 932401=$ & 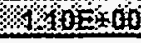 \\
\hline${ }^{223} \mathrm{U}$ & $2.86 E-02$ & $7.72 E-13$ & $7.9 E+03$ & $7.8 \mathrm{E}+03$ & $1.5 \mathrm{E}+04$ & $2.4 \mathrm{E}+04$ & $6.4 E+03$ \\
\hline${ }^{238} \mathrm{Pu}$ & $3.26 E-02$ & 8.81E-13 & $2.9 E+04$ & $2.2 E+04$ & $8.9 E+03$ & $2.0 E+04$ & $4.8 E+04$ \\
\hline${ }^{239} \mathrm{Pu}$ & $3.02 E-02$ & $8.17 E-13$ & $5.5 E+03$ & $5.6 \mathrm{E}+03$ & $3.3 E+03$ & $6.4 E+03$ & $5.5 E+03$ \\
\hline${ }^{240} \mathrm{Pu}$ & $3.06 \mathrm{E}-02$ & $8.26 E-13$ & $5.3 E+03$ & $6.8 \mathrm{E}+03$ & $4.7 E+03$ & $8.8 E+03$ & $4.5 E+03$ \\
\hline${ }^{241} \mathrm{Am}$ & 3.28E-02 & 8.87E-13 & $5.2 E+04$ & $2.6 \mathrm{E}+04$ & $1.5 E+04$ & $8.0 E+03$ & $1.3 E+04$ \\
\hline${ }^{24} \mathrm{Cm}$ & $3.44 E-02$ & $9.29 \mathrm{E}-13$ & $3.5 \mathrm{E}+05$ & $4.6 \mathrm{E}+05$ & $2.5 \mathrm{E}+05$ & $5.3 E+05$ & $2.7 E+05$ \\
\hline 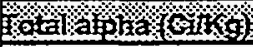 & (1:- & 2 & $2226: 02$ & 2843 Ex 22 & $80.028=03$ & 1.60 & $92392 \times 68$ \\
\hline Total beta in 55 gal. & um (Ci): & & 306.51 & 276.37 & 149.77 & 225.05 & 265.41 \\
\hline Total alpha in 550 & drum (Ci): & & 3.37 & 3.95 & 2.19 & 4.07 & 2.27 \\
\hline
\end{tabular}

Table 30 Distribution of decay heat in OHF sludge

\begin{tabular}{|c|c|c|c|c|c|c|c|}
\hline Isotope & $\begin{array}{c}\text { "Q" value } \\
\text { (N/Ci) }\end{array}$ & $\begin{array}{l}\text { "Q" value } \\
(\mathrm{w} / \mathrm{Bq})\end{array}$ & $\begin{array}{c}\text { T1 } \\
(w / K g)\end{array}$ & $\begin{array}{c}\text { T2 } \\
(w / K g)\end{array}$ & $\begin{array}{c}\text { T3 } \\
(W / \mathrm{Kg})\end{array}$ & $\begin{array}{c}\text { T4 } \\
(W / K g)\end{array}$ & $\begin{array}{c}\text { T9 } \\
\text { (W/Kg) }\end{array}$ \\
\hline${ }^{60} \mathrm{Co}$ & $1.54 E-02$ & $4.16 E-13$ & 2.79E-05 & $3.21 E-05$ & 4.16E-05 & $6.66 \mathrm{E}-05$ & 2.04E-05 \\
\hline${ }^{90} \mathrm{Sr}$ & 1.16E-03 & $3.14 E-14$ & $6.27 \mathrm{E}-04$ & $5.64 \mathrm{E}-04$ & $2.66 E-04$ & $5.02 E-04$ & $6.27 E-04$ \\
\hline soy & $5.54 E-03$ & $1.50 E-13$ & 2.99E-03 & $2.70 E-03$ & 1.27E-03 & $2.40 E-03$ & 2.99E-03 \\
\hline${ }^{137} \mathrm{Cs}$ & 1.01E-03 & 2.73E-14 & $1.06 \mathrm{E}-05$ & $9.55 E-06$ & 4.37E-05 & $9.28 E-06$ & 7.10E-06 \\
\hline${ }^{137 \mathrm{mBa}}$ & $3.94 \mathrm{E}-03$ & $1.06 E-13$ & $3.93 E-05$ & 3.53E-05 & 1.61E-04 & $3.43 E-05$ & 2.62E-05 \\
\hline${ }^{152} \mathrm{Eu}$ & 7.65E-03 & $2.07 E-13$ & $1.30 \mathrm{E}-05$ & $1.51 E-05$ & $1.16 E-05$ & $2.48 \mathrm{E}-05$ & 8.89E-06 \\
\hline${ }^{154} \mathrm{Eu}$ & $9.08 \mathrm{E}-03$ & $2.45 E-13$ & $1.06 \mathrm{E}-05$ & $1.28 E-05$ & 8.34E-06 & $1.84 \mathrm{E}-05$ & 7.61E-06 \\
\hline${ }^{155} \mathrm{Eu}$ & $7.59 E-04$ & $2.05 E-14$ & $0.00 E+00$ & $0.00 E+00$ & 1.21E-07 & $2.26 \mathrm{E}-07$ & $1.42 E-07$ \\
\hline${ }^{241} \mathrm{Pu}$ & $3.20 \mathrm{E}-05$ & $8.65 E-16$ & $2.51 \mathrm{E}-08$ & 4.32E-08 & $9.51 E-09$ & $2.51 E-08$ & 2.59E-08 \\
\hline${ }^{230} \mathrm{U}$ & $2.86 E-02$ & $7.72 \mathrm{E}-13$ & $6.10 \mathrm{E}-06$ & $6.02 E-06$ & 1.16E-05. & $1.85 \mathrm{E}-05$ & 4.94E-06 \\
\hline${ }^{238} \mathrm{Pu}$ & $3.26 E-02$ & $8.81 E-13$ & $2.55 E-05$ & $1.94 \mathrm{E}-05$ & $7.84 E-06$ & $1.76 E-05$ & 4.23E-05 \\
\hline${ }^{239} \mathrm{Pu}$ & $3.02 E-02$ & $8.17 E-13$ & $4.50 E-06$ & 4.58E-06 & 2.70E-06 & 5.23E-06 & 4.50E-06 \\
\hline${ }^{220} \mathrm{Pu}$ & $3.06 E-02$ & $8.26 E-13$ & $4.38 \mathrm{E}-06$ & 5.62E-06 & 3.88E-06 & $7.27 E-06$ & 3.72E-06 \\
\hline${ }^{244} \mathrm{Am}$ & $3.28 E-02$ & $8.87 E-13$ & 4.61E-05 & $2.31 E-05$ & $1.33 E-05$ & $7.10 E-06$ & 1.15E-05 \\
\hline${ }^{24} \mathrm{Cm}$ & $3.44 \mathrm{E}-02$ & $9.29 E-13$ & $3.25 \mathrm{E}-04$ & 4.27E- -04 & $2,32 \mathrm{E}-04$ & $4.92 E-04$ & 2.51E-04 \\
\hline 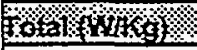 & $\approx$. & & Y O USE OS & $3,856=03$ & 2008403 & $3.6020 \% 43$ & 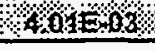 \\
\hline Density & $(\mathrm{Kg} / \mathrm{L}):$ & & 1.33 & 1.33 & 1.31 & 1.21 & 1.16 \\
\hline \multicolumn{3}{|c|}{ Total in 55 gal drum $(\mathrm{Kg})$ : } & 277 & 277 & 273 & 252 & 241 \\
\hline \multicolumn{3}{|c|}{ Total in 55 gal drum (watt): } & 1.145 & 1.066 & 0.566 & 0.907 & 0.968 \\
\hline
\end{tabular}


Table 31 Summary of relative decay heat in the OHF sludge

\begin{tabular}{|c|c|c|c|c|c|c|c|}
\hline Isotope & $\begin{array}{c}Q " \text { value } \\
\text { (W/Ci) }\end{array}$ & $\begin{array}{c}\text { "Q" value } \\
\text { (W/Bq) }\end{array}$ & $\begin{array}{c}\text { T1 } \\
(\% \text { Watt })\end{array}$ & $\begin{array}{c}\text { T2 } \\
\text { (\% Watt) }\end{array}$ & $\begin{array}{c}\text { T3 } \\
\text { (\% Watt) }\end{array}$ & $\begin{array}{c}\text { T4 } \\
\text { (\% Watt) }\end{array}$ & $\begin{array}{c}\text { T9 } \\
\text { (\% Watt) }\end{array}$ \\
\hline${ }^{60} \mathrm{Co}$ & $1.54 E-02$ & $4.16 \mathrm{E}-13$ & $0.67 \%$ & $0.83 \%$ & $2.00 \%$ & $1.85 \%$ & $0.51 \%$ \\
\hline${ }^{90} \mathrm{Sr}$ & $1.16 \mathrm{E}-03$ & $3.14 E-14$ & $15.16 \%$ & $14.66 \%$ & $12.83 \%$ & $13.94 \%$ & $15.64 \%$ \\
\hline soy & $5.54 \mathrm{E}-03$ & $1.50 E-13$ & $72.42 \%$ & $70.00 \%$ & $61.27 \%$ & $66.57 \%$ & $74.68 \%$ \\
\hline${ }^{137} \mathrm{Cs}$ & 1.01E-03 & $2.73 E-14$ & $0.26 \%$ & $0.25 \%$ & $2.10 \%$ & $0.26 \%$ & $0.18 \%$ \\
\hline${ }^{137 \mathrm{~m}} \mathrm{Ba}$ & $3.94 E-03$ & $1.06 \mathrm{E}-13$ & $0.95 \%$ & $0.92 \%$ & $7.76 \%$ & $0.95 \%$ & $0.65 \%$ \\
\hline${ }^{152} \mathrm{Eu}$ & 7.65E-03 & 2.07E-13 & $0.31 \%$ & $0.39 \%$ & $0.56 \%$ & $0.69 \%$ & $0.22 \%$ \\
\hline${ }^{15} \mathrm{EU}$ & $9.08 E-03$ & $2.45 E-13$ & $0.26 \%$ & $0.33 \%$ & $0.40 \%$ & $0.51 \%$ & $0.19 \%$ \\
\hline${ }^{155} \mathrm{Eu}$ & 7.59E-04 & 2.05E-14 & $0.00 \%$ & $0.00 \%$ & $0.01 \%$ & $0.01 \%$ & $0.00 \%$ \\
\hline${ }^{241} \mathrm{Pu}$ & 3.20 E-05 & 8.65E-16 & $0.00 \%$ & $0.00 \%$ & $0.00 \%$ & $0.00 \%$ & $0.00 \%$ \\
\hline \multicolumn{2}{|l|}{ Total beta heat (\%): } & & $90.04 \%$ & $87.38 \%$ & $86.93 \%$ & $84.77 \%$ & $92.07 \%$ \\
\hline $233 U$ & $2.86 E-02$ & $7.72 \mathrm{E}-13$ & $0.15 \%$ & $0.16 \%$ & $0.56 \%$ & $0.51 \%$ & $0.12 \%$ \\
\hline${ }^{238} \mathrm{Pu}$ & $3.26 \mathrm{E}-02$ & 8.81E-13 & $0.62 \%$ & $0.50 \%$ & $0.38 \%$ & $0.49 \%$ & $1.05 \%$ \\
\hline${ }^{239} \mathrm{Pu}$ & $3.02 E-02$ & 8.17E-13 & $0.11 \%$ & $0.12 \%$ & $0.13 \%$ & $0.15 \%$ & $0.11 \%$ \\
\hline${ }^{240} \mathrm{Pu}$ & 3.06E-02 & 8.26E-13 & $0.11 \%$ & $0.15 \%$ & $0.19 \%$ & $0.20 \%$ & $0.09 \%$ \\
\hline${ }^{241} \mathrm{Am}$ & $3.28 E-02$ & 8.87E-13 & $1.12 \%$ & $0.60 \%$ & $0.64 \%$ & $0.20 \%$ & $0.29 \%$ \\
\hline${ }^{244} \mathrm{Cm}$ & $3.44 \mathrm{E}-02$ & $9.29 E-13$ & $7.86 \%$ & $11.10 \%$ & $11.18 \%$ & $13.68 \%$ & $6.25 \%$ \\
\hline Total alpha heat ( & & & $9.96 \%$ & $12.62 \%$ & $13.07 \%$ & $15.23 \%$ & $7.93 \%$ \\
\hline
\end{tabular}


Figure 8 Distribution of Beta Decay Heat in OHF Sludge

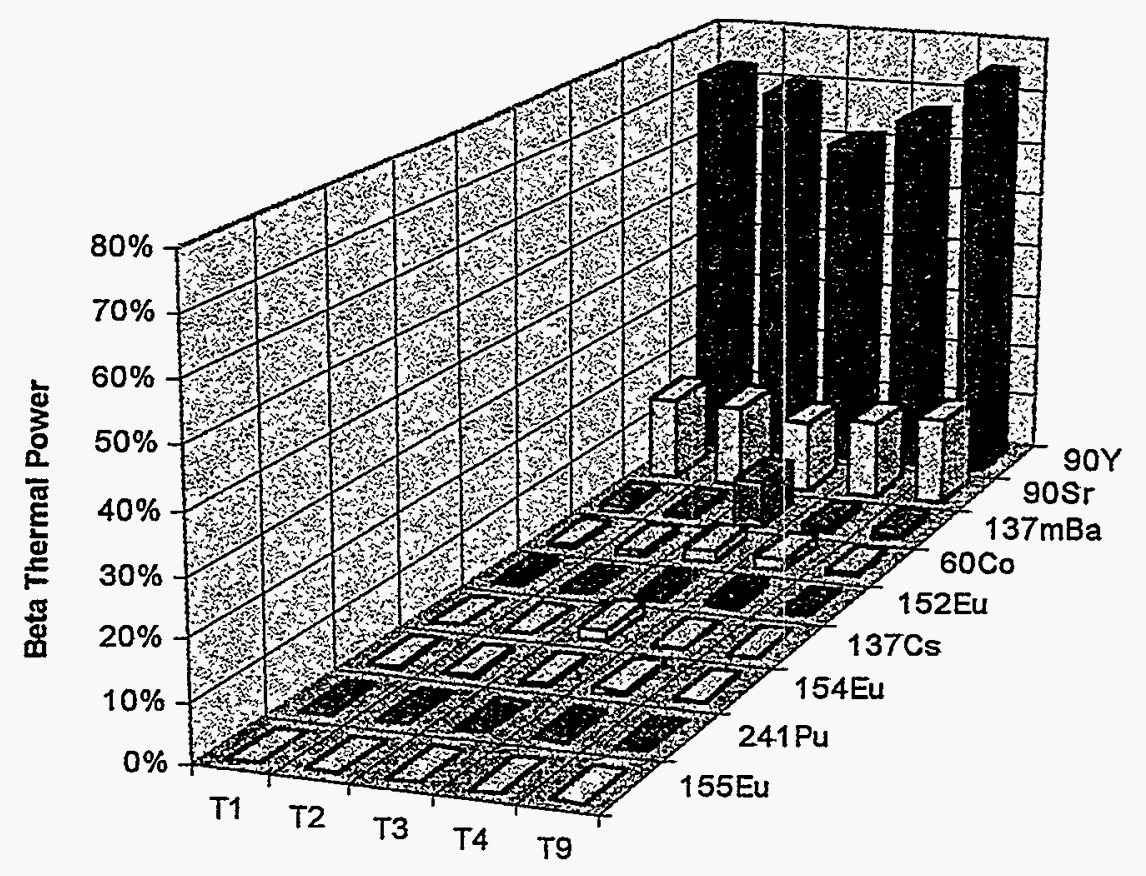

Figure 9 Distribution of Alpha Decay Heat in OHF Sludge

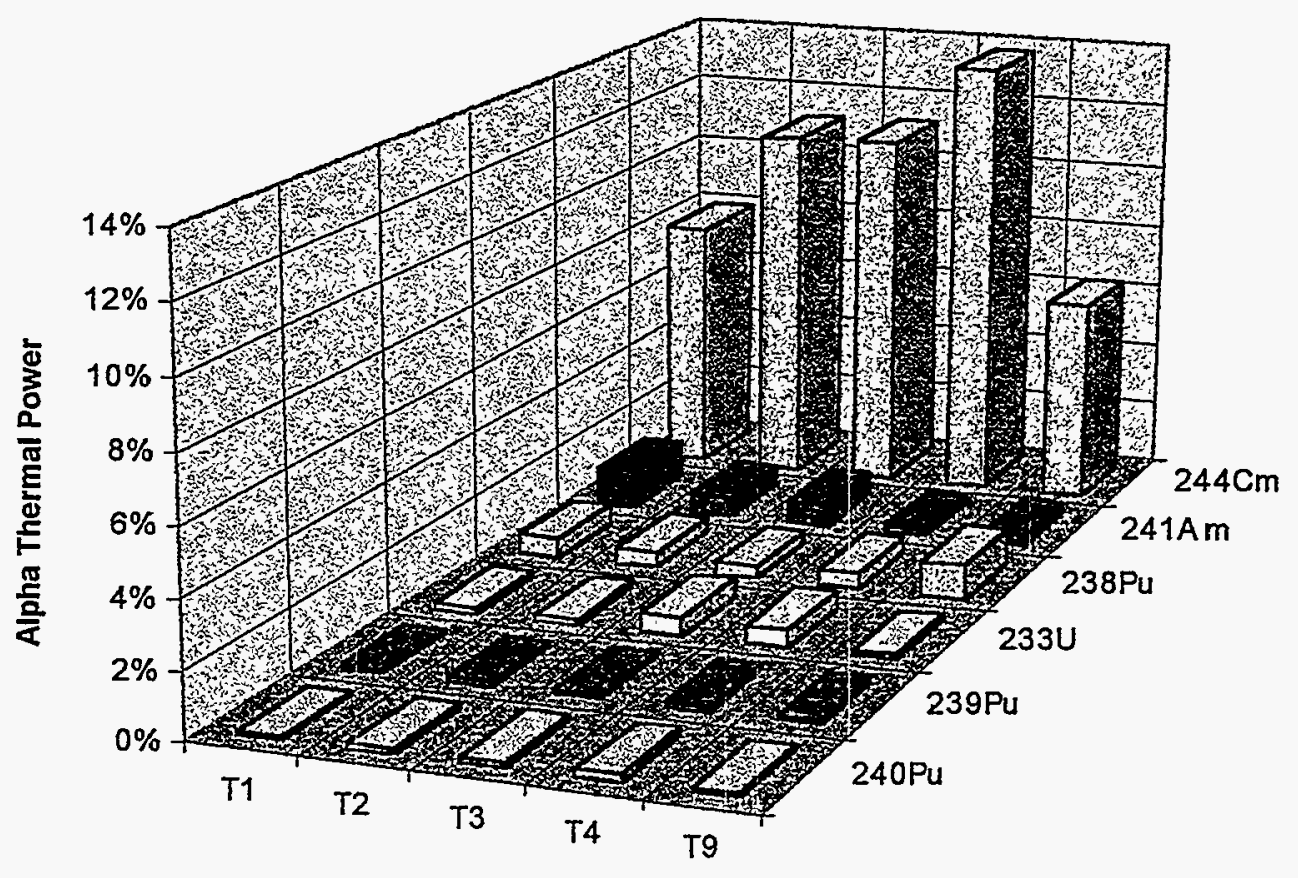




\subsection{Summary of Organic Analytical Results}

The organic target compound list (TCL) hits and the tentatively identified compounds (TIC) from the GC-MS, GC-FID, and GC-ECD analyses are listed in Table 32 for the supernatant samples and Table 33 for the sludge samples. These tables for the liquid and sludge samples also include the regulatory limits based on the Universal Treatment Standards (UTS) from the Land Disposal Restrictions (LDR) regulations (40 CFR 268.48). The RCRA TCLP limits for organic compounds are listed in Table 34 and are reproduced for each corresponding RCRA compounds in parenthesis next to the compound name for quick reference in the liquid and sludge result tables. For the organic chemical characterization results the following reporting conventions are used:

Reporting limits

B

D

$\mathbf{E}$

$\mathbf{J}$

$\mathbf{U}$

TIC
The reporting limits are the concentrations above which the response of the instrument for the calibrated range of concentrations is linear.

Data qualifier meaning that the compound was also found in the accompanying laboratory blank sample.

Data qualifier meaning sample dilution was required.

Data qualifier indicating that the reported concentration of the compound exceeded the calibration range of the instrument.

Data qualifier meaning that the compound was estimated at a concentration below the reporting limit; also used to indicate that the concentrations for tentatively identified compounds (TICs) are estimates.

Data qualifier meaning compound was not detected or was below the method detection limits (MDL) and the MDL corrected for dilution was reported.

Tentatively identified compound. The identification is based upon mass spectral data only, and the quantitation is based upon the response factor of the nearest eluting internal standard. All TIC values are estimates and are flagged with the " $J$ " qualifier. 
Table 32 Analytical organic data for OHF liquid samples

\begin{tabular}{|c|c|c|c|c|c|c|}
\hline \multirow{2}{*}{ Target Compound } & \multirow{2}{*}{$\begin{array}{c}\text { Reg. } \\
\text { Limit' } \\
\text { (mg/L) }\end{array}$} & \multicolumn{5}{|c|}{ Concentration in Liquid, mg/L } \\
\hline & & $\mathbf{T 1}$ & $\mathbf{T 2}$ & T3 & $\mathbf{T 4}$ & T9 \\
\hline \multicolumn{7}{|c|}{ Non halogenated Yolatile organic compound (NH vo A) } \\
\hline 67-64-1 Acetone & 0.28 & $2.0 \mathrm{U}$ & $2.0 \mathrm{U}$ & $3.3 \mathrm{~J}$ & $5.9 \mathrm{~J}$ & $2.0 \mathrm{U}$ \\
\hline 71-36-3 Butanol & 5.6 & $2.0 \mathrm{U}$ & $2.0 \mathrm{U}$ & 28. J & $3.0 \mathrm{~J}$ & $2.0 \mathrm{U}$ \\
\hline 78-83-1 Isobutanol & 5.6 & $2.0 \mathrm{U}$ & $2.0 \mathrm{U}$ & $2.0 \mathrm{U}$ & $2.0 \mathrm{U}$ & $2.0 \mathrm{U}$ \\
\hline 67-56-1 Methanol & 5.6 & $2.0 \mathrm{U}$ & $2.0 \mathrm{U}$ & 20. J & 22. J & $2.0 \mathrm{U}$ \\
\hline $\begin{array}{ll}\text { 78-93-3 Methyl ethyl ketone } & \text { (200) } \\
\text { 2-Butanone }\end{array}$ & 0.28 & $2.0 \mathrm{U}$ & $2.0 \mathrm{U}$ & $2.0 \mathrm{U}$ & $2.0 \mathrm{U}$ & $2.0 \mathrm{U}$ \\
\hline $110-86-1$ Pyridine & 0.014 & $2.0 \mathrm{U}$ & $2.0 \mathrm{U}$ & $2.0 \mathrm{U}$ & $2.0 \mathrm{U}$ & $2.0 \mathrm{U}$ \\
\hline \multicolumn{7}{|l|}{ Vonatileorganic Compounds (YOA) } \\
\hline 71-43-2 Benzene & 0.14 & $0.005 \mathrm{U}$ & $0.005 \mathrm{U}$ & $0.005 \mathrm{U}$ & $0.005 \mathrm{U}$ & $0.005 \mathrm{U}$ \\
\hline 75-25-2 Bromoform & 0.63 & $0.005 \mathrm{U}$ & $0.005 \mathrm{U}$ & $0.005 \mathrm{U}$ & $0.005 \mathrm{U}$ & $0.005 \mathrm{U}$ \\
\hline 75-15-0 Carbon disulfide & 3.8 & $0.005 \mathrm{U}$ & $0.005 \mathrm{U}$ & $0.005 \mathrm{U}$ & $0.005 \mathrm{U}$ & $0.005 \mathrm{U}$ \\
\hline 56-23-5 Carbon tetrachloride $\quad(0.5)$ & 0.057 & $0.005 \mathrm{U}$ & $0.005 \mathrm{U}$ & $0.005 \mathrm{U}$ & $0.005 \mathrm{U}$ & $0.005 \mathrm{U}$ \\
\hline 108-90-7 Chlorobenzene $\quad(100)$ & 0.057 & $0.005 \mathrm{U}$ & $0.005 \mathrm{U}$ & $0.005 \mathrm{U}$ & $0.005 \mathrm{U}$ & $0.005 \mathrm{U}$ \\
\hline 67-66-3 Chloroform & 0.046 & $0.063 \mathrm{~J}$ & $0.44 \mathrm{~J}$ & $0.005 \mathrm{U}$ & $0.047 \mathrm{~J}$ & $0.065 \mathrm{~J}$ \\
\hline 95-50-1 1,2-Dichlorobenzene & 0.088 & $0.005 \mathrm{U}$ & $0.005 \mathrm{U}$ & $0.005 \mathrm{U}$ & $0.005 \mathrm{U}$ & $0.005 \mathrm{U}$ \\
\hline 106-46-7 1,4-Dichlorobenzene $\quad(7.5)$ & 0.090 & $0.005 \mathrm{U}$ & $0.005 \mathrm{U}$ & $0.005 \mathrm{U}$ & $0.005 \mathrm{U}$ & $0.005 \mathrm{U}$ \\
\hline 107-06-2 1,2-Dichloroethane $\quad(0.5)$ & 0.21 & $0.005 \mathrm{U}$ & $0.005 \mathrm{U}$ & $0.005 \mathrm{U}$ & $0.005 \mathrm{U}$ & $0.005 \mathrm{U}$ \\
\hline 75-35-4 1,1-Dichloroethylene & 0.025 & $0.013 \mathrm{JB}$ & $0.005 \mathrm{U}$ & $0.005 \mathrm{U}$ & $0.005 \mathrm{U}$ & $0.006 \mathrm{JB}$ \\
\hline 100-41-4 Ethyl benzene & 0.057 & $0.005 \mathrm{U}$ & $0.005 \mathrm{U}$ & $0.005 \mathrm{U}$ & $0.005 \mathrm{U}$ & $0.005 \mathrm{U}$ \\
\hline 60-29-7. Ethyl ether & 0.12 & $0.005 \mathrm{U}$ & $0.005 \mathrm{U}$ & $0.005 \mathrm{U}$ & $0.005 \mathrm{U}$ & $0.005 \mathrm{U}$ \\
\hline 75-09-2 Methylene Chloride & 0.089 & $0.005 \mathrm{U}$ & $0.005 \mathrm{U}$ & $0.005 \mathrm{U}$ & $0.005 \mathrm{U}$ & $0.005 \mathrm{U}$ \\
\hline 79-34-5 1,1,2,2-Tetrachloroethane & 0.057 & $0.005 \mathrm{U}$ & $0.005 \mathrm{U}$ & $0.005 \mathrm{U}$ & $0.005 \mathrm{U}$ & $0.005 \mathrm{U}$ \\
\hline 127-18-4 Tetrachloroethylene (0.7) & 0.056 & $0.005 \mathrm{U}$ & $0.005 \mathrm{U}$ & $0.005 \mathrm{U}$ & $0.005 \mathrm{U}$ & $0.005 \mathrm{U}$ \\
\hline 108-88-3 Toluene & 0.080 & $0.005 \mathrm{U}$ & $0.005 \mathrm{U}$ & $0.005 \mathrm{U}$ & $0.006 \mathrm{~J}$ & $0.005 \mathrm{U}$ \\
\hline 71-55-6 1,1,1-Trichloroethane & 0.054 & $0.012 \mathrm{JB}$ & $0.005 \mathrm{U}$ & $0.006 \mathrm{JB}$ & $0.013 \mathrm{JB}$ & $0.005 \mathrm{U}$ \\
\hline 79-00-5 1,1,2-Trichloroethane & 0.054 & $0.005 \mathrm{U}$ & $0.005 \mathrm{U}$ & $0.005 \mathrm{U}$ & $0.005 \mathrm{U}$ & $0.005 \mathrm{U}$ \\
\hline 79-01-6 Trichloroethylene $\quad(0.5)$ & 0.054 & $0.005 \mathrm{U}$ & $0.005 \mathrm{U}$ & $0.005 \mathrm{U}$ & $0.005 \mathrm{U}$ & $0.005 \mathrm{U}$ \\
\hline 75-69-4 Trichlorofluoromethane & 0.020 & $0.005 \mathrm{U}$ & $0.005 \mathrm{U}$ & $0.005 \mathrm{U}$ & $0.005 \mathrm{U}$ & $0.005 \mathrm{U}$ \\
\hline $\begin{array}{l}\text { 76-13-1 1,1,2-Trichloro-1,2,2- } \\
\text { trifluoroethane }\end{array}$ & 0.057 & $0.005 \mathrm{U}$ & $0.005 \mathrm{U}$ & $0.005 \mathrm{U}$ & $0.005 \mathrm{U}$ & $0.005 \mathrm{U}$ \\
\hline 75-01-4 Vinyl Chloride & 0.27 & $0.005 \mathrm{U}$ & $0.005 \mathrm{U}$ & $0.005 \mathrm{U}$ & $0.005 \mathrm{U}$ & $0.005 \mathrm{U}$ \\
\hline
\end{tabular}




\begin{tabular}{|c|c|c|c|c|c|c|}
\hline \multirow{2}{*}{ Target Compound } & \multirow{2}{*}{$\begin{array}{l}\text { Reg. } \\
\text { Limit } \\
\text { (mg/L) }\end{array}$} & \multicolumn{5}{|c|}{ Concentration in Liquid, $\mathrm{mg} / \mathrm{L}$} \\
\hline & & T1 & $\mathbf{T}$ & T3 & $\mathbf{T 4}$ & T9 \\
\hline 1330-20-7 m\&p-Xylene & 0.32 & $0.005 \mathrm{U}$ & $0.005 \mathrm{U}$ & $0.005 \mathrm{U}$ & $0.006 \mathrm{~J}$ & $0.005 \mathrm{U}$ \\
\hline 95-47-6 o-Xylene & 0.32 & $0.005 \mathrm{U}$ & $0.005 \mathrm{U}$ & $0.005 \mathrm{U}$ & $0.005 \mathrm{U}$ & $0.005 \mathrm{U}$ \\
\hline \multicolumn{7}{|c|}{ 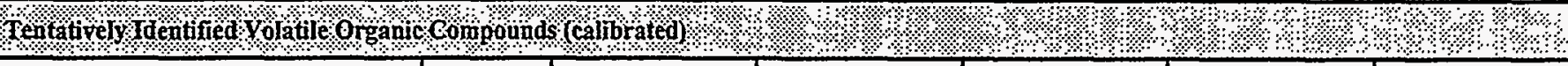 } \\
\hline 75-27-4 Bromodichloromethane & 0.35 & $0.005 \mathrm{U}$ & $0.005 \mathrm{U}$ & $0.005 \mathrm{U}$ & $0.005 \mathrm{U}$ & $0.005 \mathrm{U}$ \\
\hline 75-00-3 Chloroethane & 0.27 & $0.005 \mathrm{U}$ & $0.005 \mathrm{U}$ & $0.005 \mathrm{U}$ & $0.005 U$ & $0.005 \mathrm{U}$ \\
\hline 124-48-1 Dibromochloromethane & 0.057 & $0.005 \mathrm{U}$ & $0.005 \mathrm{U}$ & $0.005 \mathrm{U}$ & $0.005 \mathrm{U}$ & $0.005 \mathrm{U}$ \\
\hline 541-73-1 1,3-Dichlorobenzene & 0.036 & $0.005 \mathrm{U}$ & $0.005 \mathrm{U}$ & $0.005 \mathrm{U}$ & $0.005 \mathrm{U}$ & $0.005 \mathrm{U}$ \\
\hline 75-34-3 1,1-Dichloroethane & 0.059 & $0.005 \mathrm{U}$ & $0.005 \mathrm{U}$ & $0.005 \mathrm{U}$ & $0.005 \mathrm{U}$ & $0.005 \mathrm{U}$ \\
\hline 156-59-2 cis-1,2-Dichloroethylene & - & $0.005 \mathrm{U}$ & $0.01 \mathrm{~J}$ & $0.005 \mathrm{U}$ & $0.005 \mathrm{U}$ & $0.005 \mathrm{U}$ \\
\hline $\begin{array}{l}\text { 156-60-5 trans-1,2- } \\
\text { Dichloroethylene }\end{array}$ & 0.054 & $0.005 \mathrm{U}$ & $0.005 \mathrm{U}$ & $0.005 \mathrm{U}$ & $0.005 \mathrm{U}$ & $0.005 \mathrm{U}$ \\
\hline 78-87-5 1,2-Dichloropropane & 0.85 & $0.005 \mathrm{U}$ & $0.005 \mathrm{U}$ & $0.005 \mathrm{U}$ & $0.005 \mathrm{U}$ & $0.005 \mathrm{U}$ \\
\hline $\begin{array}{l}\text { 10061-02-6 trans-1,3- } \\
\text { Dichloropropylene }\end{array}$ & 0.036 & $0.005 \mathrm{U}$ & $0.005 U$ & $0.005 \mathrm{U}$ & $0.005 \mathrm{U}$ & $0.005 \mathrm{U}$ \\
\hline $\begin{array}{l}\text { 10061-01-5 cis-1,3- } \\
\text { Dichloropropylene }\end{array}$ & 0.036 & $0.005 \mathrm{U}$ & $0.005 U$ & $0.005 \mathrm{U}$ & $0.005 \mathrm{U}$ & $0.005 \mathrm{U}$ \\
\hline $\begin{aligned} \text { 108-10-1 } & \text { 4-Methyl-2-pentanone } \\
& \text { Methyl isobutyl ketone }\end{aligned}$ & 0.14 & $0.005 \mathrm{U}$ & $0.005 \mathrm{U}$ & $0.012 \mathrm{~J}$ & $0.027 \mathrm{~J}$ & $0.005 \mathrm{U}$ \\
\hline 100-42-5 Styrene & - & $0.005 \mathrm{U}$ & $0.005 \mathrm{U}$ & $0.005 \mathrm{U}$ & $0.005 \mathrm{U}$ & $0.005 \mathrm{U}$ \\
\hline 108-05-4 Vinyl Acetate & - & $0.005 \mathrm{U}$ & $0.005 \mathrm{U}$ & $0.005 \mathrm{U}$ & $0.005 \mathrm{U}$ & $0.005 \mathrm{U}$ \\
\hline \multicolumn{7}{|c|}{ Tentafiyely ldentifed Yolatile Organic Conpounds (uncalibrated) } \\
\hline 67-64-1 Acetone & 0.28 & $0.065 \mathrm{~J}$ & $0.005 \mathrm{U}$ & $1.3 \mathrm{JE}$ & $1.1 \mathrm{JE}$ & $0.013 \mathrm{~J}$ \\
\hline 74-83-9 Bromomethane & 0.11 & $0.023 \mathrm{~J}$ & $0.005 \mathrm{U}$ & $0.057 \mathrm{JB}$ & $0.041 \mathrm{JB}$ & $0.032 \mathrm{JB}$ \\
\hline $\begin{array}{ll}\text { 78-93-3 } & \begin{array}{l}\text { Methyl ethyl ketone } \\
\text { 2-Butanone }\end{array} \\
\end{array}$ & 0.28 & $0.009 \mathrm{~J}$ & $0.005 \mathrm{U}$ & $0.31 \mathrm{JE}$ & $0.51 \mathrm{JE}$ & $0.005 \mathrm{U}$ \\
\hline 74-87-3 Chloromethane & 0.19 & $0.005 \mathrm{U}$ & $0.005 \mathrm{U}$ & $0.005 \mathrm{U}$ & $0.005 \mathrm{U}$ & $0.005 \mathrm{U}$ \\
\hline 110-43-0 2-Heptanone & - & $0.005 \mathrm{U}$ & - & - & $0.066 \mathrm{~J}$ & - \\
\hline 591-78-6 2-Hexanone & - & $0.005 \mathrm{U}$ & $0.005 \mathrm{U}$ & $0.013 \mathrm{~J}$ & $0.075 \mathrm{~J}$ & $0.005 \mathrm{U}$ \\
\hline $\begin{array}{ll}590-50-1 & \begin{array}{l}2-P e n t a n o n e, 4,4- \\
\text { dimethyl- }\end{array} \\
\end{array}$ & - & $0.005 \mathrm{U}$ & - & $0.039 \mathrm{~J}$ & $0.086 \mathrm{~J}$ & - \\
\hline 115-11-7 1-Propene, 2-methyl- & - & - & - & - & - & $0.045 \mathrm{~J}$ \\
\hline Unknown & - & $0.7 \mathrm{JB}$ & $0.8 \mathrm{JB}$ & $0.08 \mathrm{JB}$ & $0.06 \mathrm{JB}$ & $0.6 \mathrm{JB}$ \\
\hline Unknown & - & $0.2 \mathrm{~J}(8)^{\mathrm{b}}$ & $0.3 \mathrm{~J}(3)^{\mathrm{b}}$ & $1.5 \mathrm{~J}(17)^{\mathrm{b}}$ & $1.8 \mathrm{~J}(16)^{b}$ & $0.3 \mathrm{~J}(5)^{6}$ \\
\hline
\end{tabular}




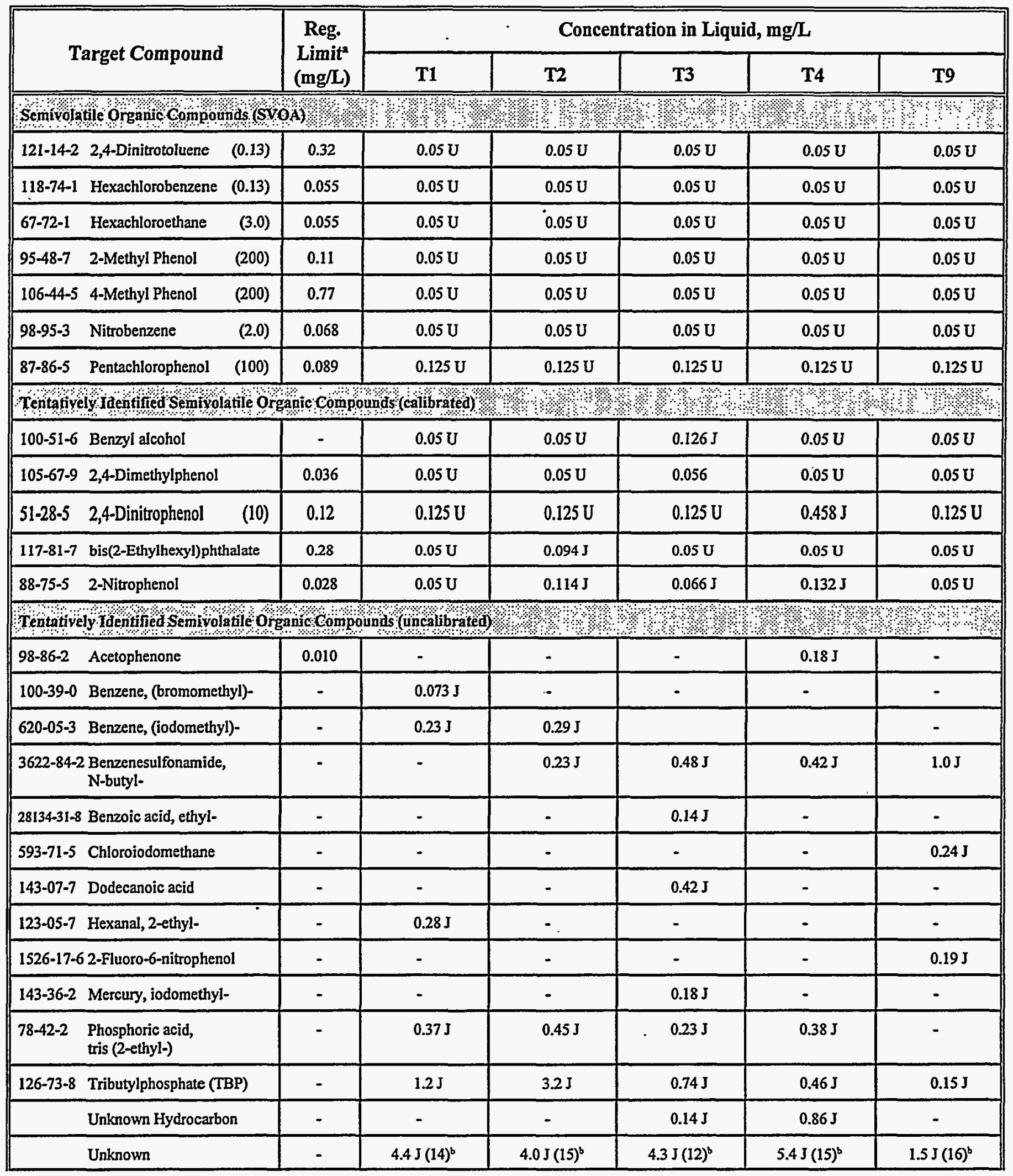




\begin{tabular}{|c|c|c|c|c|c|c|c|}
\hline \multirow{2}{*}{\multicolumn{2}{|c|}{ Target Compound }} & \multirow{2}{*}{$\begin{array}{l}\text { Reg. } \\
\text { Limit } \\
\text { (mg/L) }\end{array}$} & \multicolumn{5}{|c|}{ Concentration in Liquid, mg/L } \\
\hline & & & $\mathbf{T 1}$ & $\mathbf{T 2}$ & T3 & T4 & T9 \\
\hline $12674-11-2$ & Aroclor-1016 & 2 & $0.01 \mathrm{U}$ & $0.01 \mathrm{U}$ & $0.01 \mathrm{U}$ & $0.01 \mathrm{U}$ & $0.01 \mathrm{U}$ \\
\hline $11141-16-5$ & Aroclor-1232 & 2 & $0.01 \mathrm{U}$ & $0.01 \mathrm{U}$ & $0.01 \mathrm{U}$ & $0.01 \mathrm{U}$ & $0.01 \mathrm{U}$ \\
\hline $53469-21-9$ & Aroclor-1242 & 2 & $0.01 \mathrm{U}$ & $0.01 \mathrm{U}$ & $0.01 \mathrm{U}$ & $0.01 \mathrm{U}$ & $0.01 \mathrm{U}$ \\
\hline $12672-29-6$ & Aroclor-1248 & 2 & $0.01 \mathrm{U}$ & $0.01 \mathrm{U}$ & $0.01 \mathrm{U}$ & $0.01 \mathrm{U}$ & $0.01 \mathrm{U}$ \\
\hline $11097-69-1$ & Aroclor-1254 & 2 & $0.01 \mathrm{U}$ & $0.01 \mathrm{U}$ & $0.01 \mathrm{U}$ & $0.01 \mathrm{U}$ & $0.01 \mathrm{U}$ \\
\hline
\end{tabular}

Regulatory limits based on Universal Treatment Standards (Wastewater standard) from 40 CFR \$268.48.

${ }^{b}$ Number of compounds grouped together listed in parenthesis. 
Table 33 Analytical organic data for OHF sludge samples

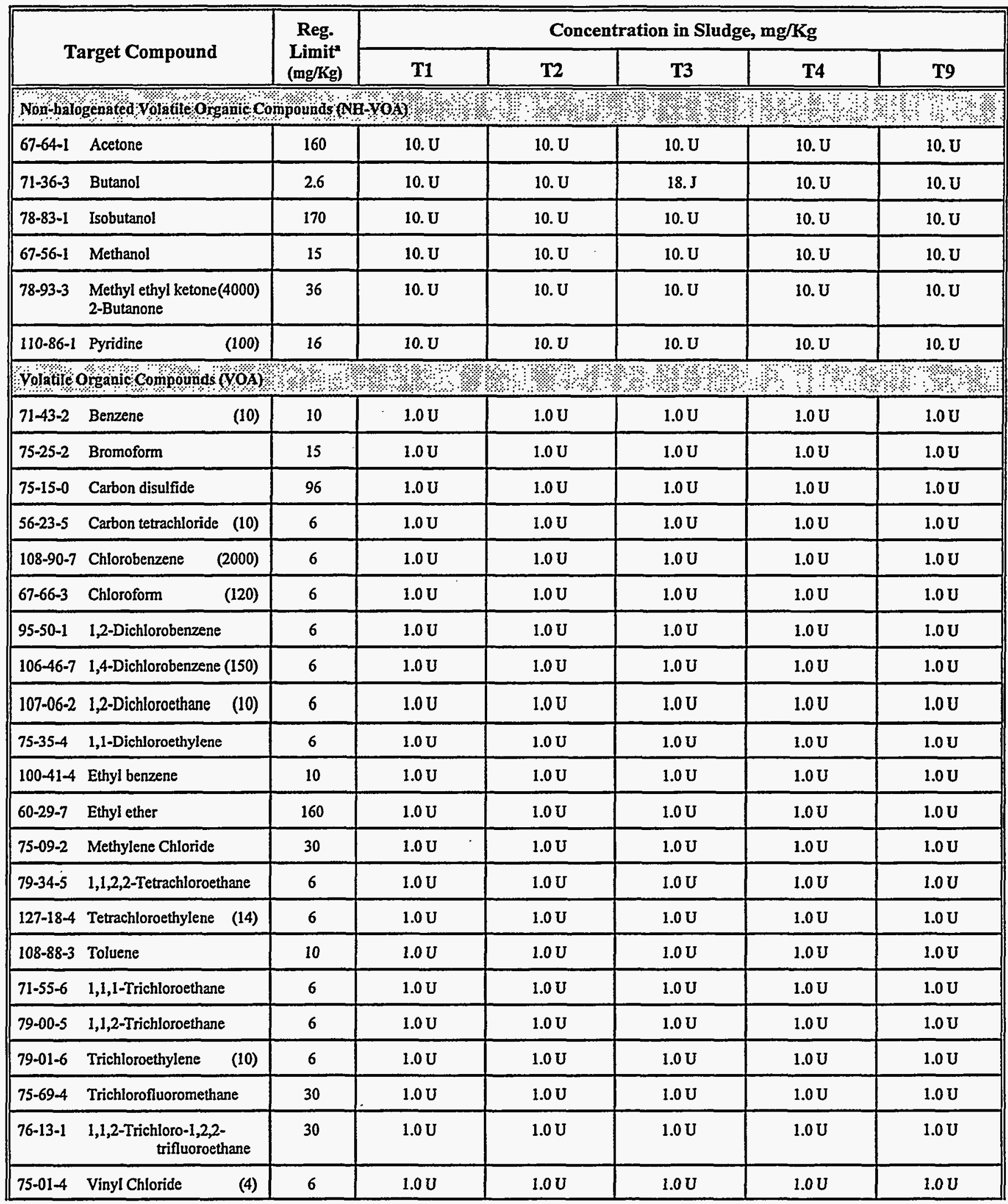




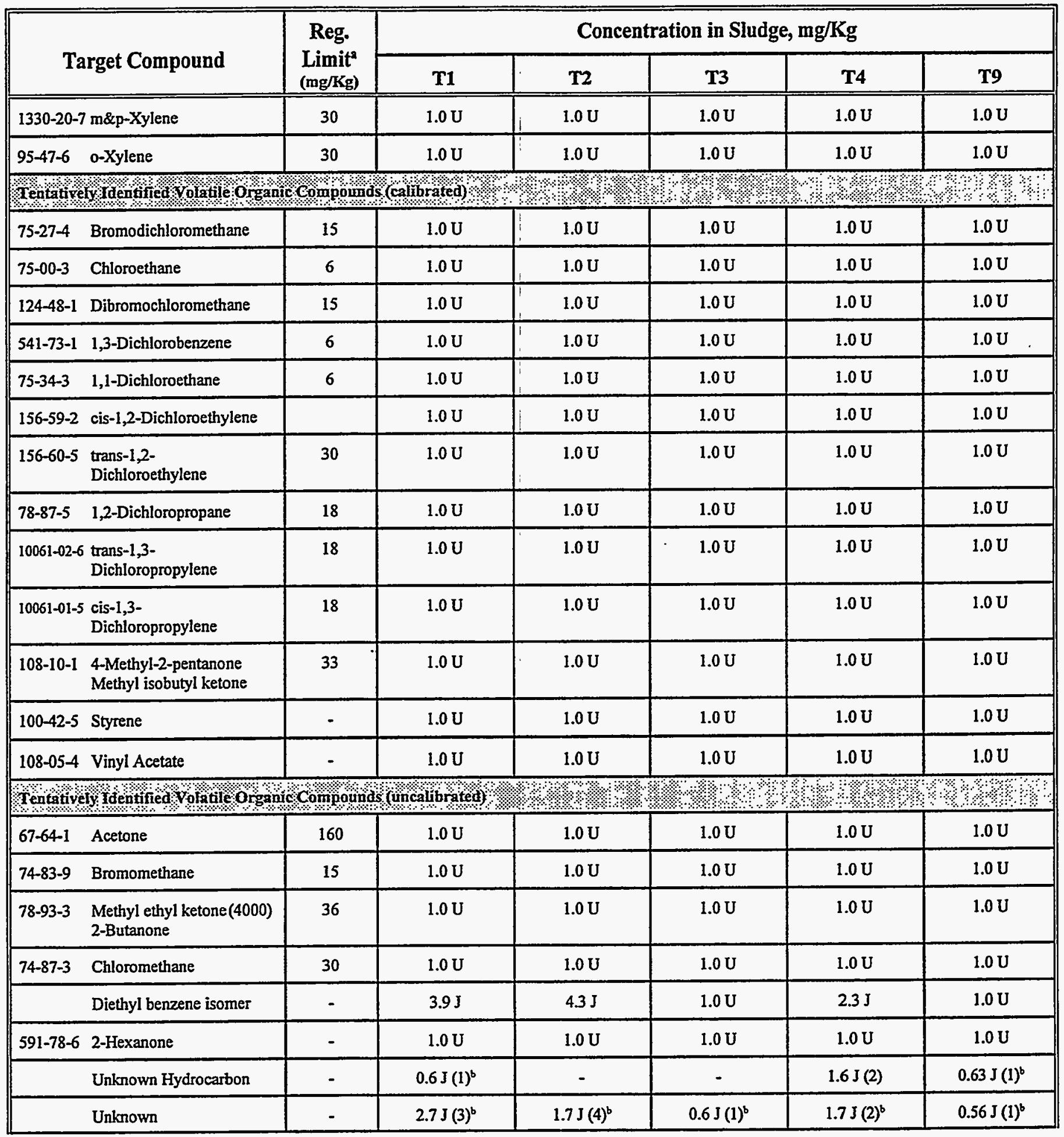




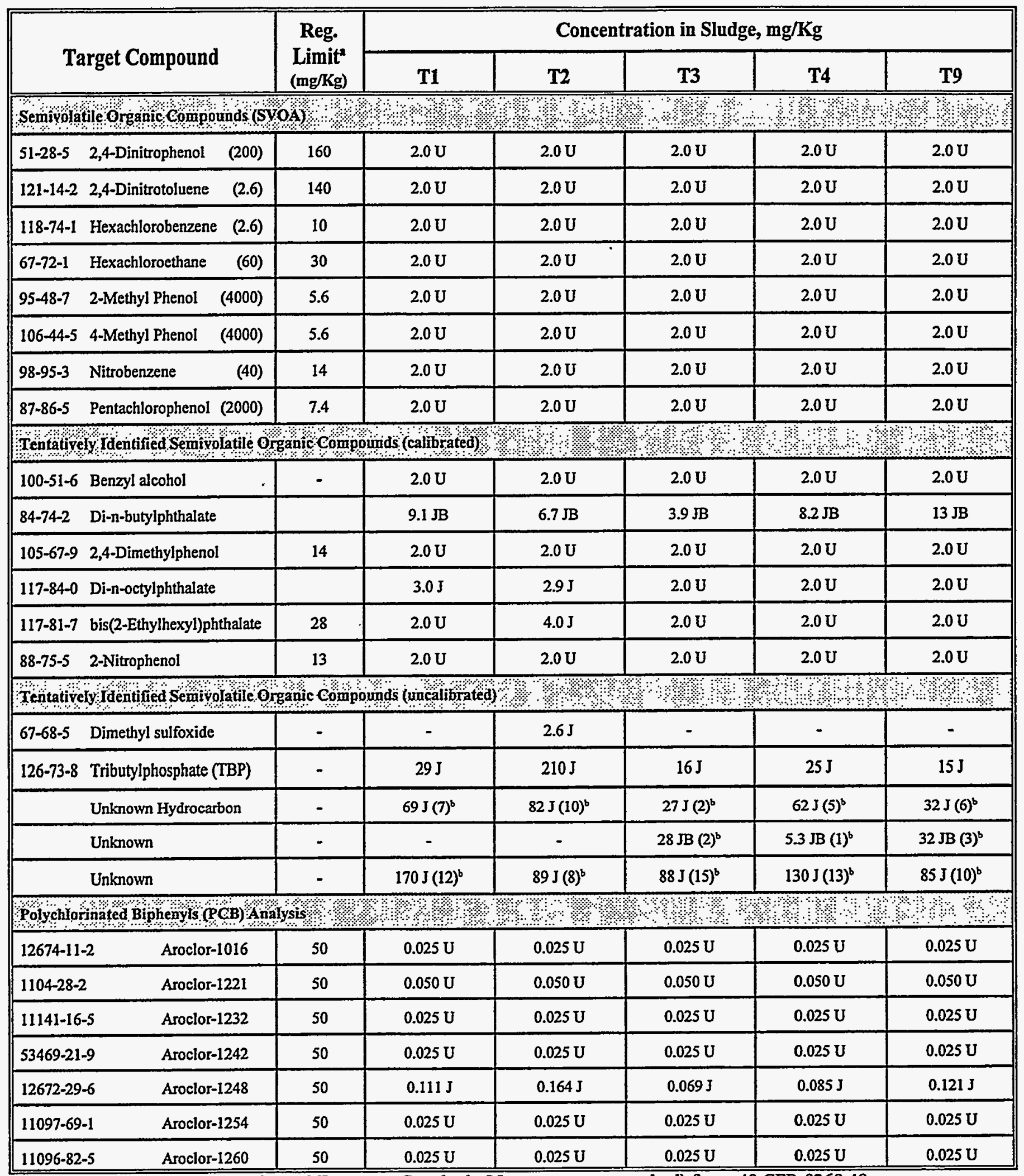

Regulatory limits based on Universal Treatment Standards (Nonwastewater standard) from 40 CFR \$268.48.

b Number of compounds grouped together listed in parenthesis. 
Table 34 RCRA TCLP limits for target organic compounds

\begin{tabular}{|c|c|c|c|}
\hline CAS No. & Target Compound & $\begin{array}{c}\text { TCLP Limit } \\
\text { (mg/L) }\end{array}$ & $\begin{array}{c}\text { Sludge Limit } \\
\text { (mg/Kg) }\end{array}$ \\
\hline $71-43-2$ & Benzene & 0.5 & 10.0 \\
\hline $56-23-5$ & Carbon tetrachloride & 0.5 & 10.0 \\
\hline $108-90-7$ & Chlorobenzene & 100.0 & 2000.0 \\
\hline $67-66-3$ & Chloroform & 6.0 & 120.0 \\
\hline $95-48-7$ & o-Cresol & 200.0 & 4000.0 \\
\hline $108-39-4$ & m-Cresol & 200.0 & 4000.0 \\
\hline $106-44-5$ & p-Cresol & 200.0 & 4000.0 \\
\hline $51-28-5$ & 2,4-Dinitrophenol & 10.0 & 200.0 \\
\hline $106-46-7$ & 1,4-Dichlorobenzene & 7.5 & 150.0 \\
\hline $107-06-2$ & 1,2-Dichloroethane & 0.5 & 10.0 \\
\hline $75-35-4$ & 1,1-Dichloroethylene & 0.7 & 14.0 \\
\hline $121-14-2$ & 2,4-Dinitrotoluene & 0.13 & 2.6 \\
\hline $118-74-1$ & Hexachlorobenzene & 0.13 & 2.6 \\
\hline $87-68-3$ & Hexachloro-1,3-butadiene & 0.5 & 10.0 \\
\hline $67-72-1$ & Hexachloroethane & 3.0 & 60.0 \\
\hline 78-93-3 & Methyl ethyl ketone & 200.0 & 4000.0 \\
\hline $98-95-3$ & Nitrobenzene & 2.0 & 40.0 \\
\hline $87-86-5$ & Pentachlorophenol & 100.0 & 2000.0 \\
\hline $110-86-1$ & Pyridine & 5.0 & 100.0 \\
\hline $127-18-4$ & Tetrachloroethylene & 0.7 & 14.0 \\
\hline $79-01-6$ & Trichloroethylene & 0.5 & 10.0 \\
\hline $95-95-4$ & 2,4,5-Trichlorophenol & 400.0 & 8000.0 \\
\hline $88-06-2$ & 2,4,6-Trichlorophenol & 2.0 & 40.0 \\
\hline $75-01-4$ & Vinyl chloride & 0.2 & 4.0 \\
\hline
\end{tabular}

${ }^{2}$ Regulatory limits taken from SW-846, Chapter 7, Table 7-1 (also see 40 CFR §261.24).

${ }^{b}$ The sludge limit is the concentration required in a solid sample to exceed the limit after a TCLP leach. 


\subsection{Discussion of Organic Analysis}

The concentration of non-polar organic compounds identified in the OHF samples was limited to a few organic compounds observed above the detection limits in the supernatant samples and only a few hundred $\mathrm{mg} / \mathrm{Kg}$ observed in the sludge samples. The organic compounds identified included products from the Purex and other actinide separations used by past nuclear processing operations within ORNL

Much higher levels of polar organic acids were observed in the T3 and T4 supernatant samples by ion chromatography (see Tables 5 and 6). The basic (deionized) form of several common organic acids were observed in these liquid samples including formate, acetate, and oxalate ranging from 200 $-900 \mathrm{mg} / \mathrm{L}$. The corresponding sludge samples from T3 and T4 also had elevated levels of formate, acetate, and oxalate ranging from $50-1100 \mathrm{mg} / \mathrm{Kg}$ (see Tables 8-12). The sludge samples from $\mathrm{T} 1$, $\mathrm{T} 2$, and $\mathrm{T} 9$ had trace levels of formate and oxalate present.

Heavy emulsification was noted during the SVOC and PCBs methylene chloride extraction preparations for the T3 liquid sample, which was probably due to water entrained in the solvent. The T3 sample was treated with sodium sulfate to absorb the water and clean up the organic phase prior to the SVOC and PCB analysis. The acid extractable surrogates were not recovered in the supernatant extractions due to the highly alkaline conditions from the sample matrix. This recovery problem with surrogates is common for a caustic sample matrix. For the remaining OHF liquid samples there was no significant problems encountered during the organic measurements.

The identification of the total organic content in radioactive waste tanks continues to be a problem for this laboratory and others throughout the DOE sites. Similar to past experiences, the sum of the identified organic compounds is considerably lower than what the total organic carbon content would indicate. The partial balance of the total organic carbon content was most likely due to the presence of high molecular weight polar compounds which would not have been detected by the gas and liquid chromatographic methods used for the organic measurements. Also, most forms of biological organic matter, including decay products from vegetation and microorganisms, would not be detected by the conventional measurement techniques used for waste characterization. The 
biological organic material would contribute to the final concentration reported from the total organic carbon measurements.

\subsection{Regulatory Concerns due to Organic Compounds in OHF Waste}

The regulatory definition states that the waste exhibits the characteristic of toxicity (RCRA) if the TCLP extract from a sub-sample of the waste contains any of the compounds listed in Table 34 at a concentration greater than or equal to the respective values given in the table. If the waste contains $<0.5 \%$ filterable solids, the liquid (supernatant for this project) itself, after filtering, is considered to be the extract for the purpose of analysis. None of the OHF liquid or sludge samples exceeded the TCLP leachate limits listed in Table 34.

The regulatory limits listed in Table 32 and 33 are requirements from the Land Disposal Restrictions (LDR) program (40 CFR §268.41). These limits are provided for information only and can be found under Universal Treatment Standards (UTS) in 40 CFR §268.48, Table UTS. Although some of the organic compounds in the liquid phase exceed the UTS limits, this should not be a problem for the disposal of radioactive waste. In general, the regulatory driver for both TRU and non-TRU radioactive waste is the WIPP Target Compound List which includes the RCRA compounds, some common organic solvents, plus a few additional organic compounds of interest to EPA and New Mexico state regulators. The WIPP waste acceptance criteria also requires the concentration of any PCBs present in the waste be documented. The waste acceptance criteria for the Nevada Test Site (NTS), which only accepts non-TRU waste, only requires that the generator demonstrate that the waste is not RCRA and does not contain PCBs over $50 \mathrm{ppm}$. The NTS requirements are similar to most other private and state operated disposal sites for non-TRU radioactive waste. Therefore, if the generator uses the WIPP target compound list for guidance and includes PCB data, all the organic results required for disposal of most radioactive waste should be covered. Most generators of radioactive waste use process knowledge to exclude organic complexing agents or chelators from the waste data package. 


\subsection{Summary of Rheometry Measurements}

\subsection{Rheometry Tests}

Some basic principles and definitions concerning rheometry measurements on sludge and slurry samples is provided in Appendix $\mathrm{C}$ for the reader's convenience. The rheometry data presented in this section was collected for the purpose of obtaining a low cost, simple, and rapid estimate of flow properties for the OHF sludges. The sludge samples used for the rheometry tests were a composite of the north and south end core samples from each respective tank. The sludge from each tank was combined and sonicated to collect the needed amount of material and to obtain a homogeneous mixture prior to any rheometry test being performed. It was necessary to mix the sludges samples prior to the shear strength tests even though the mixing resulted in measurements with lower shear strength values. Ideally the tests would have been performed on sludge samples that were left undisturbed as much as possible, but a number of factors had to be addressed that precluded performing the tests on undisturbed sludges. First, the amount of material in a single sampling core from the OHF tanks was typically not enough to fully cover the shear vane as required for the tests and when there was enough material to cover the vane there was still not enough to meet the distance requirements needed between the vane and container walls. Secondly, when the material was transferred from the sampler to the tests containers large air gaps would form as the sludge wrapped around itself generating air pockets. The shear vane could penetrate these air pockets and it could not be ensured that the vane was completely in contact with the sample as needed to perform a valid measurement.

Earlier experiments were performed to compare the shear strength measurements on a sludge sample before and after mixing. The shear strength measurements were collected on a BVEST sludge sample with minimal prior mixing, followed by measurements after the sludge sample was well mixed (sonicated) and allowed to settle for 48 hours. The conditions for the second shear strength measurement on the BVEST sample was similar to the measurements on the OHF sludge. The shear strength value determined after the sludge was sonicated was approximately half of the value measured on the unmixed sludge sample. This change in shear strength may be unacceptable but the qualified results are sufficient for the simple rheometry tests requested this project. For the reasons discussed above, the shear strength measurements should be considered to be good estimates (within 
a order of magnitude) for a sludge sample collected with a core device. If more accuracy is needed for the shear strength measurements it would be necessary to perform in-situ field measurements on each tank sludge.

An additional comment noted on the rheometry measurements included the observation that small coarse particulate remained visible in the sonicated mixtures. These small particles can degrade the rheometry measurements and are believed to be responsible for some of the noise observed in some of the data curves. The final interpretation of the rheometry data presented in this section is left to the user of this document. No data reduction was performed nor were any mathematical corrections or curve fitting/smoothing (such as the power law equation) applied to the data. The data is presented as measured directly from the instrument with the parameters listed in this document. All of the data presented in this report is available from the RMAL for those readers who desire more detailed information or wish to do additional analysis of the data. In addition to the rheometry data collected, all the sample handling steps performed during the rheometry tests were documented on video. The video documents the physical appearance of the sludges and their visible flow characteristics. Included on the video are all sample transfers from the field sample collection tubes, all sample mixing, dilutions, and rheometry tests.

Shear strength and viscosity data were collected for each of the OHF sludges utilizing a Rotovisco RV30 Searle type rotational CR (controlled rate) rheometer, available from Gebrueder Haake ${ }^{\circledR}$ $\mathrm{GmbH}$, Karlsruhe or Haake (USA). The Searle type measuring system is comprised of a calibrated spring whose deflection is proportional to the torque and converted by a transducer to an electronic signal. The system is close to friction free and provides an instantaneous response. Two types of sensors were used for the tests. A Haake FL100 shear vane rotor was used to determine shear strengths. This rotor is comprised of six vanes that are $16 \mathrm{~mm}$ high and has a diameter of $22 \mathrm{~mm}$. Viscosity and flow curves were generated using an immersion system comprised of an immersion tube and a modified Haake MV DIN rotor. The modified rotor has a smaller diameter than the original ( $36.4 \mathrm{~mm}$ vs. $38.7 \mathrm{~mm}$ ) and a height of $58.08 \mathrm{~mm}$. 
All tests were conducted in a hot cell due to the highly radioactive nature of the samples. The sensors and measuring system were located in the cell and connected to a control unit outside of the cell via a communication cable. A personal computer connected to the control unit was used to operate the rheometer software, set test parameters, and collect data. The shear strength measurements were performed at the ambient temperature found in the hot cell. The temperature for each viscosity measurement was maintained constant during each experiment utilizing a plexiglass bath located in the hot cell with cooling coils that were supplied from a temperature-controlled water bath located outside of the hot cell.

\subsection{Shear Strength Measurements}

The shear strength of a liquid or slurry provides a measure of the shear conditions needed to overcome the fluid's resistance to flow. It is measured using a constant shear rate and determined from the peak shear stress on a shear stress versus time curve. In theory, a sludge begins as an elastic solid with a specific shear strength. Once this shear strength is surpassed the sludge, if it demonstrates thixotropic properties (the potential to reform a gel structure), becomes a thixotropic fluid that with sufficient time to reach steady state flow conditions may exhibit a yield stress. A yield stress was not observed in the OHF sludge samples with the FL100 shear vane rotor employed. After discussions ${ }^{15}$ with staff from Pacific Northwest Laboratories (PNL) that are experienced with rheometry measurements on DOE waste tank sludge samples, it was decided that a yield stress probably can not be measured on the concentrated sludge suspension and therefore would not be observed in the OHF measurements. Also, discussions with the Haake technical staff indicates that an improvement in the shear strength data would be obtained if a larger shear vane rotor was used for the measurements. The data obtained with the FL100 rotor was less than $1 \%$ of the full scale for the recommended operating range and is subject to large errors.

As stated earlier the sludge samples used for the rheometry tests were composites of the north and south core samples from each respective tank and each composite was sonicated to ensure a homogeneous mixture prior to any rheometry tests being performed. The sonicated mixtures were allowed to stand for forty eight hours prior to the shear strength determinations in an attempt to allow the gel structure of the composite to reform. Studies have not been done to determine the 
optimum time required for the sludge to reform after mixing. Based on the study of less complicated fluids, the magnitude of the shear strength can be a function of the time interval a sludge is allowed to set (return to a gel structure) before measurements are performed. It is important to remember that the laboratory conditions do not match the conditions in found in a waste tank. The shear strength measurements performed in the laboratory do not represent what would be observed in a tank where the sludge has formed layers and has been left undisturbed for long periods of time. An in-situ measurement at varying depths would yield more accurate shear strength measurements for in-tank conditions. For the lab measurements, a distance of at least three times the diameter of the shear vane was maintained between the shear vane and the sample container walls to ensure that there was no influence from the walls of the container during the tests. The shear tests were performed at ambient temperatures within the hot cells and actual temperatures are documented on each shear strength plot.

For the shear strength measurements, the FL100 six vane rotor was rotated at a speed to allow the vanes to cut through the sludge at a rate of one sixth of a revolution in a two minute period $(0.083$ $\mathrm{rpm})$. This allowed for sufficient data to be collected before the elasticity of the sludge was broken. Measurements were obtained at two depths. For the first measurement the shear vane was submerged into the sample so that the vane was completely covered (noted on the graphs as "Vane just below surface") by the sample. When the first test was complete the shear vane was then submerged deeper into the sample to a depth of approximately 1.5 inches from the top of the shear vane to the surface of the sample (noted on the graphs as "Vane submerged approx. 1.5 inches"). This second depth allowed for comparison of shear strength measurements at two different sample depths and still maintained the requirement that the vane be greater than three times it's diameter from the walls of the container. Only one depth measurement was obtained on tank sludge T-9 due to instrument problems. Because of the restricted view into the hot cell the submergence depths of the shear vane were estimated. Precautions were taken to ensure that the shear vane rotor was completely covered and the proper vane to wall clearance was maintained. None of the measurements were corrected for the vane submergence depth. 
Figure 10 Shear Strength Curves for T1 Sludge Composite

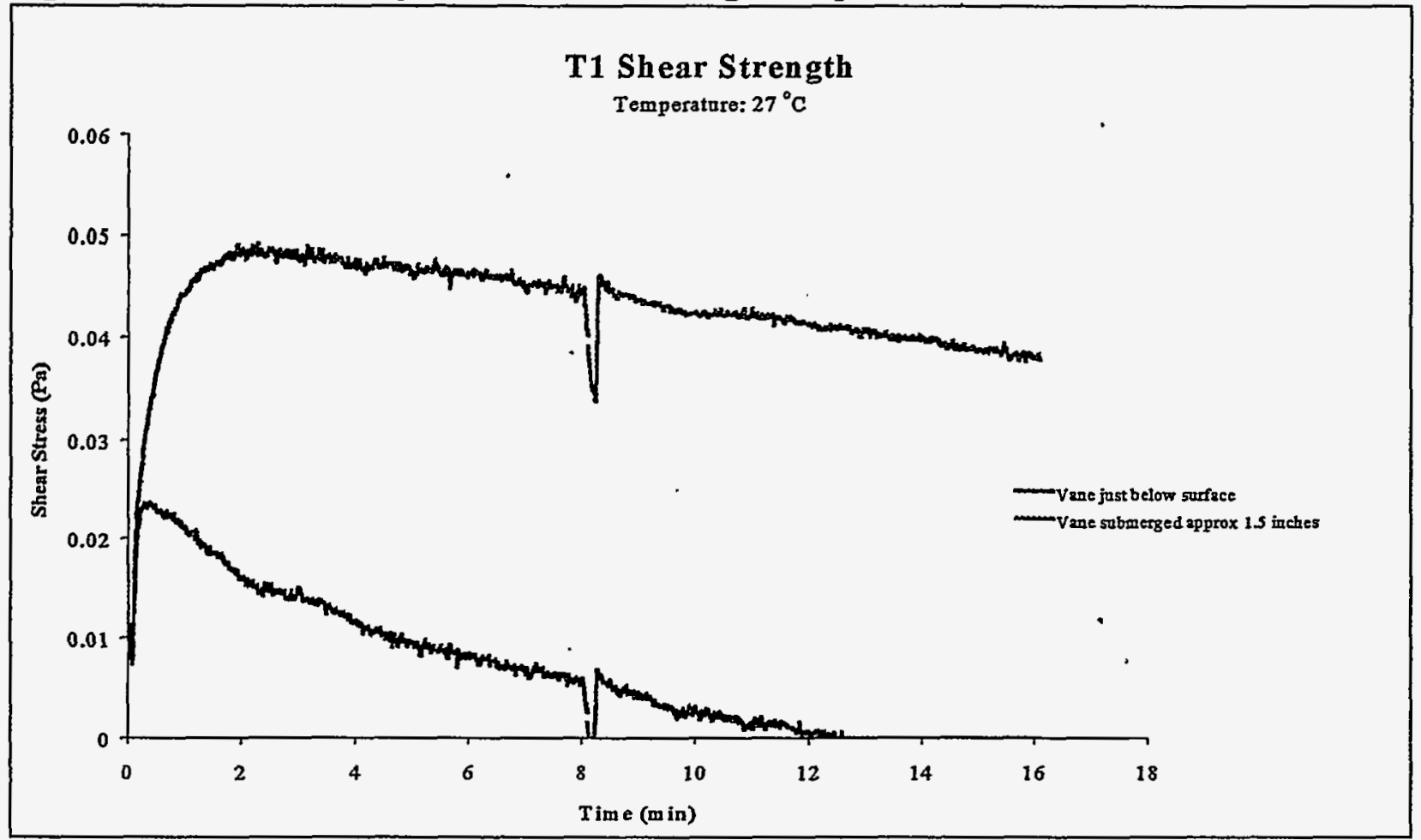

Figure 11 Shear Strength Curves for T2 Sludge Composite

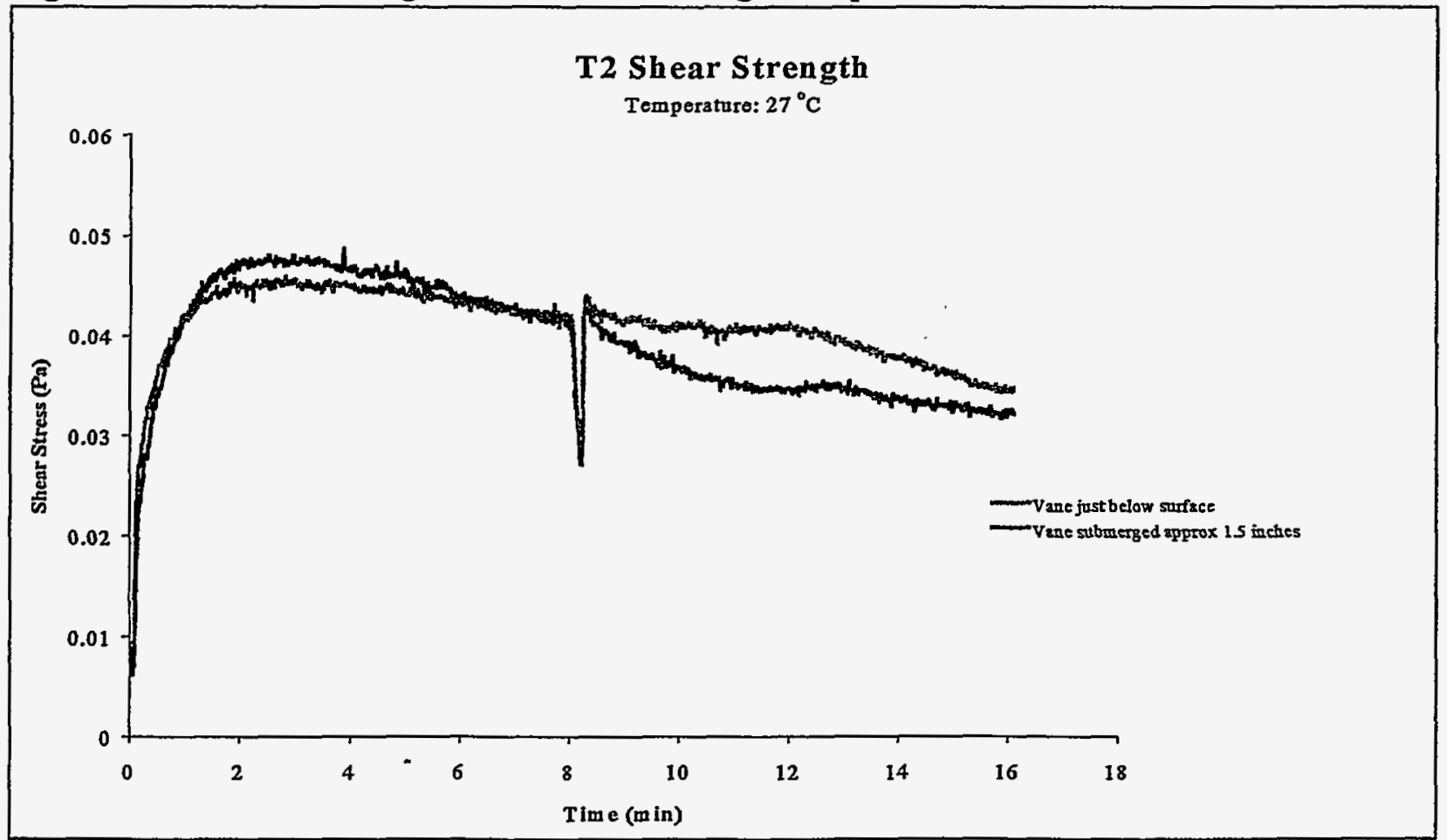


Figure 12 Shear Strength Curves for T3 Sludge Composite

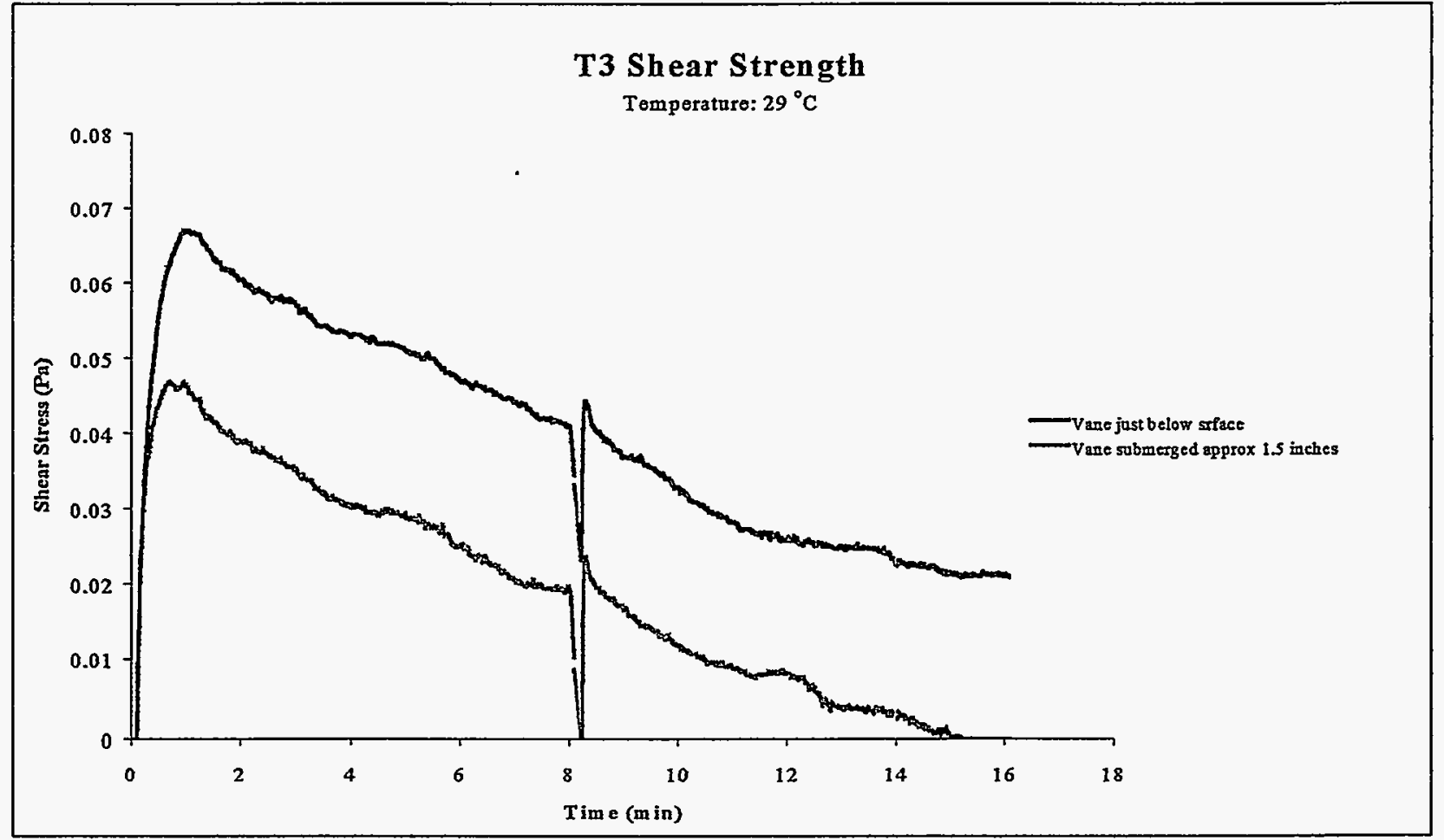

Figure 13 Shear Strength Curves for T4 Sludge Composite

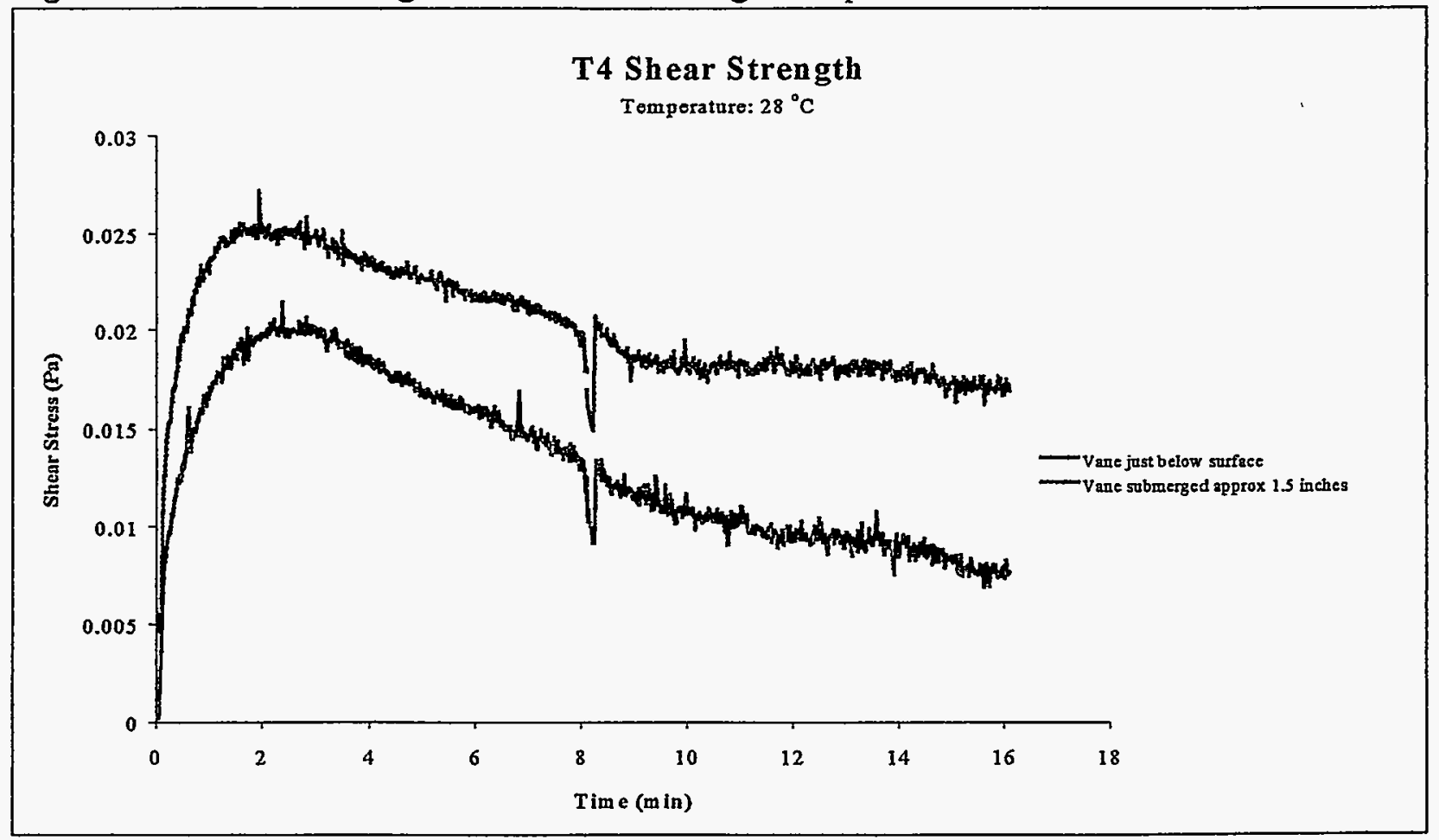


Figure 14 Shear Strength Curves for T9 Sludge Composite

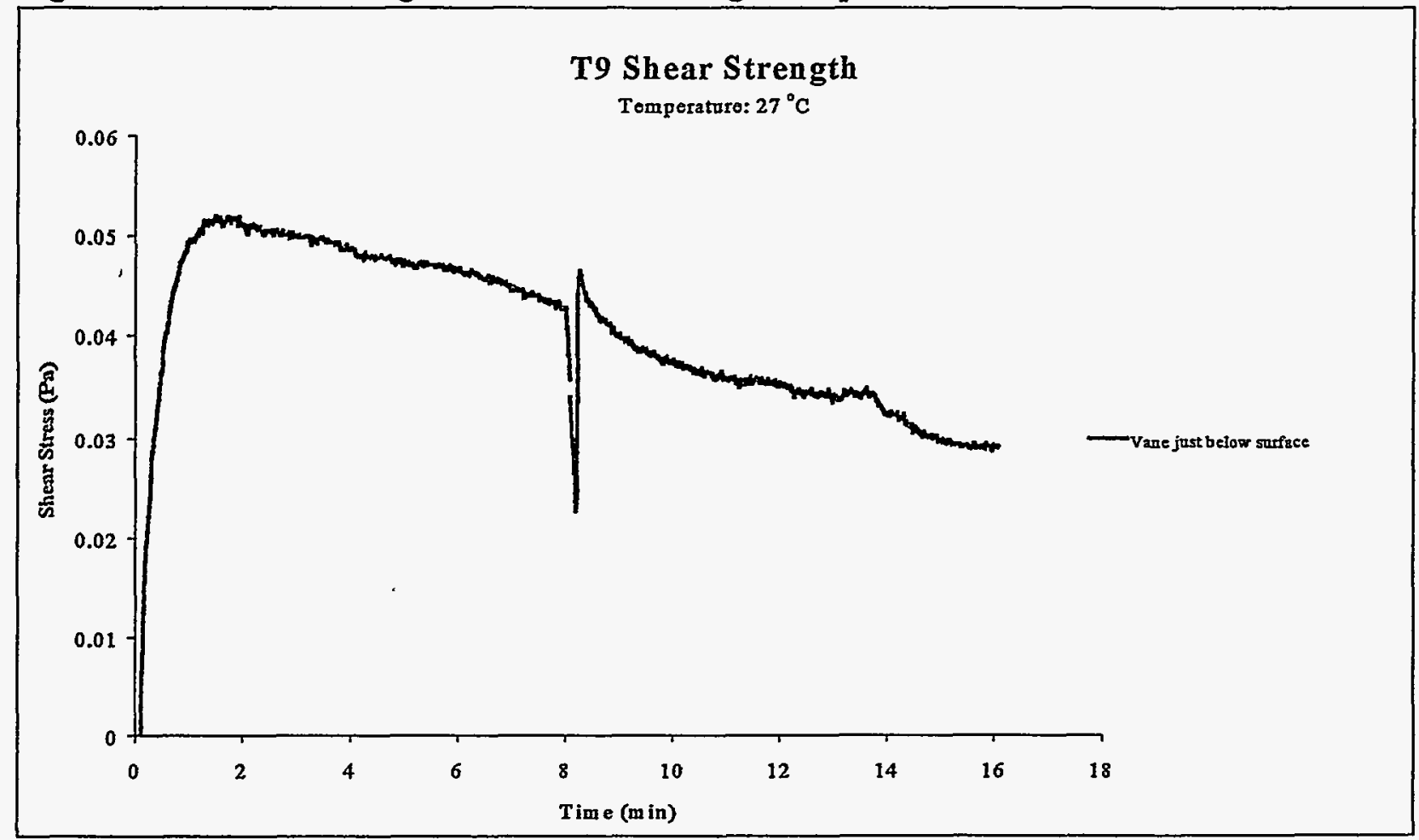




\subsection{Viscosity and Flow Curves}

The same sludge composites prepared for the shear strength tests were also employed for the viscosity experiments. Each composite sludge was mixed and diluted with the respective supernatant to an approximate ratio of 1:1. From this diluted sludge mixture, an aliquot was removed for a total solids measurement. The total solids content for each diluted sludge sample employed for the viscosity measurements is documented on the respective viscosity curves The apparent viscosity and flow characteristics were measured over a range of increasing $\left(0.0 \mathrm{~s}^{-1}\right.$ to 450 $\left.\mathrm{s}^{-1}\right)$ to decreasing $\left(450 \mathrm{~s}^{-1}\right.$ to $\left.0.0 \mathrm{~s}^{-1}\right)$ shear rates. A time interval of three minutes was selected for both the increasing and decreasing shear rate steps. The "up curve" represents the increasing shear rate and is annotated in blue and the corresponding "down curve" for decreasing shear rate is shown in red. The temperature throughout the measurements was maintained at $25^{\circ} \mathrm{C} \pm 0.5^{\circ} \mathrm{C}$. The tests were performed using the Haake immersion sensor system. The system is comprised of a hollow immersion tube with an inner diameter of $42 \mathrm{~mm}$ and a modified cylindrical MV DIN rotor with a diameter of $36.8 \mathrm{~mm}$ that is placed within the tube. This sensor system was modified to increase the annular distance from $3.3 \mathrm{~mm}$ to $5.2 \mathrm{~mm}$ between the rotor and tube wall, which is the region where the sample flows during the test. This annular distance was enlarged to help minimize the effect of large particles on the shear stress data collected during the viscosity and flow measurements.

Prior to the start of all tests, the samples were stabilized at a temperature of $25^{\circ} \mathrm{C} \pm 0.5^{\circ} \mathrm{C}$ with a circulating water bath. During the temperature stabilization process each sample was stirred to suspend the slurry for the test. Typically this process took approximately $10 \mathrm{~min}$. to complete. Once the system equilibrated at the desired temperature the sensor was lowered into the sample and the stirring of the sample was suspended. At this point rotation of the inner cylinder was initiated along with the data collection for the flow measurements. The viscosity and flow curves for each OHF tank sludge are illustrated in Figures 15-24. An expanded view of the viscosity curves is provided for each tank sample to better illustrate the change in viscosity over the range of shear rates used in the tests.

The initial jump in shear stress, observed in each flow curve, is due to the torque necessary to start the MV DIN rotating and the magnitude of this initial jump on the increasing shear rate or "up 
curve" provides an estimate of the shear strength $\left(\tau_{s}\right)$. The magnitude of the final drop in shear stress to zero on the "down curve" is considered by many users of rheometry data to an estimate of the yield stress $\left(\tau_{y}\right)$ for the fluid. The break in the flow curves that generally occurs above a shear rate of about $150 \mathrm{~s}^{-1}$ for the OHF sludge samples is the transition from laminar flow to turbulent flow within the annular region of the cylindrical MV DIN rotor. Therefore, any measurements above this transition point or the use of shear rates above the region of laminar flow provides no useful flow data. An estimate of the maximum shear rates that can be used before experiencing turbulent flow behavior are summarized in Table 35 for the OHF sludge samples along with estimates for the shear strength $\left(\tau_{s}\right)$ and yield stress $\left(\tau_{y}\right)$ for each sludge.

\section{Table 35 Estimation of flow properties for the OHF sludge samples}

\begin{tabular}{||c|c|c|c||}
\hline \hline $\begin{array}{c}\text { Sludge } \\
\text { sample }\end{array}$ & $\begin{array}{c}\text { Shear } \\
\text { strength } \\
(\mathbf{P a})\end{array}$ & $\begin{array}{c}\text { Yield } \\
\text { stress } \\
(\mathbf{P a})\end{array}$ & $\begin{array}{c}\text { Maximum } \\
\text { Shear rate } \\
\left(\mathbf{s}^{-1}\right)\end{array}$ \\
\hline \hline $\mathrm{T} 1$ & 2.4 & 1.9 & 240 \\
\hline $\mathrm{T} 2$ & 0.9 & 0.4 & 180 \\
\hline $\mathrm{T} 3$ & 1.2 & 0.4 & 140 \\
\hline $\mathrm{T} 4$ & 4.4 & 3.9 & 300 \\
\hline $\mathrm{T} 9$ & 2.2 & 2.0 & 240 \\
\hline
\end{tabular}

There was some concern about the shear strength estimates by the MV DIN rotor for a diluted (1:1) sludge sample being so much greater than the shear strength measured using the shear vane rotor on an undiluted sludge. All rotor constants were checked for accuracy and were determined to be correct. The vane rotor and the concentric cylinder rotor have radically different geometries with a large difference in the surface area that comes into contact with the sample. If a comparison were desired a conversion factor would need to be determined for the two rotors to normalize the data. It is not uncommon to see shear stress measurements that differ by orders of magnitude between rotors of different design. There is currently insufficient history with the ORNL sludge samples to derive a normalization factor that would allow comparison of the different rotor designs. 
Figure 15 Viscosity vs. Shear Rate for T1 Sludge Composite

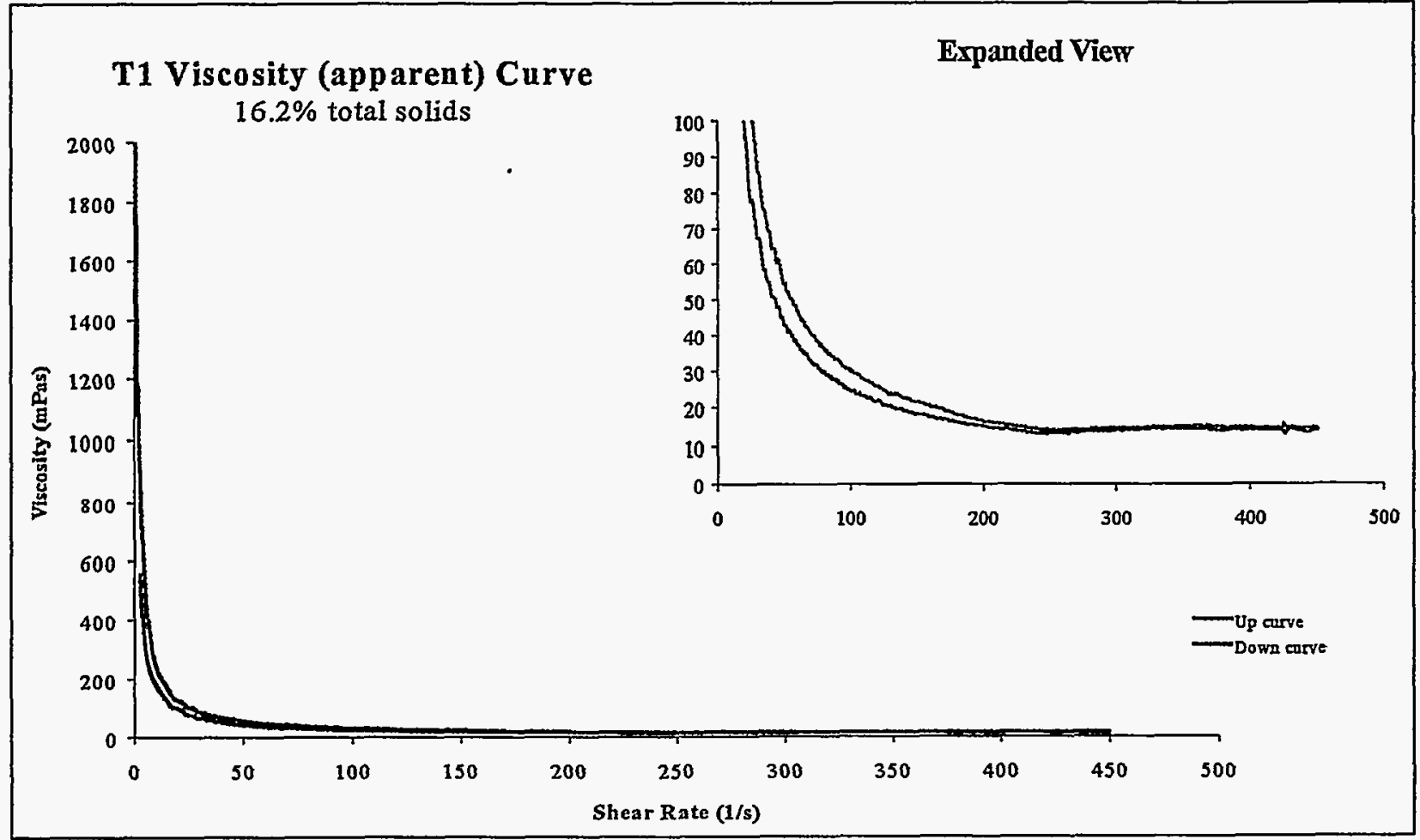

Figure 16 Shear Stress vs. Shear Rate for T1 Sludge Composite

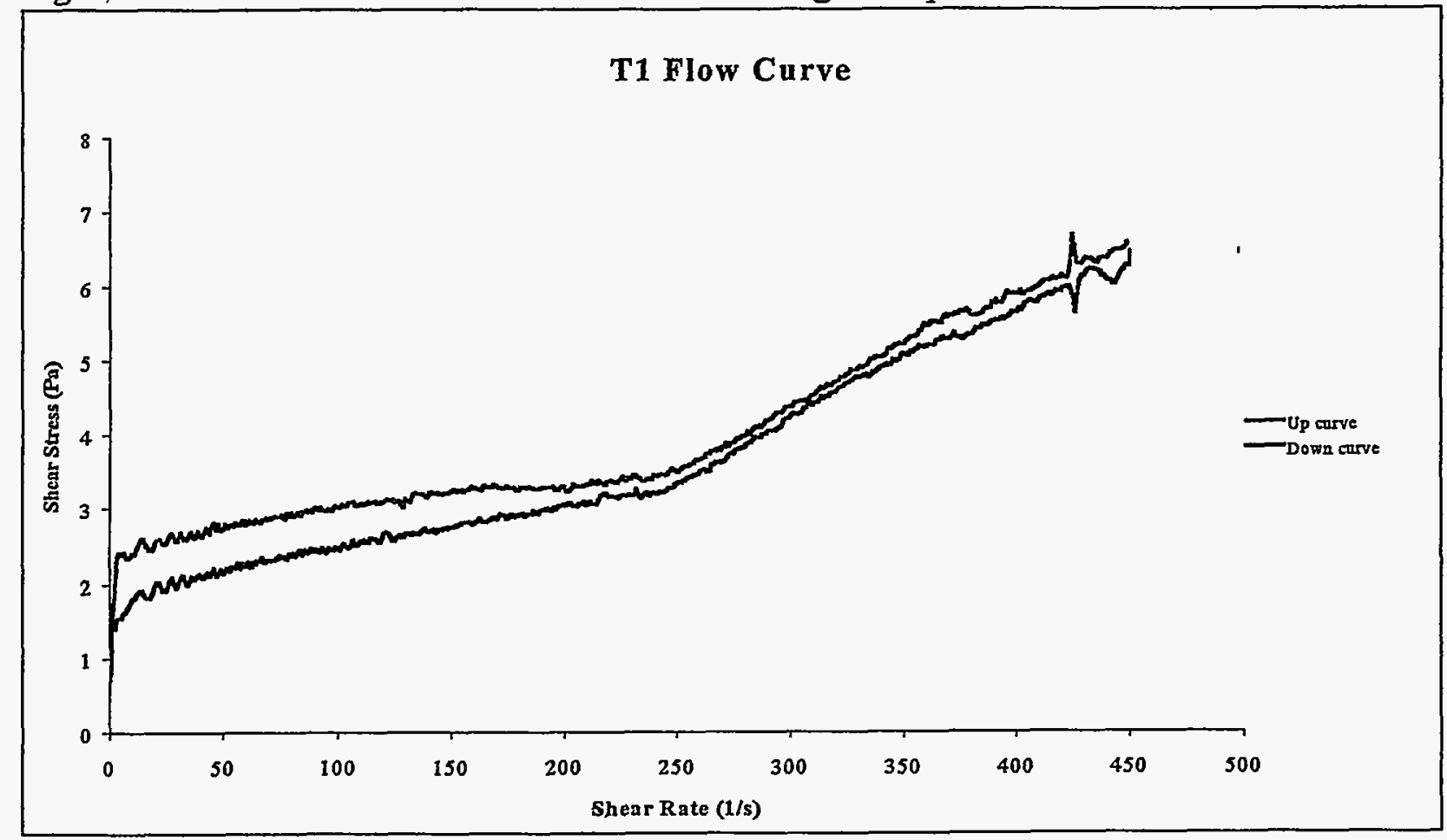


Figure 17 Viscosity vs, Shear Rate for T2 Sludge Composite

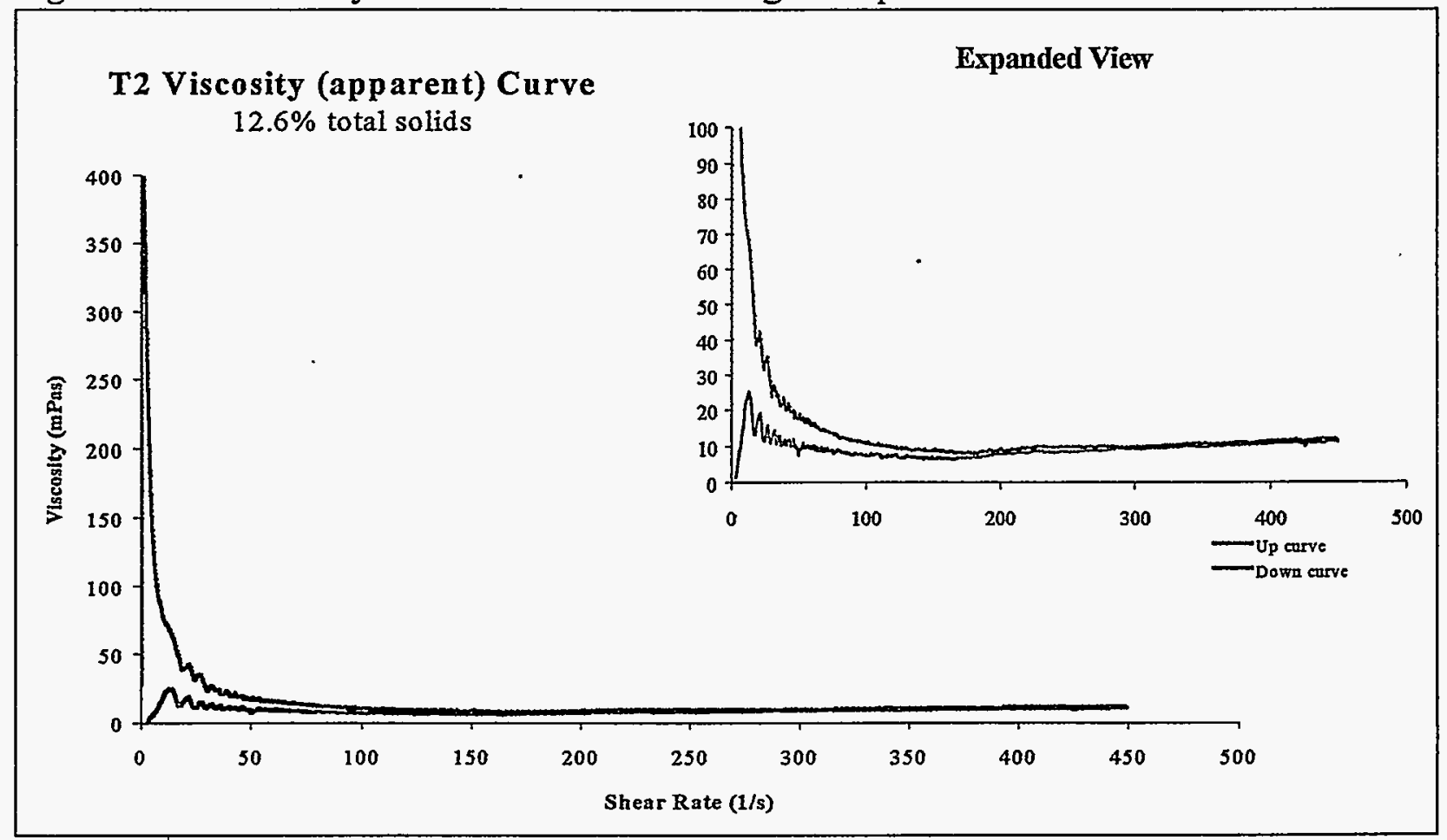

Figure 18 Shear Stress vs. Shear Rate for T2 Sludge Composite

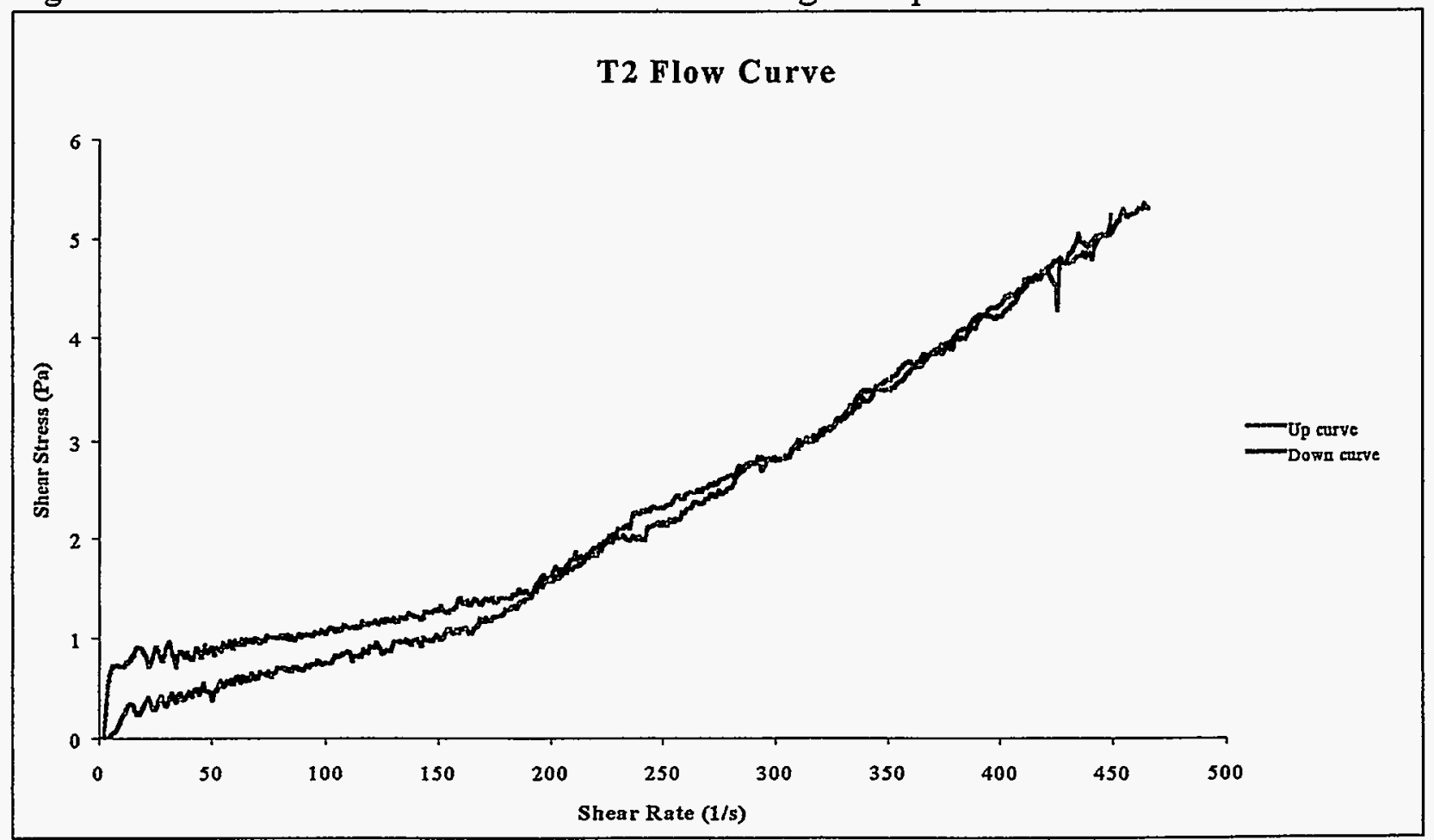


Figure 19 Viscosity vs. Shear Rate for T3 Sludge Composite

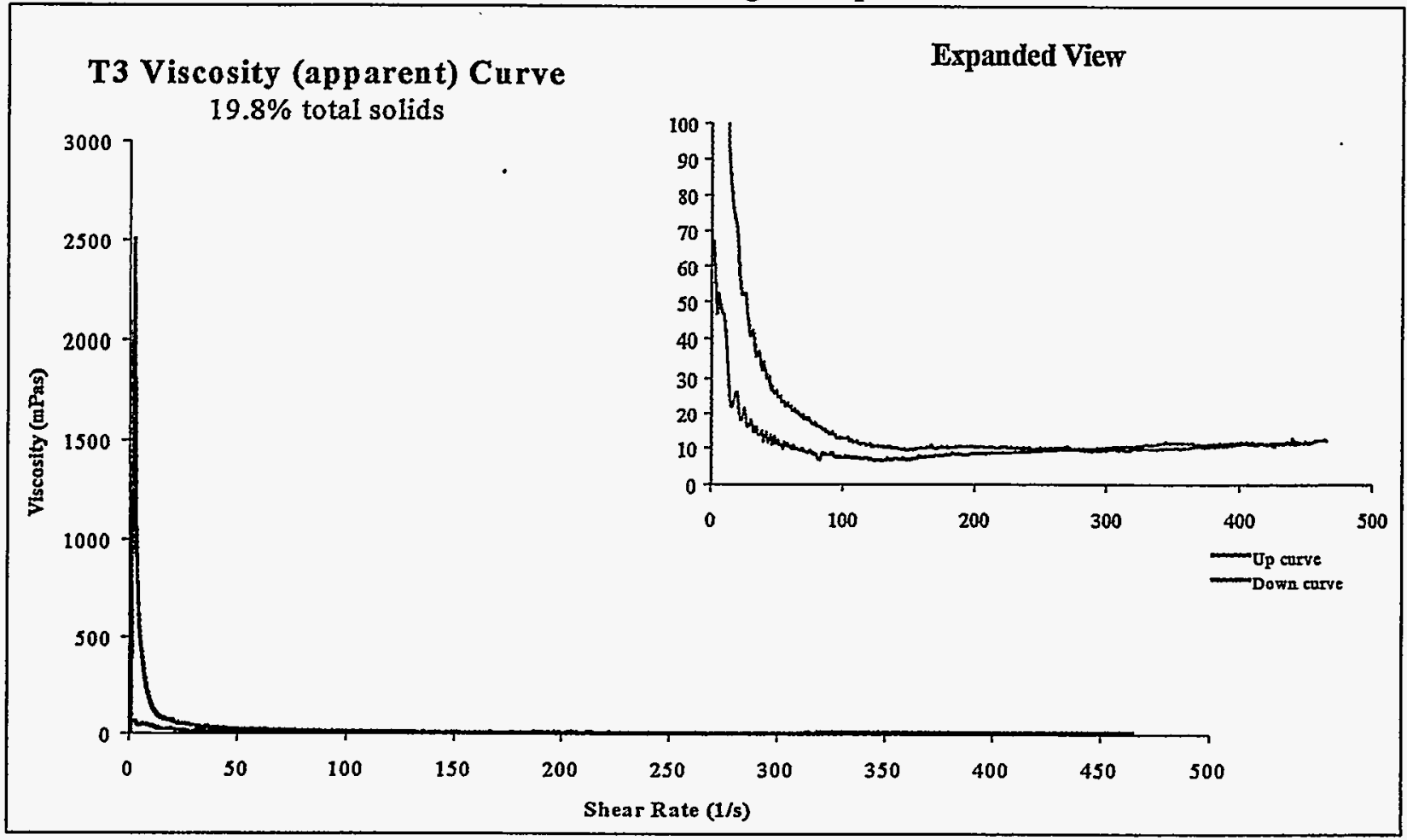

Figure 20 Shear Stress vs. Shear Rate for T3 Sludge Composite

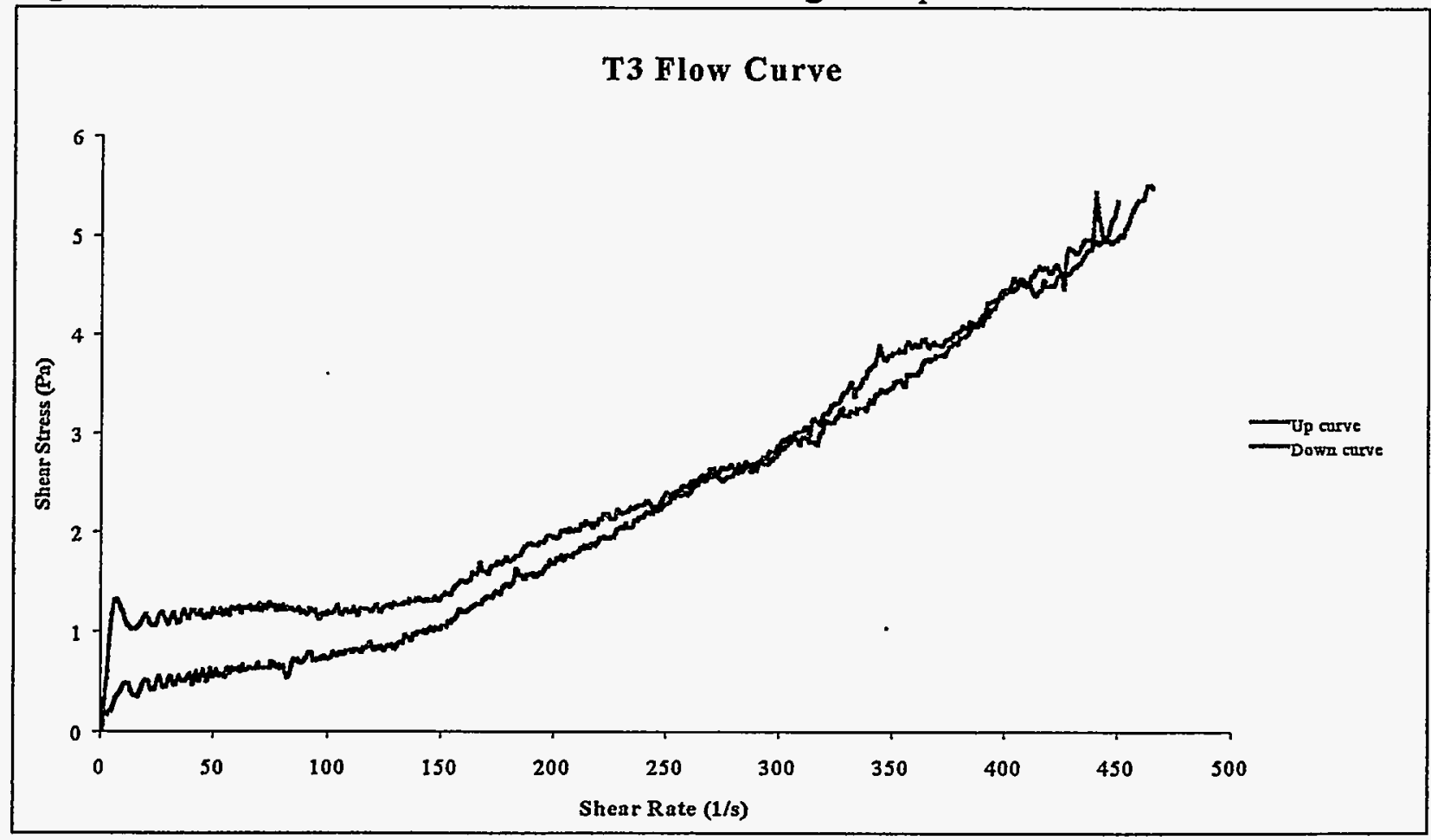


Figure 21 Viscosity vs. Shear Rate for T4 Sludge Composite

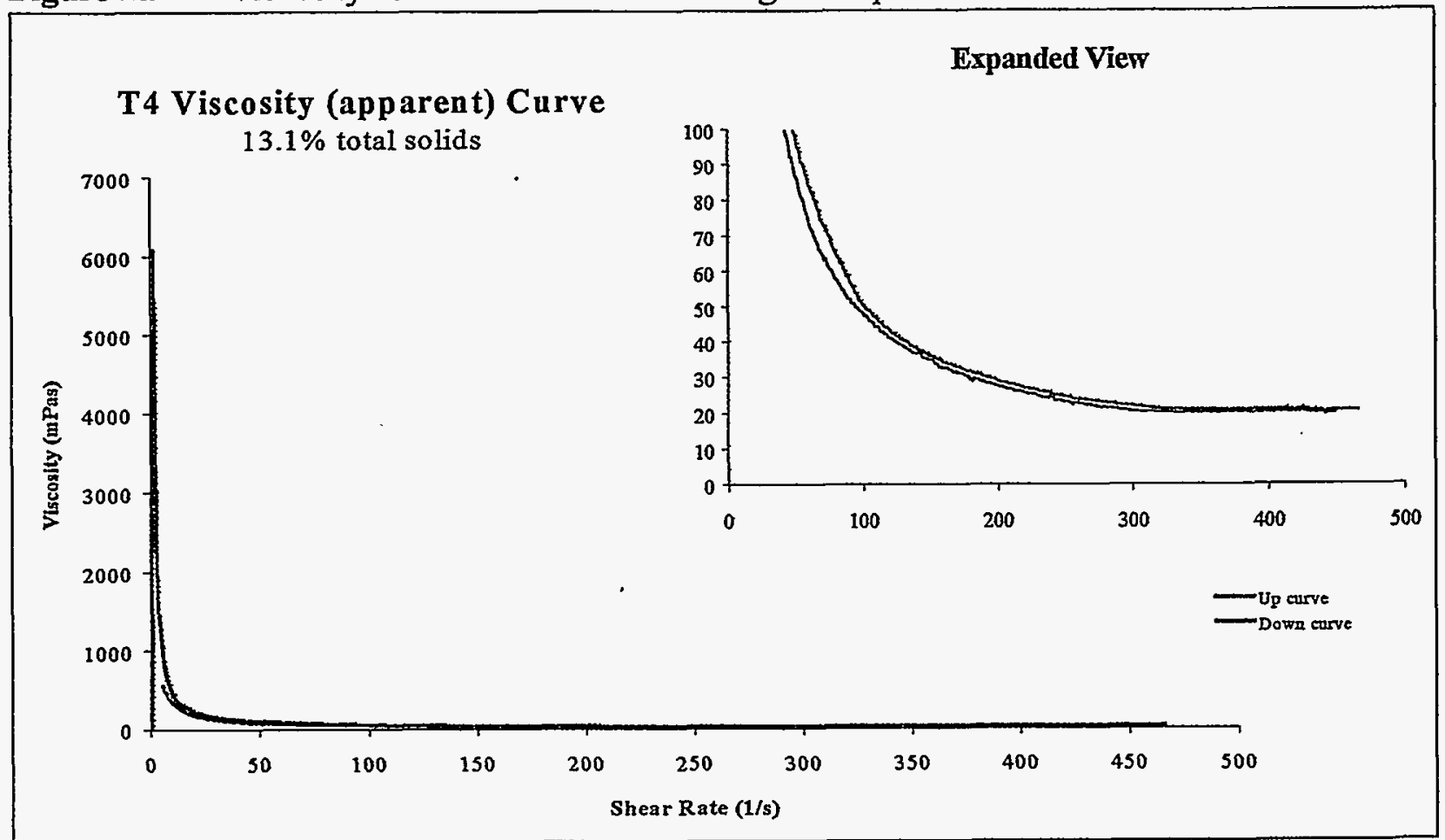

Figure 22 Shear Stress vs. Shear Rate for T4 Sludge Composite

\section{T4 Flow Curve}

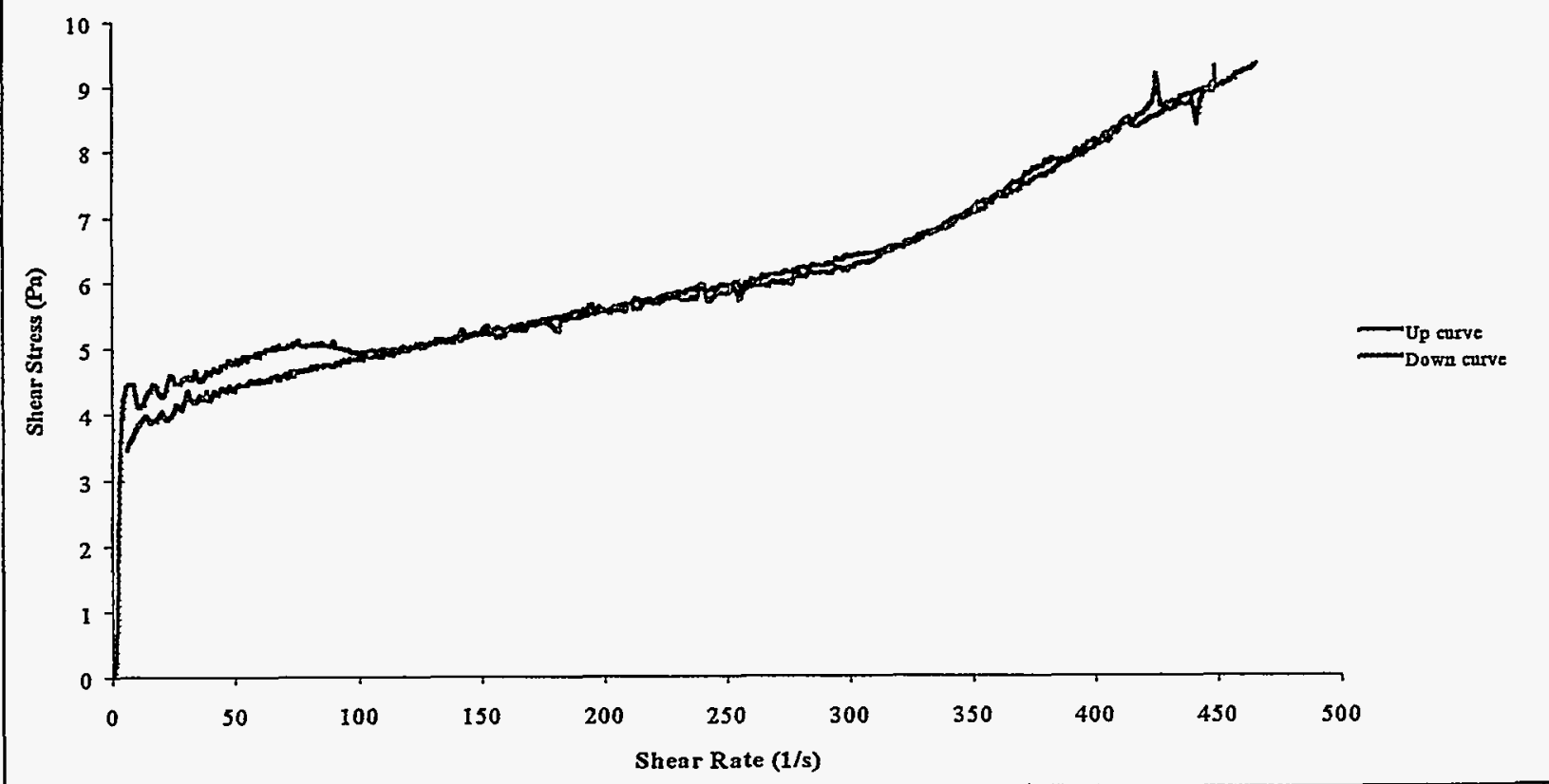


Figure 23 Viscosity vs. Shear Rate for T9 Sludge Composite

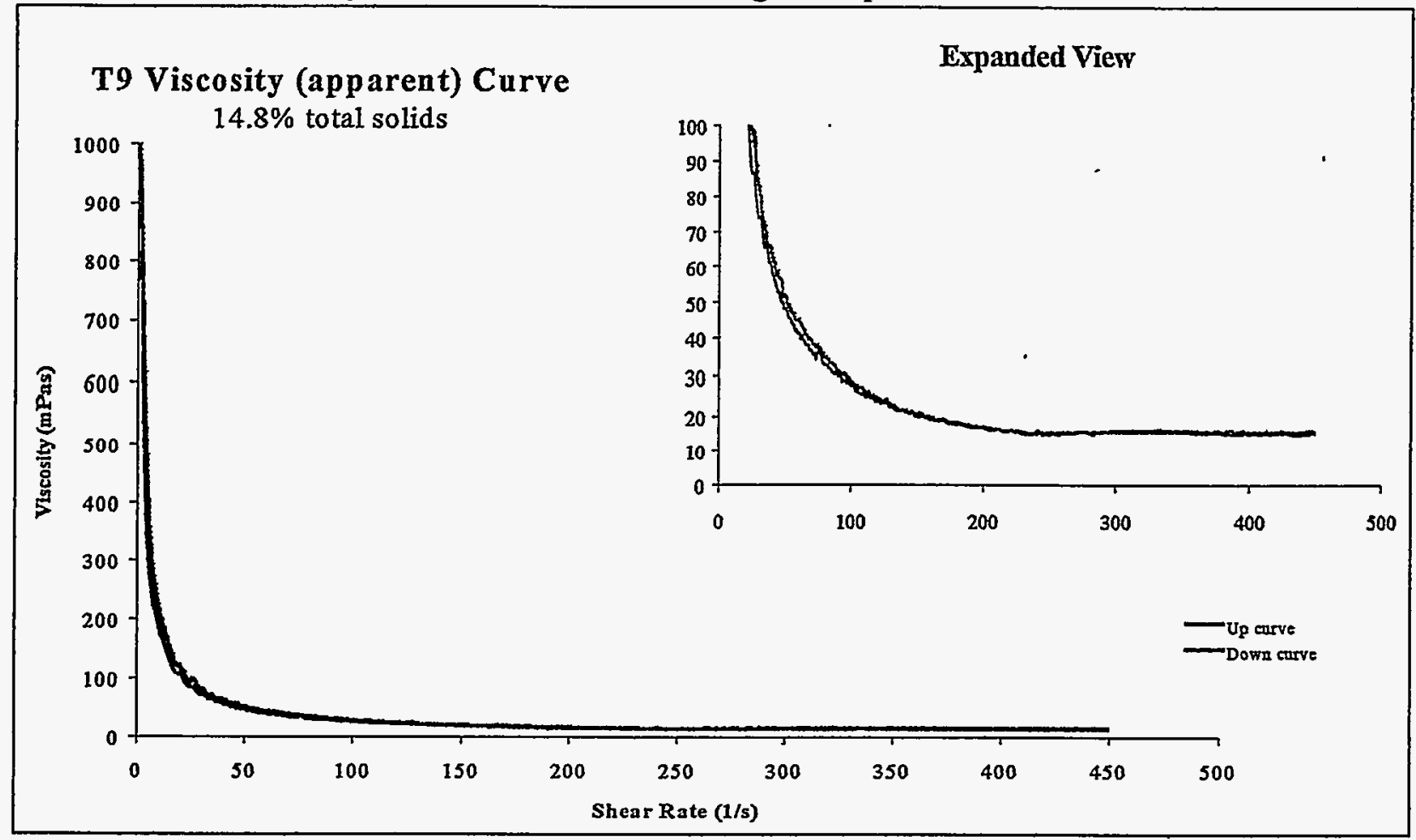

Figure 24 Shear Stress vs. Shear Rate for T9 Sludge Composite

T9 Flow Curve

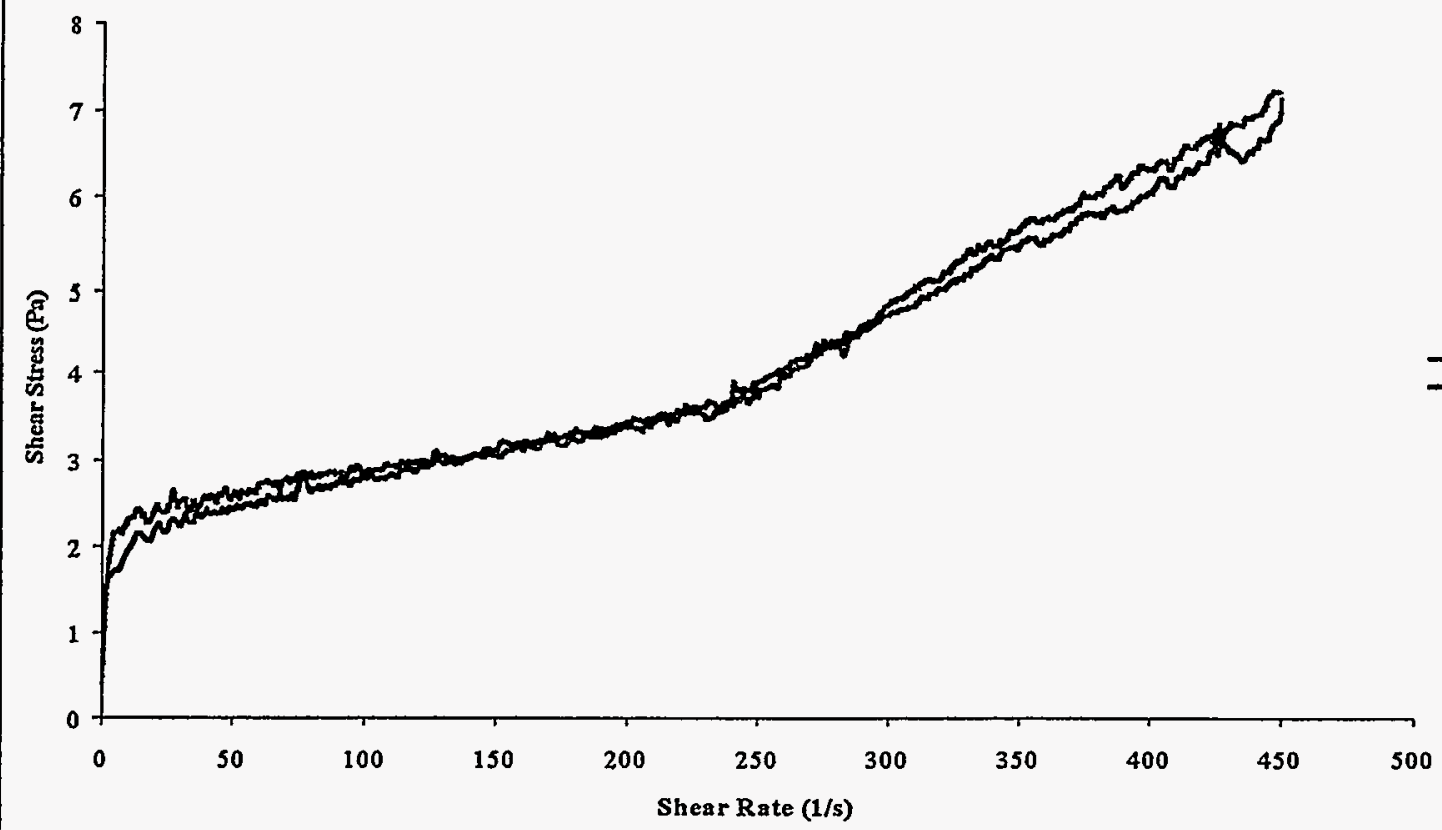


122 


\subsection{Statistical Description of OHF Tank Data}

This report summarizes the waste characterization data for the five OHF tanks (T1-T4, and T9) and includes both liquid and sludge samples collected from three lateral locations across each waste tank. Since both the chemical and radionuclide content is mostly concentrated in the sludge present in each tank the discussions concerning the data heterogeneity are directed toward the sludge composition. Once all the ORNL sludge waste is transferred to the MVST system the waste from the OHF tanks will represent only about $3 \%$ (see Table 36 ) of the total sludge mass and volume.

Table 36 Contribution of OHF sludge to overall ORNL sludge inventory

\begin{tabular}{|c|c|c|}
\hline Tank & $\begin{array}{c}\text { Percent }^{*} \\
\text { of } \\
\text { Total Sludge Mass } \\
(\mathbf{1 , 0 1 1 , 1 4 3 ~ K g )}\end{array}$ & $\begin{array}{c}\text { Percent }^{*} \\
\text { of } \\
\text { Total Sludge Volume } \\
(\mathbf{1 9 9 , 7 0 0} \text { gal. })\end{array}$ \\
\hline \hline $\mathrm{T} 1$ & 0.398 & 0.401 \\
\hline $\mathrm{T} 2$ & 0.647 & 0.651 \\
\hline $\mathrm{T} 3$ & 1.030 & 1.052 \\
\hline $\mathrm{T} 4$ & 0.639 & 0.701 \\
\hline $\mathrm{T} 9$ & 0.217 & 0.250 \\
\hline Total & $\mathbf{2 . 9 3 1}$ & $\mathbf{3 . 0 5 5}$ \\
\hline \hline
\end{tabular}

- C. K. Bayne, et. al., Statistical Description of Liquid Low-Level Waste System Transuranic Wastes at Oak Ridge National Laboratory, Oak Ridge, Tennessee, ORNL/TM-13352 (December 1996), see Table 3.7. 
The simple statistics that are calculated and summarized in the following tables and graphs include the mean (average), standard deviation, and the relative error. These statistics are defined as follows:

$$
\begin{gathered}
\text { mean }=\bar{x}=\frac{1}{n} \sum_{j=1}^{n} x_{j} \\
\text { standard deviation }=\sigma=\sqrt{\frac{1}{(n-1)} \sum_{j=1}^{n}\left(x_{j}-\bar{x}\right)^{2}} \\
2 \sigma \cong 95 \% \text { confidence interval } \\
\text { Percent Relative Error }=\left(\frac{2 \sigma}{\bar{x}}\right) \cdot 100 \%
\end{gathered}
$$

\subsection{Statistical summary for the major metals}

A summary of the concentrations for the major metals observed at each sample location are tabulated for each tank in Tables 37-41, along with the average, $2 \sigma$ error, and the $2 \sigma$ percent relative error. The same information for the selected metals across all five tanks collectively is provide in Table 42. The variations in the metal data for each tank are illustrated by four different perspectives in Fig. 25 through Fig. 44. 
Table 37 Summary statistics for major metals in T1 sludge

\begin{tabular}{|crrrrrr||}
\hline \multicolumn{7}{|c|}{$\begin{array}{c}\text { T1 } \\
\text { (mg/Kg) }\end{array}$} \\
\cline { 2 - 7 } Element & North & Center & South & average & 20 & $\% 20$ \\
\hline \hline Th & 43000 & 90500 & 39900 & 57800 & 56723 & $98.1 \%$ \\
$\mathrm{Ca}$ & 12600 & 27900 & 21700 & 20733 & 15391 & $74.2 \%$ \\
$\mathrm{Al}$ & 4660 & 26200 & 19900 & 16920 & 22150 & $130.9 \%$ \\
$\mathrm{Fe}$ & 15000 & 3440 & 10500 & 9647 & 11654 & $120.8 \%$ \\
$\mathrm{Na}$ & 3860 & 4040 & 3180 & 3693 & 907 & $24.6 \%$ \\
$\mathrm{Mg}$ & 1400 & 3460 & 2000 & 2287 & 2119 & $92.7 \%$ \\
$\mathrm{U}$ & 1410 & 2420 & 1360 & 1730 & 1196 & $69.1 \%$ \\
$\mathrm{~K}$ & 2620 & 1680 & 2090 & 2130 & 943 & $44.3 \%$ \\
$\mathrm{Sr}$ & 231 & 946 & 727 & 635 & 733 & $115.4 \%$ \\
$\mathrm{Mn}$ & 287 & 318 & 369 & 325 & 83 & $25.5 \%$ \\
\hline
\end{tabular}

Table 38 Summary statistics for major metals in T2 sludge

\begin{tabular}{|crrrrrr||}
\hline \multicolumn{7}{|c|}{$\begin{array}{c}\text { T2 } \\
\text { (mg/Kg) }\end{array}$} \\
\cline { 2 - 7 } Element & North & Center & South & average & \multicolumn{1}{c|}{20} & $\% 20$ \\
\hline \hline $\mathrm{Th}$ & 24200 & 94300 & 54800 & 57767 & 70288 & $121.7 \%$ \\
$\mathrm{Ca}$ & 12900 & 36600 & 12900 & 20800 & 27366 & $131.6 \%$ \\
$\mathrm{Al}$ & 9390 & 15900 & 20300 & 15197 & 10978 & $72.2 \%$ \\
$\mathrm{Fe}$ & 48900 & 62400 & 2940 & 38080 & 62343 & $163.7 \%$ \\
$\mathrm{Na}$ & 3870 & 5060 & 4500 & 4477 & 1191 & $26.6 \%$ \\
$\mathrm{Mg}$ & 2580 & 3170 & 1410 & 2387 & 1792 & $75.1 \%$ \\
$\mathrm{U}$ & 832 & 2090 & 1350 & 1424 & 1265 & $88.8 \%$ \\
$\mathrm{~K}$ & 2270 & 2130 & 2490 & 2297 & 363 & $15.8 \%$ \\
$\mathrm{Sr}$ & 261 & 992 & 460 & 571 & 756 & $132.4 \%$ \\
$\mathrm{Mn}$ & 555 & 336 & 264 & 385 & 303 & $78.7 \%$ \\
\hline
\end{tabular}

Table 39 Summary statistics for major metals in T3 sludge

\begin{tabular}{|crrrrrr||}
\hline \multicolumn{7}{|c|}{$\begin{array}{c}\text { T3 } \\
\text { (mq/Kg) }\end{array}$} \\
\cline { 2 - 7 } Element & North & Center & South & average & $2 \sigma$ & $\% 2 \sigma$ \\
\hline \hline $\mathrm{Th}$ & 36100 & 77500 & 44600 & 52733 & 43731 & $82.9 \%$ \\
$\mathrm{Ca}$ & 27000 & 37900 & 24300 & 29733 & 14400 & $48.4 \%$ \\
$\mathrm{Al}$ & 15600 & 11100 & 11733 & 7184 & $61.2 \%$ \\
$\mathrm{Fe}$ & 7500 & 4790 & 4420 & 5700 & 3650 & $64.0 \%$ \\
$\mathrm{Na}$ & 4890 & 18800 & 15600 & 16667 & 3695 & $22.2 \%$ \\
$\mathrm{Mg}$ & 15600 & 3570 & 2780 & 3077 & 860 & $28.0 \%$ \\
$\mathrm{U}$ & 2880 & 3910 & 4333 & 2846 & $65.7 \%$ \\
$\mathrm{~K}$ & 3170 & 5920 & 5200 & 5643 & 945 & $16.7 \%$ \\
$\mathrm{Sr}$ & 5590 & 6140 & 204 & 223 & 105 & $47.2 \%$ \\
$\mathrm{Mn}$ & 182 & 282 & 164 & 167 & 62 & $37.3 \%$ \\
\hline
\end{tabular}


Table 40 Summary statistics for major metals in T4 sludge

\begin{tabular}{|c|c|c|c|c|c|c|}
\hline \multirow[b]{2}{*}{ Element } & \multicolumn{6}{|c|}{$\begin{array}{c}\mathrm{T4} \\
(\mathrm{mg} / \mathrm{Kg})\end{array}$} \\
\hline & North & Center & South & average & 20 & $\% 2 \sigma$ \\
\hline Th & 39100 & 124000 & 45200 & 69433 & 94709 & $136.4 \%$ \\
\hline $\mathrm{Ca}$ & 9950 & 20600 & 10400 & 13650 & 12046 & $88.3 \%$ \\
\hline $\mathrm{Al}$ & 17900 & 9320 & 17700 & 14973 & 9794 & $65.4 \%$ \\
\hline $\mathrm{Fe}$ & 3540 & 3150 & 2870 & 3187 & 673 & $21.1 \%$ \\
\hline $\mathrm{Na}$ & 5330 & 7400 & 5870 & 6200 & 2147 & $34.6 \%$ \\
\hline $\mathrm{Mg}$ & 1530 & 1730 & 1230 & 1497 & 503 & $33.6 \%$ \\
\hline $\mathrm{U}$ & 3130 & 7870 & 3270 & 4757 & 5394 & $113.4 \%$ \\
\hline $\mathrm{K}$ & 2240 & 2080 & 2350 & 2223 & 272 & $12.2 \%$ \\
\hline $\mathrm{Sr}$ & 175 & 334 & 204 & 238 & 169 & $71.3 \%$ \\
\hline $\mathrm{Mn}$ & 360 & 472 & 277 & 370 & 196 & $52.9 \%$ \\
\hline
\end{tabular}

Table 41 Summary statistics for major metals in T9 sludge

\begin{tabular}{|crrrrrr||}
\hline \multicolumn{7}{|c|}{$\begin{array}{c}\text { T9 } \\
\text { (mg/Kg) }\end{array}$} \\
\cline { 2 - 7 } Element & North & Center & South & average & \multicolumn{1}{c|}{$2 \sigma$} & $\%$ 20 \\
\hline \hline Th & 76800 & 56800 & 54800 & 62800 & 24331 & $38.7 \%$ \\
$\mathrm{Ca}$ & 28900 & 32800 & 41100 & 34267 & 12462 & $36.4 \%$ \\
$\mathrm{Al}$ & 4110 & 34500 & 14900 & 17837 & 30813 & $172.7 \%$ \\
$\mathrm{Fe}$ & 21900 & 17900 & 7740 & 15847 & 14600 & $92.1 \%$ \\
\hline $\mathrm{Na}$ & 5940 & 6640 & 5280 & 5953 & 1360 & $22.8 \%$ \\
$\mathrm{Mg}$ & 1520 & 5140 & 2190 & 2950 & 3852 & $130.6 \%$ \\
$\mathrm{U}$ & 2700 & 2510 & 2220 & 2477 & 483 & $19.5 \%$ \\
$\mathrm{~K}$ & 826 & 974 & 769 & 856 & 212 & $24.7 \%$ \\
$\mathrm{Sr}$ & 751 & 908 & 2400 & 1353 & 1820 & $134.5 \%$ \\
$\mathrm{Mn}$ & 327 & 337 & 252 & 305 & 93 & $30.4 \%$ \\
\hline \hline
\end{tabular}

Table 42 Summary statistics for major metals in T1-T9 sludge

\begin{tabular}{|c|c|c|c|c|c|c|}
\hline \multirow[b]{2}{*}{ Element } & \multicolumn{5}{|c|}{$\begin{array}{c}\text { T1-T9 } \\
\text { (mg/Kg) }\end{array}$} & \multirow[b]{2}{*}{$\% 20$} \\
\hline & North & Center & South & average & $2 \sigma$ & \\
\hline Th & 43840 & 88620 & 47860 & 60107 & 49550 & $82.4 \%$ \\
\hline $\mathrm{Ca}$ & 18270 & 31160 & 22080 & 23837 & 13244 & $55.6 \%$ \\
\hline $\mathrm{Al}$ & 8912 & 20304 & 16780 & 15332 & 11665 & $76.1 \%$ \\
\hline $\mathrm{Fe}$ & 18846 & 18936 & 5694 & 14492 & 15239 & $105.2 \%$ \\
\hline $\mathrm{Na}$ & 6920 & 8388 & 6886 & 7398 & 1715 & $23.2 \%$ \\
\hline $\mathrm{Mg}$ & 1982 & 3414 & 1922 & 2439 & 1689 & $69.2 \%$ \\
\hline $\mathrm{U}$ & 2248 & 4162 & 2422 & 2944 & 2117 & $71.9 \%$ \\
\hline $\mathrm{K}$ & 2709 & 2601 & 2580 & 2630 & 139 & $5.3 \%$ \\
\hline Sr & 320 & 692 & 799 & 604 & 503 & $83.3 \%$ \\
\hline $\mathrm{Mn}$ & 333 & 332 & 265 & 310 & 78 & $25.2 \%$ \\
\hline
\end{tabular}


Figure 25 Lateral Distribution of Major Metals in T1 Sludge

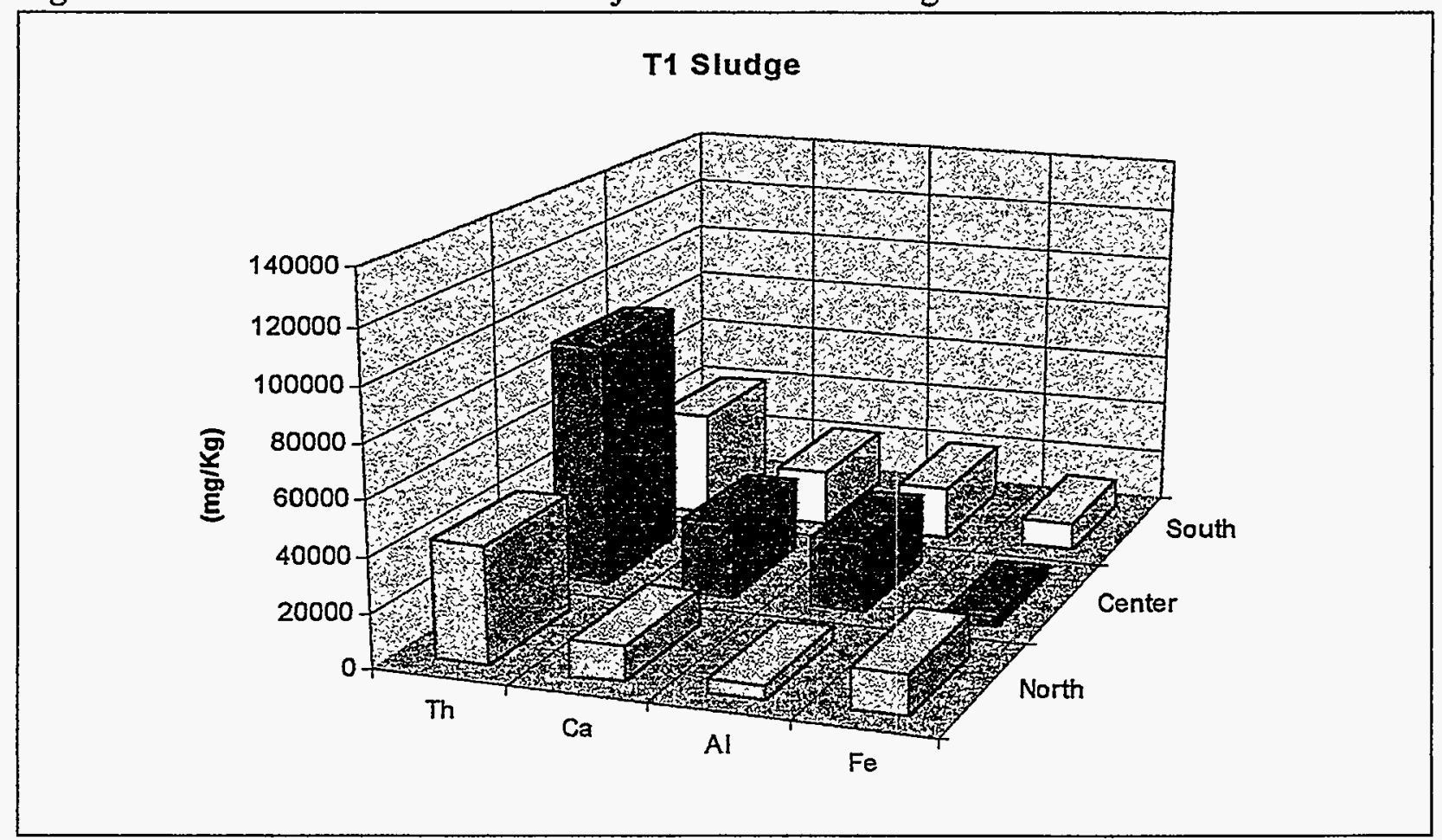

Figure 26 Lateral Distribution of Selected Metals in T1 Sludge

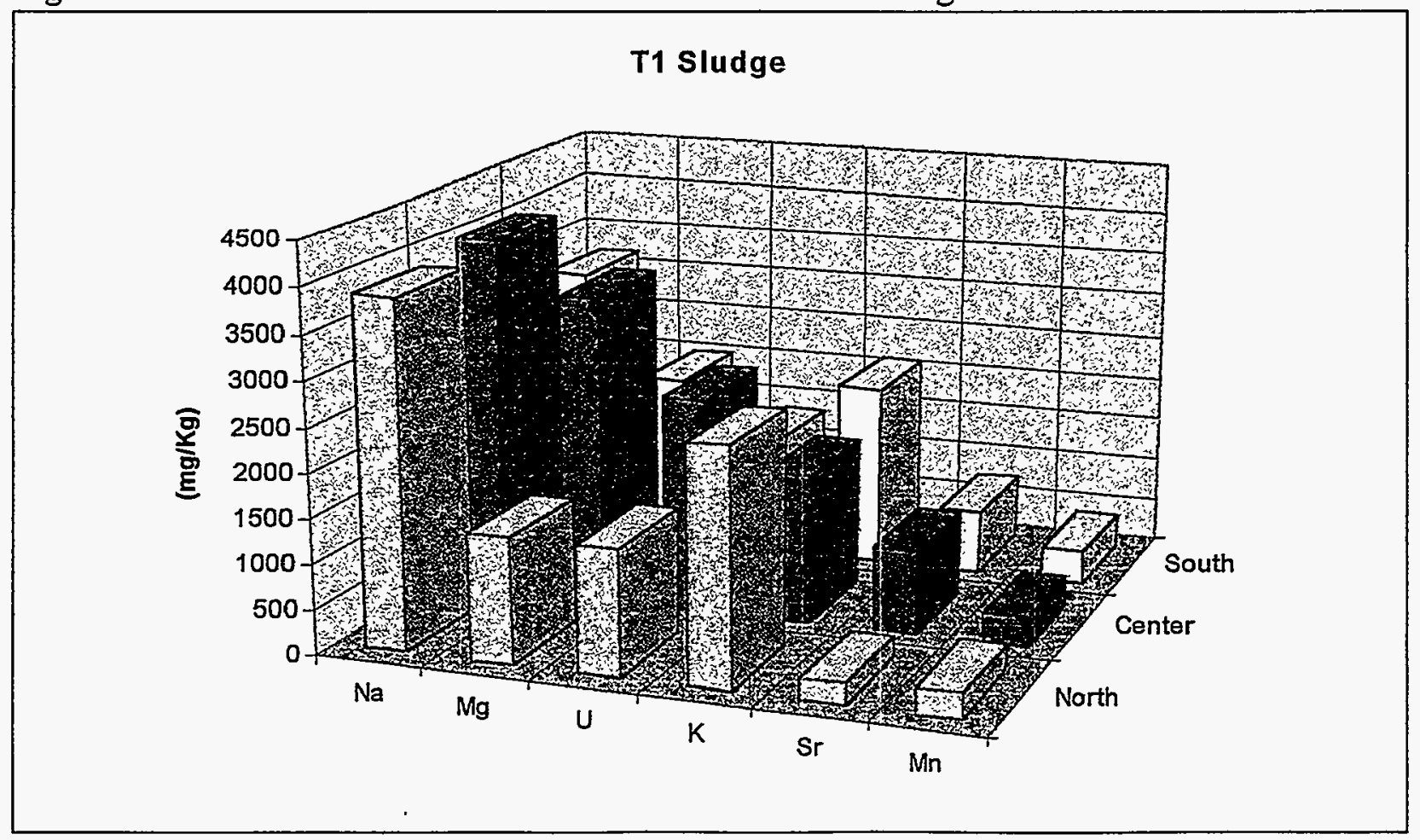


Figure 27 Statistical Distribution of Major Metals from Lateral Sampling of T1 Sludge T1 Sludge

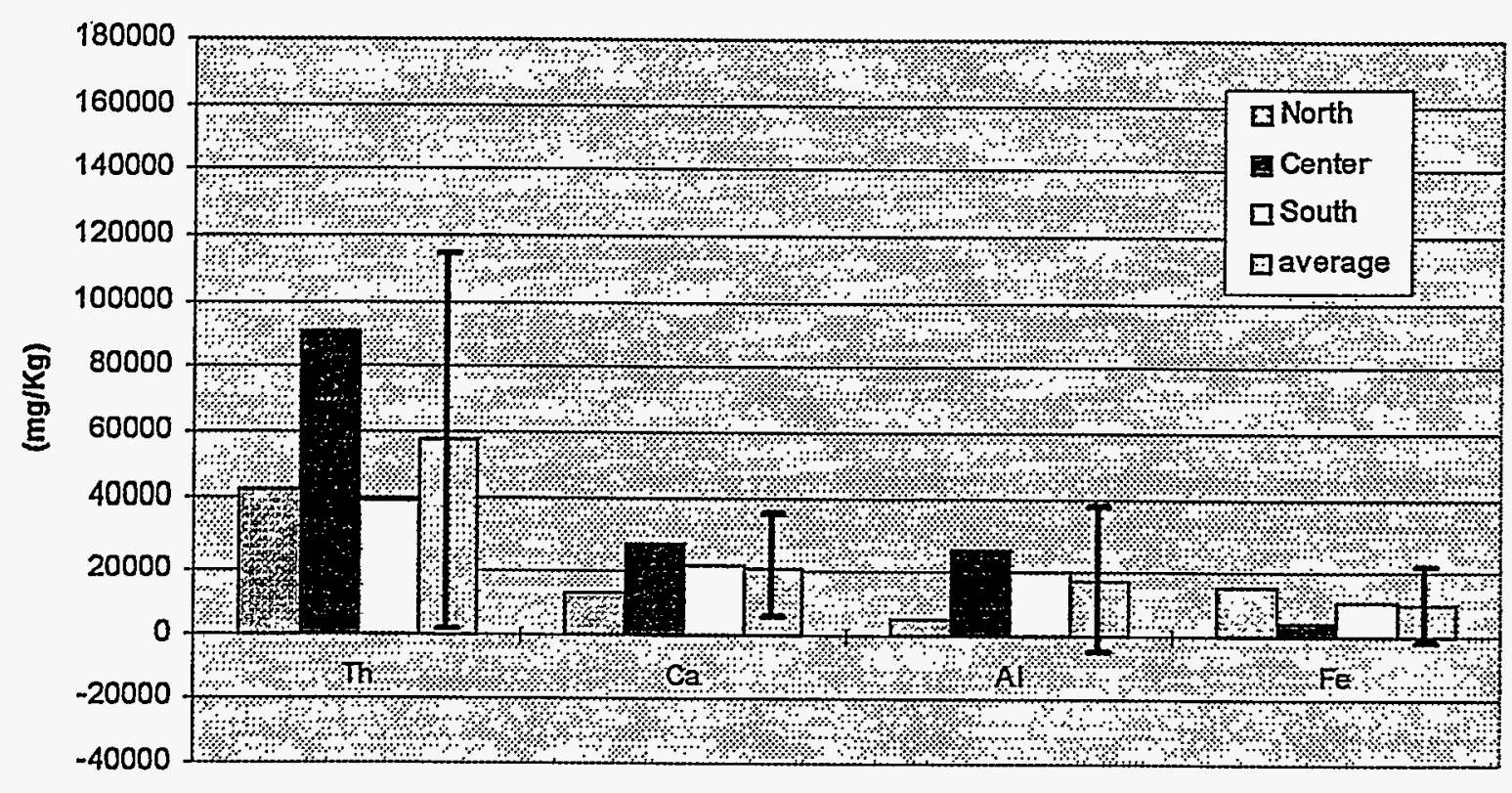

Figure 28 Statistical Distribution of Selected Metals from Lateral Sampling of T1 Sludge

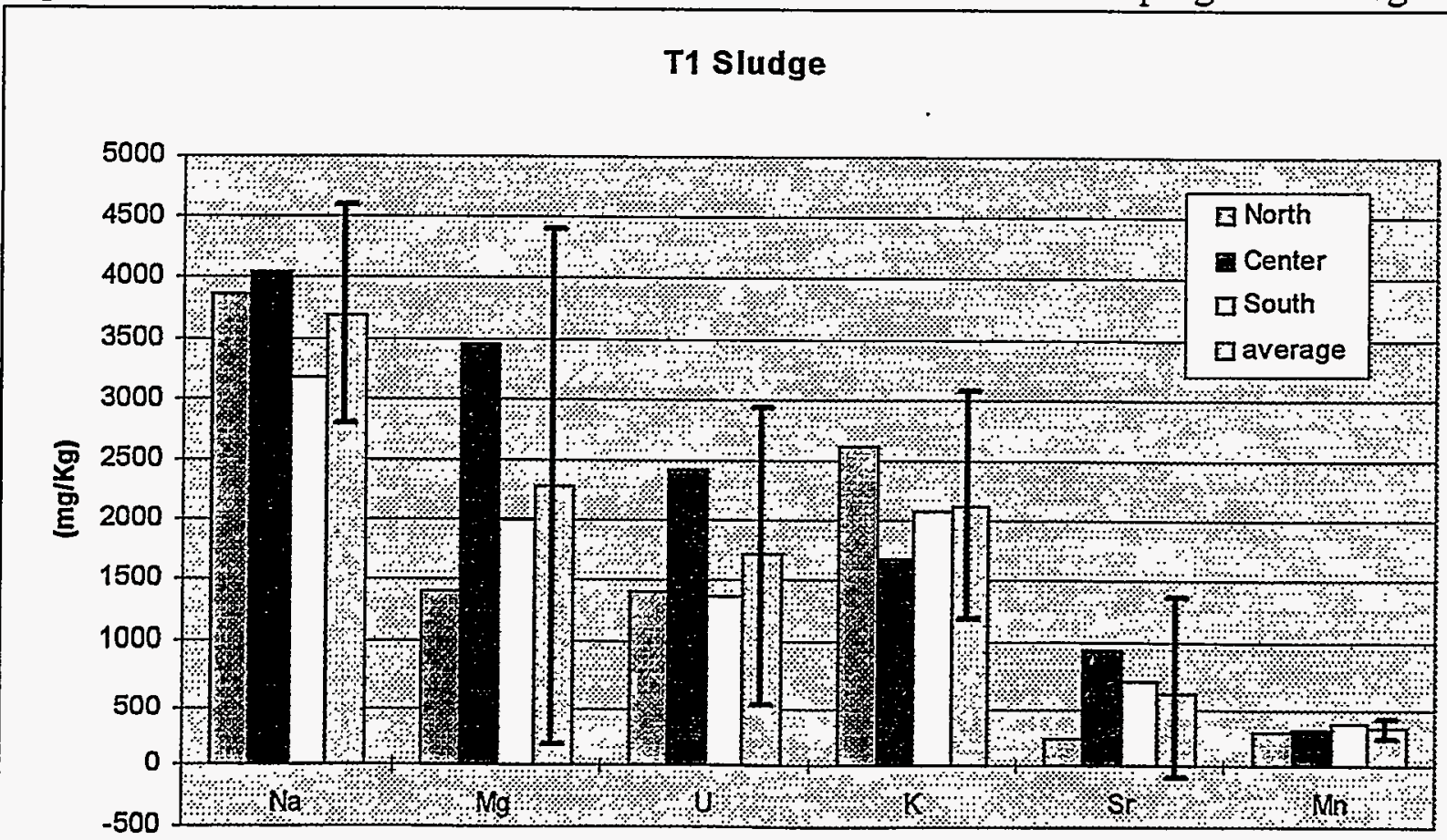


Figure 29 Lateral Distribution of Major Metals in T2 Sludge

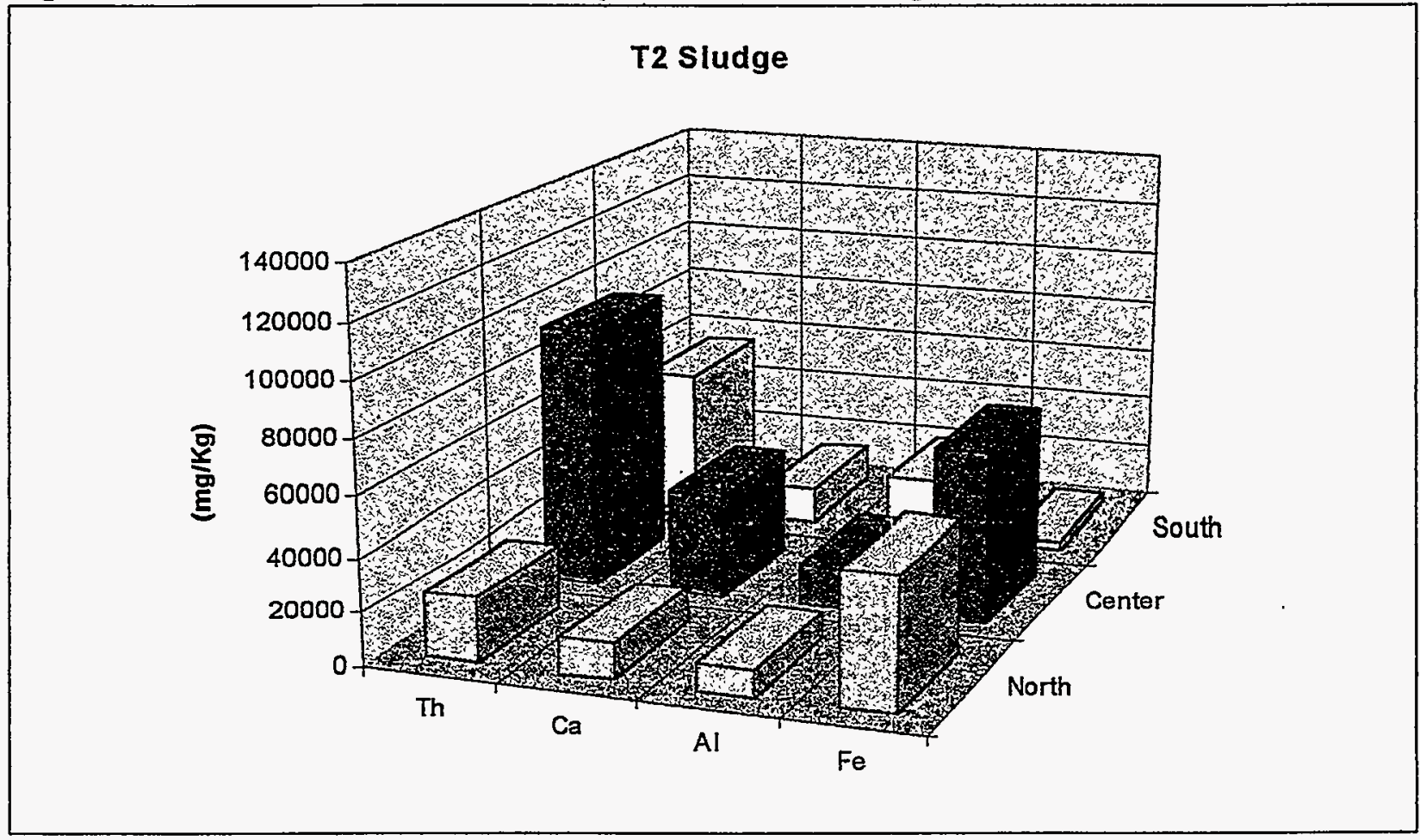

Figure $30 \quad$ Lateral Distribution of Selected Metals in T2 Sludge

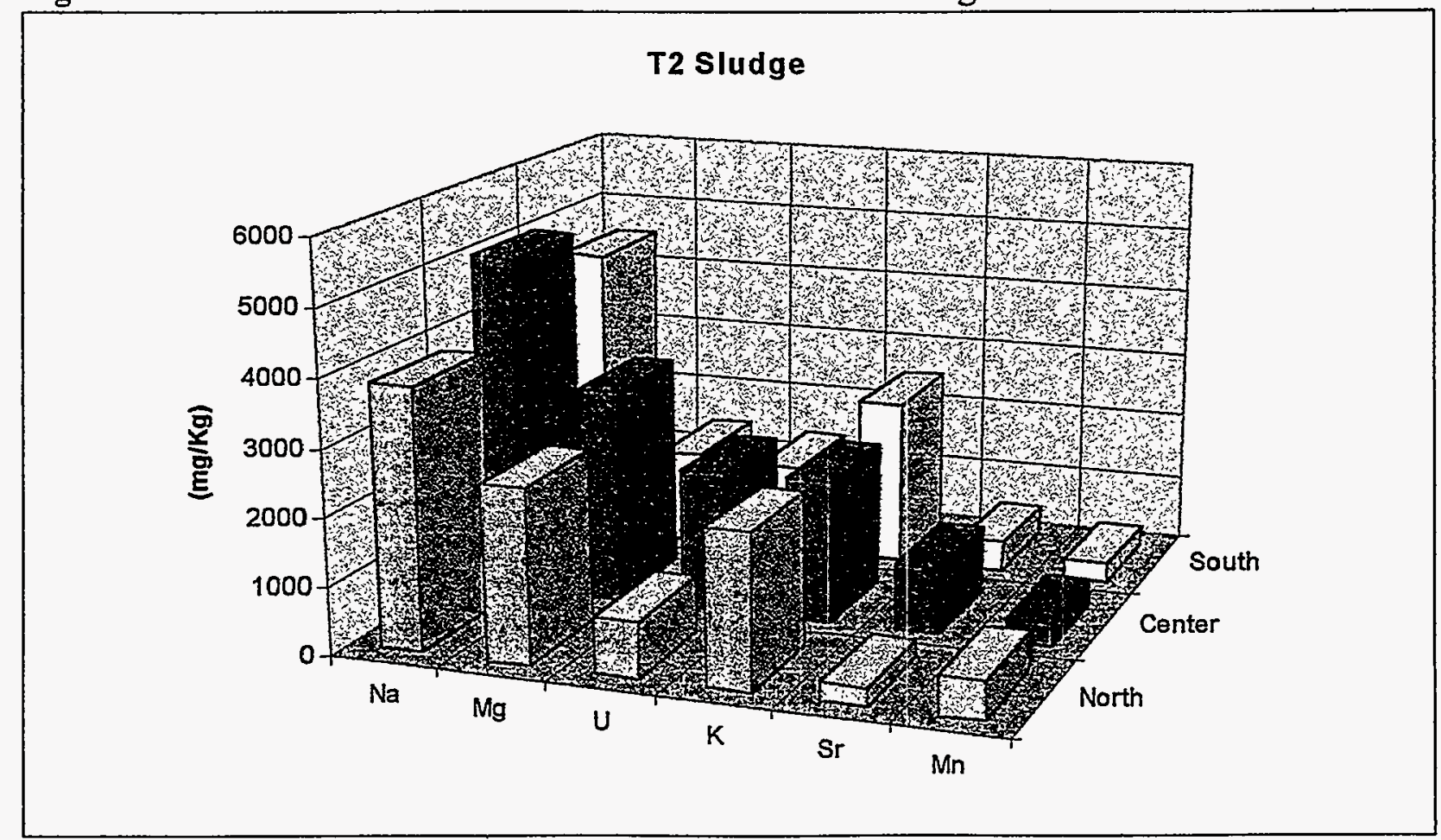


Figure 31 Statistical Distribution of Major Metals from Lateral Sampling of T2 Sludge

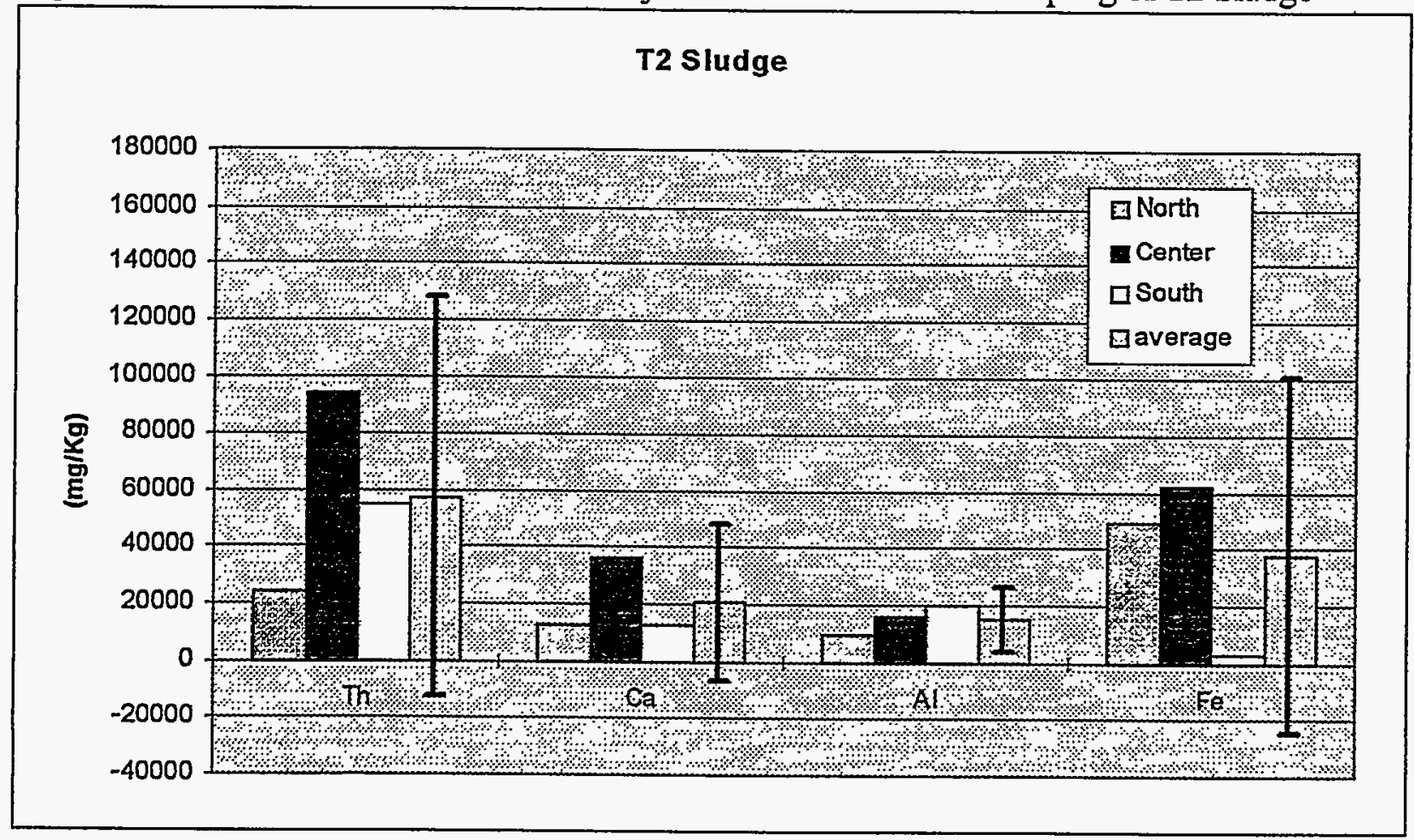

Figure 32 Statistical Distribution of Selected Metals from Lateral Sampling of T2 Sludge

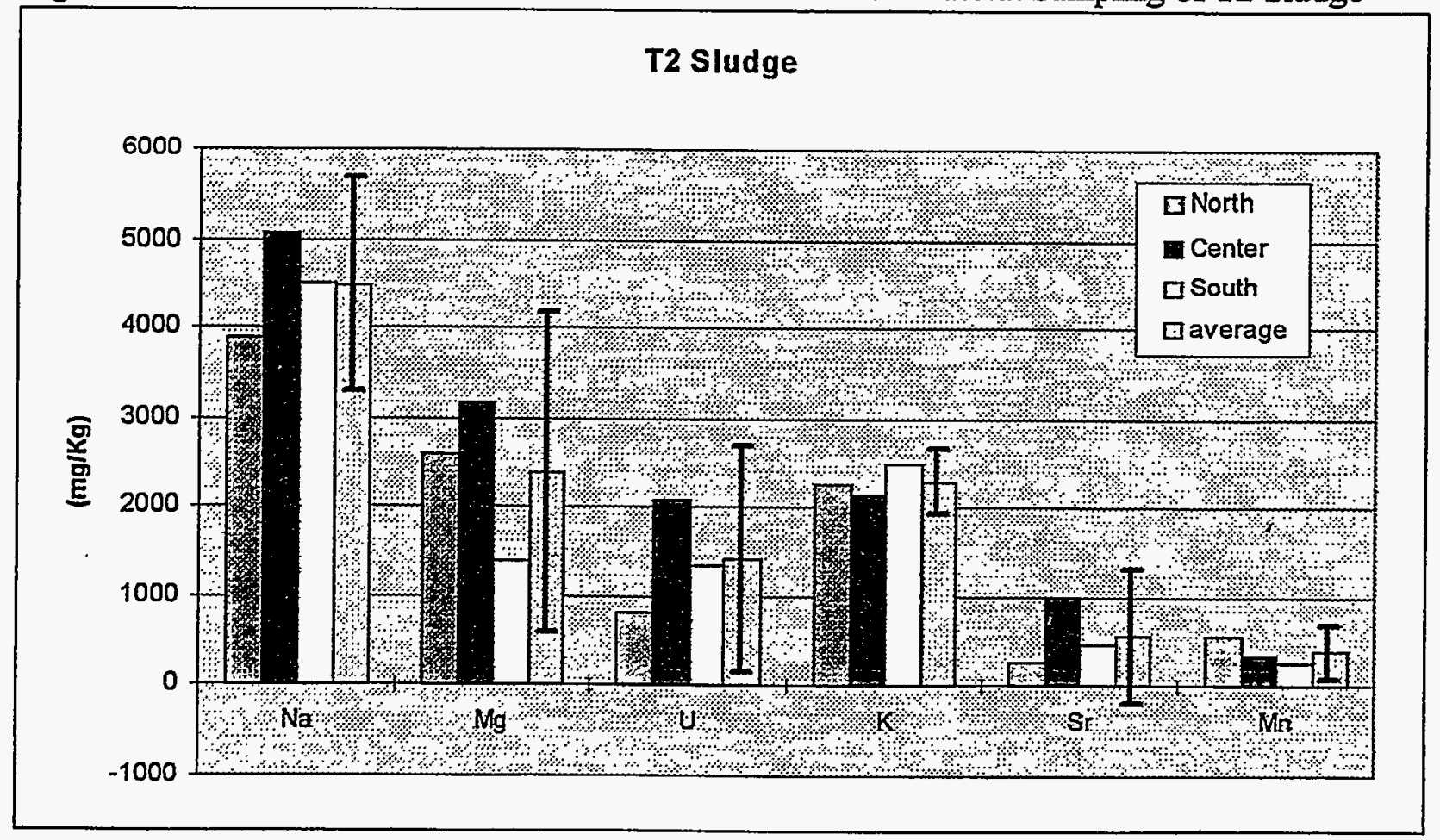


Figure 33 Lateral Distribution of Major Metals in T3 Sludge

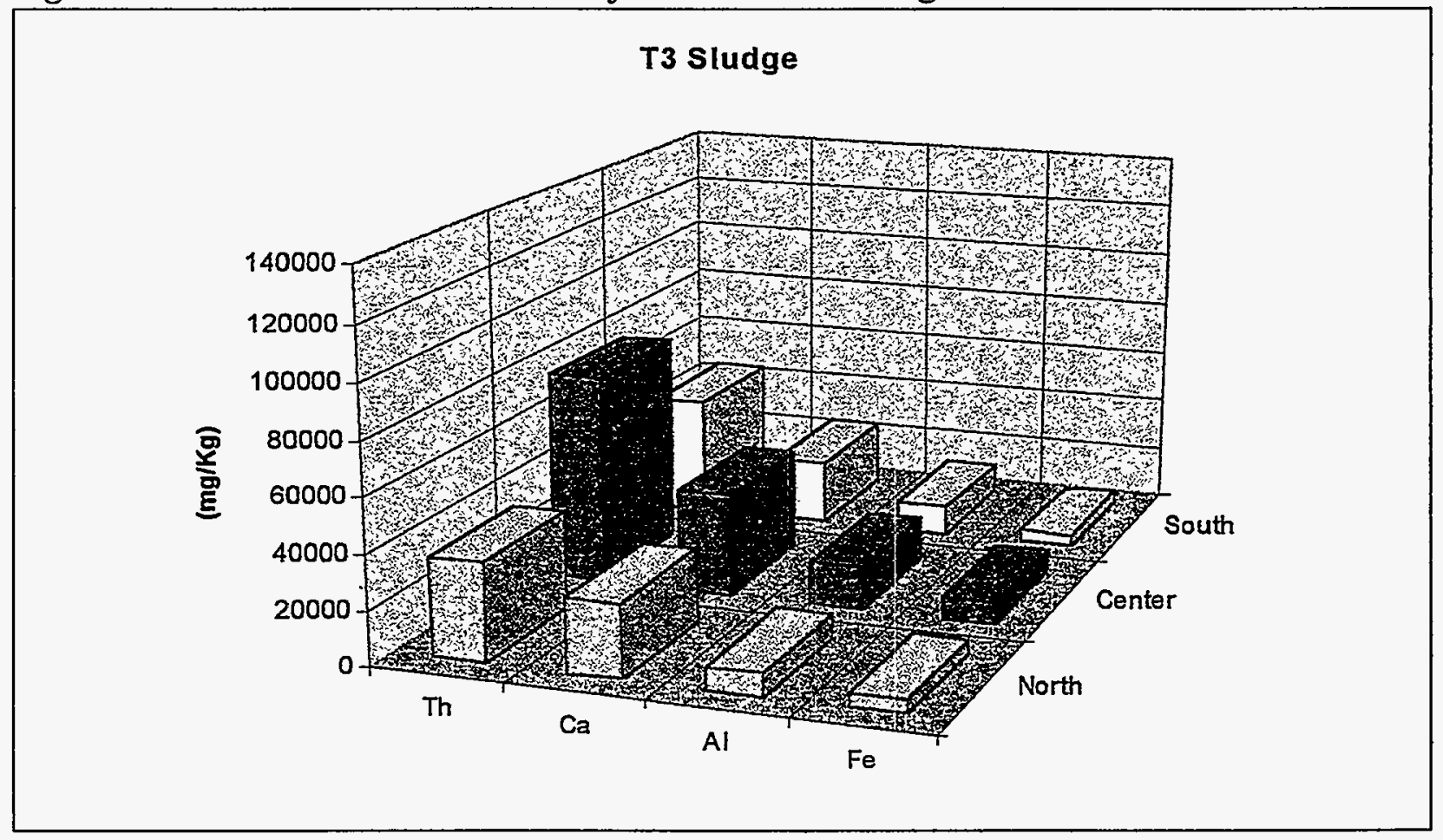

Figure 34 Lateral Distribution of Selected Metals in T3 Sludge

T3 Sludge

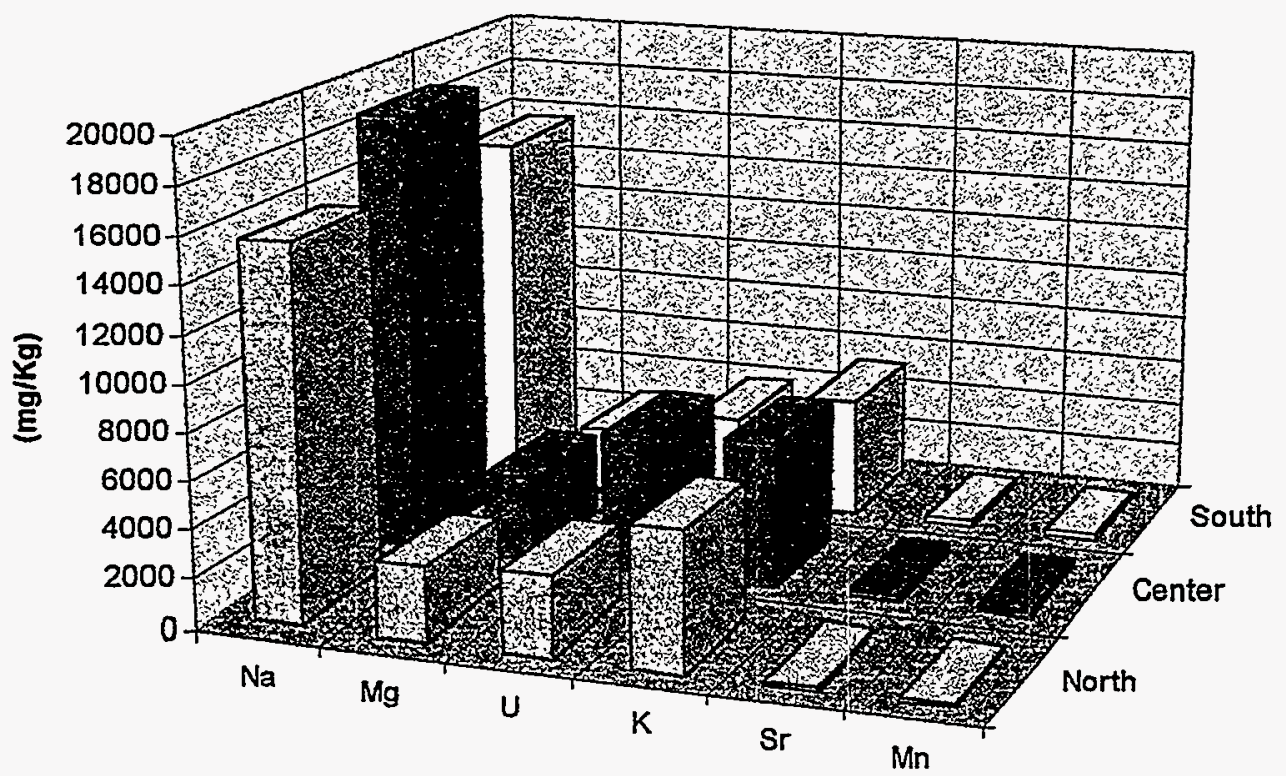


Figure 35 Statistical Distribution of Major Metals from Lateral Sampling of T3 Sludge

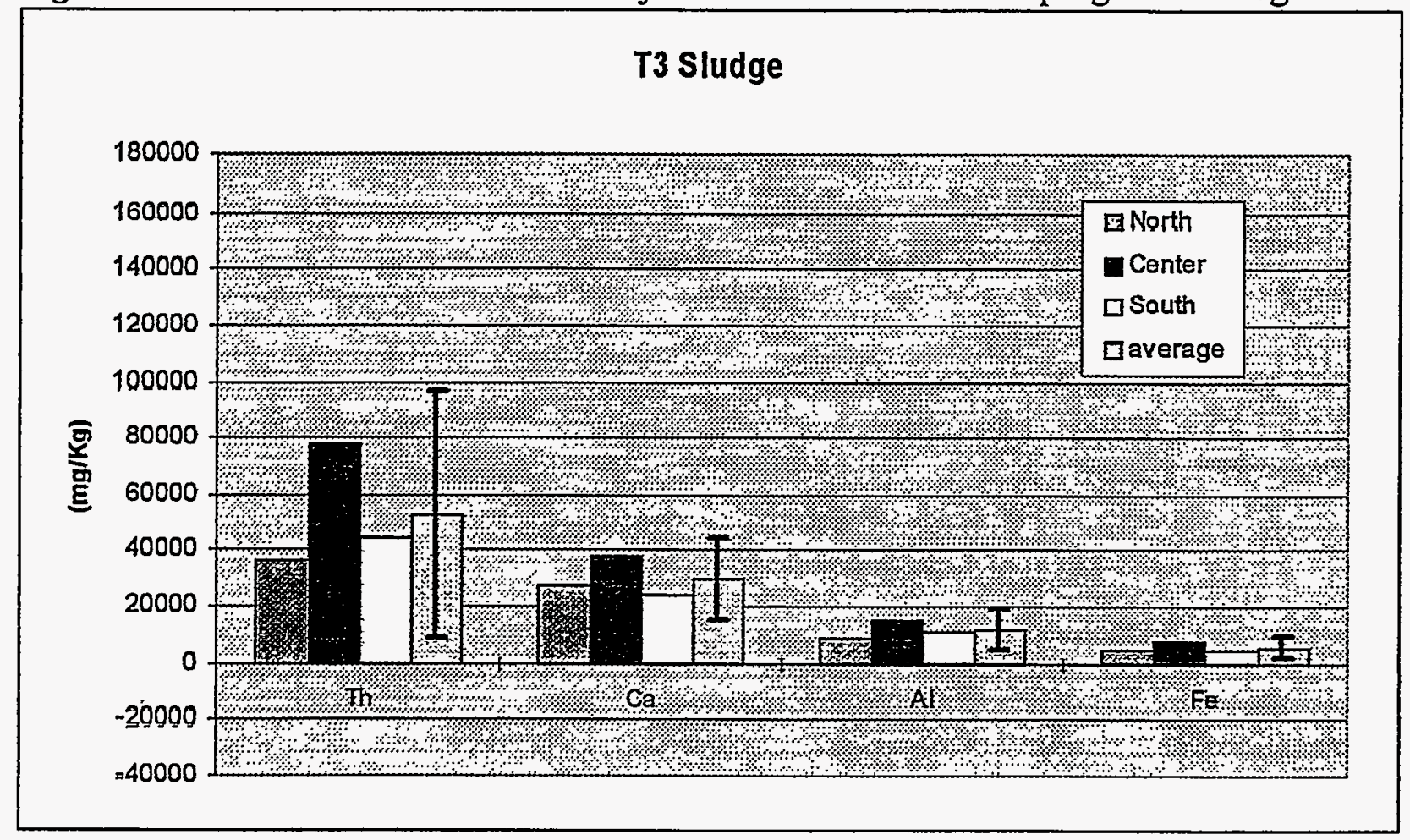

Figure 36 Statistical Distribution of Selected Metals from Lateral Sampling of T3 Sludge

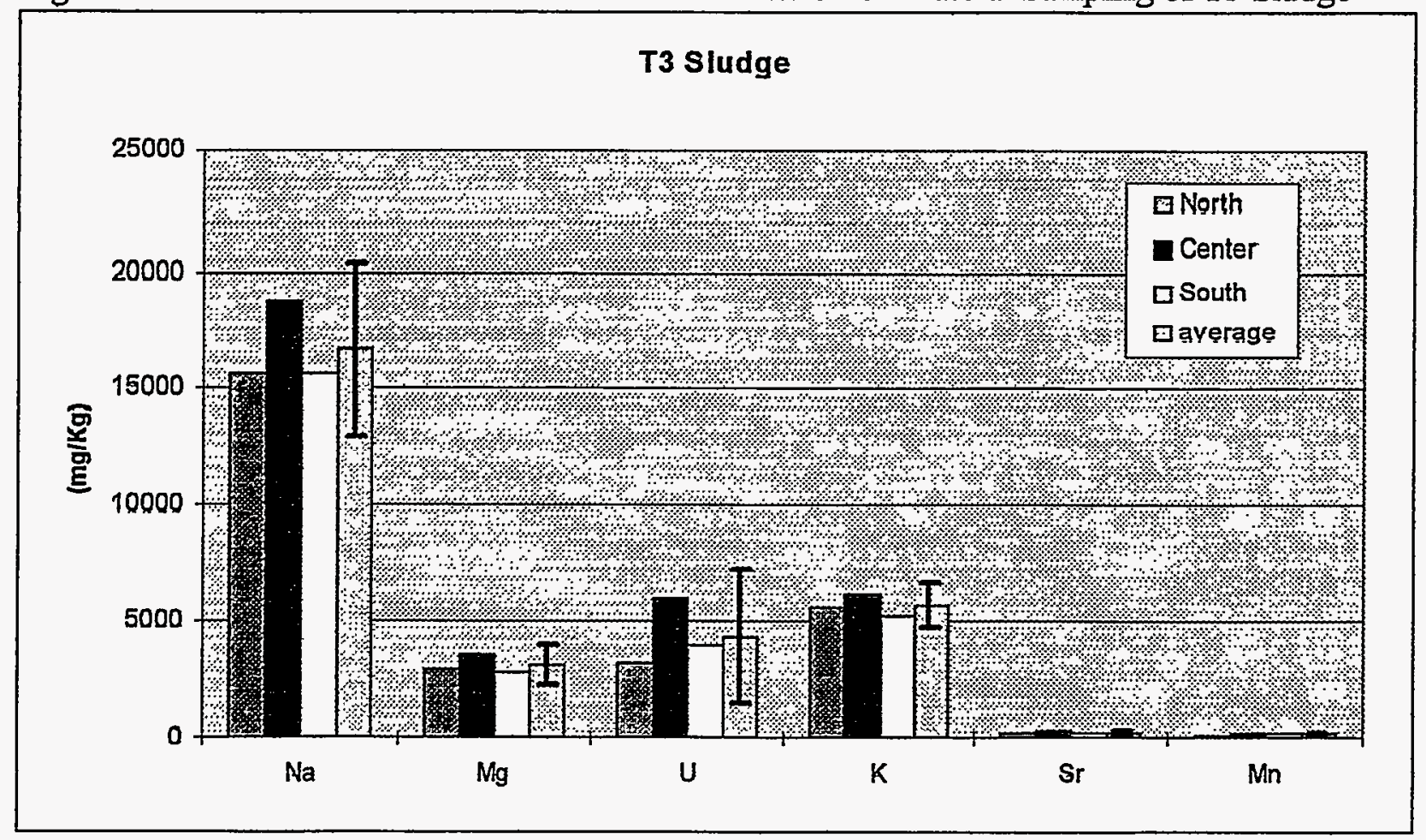


Figure 37 Lateral Distribution of Major Metals in T4 Sludge

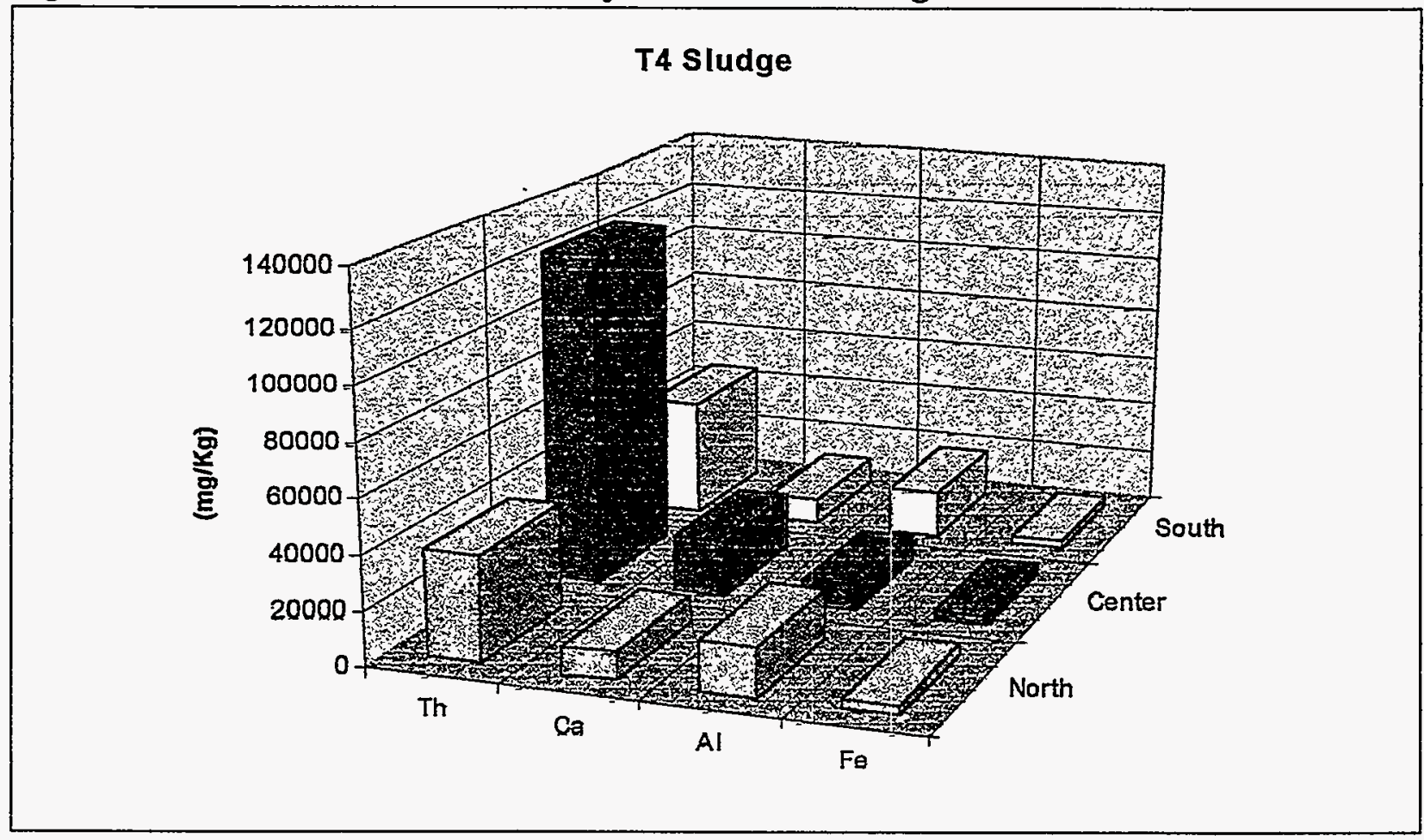

Figure $38 \quad$ Lateral Distribution of Selected Metals in T4 Sludge

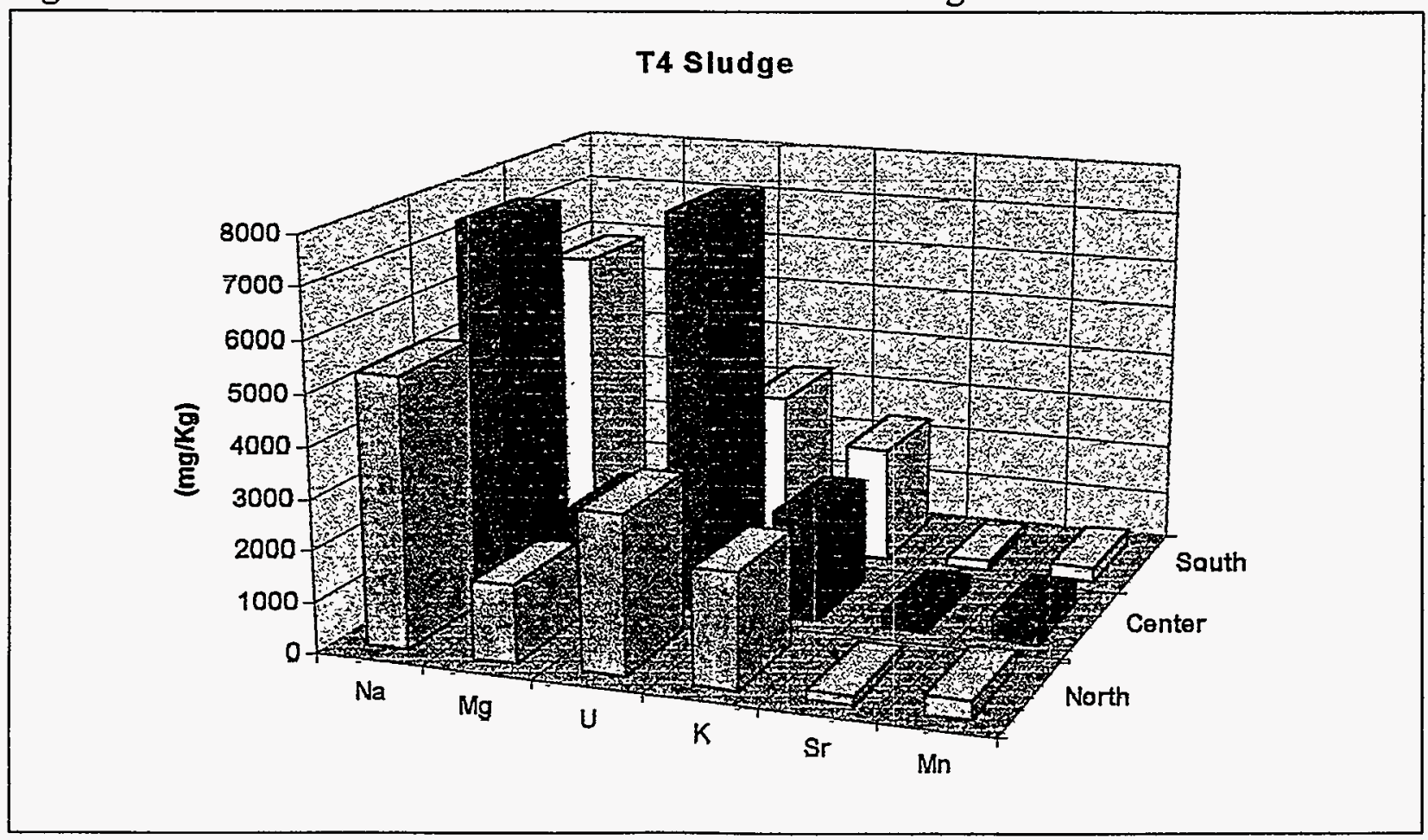


Figure 39 Statistical Distribution of Major Metals from Lateral Sampling of T4 Sludge

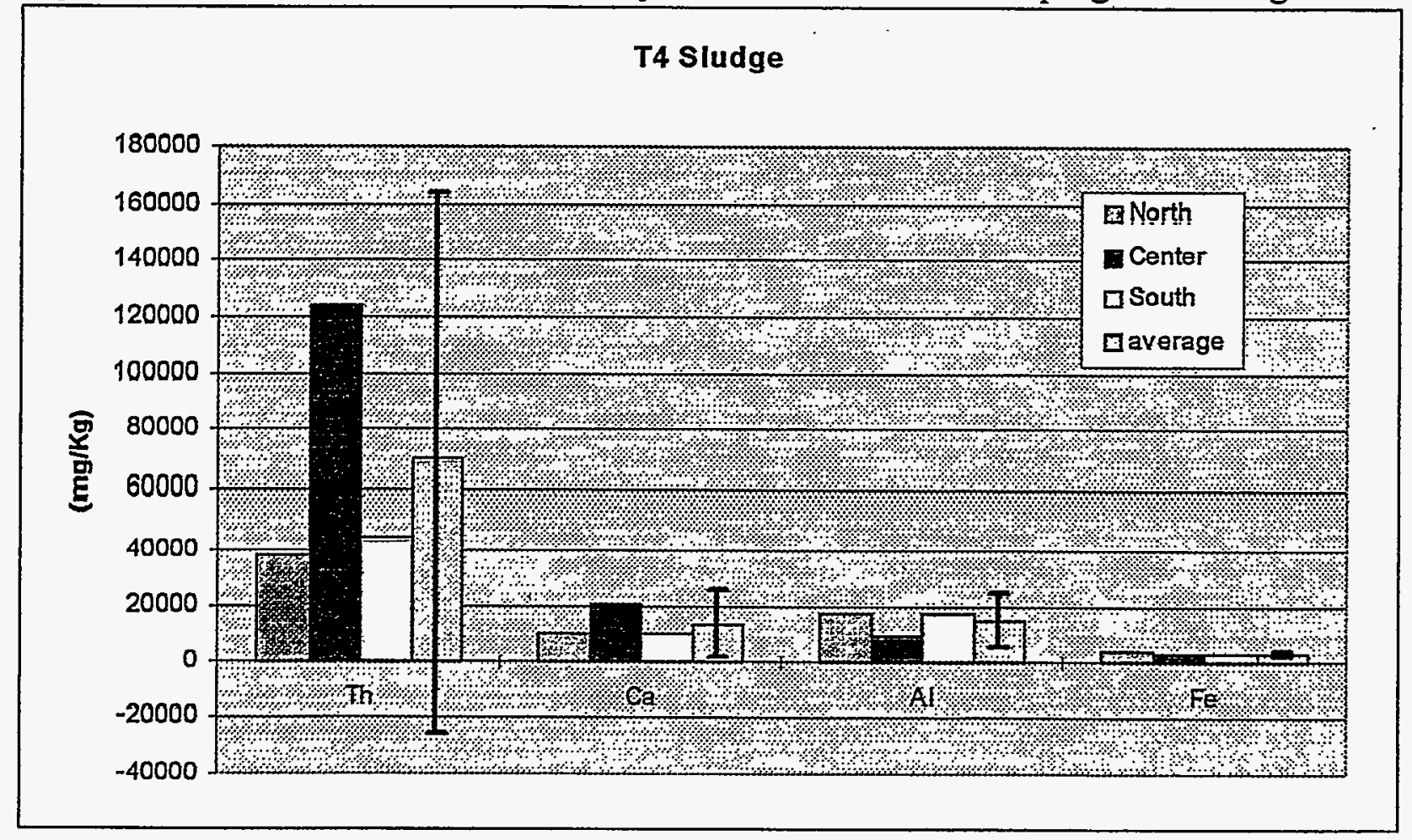

Figure 40 Statistical Distribution of Selected Metals from Lateral Sampling of T4 Sludge

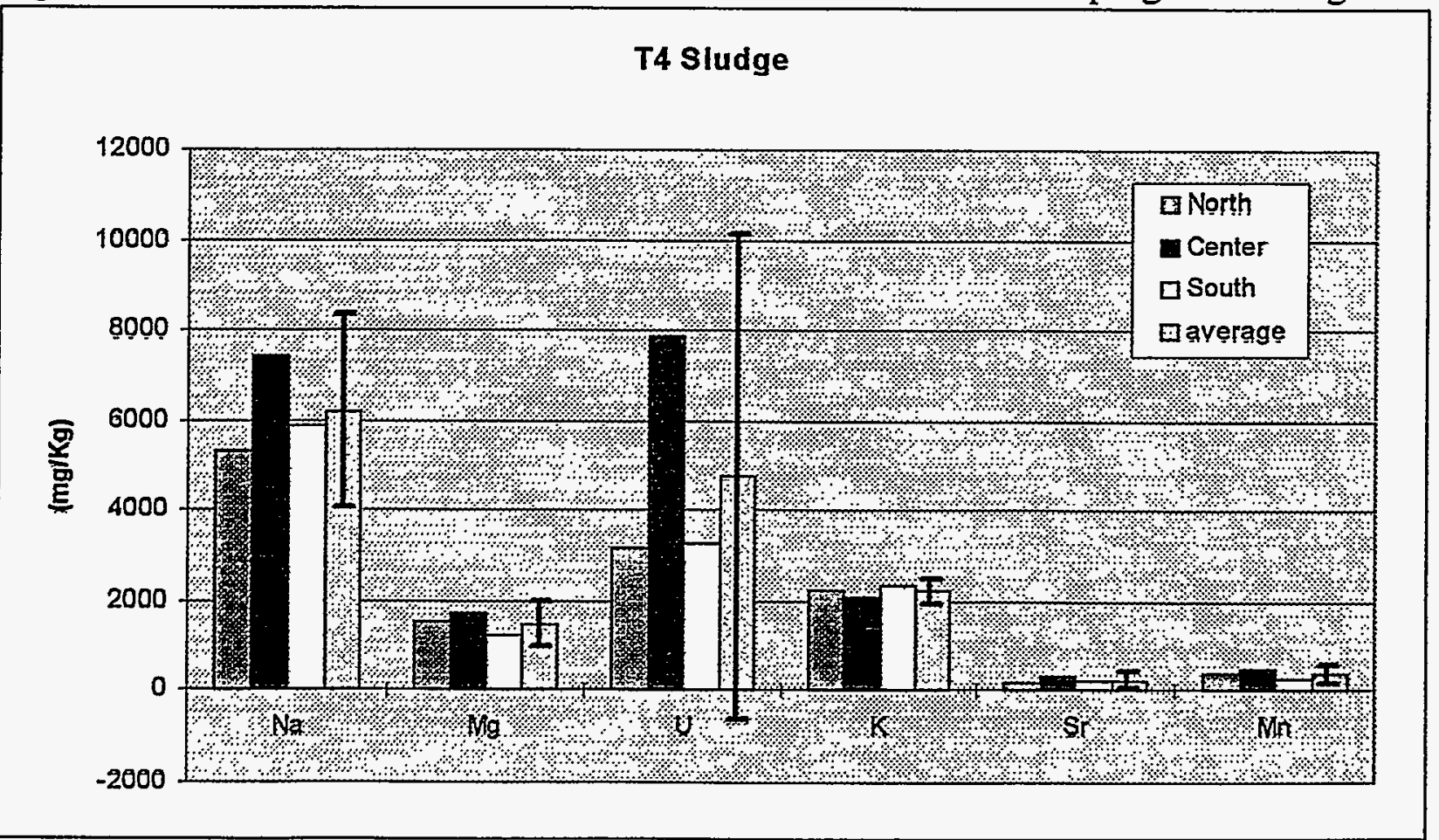


Figure 41 Lateral Distribution of Major Metals in T9 Sludge

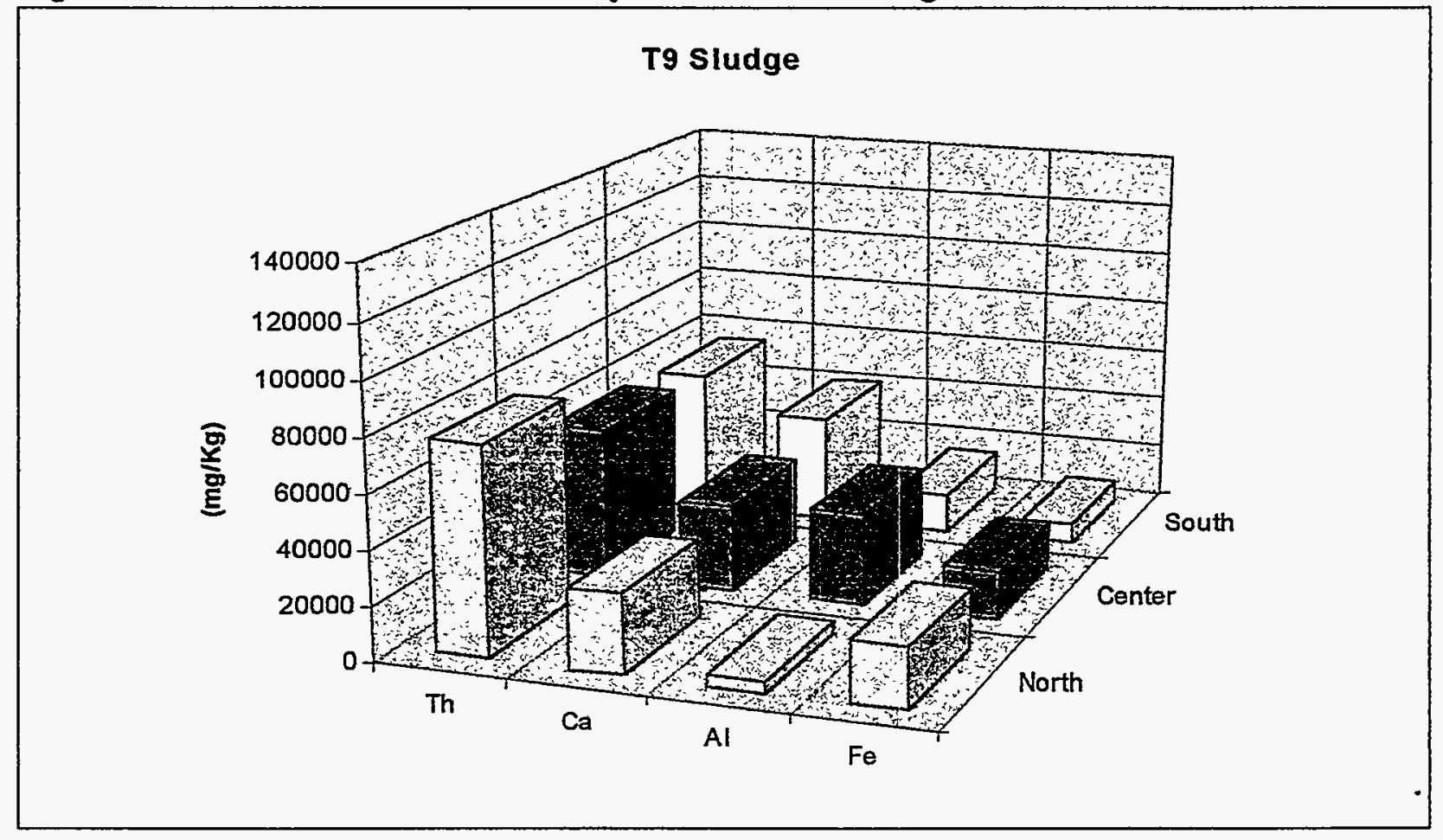

Figure 42 Lateral Distribution of Selected Metals in T9 Sludge

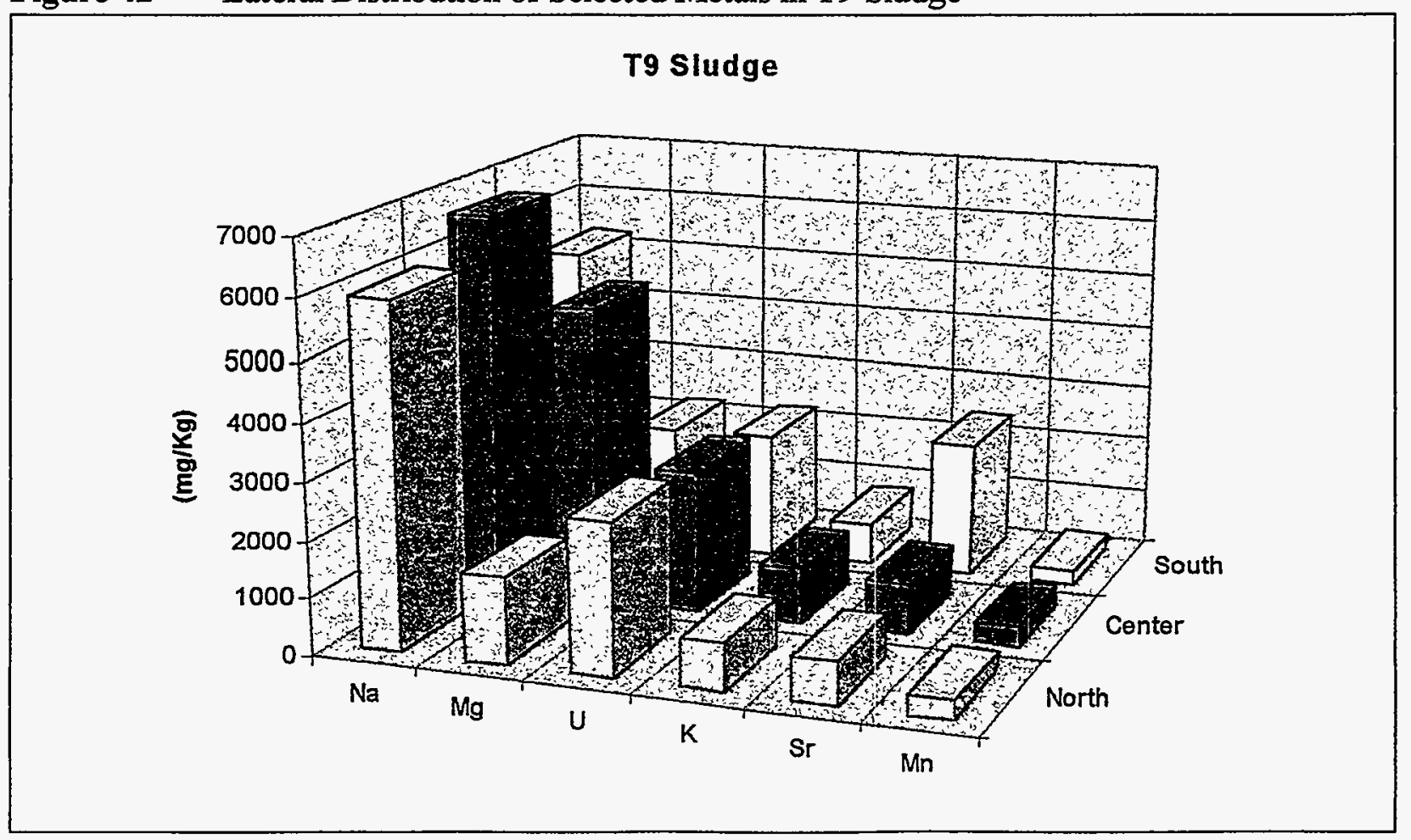


Figure 43 Statistical Distribution of Major Metals from Lateral Sampling of T9 Sludge

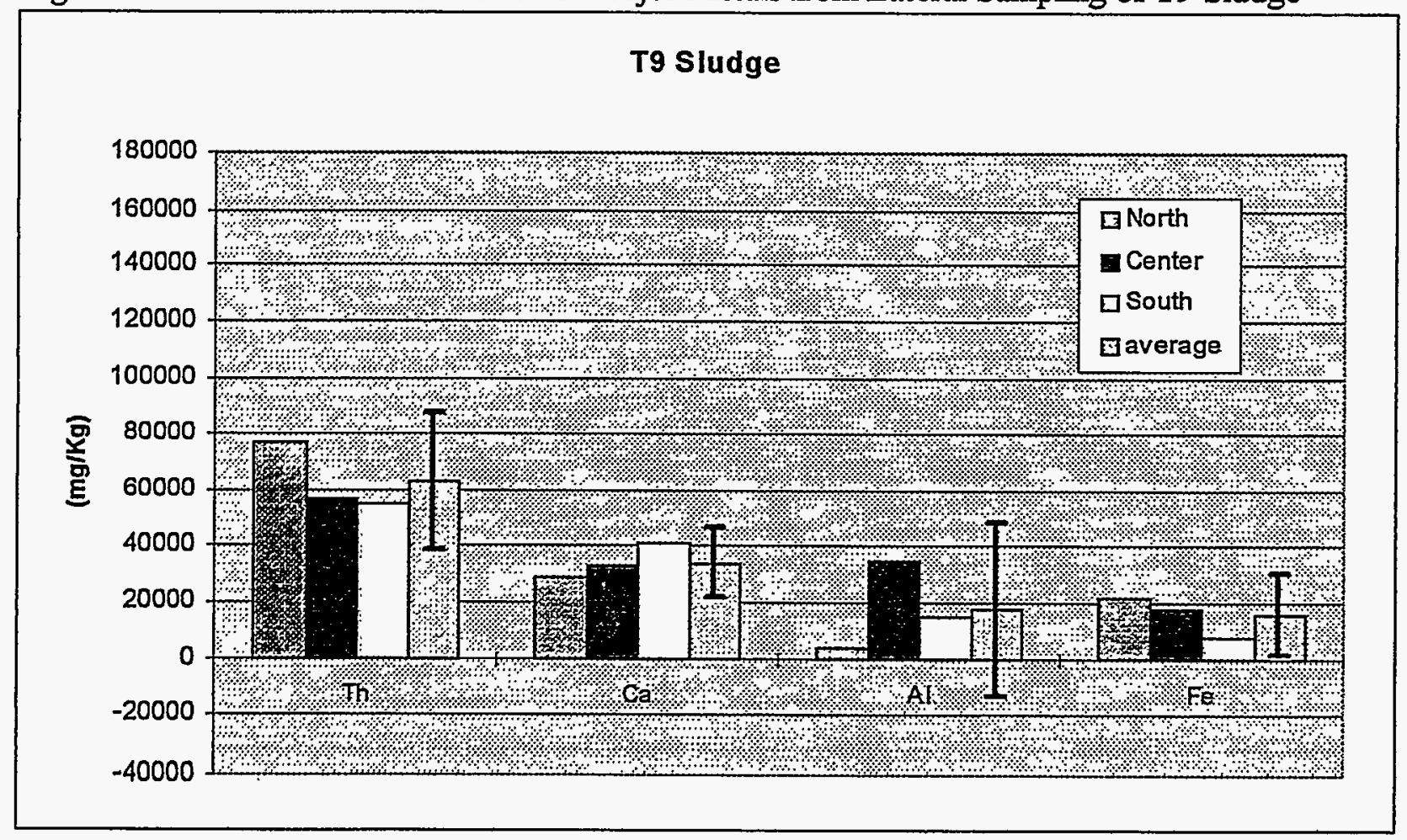

Figure 44 Statistical Distribution of Selected Metals from Lateral Sampling of T9 Sludge T9 sludge

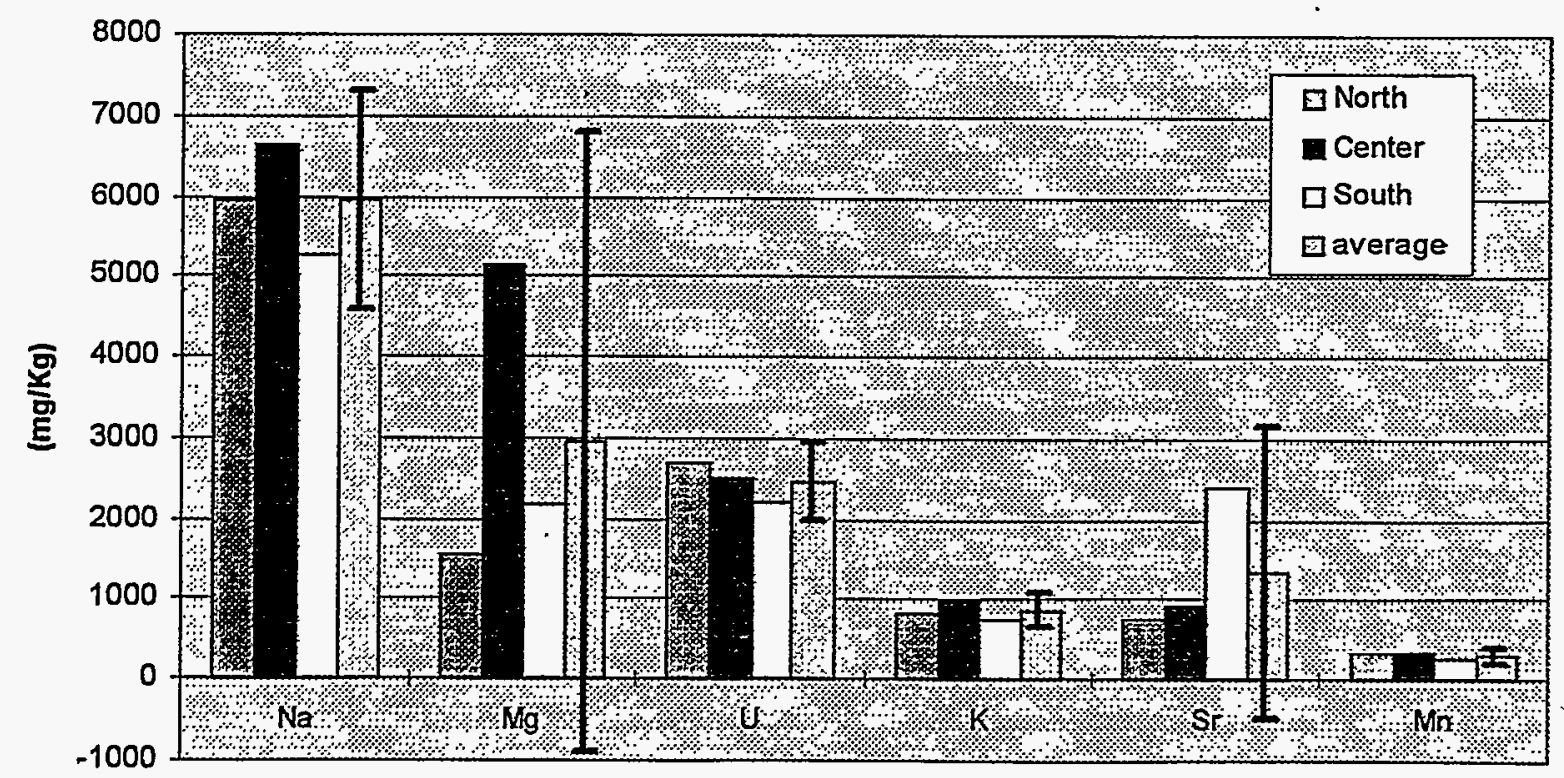




\subsection{Statistical summary for the major radionuclides}

A summary of the activities for the major radionuclides observed at each sample location are tabulated for each tank in Tables $43-47$, along with the average, $2 \sigma$ error, and the $2 \sigma$ percent relative error. The same information for the selected radionuclides across all five tanks collectively is provide in Table 48. 
Table 43 Summary statistics for major radionuclides in T1 sludge

\begin{tabular}{|c|c|c|c|c|c|c|}
\hline \multirow[b]{2}{*}{ Element } & \multicolumn{6}{|c|}{$\begin{array}{c}\mathrm{T} 1 \\
(\mathrm{~Bq} / \mathrm{g})\end{array}$} \\
\hline & North & Center & South & Mean & $2 \sigma$ & $\% 20$ \\
\hline${ }^{60} \mathrm{Co}$ & $4.90 E+04$ & $6.70 E+04$ & $2.40 \mathrm{E}+04$ & $4.67 E+04$ & $4.32 E+04$ & $92.5 \%$ \\
\hline${ }^{90} \mathrm{Sr}$ & $6.60 E \div 06$ & $2.00 \mathrm{E}+07$ & $1.30 \mathrm{E}+07$ & 1.32E+07 & $1.34 E+07$ & $101.5 \%$ \\
\hline${ }^{137} \mathrm{Cs}$ & $3.90 E+05$ & $4.50 E+05$ & $2.90 E+05$ & $3.77 E+05$ & $1.62 E+05$ & $42.9 \%$ \\
\hline${ }^{152} \mathrm{Eu}$ & 4.30E+04 & $6.30 E+04$ & $2.30 \mathrm{E}+04$ & $4.30 \mathrm{E}+04$ & $4.00 E+04$ & $93.0 \%$ \\
\hline${ }^{233} \mathrm{U}$ & $6.10 E+03$ & $7.90 E+03$ & $3.00 E+03$ & $5.67 \mathrm{E}+03$ & $4.96 E+03$ & $87.5 \%$ \\
\hline${ }^{238} \mathrm{Pu}$ & $9.70 E+03$ & $2.90 E+04$ & $1.60 E+04$ & $1.82 E+04$ & $1.97 E+04$ & $108.0 \%$ \\
\hline${ }^{239 / 240} \mathrm{Pu}$ & $4.90 E+03$ & $1.10 \mathrm{E}+04$ & $5.80 \mathrm{E}+03$ & $7.23 E+03$ & $6.59 E+03$ & $91.0 \%$ \\
\hline${ }^{241} \mathrm{Am}$ & 1.10E+04 & $5.20 E+04$ & $1.70 E+04$ & $2.67 E+04$ & 4.43E+04 & $166.1 \%$ \\
\hline${ }^{244} \mathrm{Cm}$ & $2.30 \mathrm{E}+05$ & $3.50 E+05$ & $2.00 E+05$ & $2.60 \mathrm{E}+05$ & $1.59 E+05$ & $61.1 \%$ \\
\hline
\end{tabular}

Table 44 Summary statistics for major radionuclides in T2 sludge

\begin{tabular}{|c|c|c|c|c|c|c|}
\hline \multirow[b]{2}{*}{ Element } & \multicolumn{6}{|c|}{$\begin{array}{c}\mathrm{T} 2 \\
(\mathrm{~Bq} / \mathrm{g})\end{array}$} \\
\hline & North & Center & South & average & $2 \sigma$ & $\% 2 \sigma$ \\
\hline${ }^{60} \mathrm{Co}$ & $2.30 E+04$ & $7.70 E+04$ & $3.20 E \div 04$ & $4.40 E \div 04$ & $5.79 E+04$ & $131.5 \%$ \\
\hline${ }^{90} \mathrm{Sr}$ & $6.10 E+06$ & $1.80 E+07$ & $1.20 \mathrm{E} \div 07$ & $1.20 E+07$ & $1.19 E+07$ & $98.9 \%$ \\
\hline${ }^{137} \mathrm{Cs}$ & $2.10 E+05$ & $3.50 E+05$ & 2.30E+05 & $2.63 E+05$ & $1.51 E+05$ & $57.5 \%$ \\
\hline${ }^{152} \mathrm{Eu}$ & $1.90 E+04$ & $7.30 \mathrm{E}+04$ & $2.90 E+04$ & $4.03 E+04$ & $5.75 E+04$ & $142.5 \%$ \\
\hline${ }^{233} U$ & $2.80 E+03$ & $7.80 \mathrm{E}+03$ & 4.30E+03 & $4.97 E+03$ & $5.13 E+03$ & $103.3 \%$ \\
\hline${ }^{238} \mathrm{Pu}$ & $7.90 E+03$ & $2.30 E+04$ & $1.50 \mathrm{E}+04$ & $1.53 E+04$ & 1.51E+04 & $98.8 \%$ \\
\hline${ }^{239 / 240} \mathrm{Pu}$ & $3.80 E+03$ & $1.00 E+04$ & $7.20 E+03$ & $7.00 \mathrm{E}+03$ & $6.21 \mathrm{E}+03$ & $88.7 \%$ \\
\hline${ }^{241} \mathrm{Am}$ & $5.00 E+03$ & 2.60E+04 & $8.90 E+03$ & $1.33 E+04$ & $2.23 E+04$ & $168.0 \%$ \\
\hline${ }^{244} \mathrm{Cm}$ & $1.10 E+05$ & $4.60 \mathrm{E}+05$ & $2.20 \mathrm{E}+05$ & $2.63 E+05$ & $3.58 \mathrm{E}+05$ & $135.9 \%$ \\
\hline
\end{tabular}

Table 45 Summary statistics for major radionuclides in T3 sludge

\begin{tabular}{|ccccccr||}
\hline \hline & \multicolumn{7}{c|}{$\begin{array}{c}\text { T3 } \\
\text { (Bq/g) }\end{array}$} \\
\cline { 2 - 7 } Element & North & Center & South & average & 20 & $\% 20$ \\
\hline \hline${ }^{60} \mathrm{Co}$ & $4.50 \mathrm{E}+04$ & $1.00 \mathrm{E}+05$ & $5.50 \mathrm{E}+04$ & $6.67 \mathrm{E}+04$ & $5.86 \mathrm{E}+04$ & $87.9 \%$ \\
${ }^{90} \mathrm{Sr}$ & $4.70 \mathrm{E}+06$ & $8.50 \mathrm{E}+06$ & $7.50 \mathrm{E}+06$ & $6.90 \mathrm{E}+06$ & $3.94 \mathrm{E}+06$ & $57.1 \%$ \\
${ }^{137} \mathrm{Cs}$ & $1.10 \mathrm{E}+06$ & $1.60 \mathrm{E}+06$ & $9.90 \mathrm{E}+05$ & $1.23 \mathrm{E}+06$ & $6.50 \mathrm{E}+05$ & $52.9 \%$ \\
${ }^{152} \mathrm{Eu}$ & $3.00 \mathrm{E}+04$ & $5.60 \mathrm{E}+04$ & $4.00 \mathrm{E}+04$ & $4.20 \mathrm{E}+04$ & $2.62 \mathrm{E}+04$ & $62.5 \%$ \\
\hline${ }^{233} \mathrm{U}$ & $7.70 \mathrm{E}+03$ & $1.50 \mathrm{E}+04$ & $9.90 \mathrm{E}+03$ & $1.09 \mathrm{E}+04$ & $7.49 \mathrm{E}+03$ & $68.9 \%$ \\
${ }^{238} \mathrm{Pu}$ & $9.80 \mathrm{E}+03$ & $1.70 \mathrm{E}+04$ & $1.50 \mathrm{E}+04$ & $1.39 \mathrm{E}+04$ & $7.43 \mathrm{E}+03$ & $53.3 \%$ \\
${ }^{239240} \mathrm{Pu}$ & $3.60 \mathrm{E}+03$ & $6.50 \mathrm{E}+03$ & $4.20 \mathrm{E}+03$ & $4.77 \mathrm{E}+03$ & $3.06 \mathrm{E}+03$ & $64.2 \%$ \\
${ }^{241} \mathrm{Am}$ & $2.00 \mathrm{E}+03$ & $1.50 \mathrm{E}+04$ & $1.40 \mathrm{E}+04$ & $1.03 \mathrm{E}+04$ & $1.45 \mathrm{E}+04$ & $140.0 \%$ \\
${ }^{244} \mathrm{Cm}$ & $1.40 \mathrm{E}+05$ & $2.50 \mathrm{E}+05$ & $1.90 \mathrm{E}+05$ & $1.93 \mathrm{E}+05$ & $1.10 \mathrm{E}+05$ & $57.0 \%$ \\
\hline
\end{tabular}


Table 46 Summary statistics for major radionuclides in T4 sludge

\begin{tabular}{|c|c|c|c|c|c|c|}
\hline \multirow[b]{2}{*}{ Element } & \multicolumn{6}{|c|}{$\begin{array}{c}\mathrm{T} 4 \\
(\mathrm{~Bq} / \mathrm{g})\end{array}$} \\
\hline & North & Center & South & average & $2 \sigma$ & $\% 2 \sigma$ \\
\hline${ }^{60} \mathrm{Co}$ & $1.90 \mathrm{E}+04$ & $1.60 \mathrm{E}+05$ & $3.40 E+04$ & $7.10 E+04$ & $1.55 E+05$ & $218.1 \%$ \\
\hline${ }^{90} \mathrm{Sr}$ & $1.20 \mathrm{E}+07$ & 1.60E+07 & $1.40 E+07$ & $1.40 E+07$ & $4.00 \mathrm{E}+06$ & $28.6 \%$ \\
\hline${ }^{137} \mathrm{Cs}$ & $2.90 E+05$ & $3.40 E+05$ & $2.90 E+05$ & $3.07 E+05$ & $5.77 E+04$ & $18.8 \%$ \\
\hline${ }^{152} \mathrm{Eu}$ & $3.40 \mathrm{E}+04$ & 1.20E $\div 05$ & $4.00 E+04$ & $6.47 E+04$ & $9.60 \mathrm{E}+04$ & $148.5 \%$ \\
\hline${ }^{233} \mathrm{U}$ & $5.40 \mathrm{E}+03$ & $2.40 E+04$ & $6.90 \mathrm{E}+03$ & $1.21 E+04$ & $2.07 E+04$ & $170.8 \%$ \\
\hline${ }^{238} \mathrm{Pu}$ & $2.70 E+04$ & $2.20 E+04$ & $2.60 E+04$ & $2.50 \mathrm{E}+04$ & $5.29 E \div 03$ & $21.2 \%$ \\
\hline${ }^{239 / 240} \mathrm{Pu}$ & $5.20 E+03$ & $1.30 E+04$ & $6.30 E+03$ & 8.17E+03 & $8.44 E+03$ & $103.4 \%$ \\
\hline${ }^{241} \mathrm{Am}$ & $1.60 \mathrm{E}+04$ & $8.00 E+03$ & $2.30 E+04$ & $1.57 E+04$ & $1.50 E+04$ & $95.8 \%$ \\
\hline${ }^{244} \mathrm{Cm}$ & $2.50 E+05$ & $5.30 \mathrm{E}+05$ & $2.90 \mathrm{E}+05$ & $3.57 \mathrm{E}+05$ & $3.03 E+05$ & $84.9 \%$ \\
\hline
\end{tabular}

Table 47 Summary statistics for major radionuclides in T9 sludge

\begin{tabular}{|c|c|c|c|c|c|c|}
\hline \multirow[b]{2}{*}{ Element } & \multicolumn{6}{|c|}{$\begin{array}{c}\mathrm{T} 9 \\
(\mathrm{~Bq} / \mathrm{g})\end{array}$} \\
\hline & North. & Center & South & average & $2 \sigma$ & $\% 2 \sigma$ \\
\hline${ }^{60} \mathrm{Co}$ & $8.40 E+04$ & $4.90 E+04$ & $5.90 E+04$ & $6.40 E+04$ & $3.61 E+04$ & $56.3 \%$ \\
\hline${ }^{50} \mathrm{Sr}$ & $1.00 E+07$ & $2.00 E+07$ & $1.50 E+07$ & $1.50 E+07$ & $1.00 E+07$ & $66.7 \%$ \\
\hline${ }^{137} \mathrm{Cs}$ & $1.90 E+05$ & $2.60 E+05$ & $2.10 E+05$ & $2.20 E+05$ & $7.21 E+04$ & $32.8 \%$ \\
\hline${ }^{152} \mathrm{Eu}$ & $6.50 E+04$ & $4.30 E+04$ & $4.50 E+04$ & $5.10 E+04$ & $2.43 E+04$ & $47.7 \%$ \\
\hline${ }^{233} \mathrm{U}$ & $1.10 E+04$ & $6.40 E+03$ & $7.30 E+03$ & $8.23 E+03$ & $4.88 E+03$ & $59.2 \%$ \\
\hline${ }^{238} \mathrm{Pu}$ & $1.20 E+04$ & $4.80 \mathrm{E}+04$ & $2.40 E+04$ & $2.80 E+04$ & $3.67 E+04$ & $130.9 \%$ \\
\hline${ }^{239 / 240} \mathrm{Pu}$ & $7.40 \mathrm{E}+03$ & $9.20 E+03$ & $7.40 E+03$ & $8.00 E+03$ & $2.08 E+03$ & $26.0 \%$ \\
\hline${ }^{241} \mathrm{Am}$ & $3.40 E+04$ & $1.30 \mathrm{E}+04$ & $9.00 E+03$ & 1.87E+04 & $2.69 E+04$ & $143.9 \%$ \\
\hline${ }^{244} \mathrm{Cm}$ & $2.80 E+05$ & $2.70 E+05$ & $2.70 E+05$ & $2.73 E+05$ & $1.15 \mathrm{E}+04$ & $4.2 \%$ \\
\hline
\end{tabular}

Table 48 Summary statistics for major radionuclides in T1-T9 sludge

\begin{tabular}{|c|c|c|c|c|c|c|}
\hline \multirow[b]{2}{*}{ Element } & \multicolumn{6}{|c|}{$\begin{array}{l}\text { T1-T9 } \\
(\mathrm{Bq} / \mathrm{g})\end{array}$} \\
\hline & North & Center & South & average & 20 & $\% 2 \sigma$ \\
\hline${ }^{60} \mathrm{Co}$ & $4.40 E+04$ & $9.06 E+04$ & $4.08 E+04$ & $5.85 E+04$ & $5.57 \mathrm{E}+04$ & $95.4 \%$ \\
\hline${ }^{90} \mathrm{Sr}$ & $7.88 E+06$ & $1.65 \mathrm{E}+07$ & $1.23 E+07$ & $1.22 E+07$ & $8.62 E+06$ & $70.5 \%$ \\
\hline${ }^{137} \mathrm{Cs}$ & $4.36 E+05$ & $6.00 E+05$ & $4.02 E+05$ & 4.79E+05 & $2.12 E+05$ & $44.2 \%$ \\
\hline${ }^{152} \mathrm{Eu}$ & $3.82 E+04$ & $7.10 E+04$ & $3.54 \mathrm{E}+04$ & $4.82 E+04$ & $3.96 \mathrm{E}+04$ & $82.1 \%$ \\
\hline${ }^{233} U$ & $6.60 E+03$ & $1.22 E+04$ & $6.28 E+03$ & 8.37E+03 & $6.68 \mathrm{E}+03$ & $79.9 \%$ \\
\hline${ }^{238} \mathrm{Pu}$ & 1.33E+04 & $2.78 E+04$ & $1.92 E+04$ & $2.01 E+04$ & $1.46 \mathrm{E}+04$ & $72.7 \%$ \\
\hline${ }^{239 / 240} \mathrm{Pu}$ & $4.98 E+03$ & $9.94 E+03$ & $6.18 E+03$ & $7.03 E+03$ & $5.18 E+03$ & $73.6 \%$ \\
\hline${ }^{241} \mathrm{Am}$ & $1.36 E+04$ & $2.28 E+04$ & $1.44 E+04$ & $1.69 E+04$ & $1.02 E+04$ & $60.3 \%$ \\
\hline${ }^{244} \mathrm{Cm}$ & $2.02 E+05$ & $3.72 E+05$ & $2.34 E+05$ & $2.69 E+05$ & $1.81 E+05$ & $67.1 \%$ \\
\hline
\end{tabular}




\section{REFERENCES}

1. LMES 1995 (Lockheed Martin Energy Systems, Inc.) ORNL Inactive Waste Tanks Sampling and Analysis Plan, ORNL RAP/LTR-88/24 (1988); Addendum 2: Old Hydrofracture Facility Tanks Sampling and Analysis Plan, December 1995.

2. LMES 1996 (Lockheed Martin Energy Systems, Inc.) ORNL Inactive Waste Tanks Sampling and Analysis Plan, ORNL RAP/LTR-88/24 (1988); Addendum 3: Old Hydrofracture Facility Tanks Sampling and Analysis Plan, November 1996.

3. J. W. Autrey, D. A. Costanzo, W. H. Griest, L. L. Kaiser, J. M. Keller, C. E. Nix, B. A. Tomkins, Sampling and Analysis of the Inactive Storage Tank Contents at ORNL, ORNL/ER-13, September 1990.

4. G. F. Boris, Lockheed Martin Energy Systems, Central Engineering, Oak Ridge, TN, personal communication to J. M. Keller, April 7, 1997.

5. U. S. Environmental Protection Agency, Test Methods for Evaluating Solid Waste, SW-846, 3rd ed, Office of Solid Waste and Emergency Response, Washington, D.C., November 1986; Update I, July 1992; and Final Update II, September 1994.

6. J. M. Giaquinto, A. M. Essling, and J. M. Keller, Comparison of SW-846 Method 3051 and SW-846 Method 7471A for the Preparation of Solid Waste Samples for Mercury Determination, ORNL/TM-13236, July 1996.

7. M. B. Sears, J. L. Botts, R. N. Ceo, J.J. Ferrada, W. H. Griest, J. M. Keller, and R. L. Schenley, Sampling and Analysis of Radioactive Liquid Wastes and Sludges in the Melton Valley and Evaporator Facility Storage Tanks at ORNL, ORNL/TM-11652, September 1990.

8. J.M. Keller, J. M. Giaquinto, and A. M. Meeks, Characterization of the MVST Waste Tanks Located at ORNL, ORNL/TM-13357, December 1996.

9. J. M. Keller, J. M. Giaquinto, and A. M. Meeks, Characterization of the BVEST Waste Tanks Located at ORNL, ORNL/TM-13358, January 1997.

10. Transuranic Waste Characterization Quality Assurance Program Plan, Rev. 0, CAO-94-1010, April 30, 1995

11. Radioactive Materials Analysis Laboratory - Oak Ridge National Laboratory (RMAL-ORNL) Quality Assurance Project Plan (QAPjP) for the Transuranic Waste Characterization Program (TWCP), Rev. 1, QAPX-CASD/RML-002, January 1996.

12. Radioactive Materials Analysis Laboratory Quality Assurance Plan for the Characterization of Radioactive Waste, QAP-X-CASD/RML-001, Rev. 1, June 1996

13. Waste Acceptance Criteria for the Waste Isolation Pilot Plant, WIPP-DOE-069, Rev. 5.0, April 1996.

14. Table B.1. Characteristics of Important Radionuclides, Integrated Database (IDB) Report, DOE/RW-0006, Rev. 11, September 1995.

15. M. R. Powell, Pacific Northwest Laboratory, Richland, Washington, personal communication to J. M. Giaquinto, March 26, 1997. 


\section{APPENDIX A}

Table A1 Instrument Detection Limits (IDL) for Analytical Measurements

\begin{tabular}{|c|c|c|}
\hline $\begin{array}{l}\text { Characteristic } \\
\text { (Analysis) }\end{array}$ & $\begin{array}{l}\text { Liquid } \\
\text { (mg/L) }\end{array}$ & $\begin{array}{c}\begin{array}{c}\text { Sludge } \\
\text { (mg/Kg) }\end{array} \\
\end{array}$ \\
\hline \multicolumn{3}{|c|}{ Physical properties and miscellaneous data } \\
\hline $\begin{array}{l}\text { TC } \\
\text { TIC } \\
\text { TOC }\end{array}$ & $\begin{array}{l}15 \\
15 \\
15\end{array}$ & - \\
\hline \multicolumn{3}{|l|}{ RCRA Metals } \\
\hline $\begin{array}{l}\mathrm{Ag} \\
\mathrm{As} \\
\mathrm{Ba} \\
\mathrm{Cd} \\
\mathrm{Cr} \\
\mathrm{Hg} \\
\mathrm{Ni} \\
\mathrm{Pb} \\
\mathrm{Se} \\
\mathrm{Tl}\end{array}$ & $\begin{array}{l}0.005 \\
0.005 \\
0.001 \\
0.006 \\
0.004 \\
0.0002 \\
0.009 \\
0.005 \\
0.005 \\
0.005\end{array}$ & $\begin{array}{l}0.5 \\
0.5 \\
0.1 \\
0.6 \\
0.4 \\
0.02 \\
0.9 \\
0.5 \\
0.5 \\
0.5\end{array}$ \\
\hline \multicolumn{3}{|l|}{ Process metals } \\
\hline $\begin{array}{l}\mathrm{Al} \\
\mathrm{B} \\
\mathrm{Be} \\
\mathrm{Ca} \\
\mathrm{Co} \\
\mathrm{C} \\
\mathrm{Cu} \\
\mathrm{Fe} \\
\mathrm{K} \\
\mathrm{Mg} \\
\mathrm{Mn} \\
\mathrm{Na} \\
\mathrm{P} \\
\mathrm{Sb} \\
\mathrm{Si} \\
\mathrm{Sr} \\
\mathrm{Th} \\
\mathrm{U} \\
\mathrm{V} \\
\mathrm{Zn} \\
\end{array}$ & $\begin{array}{l}0.02 \\
0.012 \\
0.0009 \\
0.01 \\
0.007 \\
0.005 \\
0.002 \\
0.003 \\
0.08 \\
0.02 \\
0.0009 \\
0.02 \\
0.02 \\
0.13 \\
0.013 \\
0.0003 \\
0.04 \\
0.07 \\
0.02 \\
0.02 \\
\end{array}$ & $\begin{array}{l}2.0 \\
1.2 \\
0.09 \\
1.0 \\
0.7 \\
0.5 \\
0.2 \\
0.3 \\
8.0 \\
2.0 \\
0.09 \\
2.0 \\
2.0 \\
13.0 \\
1.3 \\
0.03 \\
4.0 \\
7.0 \\
2.0 \\
2.0 \\
\end{array}$ \\
\hline \multicolumn{3}{|c|}{ Anions by ion chromatography } \\
\hline $\begin{array}{l}\text { Bromide } \\
\text { Chloride } \\
\text { Fluoride } \\
\text { Nitrate } \\
\text { Nitrite } \\
\text { Phosphate } \\
\text { Sulfate }\end{array}$ & $\begin{array}{l}0.05 \\
0.05 \\
0.05 \\
0.05 \\
0.01 \\
0.20 \\
0.10\end{array}$ & $\begin{array}{l}0.5 \\
0.5 \\
0.5 \\
0.5 \\
0.1 \\
2.0 \\
1.0\end{array}$ \\
\hline
\end{tabular}




\section{Radioactive Materials Analytical Laboratory}

Table A2 QC Acceptance Criteria for Radioactive Liquid/Solid Waste Samples

\begin{tabular}{|c|c|c|c|c|}
\hline Analysis & $\begin{array}{l}\text { Method (s) } \\
\text { CASD-AM- }\end{array}$ & $\begin{array}{l}\text { Quality Control } \\
\text { Check } \\
\text { (per batch) }\end{array}$ & $\begin{array}{c}\text { SW-846 } \\
\text { Acceptance } \\
\text { Criteria } \\
(\% \mathrm{D}, \% \mathbf{R}, \mathbf{R P D})^{\mathrm{C}}\end{array}$ & $\begin{array}{c}\text { RMAL } \\
\text { Acceptance } \\
\text { Criteria } \\
(\% D, \% R, \text { RPD })^{e}\end{array}$ \\
\hline $\begin{array}{l}\text { Metals by ICP-AES } \\
\text { (inductively coupled } \\
\text { plasminatomic } \\
\text { emission } \\
\text { spectroscopy) }\end{array}$ & SW846-6010A & $\begin{array}{l}\text { high standard } \\
\text { calibration verifications (ICV \& CCV) } \\
\text { calibration blark \& checks (ICB \& CCB)" } \\
\text { method blank (sample prep) } \\
\text { matrix spike } \\
\text { matrix spike duplicate or sample duplicate } \\
\text { laboratory control sample (sample prep)" } \\
\text { serial dilution (if interference suspected) } \\
\text { post digestion spiked }\end{array}$ & $\begin{array}{l} \pm 5 \% \mathrm{D} \\
\pm 10 \% \mathrm{D} \\
3 \times \mathrm{IDL} \\
3 \times \mathrm{IDL} \\
\pm 20 \% \mathrm{D} \\
\pm 20 \mathrm{RPD} \\
\text { none specified } \\
\pm 10 \% \mathrm{R} \\
\pm 20 \% \mathrm{D}\end{array}$ & $\begin{array}{c} \pm 5 \% \mathrm{D} \\
\pm 10 \% \mathrm{D} \\
3 \times \mathrm{IDL} \\
3 \times \mathrm{WLL} \\
\pm 25 \% \mathrm{D} \text { (liq.), } \pm 30 \% \mathrm{DD} \text { (solid) } \\
\pm 20 \mathrm{RPD} \text { (liq.), } \pm 30 \mathrm{RPD} \text { (solid) } \\
\pm 20 \% \mathrm{D} \\
\pm 10 \% \mathrm{R} \\
\pm 25 \% \mathrm{D} \text { (liq.), } \pm 30 \% \mathrm{D} \text { (solid) }\end{array}$ \\
\hline $\begin{array}{l}\text { Metals by ICP-MS } \\
\text { (inductively coupled } \\
\text { plasma-mass } \\
\text { spectrometry, } \\
\text { fully quantitative } \\
\text { method) }\end{array}$ & SW846-6020 & $\begin{array}{l}\text { calibration verifications (ICV \& CCV)" } \\
\text { calibration blank \& blank checks (CCB) } \\
\text { method blank (sample prep) } \\
\text { matrix spike } \\
\text { matrix spike duplicate or sample duplicate } \\
\text { laboratory control sample (sample prep) } \\
\text { internal standard } \\
\text { post digestion spiked }\end{array}$ & $\begin{array}{l} \pm 10 \% \mathrm{D} \\
3 \times \mathrm{IDL} \\
\text { none specified } \\
\text { none specified } \\
\pm 20 \mathrm{RPD} \\
\text { none specified } \\
30-120 \% \mathrm{R} \\
\pm 10 \% \mathrm{D}\end{array}$ & $\begin{array}{c} \pm 10 \% \mathrm{D} \\
3 \times \mathrm{IDL} \\
<10 \times \mathrm{IDL} \\
\pm 25 \% \mathrm{D} \text { (liq.), } \pm 30 \% \mathrm{D} \text { (solid) } \\
\pm 20 \mathrm{RPD} \text { (liq.), } \pm 30 \mathrm{RPD} \text { (solid) } \\
\pm 20 \% \mathrm{D} \\
\pm 30 \% \mathrm{D} \\
\pm 20 \% \mathrm{D}\end{array}$ \\
\hline $\begin{array}{l}\text { Metals by GFAA } \\
\text { (graphite furnace } \\
\text { atomic absorption) }\end{array}$ & SW846-7000A & $\begin{array}{l}\text { high standard } \\
\text { calibration verifieations (ICV \& CCV)' } \\
\text { method blank (sample prep) } \\
\text { matrix spike } \\
\text { matrix spike duplicate } \\
\text { laboratory control sample (sample prep)' } \\
\text { serial dilution (if interierence suspected) } \\
\text { post digestion spiked }\end{array}$ & $\begin{array}{l}\text { not required } \\
\pm 10 \% \mathrm{D}(\mathrm{ICV}), \pm 20 \% \mathrm{D}(\mathrm{CCV}) \\
\text { none specified } \\
\text { none specified } \\
\text { none specified } \\
\text { none specified } \\
\pm 10 \% \mathrm{R} \\
\pm 15 \% \mathrm{D}\end{array}$ & $\begin{array}{c} \pm 5 \% \mathrm{D} \\
\pm 10 \% \mathrm{D} \text { (ICV), } \pm 20 \% \mathrm{D}(\mathrm{CCV}) \\
<\times \mathrm{IDL} \\
\pm 25 \% \mathrm{D} \text { (liq.), } \pm 30 \% \mathrm{D} \text { (solid) } \\
\pm 20 \mathrm{RPD} \text { (liq.), } \pm 30 \mathrm{RPD} \text { (solid) } \\
\pm 25 \% \mathrm{D} \\
\pm 10 \% \mathrm{R} \\
\pm 25 \% \mathrm{D} \text { (tiq.), } \pm 30 \% \mathrm{D} \text { (solid) }\end{array}$ \\
\hline $\begin{array}{l}\text { Mercury by CVAA } \\
\text { (cold vapor atomic } \\
\text { absorption) }\end{array}$ & $\begin{array}{l}\text { SW846-7471A } \\
\text { SW846-7470 }\end{array}$ & $\begin{array}{l}\text { instrument blank } \\
\text { calibration verification (ICV \& CCV' } \\
\text { method blank (sample prep)' } \\
\text { laboratory control sample (sample prep) } \\
\text { matrix spike } \\
\text { matrix spike duplicate or sample duplicate } \\
\text { post digestion spiked }\end{array}$ & $\begin{array}{l}\text { none specified } \\
\text { none specified } \\
\text { none specified } \\
\text { none specified } \\
\text { none specified } \\
\text { none specified } \\
\text { none specified }\end{array}$ & $\begin{array}{c}<5 \times \mathrm{IDL} \\
\pm 10 \% \mathrm{D} \\
<\times \mathrm{IDL} \\
\pm 25 \% \mathrm{D} \\
\pm 25 \% \mathrm{D} \text { (iq.), } \pm 30 \% \mathrm{D} \text { (solid) } \\
\pm 20 \mathrm{RPD} \text { (iiq.), } \pm 30 \mathrm{RPD} \text { (solid) } \\
\pm 25 \% \mathrm{D} \text { (jiq), } \pm 30 \% \mathrm{D} \text { (solid) }\end{array}$ \\
\hline $\begin{array}{l}\text { Carbon (total } \\
\text { organic carbon, total } \\
\text { carbon, total } \\
\text { inorganic carbon) }\end{array}$ & SW846-9060 & $\begin{array}{l}\text { instrument blank } \\
\text { calibration verification (ICV \& CCV)' } \\
\text { matrix spike } \\
\text { matrix spike duplicate }\end{array}$ & $\begin{array}{l}\text { none specified } \\
\text { none specified } \\
\text { none specified } \\
\text { none specified }\end{array}$ & $\begin{array}{c}3 \times \mathrm{IDL} \\
\pm 10 \% \mathrm{D}(\mathrm{ICV}), \pm 20 \% \mathrm{D}(\mathrm{CCV}) \\
=25 \% \mathrm{D} \text { (liq.), } \pm 30 \% \mathrm{D} \text { (solid) } \\
\pm 20 \mathrm{RPD} \text { (liq.), } \pm 30 \mathrm{RPD} \text { (solid) }\end{array}$ \\
\hline $\begin{array}{l}\text { Arions by Ion } \\
\text { Chromatography } \\
\text { (IC) }\end{array}$ & SW846-9056 & $\begin{array}{l}\text { calibration verification (ICV \& CCV) } \\
\text { matrix spike } \\
\text { sample duplicate }\end{array}$ & $\begin{array}{c} \pm 10 \% \mathrm{D} \text { (ICV), } \pm 5 \% \mathrm{D} \text { (CCV) } \\
\text { none specified } \\
\text { none specified }\end{array}$ & $\begin{array}{c} \pm 10 \% \mathrm{D}(\mathrm{ICV}), \pm 15 \% \mathrm{D}(\mathrm{CCV}) \\
\pm 25 \% \mathrm{D} \\
\pm 20 \mathrm{RPD}\end{array}$ \\
\hline pH measurement & $\begin{array}{l}\text { SW846-9040A } \\
\text { SW846-9045B }\end{array}$ & $\begin{array}{l}\text { check standard } \\
\text { sample duplicate }\end{array}$ & $\begin{array}{l}\text { none specified } \\
\text { none specified }\end{array}$ & $\begin{array}{l} \pm 10 \% \mathrm{D} \\
\pm 20 \% \mathrm{D}\end{array}$ \\
\hline $\begin{array}{l}\text { Total and dissolved } \\
\text { solids (IS \& TDS) }\end{array}$ & $\begin{array}{l}\text { EPA600-160.2 } \\
\text { EPA600-160.3 }\end{array}$ & $\begin{array}{l}\text { sample duplicate } \\
\text { check standard }\end{array}$ & $\begin{array}{l}\text { none specified } \\
\text { none specified }\end{array}$ & $\begin{array}{c} \pm 10 \mathrm{mg} / 10 \mathrm{~mL} \text { sample } \\
=10 \% \mathrm{D}\end{array}$ \\
\hline $\begin{array}{l}\text { Carbonate and } \\
\text { bicarbonate titration }\end{array}$ & AC-MM-1 003105 & $\begin{array}{l}\text { sample duplicate } \\
\text { check standard }\end{array}$ & $\begin{array}{l}\text { none specified } \\
\text { none specified }\end{array}$ & $\begin{array}{l} \pm 20 \mathrm{RPD} \\
\pm 20 \% \mathrm{D}\end{array}$ \\
\hline Gross alpha/beta & $\begin{array}{l}\text { EPA-900.0 } \\
\text { RML-RA02 } \\
\text { RMLRA12 }\end{array}$ & $\begin{array}{l}\text { background check } \\
\text { calibration verification } \\
\text { method blank (optional) } \\
\text { sample duplicate } \\
\text { matrix spike }\end{array}$ & $\begin{array}{l}\text { none specified } \\
\text { none specified } \\
\text { none specified } \\
\text { none specified } \\
\text { none specified }\end{array}$ & $\begin{array}{l}\text { <3sigma daily change } \\
\quad \pm 10 \% \mathrm{D} \\
\text { evaluated for contamination } \\
\pm 25 \text { RPD (liq.), } \pm 30 \text { RPD (solid) } \\
\pm 25 \% \mathrm{D} \text { (liq.) \& } \pm 30 \% \mathrm{D} \text { (solid) }\end{array}$ \\
\hline $\begin{array}{l}\text { Nuclides by gamma } \\
\text { spectrometry }\end{array}$ & EPA-901.1 & $\begin{array}{l}\text { background check } \\
\text { calibration verification } \\
\text { sample duplicate }\end{array}$ & $\begin{array}{l}\text { none specified } \\
\text { none specified } \\
\text { none specified }\end{array}$ & $\begin{array}{c}<3 \text { sigma daily change } \\
\pm 10 \% \mathrm{D} \\
\pm 25 \% \mathrm{D} \text { (liq.) \& } 230 \% \mathrm{D} \text { (solio) }\end{array}$ \\
\hline Sr-90 determination & $\begin{array}{l}\text { RML-RA13 } \\
\text { EPA-905.0 }\end{array}$ & $\begin{array}{l}\text { method blank (optional) } \\
\text { laboratory control sample } \\
\text { matrix spike } \\
\text { matrix spike duplicate or sample duplicate } \\
\text { associated instrument QC }\end{array}$ & $\begin{array}{l}\text { none specified } \\
\text { none specified } \\
\text { none specified } \\
\text { none specified } \\
\text { none specified }\end{array}$ & $\begin{array}{l}\text { evaluated for contamination } \\
\qquad 20 \% \mathrm{D} \\
\pm 25 \% \mathrm{D} \text { (liq.) \& } \pm 30 \% \mathrm{DD} \text { (solid) } \\
\pm 25 \mathrm{RPD} \text { (liq.), } \pm 30 \mathrm{RPD} \text { (solid) } \\
\text { see gross alpha/beta criteria }\end{array}$ \\
\hline
\end{tabular}




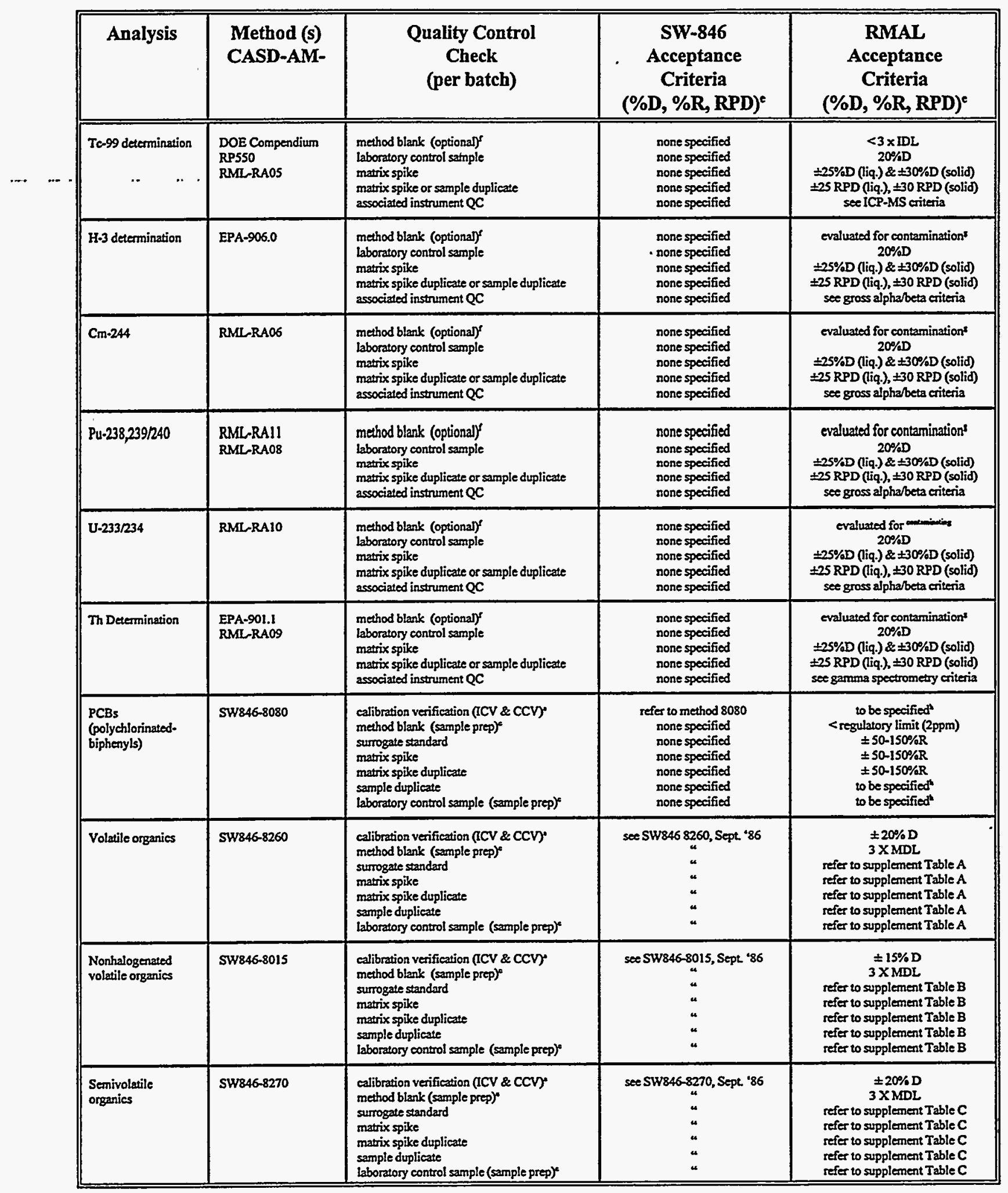

a Initial calibration verification (ICV) is typically performed at the beginning of a run to check the calibration and must be independent of the calibration standards. The continuing calibration verification (CCV) must also be independent of the calibration standards, but may be the same standard as the ICV. The CCV is typically analyzed every 10 samples and at the end of the run for metals analysis or every 12 samples for organic analysis. 
b The calibration blank is an instrument blank used in the calibration to initially determine the blank value and therefore used as blank subtraction. The continuing calibration blank (CCB) is also an instrument blank which is analyzed every 10 samples and at the end of the run, but is not used in blank subtraction, but only to monitor instrument contamination.

c Method blanks and laboratory control samples are only required if a sample preparation is performed before analysis. Sample preparation does not include dilutions or transfers to containers.

d Post digestion spikes are not necessary if the pre-digestion spike is in control. If this control does not meet the QC acceptance criteria, the post digestion spike should be performed.

e Acceptance criteria:

$\% \mathrm{D}=\%$ deviation from true value

$\% \mathrm{R}=\%$ recovery of true value

$\mathrm{RPD}=$ relative percent difference between two compared values

f Method blanks for radiochemical analysis are used to monitor cross contamination. However, due to the levels of radioactivity present in samples at the RMAL, the effect of contamination may be insignificant in most cases. Therefore, the requirement to analyze a method blank for radiochemical analysis is optional (i.e. at the discretion of the chemist or supervisor).

g Acceptance criteria for the method blanks performed for radiochemical analysis varies based upon the level of activity in the samples and the amount of background activity. A qualified chemist reviews the data from method blanks to determine if significant contamination is present.

h The acceptance criteria for PCB analyses which are not identified in this table, shall be specified at a later date. Currently, the Analytical Methods Group group leader specifies the QC criteria if different from SW846 and if not specified by the sample generator. 
SUPPLEMENT TABLE A2.1

Volatile Organic Analyses QC Limits

\begin{tabular}{|c|c|c|c|c|c|c|}
\hline$\overline{\overline{C A S} \#}$ & Compound & $\begin{array}{l}\text { Precision } \\
\text { (RPD) }\end{array}$ & $\begin{array}{c}\text { Accuracy } \\
(\% \mathrm{R})\end{array}$ & $\begin{array}{c}\mathrm{MDL} \\
(\mathrm{mg} / \mathrm{Kg})\end{array}$ & $\begin{array}{c}\text { PRQL } \\
\text { (mg/Kg) }\end{array}$ & $\begin{array}{c}\text { LCS } \\
(\% \mathrm{R}) \\
\end{array}$ \\
\hline $75-01-4$ & Vinyl Chloride & 5200 & $\mathrm{D}-251$ & 1 & 4 & $34-100$ \\
\hline $75-69-4$ & Trichlorofluoromethane & $\leq 110$ & $17-181$ & 1 & 10 & $47-103$ \\
\hline $76-13-1$ & 1,1,2-Trichloro-1,2-2-Trifluoroethane & $\leq 50$ & $60-150$ & 1 & 10 & $49-105$ \\
\hline $75-35-4$ & 1,1-Dichloroethylene & $\leq 250$ & $\mathrm{D}-234$ & 1 & 10 & $43-100$ \\
\hline $75-9-2$ & Methylene Chloride & $\leq 50$ & $\mathrm{D}-221$ & 1 & 10 & $67-108$ \\
\hline $75-15-0$ & Carbon Disulfide & $\leq 50$ & $60-150$ & 1 & 10 & $36-100$ \\
\hline $67-66-3$ & Chloroform & $\leq 44$ & $51-138$ & 1 & 10 & $72-111$ \\
\hline $107-6-2$ & 1,2-Dichloroethane & $\leq 42$ & $49-155$ & 1 & 10 & $76-112$ \\
\hline $71-55-6$ & 1,1,1-Trichloroethane & $\leq 33$ & $52-162$ & 1 & 10 & $71-110$ \\
\hline $56-23-5$ & Carbon Tetrachloride & $\leq 30$ & $70-140$ & 1 & 10 & $54-115$ \\
\hline $71-43-2$ & Benzene & $\leq 45$ & $37-151$ & 1 & 10 & $70-109$ \\
\hline $79-1-6$ & Trichloroethylene & $\leq 36$ & $71-157$ & 1 & 10 & $80-120$ \\
\hline $79-0-5$ & 1,1,2-Trichloroethane & $\leq 38$ & $52-150$ & 1 & 10 & $80-120$ \\
\hline $75-25-2$ & Bromoform & $\leq 47$ & $45-169$ & 1 & 10 & $61-115$ \\
\hline $108-88-3$ & Toluene & $\leq 29$ & $47-150$ & 1 & 10 & $80-120$ \\
\hline $127-18-4$ & Tetrachloroethylene & $\leq 29$ & $64-148$ & 1 & 10 & $80-120$ \\
\hline $108-90-7$ & Chlorobenzene & 538 & $37-160$ & 1 & 10 & $80-120$ \\
\hline $100-41-4$ & Ethylbenzene & $\leq 43$ & $37-162$ & 1 & 10 & $80-120$ \\
\hline $1330-20-7$ & Xylenes & 550 & $60-150$ & 1 & 10 & $80-120$ \\
\hline $79-34-5$ & 1,1,2,2-Tetrachloroethane & $\leq 55$ & $46-157$ & 1 & 10 & $67-117$ \\
\hline $106-46-7$ & 1,4-Dichlorobenzene & $\leq 60$ & $18-190$ & 1 & 10 & $80-120$ \\
\hline $95-50-1$ & ortho-Dichlorobenzene & $\leq 60$ & $18-190$ & 1 & 10 & $80-112$ \\
\hline $60-29-7$ & Ethyl Ether & $\leq 50$ & $60-150$ & 1. & 10 & $54-100$ \\
\hline \multicolumn{7}{|l|}{ Sprrogates } \\
\hline & 1,2-Dichloroethane-d & & $61-129$ & & & \\
\hline & Toluene- $d_{3}$ & & $89-118$ & & & \\
\hline & 4-Bromofluorobenzene & & $93-107$ & & & \\
\hline
\end{tabular}


SUPPLEMENT TABLE A2.2

\section{Nonhalogenated Volatile Organic Analyses QC Limits}

\begin{tabular}{|c|c|c|c|c|c|c|}
\hline CAS\# & Compound & $\begin{array}{c}\begin{array}{c}\text { Precision } \\
\text { (RPD) }\end{array} \\
\end{array}$ & $\begin{array}{c}\text { Accuracy } \\
(\% \mathrm{R}) \\
\end{array}$ & $\begin{array}{c}\text { MDL } \\
(\mathrm{mg} / \mathrm{Kg}) \\
\end{array}$ & $\begin{array}{c}\text { PRQL } \\
(\mathrm{mg} / \mathrm{Kg}) \\
\end{array}$ & $\begin{array}{r}\text { LCS } \\
(\% \mathrm{R}) \\
\end{array}$ \\
\hline $67-56-1$ & Methanol & $\leq 50$ & $60-150$ & 10 & 100 & $.49-145$ \\
\hline $67-64-1$ & Acetone & $\leq 50$ & $60-150$ & 10 & 100 & 61-136 \\
\hline $78-93-3$ & $\begin{array}{l}\text { Methyl Ethyl } \\
\text { Ketone }\end{array}$ & $\leq 50$ & $60-150$ & 10 & 100 & $62-134$ \\
\hline $78-83-1$ & Isobutanol & $\leq 50$ & $60-150$ & 10 & 100 & $52-126$ \\
\hline $71-36-3$ & Butanol & $\leq 50$ & $60-150$ & 10 & 100 & $50-110$ \\
\hline $110-86-1$ & Pyridine & $\leq 50$ & $60-150$ & 10 & 100 & $64-122$ \\
\hline \multicolumn{7}{|l|}{ Syrogate } \\
\hline $71-23-8$ & n-Propanol & & $60-150$ & & & \\
\hline
\end{tabular}

SUPPLEMENT TABLE A2.3

Semivolatile Organic Analyses QC Limits

\begin{tabular}{|c|c|c|c|c|c|c|}
\hline$\overline{\mathrm{CAS} \#}$ & Compound & $\begin{array}{c}\text { Precision } \\
\text { (RPD) } \\
\end{array}$ & $\begin{array}{c}\text { Accuracy } \\
(\% \text { R) } \\
\end{array}$ & $\begin{array}{c}\mathrm{MDL} \\
(\mathrm{mg} / \mathrm{Kg}) \\
\end{array}$ & $\begin{array}{c}\text { PRQL } \\
\text { (mg/Kg) }\end{array}$ & $\begin{array}{c}\mathrm{LCS} \\
(\% \mathrm{R}) \\
\end{array}$ \\
\hline $95-48-7$ & 2-Methylphenol & $\leq 50$ & $60-150$ & 5 & 40 & $46-104$ \\
\hline $67-72-1$ & Hexachloroethane & $\leq 44$ & $40-113$ & 5 & 40 & $38-100$ \\
\hline $106-44-5$ & 4-Methylphenol & $\leq 50$ & $60-150$ & 5 & 40 & $46-114$ \\
\hline $98-95-3$ & Nitrobenzene & $\leq 72$ & $35-180$ & 5 & 40 & $46-100$ \\
\hline $121-14-2$ & 2,4-Dinitrotoluene & $\leq 46$ & $39-139$ & 0.3 & 2.6 & $54-146$ \\
\hline $118-74-1$ & Hexachlorobenzene & $\leq 319$ & D-152 & 0.3 & 2.6 & $52-115$ \\
\hline $87-86-5$ & Pentachlorophenol & $\leq 128$ & $14-176$ & 5 & 40 & $54-130$ \\
\hline $51-28-5$ & 2,4-Dinitrophenol & $\leq 119$ & D-172 & 5 & 40 & $47-100$ \\
\hline \multicolumn{7}{|l|}{ Surrogates } \\
\hline \multirow[t]{3}{*}{$367-12-4$} & 2-Fluorophenol & & D-107 & & & . \\
\hline & Phenol-d $d_{s}$ & & $8-142$ & & & \\
\hline & Nitrobenzene-d $d_{s}$ & & $28-117$ & & & \\
\hline \multirow[t]{3}{*}{$321-60-8$} & 2-Fluorobiphenyl & & $24-144$ & & & \\
\hline & 2,4,6-Tribromophenol & & D-100 & & & \\
\hline & Terphenyl- $d_{u}$ & & $\mathrm{D}-226$ & & & \\
\hline
\end{tabular}




\section{APPENDIX B}

This section includes three tables of information and measurements that may be of value to the data users. The first Table B1, includes the field measurements taken from the top of the tank to each phase change (air/liquid, liquid/sludge, and bottom of the tank). Table B1 also includes the total mass and/or activity for some of the major species in the sludge of general interest to the data users.

The dose measurement taken in during the field sampling for the liquid and sludge samples are included in Table B2 and Table B3. The dose measurements were taken at contact with the sampling container ( $250 \mathrm{~mL}$ I-Chem jar) for the liquids and at contact with the one inch core sludge sampling device. 
Table B1 Total Mass and Activity for Selected Species in the OHF Sludge

\begin{tabular}{|c|c|c|c|c|c|c|c|}
\hline$\overline{\text { Measurement }}$ & & $\overline{T 1}$ & 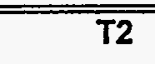 & 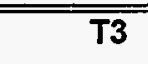 & $\overline{T 4}$ & T9 & \\
\hline Depth to top of liquid & (in.) & 89.5 & 88.0 & 151.0 & 106.0 & 119.0 & \\
\hline Depth to top of sludge & (in.) & 152.0 & 149.0 & 160.0 & 166.5 & 163.0 & \\
\hline Depth to bottom of tank & (in.) & 156.0 & 156.0 & 181.0 & 178.0 & 174.0 & \\
\hline Depth of supernatant & (in.) & 62.5 & 61.0 & 9.0 & 60.5 & 44.0 & \\
\hline Depth of sludge & (in.) & 4.0 & 7.0 & 21.0 & 11.5 & 11.0 & \\
\hline \multicolumn{4}{|c|}{ 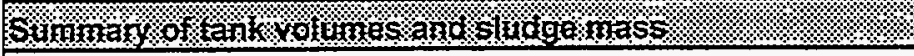 } & s. & 㽷 & & rolotat \\
\hline Volume of Supernatant & (L) & 40810 & 40240 & 7420 & 55990 & 18660 & 163120 \\
\hline Volume of Sludge (max.) & (L) & 5340 & 5910 & 11810 & 8740 & 4320 & 36120 \\
\hline Density of Sludge & $(\mathrm{Kg} / \mathrm{L})$ & 1.33 & 1.33 & 1.31 & 1.21 & 1.16 & \\
\hline Mass of Sludge & $(\mathrm{Kg})$ & 7102.2 & 7860.3 & 15471.1 & 10575.4 & 5011.2 & 46020.2 \\
\hline$\%$ of Total sludge & $(\%)$ & $15.4 \%$ & $17.1 \%$ & $33.6 \%$ & $23.0 \%$ & $10.9 \%$ & \\
\hline \multicolumn{6}{|c|}{ 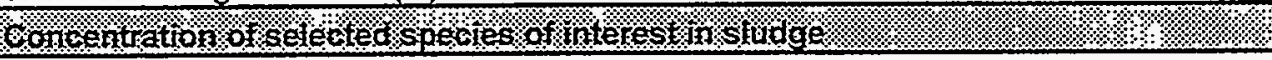 } & $\sqrt[4]{4.18}$ & \\
\hline Thorium & $(\mathrm{mg} / \mathrm{Kg})$ & 90500 & 94300 & 77500 & 124000 & 56800 & \\
\hline Uranium & $(\mathrm{mg} / \mathrm{Kg})$ & 2420 & 2090 & 5920 & 7870 & 2510 & \\
\hline Plutonium & $(\mathrm{mg} / \mathrm{Kg})$ & 3.2 & 3.4 & 2.1 & 4.0 & 3.1 & \\
\hline $233 \mathrm{U}$ & $(\mathrm{mg} / \mathrm{Kg})$ & 22.0 & 21.9 & 42.9 & 68.6 & 17.9 & \\
\hline $235 \mathrm{U}$ & $(\mathrm{mg} / \mathrm{Kg})$ & 10.3 & 7.2 & 23.4 & 29.5 & 12.4 & \\
\hline${ }^{239} \mathrm{Pu}$ & $(\mathrm{mg} / \mathrm{Kg})$ & 2.4 & 2.4 & 1.4 & 2.8 & 2.4 & \\
\hline \multicolumn{4}{|c|}{ 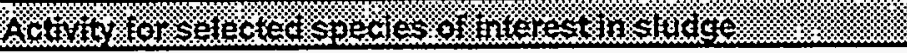 } & 8 & & 18.1 .1 .1$. & \\
\hline${ }^{90} \mathrm{Sr}$ & $(\mathrm{Bq} / \mathrm{g})$ & $2.0 E \div 07$ & $1.8 E+07$ & $8.5 E+06$ & $1.6 E+07$ & $2.0 \mathrm{E}+07$ & \\
\hline${ }^{137} \mathrm{Cs}$ & $(\mathrm{Bq} / \mathrm{g})$ & $3.9 E+05$ & $3.5 \mathrm{E}+05$ & $1.6 E+06$ & $3.4 E+05$ & $2.6 \mathrm{E}+05$ & \\
\hline $233 \mathrm{U}$ & $(B q / g)$ & $7.9 E+03$ & $7.8 \mathrm{E}+03$ & $1.5 E+04$ & $2.4 E+04$ & $6.4 \mathrm{E}+03$ & \\
\hline${ }^{238} \mathrm{Pu}$ & $(\mathrm{Bq} / \mathrm{g})$ & $2.9 E+04$ & $2.3 E+04$ & $1.1 E+04$ & $2.2 E+04$ & $4.8 E+04$ & \\
\hline${ }^{241} \mathrm{Am}$ & $(B q / g)$ & $5.2 \mathrm{E}+04$ & 2.6E+04 & $1.5 E+04$ & $8.0 E+03$ & $1.3 E+04$ & \\
\hline${ }^{244} \mathrm{Cm}$ & $(B q / g)$ & $3.5 E+05$ & $4.6 \mathrm{E}+05$ & $2.5 E+05$ & $5.3 E+05$ & $2.7 E+05$ & \\
\hline \multicolumn{5}{|c|}{ 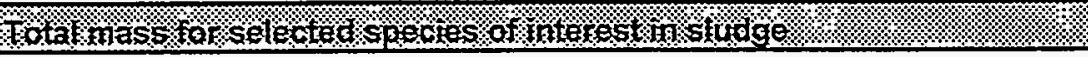 } & & & Kotat \\
\hline Thorium & $(\mathrm{Kg})$ & 642.7 & 741.2 & 1199.0 & 1311.3 & 284.6 & 4179.0 \\
\hline Uranium & $(\mathrm{Kg})$ & 17.2 & 16.4 & 91.6 & 83.2 & 12.6 & 221.0 \\
\hline Plutonium & $(\mathrm{Kg})$ & 0.023 & 0.027 & 0.032 & 0.043 & 0.016 & 0.140 \\
\hline${ }^{233} \mathrm{U}$ & $(\mathrm{Kg})$ & 0.156 & 0.172 & 0.664 & 0.725 & 0.090 & 1.807 \\
\hline $235 \mathrm{U}$ & $(\mathrm{Kg})$ & 0.073 & 0.057 & 0.362 & 0.312 & 0.062 & 0.866 \\
\hline${ }^{239} \mathrm{Pu}$ & $(\mathrm{Kg})$ & 0.017 & 0.019 & 0.022 & 0.030 & 0.012 & 0.100 \\
\hline \multicolumn{5}{|c|}{ 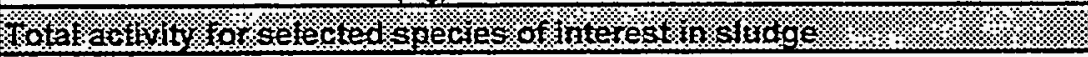 } & \multicolumn{2}{|c|}{ 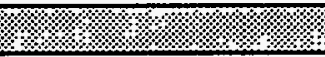 } & \%orata \\
\hline${ }^{90} \mathrm{Sr}$ & (Ci) & 3839.03 & 3823.93 & 3554.17 & 4573.15 & 2708.76 & 18499.0 \\
\hline${ }^{137} \mathrm{Cs}$ & (Ci) & 74.86 & 74.35 & 669.02 & 97.18 & 35.21 & 950.6 \\
\hline $233 \mathrm{U}$ & (Ci) & 1.52 & 1.66 & 6.27 & 6.86 & 0.87 & 17.2 \\
\hline${ }^{238} \mathrm{Pu}$ & (Ci) & 5.57 & 4.89 & 4.60 & 6.29 & 6.50 & 27.8 \\
\hline${ }^{241} \mathrm{Am}$ & (Ci) & 9.98 & 5.52 & 6.27 & 2.29 & 1.76 & 25.8 \\
\hline${ }^{244} \mathrm{Cm}$ & (Ci) & 67.18 & 97.72 & 104.53 & 151.49 & 36.57 & 457.5 \\
\hline
\end{tabular}




\section{Table B2 Field Dose Measurements on OHF Liquid Samples}

\begin{tabular}{lrrrrr}
\hline \hline & \multicolumn{3}{c}{$\begin{array}{c}\text { Supernatant } \\
\text { (mRad/hr) }\end{array}$} \\
\cline { 2 - 7 } Tank & L1 & L2 & L3 & L4 & $\begin{array}{c}\text { Date } \\
\text { sampled }\end{array}$ \\
\hline T1-North & 25 & 25 & 25 & 28 & $01 / 07 / 97$ \\
T1-Center & 25 & 27 & - & - & $01 / 23 / 96$ \\
T1-South & 25 & 25 & 50 & 27 & $01 / 07 / 97$ \\
T2-North & 40 & 42 & - & 50 & $01 / 07 / 97$ \\
T2-Center & 55 & 55 & 45 & - & $01 / 23 / 96$ \\
T2-South & 45 & 70 & 70 & 45 & $01 / 07 / 97$ \\
T3-North & 65 & 80 & 60 & 60 & $12 / 17 / 96$ \\
T3-Center & 75 & 60 & 60 & 65 & $01 / 17 / 96$ \\
T3-South & 60 & 60 & 70 & 60 & $01 / 17 / 96$ \\
T4-North & 60 & 80 & - & - & $01 / 17 / 97$ \\
T4-Center & 70 & 77 & 65 & 70 & $01 / 07 / 97$ \\
T4-South & 70 & 29 & 35 & 35 & $12 / 17 / 96$ \\
T9-North & 30 & 41 & - & - & $01 / 23 / 96$ \\
T9-Center & 40 & 33 & 33 & 30 & $12 / 17 / 96$ \\
T9-South & 35 & & & &
\end{tabular}

Table B3 Field Dose Measurements on OHF Sludge Samples

\begin{tabular}{|c|c|c|c|c|c|c|c|}
\hline \multirow[b]{2}{*}{ Tank } & \multicolumn{7}{|c|}{$\begin{array}{l}\text { Sludge } \\
\text { (mRad/hr) }\end{array}$} \\
\hline & S1 & s2 & $53^{2}$ & $54^{2}$ & $55^{a}$ & $56^{2}$ & $\begin{array}{c}\text { Date } \\
\text { sampled }\end{array}$ \\
\hline T1-North & 150 & 140 & - & - & - & - & $01 / 29 / 97$ \\
\hline T1-Center & 18000 & 20000 & - & - & - & - & $03 / 12 / 96$ \\
\hline T1-South & 1100 & 3700 & 3200 & 2800 & 6000 & 5000 & $01 / 29 / 97$ \\
\hline T2-North & 140 & 800 & - & - & - & - & 01/29/97 \\
\hline T2-Center & 35000 & 50000 & - & - & - & & $03 / 05 / 96$ \\
\hline T2-South & 5000 & 3000 & 6500 & 6500 & - & - & $01 / 29 / 97$ \\
\hline T3-North & 1100 & 800 & - & - & - & - & $01 / 27 / 97$ \\
\hline T3-Center & 15000 & - & - & - & - & - & $02 / 29 / 96$ \\
\hline T3-South & 1300 & 1500 & - & - & - & - & $01 / 27 / 97$ \\
\hline T4-North & 1000 & 1400 & - & - & - & - & 01/28/97 \\
\hline T4-Center & 30000 & - & - & - & - & - & $02 / 27 / 96$ \\
\hline T4-South & 3000 & - & - & - & - & - & $01 / 28 / 97$ \\
\hline T9-North & 4000 & 2000 & - & - & - & - & $01 / 27 / 97$ \\
\hline T9-Center & 50000 & - & - & - & - & - & $02 / 21 / 96$ \\
\hline T9-South & 2500 & 5000 & 7000 & 4500 & 4000 & 10000 & $01 / 27 / 97$ \\
\hline
\end{tabular}

Additional sludge samples were collected on $02 / 06 / 97$ for rheometry measurements.

Note: All dose measurements measured on contact with sampling device. 
Table B4 Laboratory Dose Measurements on OHF Sludge

\begin{tabular}{|ccccccc|}
\hline Tank & $\begin{array}{c}\text { Wet } \\
\text { Weight } \\
\text { (g) }\end{array}$ & $\begin{array}{c}\text { Dry } \\
\text { Weight } \\
(\mathbf{g})\end{array}$ & $\begin{array}{c}\text { Water } \\
\text { Content } \\
(\%)\end{array}$ & $\begin{array}{c}\text { Wet Beta } \\
\text { Dose } \\
(\text { Rad/h/g) }\end{array}$ & $\begin{array}{c}\text { Dry Beta } \\
\text { Dose } \\
\text { (Rad/h/g) }\end{array}$ & $\begin{array}{c}\text { Dry Gamma } \\
\text { Dose } \\
\text { (Rad/h/g) }\end{array}$ \\
\hline T1-North & 1.6436 & 0.3524 & 78.6 & 3.7 & 28.4 & 0.017 \\
T1-South & 1.1944 & 0.3253 & 72.8 & 7.5 & 46.1 & 0.025 \\
\hline T2-North & 1.3736 & 0.2911 & 78.8 & 4.4 & 30.9 & 0.024 \\
T2-South & 1.4939 & 0.3445 & 76.9 & 8.0 & 52.2 & 0.017 \\
\hline T3-North & 0.9262 & 0.2687 & 71.0 & 4.3 & 18.6 & 0.019 \\
T3-South & 1.2492 & 0.3596 & 71.2 & 4.0 & 22.2 & 0.017 \\
\hline T4-North & 0.8435 & 0.1691 & 80.0 & 7.1 & 59.1 & 0.053 \\
T4-South & 0.9790 & 0.1982 & 79.8 & 9.2 & 65.6 & 0.030 \\
\hline T9-North & 1.0693 & 0.2934 & 72.6 & 6.5 & 30.7 & 0.020 \\
T9-South & 1.0624 & 0.3735 & 64.8 & 7.5 & 34.8 & 0.021 \\
\hline
\end{tabular}

Notes: 1. All dose measurements done with Eberline RSO-20 at 1 in. from the surface of the sample, Open window $=$ beta+gamma, Closed window = gamma only.

2. Samples were spread out over a $43 \mathrm{~mm}$ weighing tin and dried at an average oven temperature of $110^{\circ} \mathrm{C}$. 


\section{APPENDIX C}

\section{Introduction to Rheological Models for Sludge Flow Behavior}

The mathematical models that describe the sludge flow behavior and the definition of some common terms used in Rheometry are discussed in this section. The cohesive behavior of a sludge in an undisturbed state can be considered to be a pseudo-solid. In order to mobilize and pump the sludge as a slurry some force must be applied to the undisturbed sludge to go from the pseudo-solid state to a viscous fluid or slurry state. A measure of this force required to transform the sludge into a fluid state is defined as the shear strength $\left(\tau_{s}\right)$.

The shear stress/shear rate or flow behavior for non-Newtonian fluids can be described by the power law model as follows,

$$
\tau=k \dot{\gamma}^{n}
$$

where, $\quad \begin{array}{ll}\mathrm{k}= & \text { consistency index }\left(\mathrm{Pa}-\mathrm{s}^{\mathrm{n}}\right), \\ \mathrm{n}= & \text { flow behavior index, } \\ \tau= & \text { shear stress }(\mathrm{Pa}), \text { and } \\ \gamma= & \text { shear rate }\left(\mathrm{s}^{-1}\right) .\end{array}$

If the flow behavior index, $\mathrm{n}=1$, the fluid is defined as Newtonian (ideal); if $\mathrm{n}<1$, the fluid is pseudoplastic; and if $\mathrm{n}>1$, the fluid shows dilatant behavior. The shear stress/shear rate behavior of these fluids are illustrated in Fig. C1. Many non-Newtonian fluids, such as a sludge slurry, require some minimum shear stress to flow. This shear stress needed to initiate flow is called the yield stress $\left(\tau_{y}\right)$, and fluids that display this behavior can be described by a modified form of the power law model:

$$
\tau=k \dot{\gamma}^{n}+\tau_{y}
$$

As illustrated in Fig. C2, the shear stress/shear rate flow curves for the modified power law behavior is very similar to the curves in Fig. $\mathrm{C} 1$, but are displaced from the origin by an amount equal to the yield stress. 
Figure C1 Example Rheograms for Non-Newtonian Fluids without Yield Stress

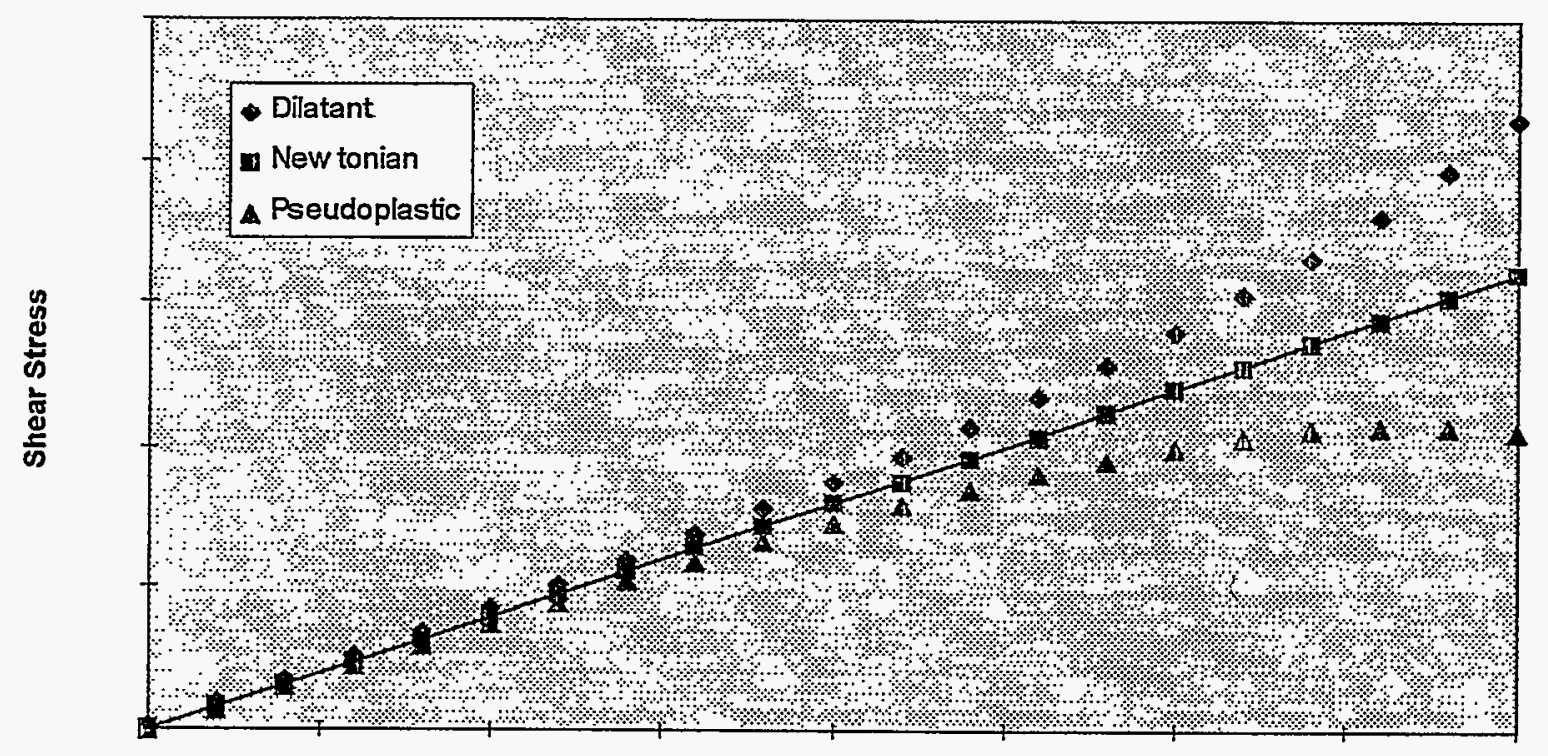

Shear Rate

Figure C2 Example of Rheograms for Non-Newtonian Fluids with Yield Stress

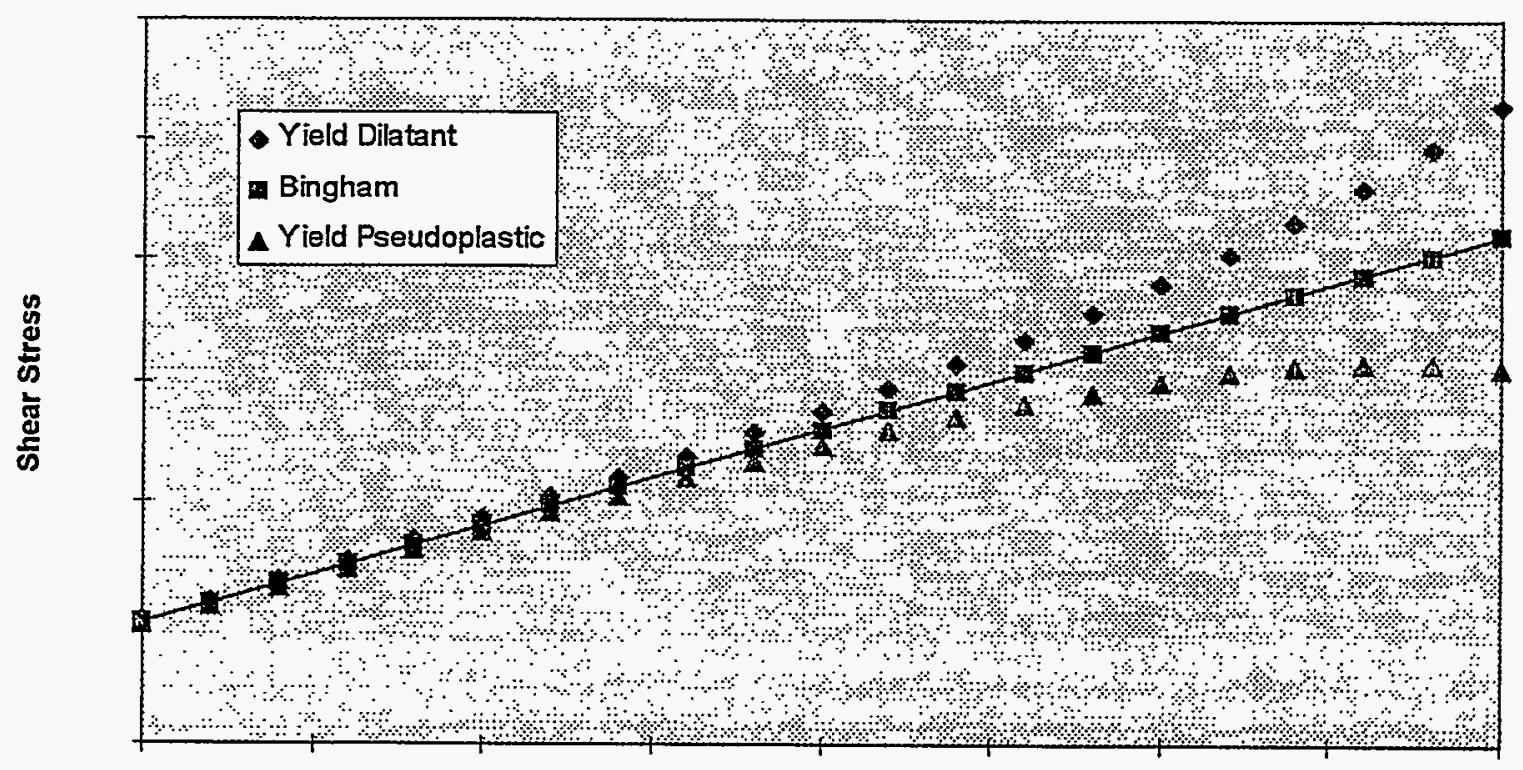

Shear Rate 
The pseudoplastic behavior shown in Fig. $\mathrm{C} 1$ falls below the Newtonian curve which indicates that at high shear rates a pseudoplastic fluid "thins out" such that less stress is required to maintain fluid motion. This pseudoplastic behavior is also called "shear-thinning". In contrast, the dilatant fluid exhibits "shear-thickening" behavior. Fluids that become thinner with time when stirred at a constant shear rate are called thixotropic, and fluids that thicken with time are called rheopectic. When dealing with sludge and slurry samples it is common practice to just note thixotropic behavior rather that to describe it in mathematical terms. Reporting the shear stress required to initiate motion in an undisturbed sludge, defined as the shear strength $\left(\tau_{s}\right)$, is usually sufficient for most applications.

In terms of sludge mobilization, an undisturbed sludge initially behaves as a pseudo-solid with a characteristic shear strength $\left(\tau_{s}\right)$. As the sludge is initially mixed the slurry becomes a thixotropic fluid when the shear strength is exceeded and as the mixing continues the fluid reaches a steady state condition where the slurry behaves as a power law fluid.

Viscosity is defined as the ratio of shear stress to shear rate,

$$
\eta=\frac{\tau}{\dot{\gamma}}
$$

In other words, viscosity is an indication of the stress required to cause a fluid to flow at a given rate. Fluids for which the viscosity is constant for all values of shear rate are defined as Newtonian fluids and as shown in Fig. $\mathrm{Cl}$ the shear stress/shear rate curve for Newtonian fluid is a straight line with a slope equal to the viscosity $(\eta)$ and a y-intercept of zero. In practice, most sludge or slurry based systems show non-Newtonian fluid behavior and the viscosity varies with the shear rate.

For additional background, a good review on the rheometry of radioactive sludge and slurry samples can be found in a 1987 report** from Pacific Northwest Laboratory (PNL).

\footnotetext{
** W. O. Heath, Development of an In-Situ Method to Define the Rheological properties of Slurries and Sludges Stored in Underground Tanks, PNL-6083 (April 1987).
} 
C-4 


\section{INTERNAL DISTRIBUTION}

1. J.F. Alexander

2. J. S. Baldwin

3. C. K. Bayne

4. E. C. Beahm

5. C. A. Bednarz

6. J.M. Begovich

7. D. A. Bostick

8-9. Central Research Library

10. J. A. Chapman

11. A. G. Croff

12. S. M. DePaoli

13. J.R. DeVore

14. B. Z. Egan

15-17. J. M. Giaquinto

18. T. M. Gilliam

19. W. H. Griest

20. T. D. Hylton

21. R. T. Jubin

22-24. J. M. Keller

25. C. M. Kendrick

26. T. E. Kent

27-28. Laboratory Records Department - RC

29. D. D. Lee

30. B. E. Lewis

31. A. J. Lucero

32. J. J. Maddox

33. R. C. Mason

34. A. J. Mattus

35. C. P. McGinnis

36. L. E. McNeese

37-39. A. M. Meeks

40. T. P. Mills

41. T. H. Monk

42. J. W. Moore

43. T. W. Morris

44. T. E. Myrick

45. C. D. Parks

46. J. H. Platfoot

47. M. L. Poutsma

48. S. M. Robinson

49. S. T. Rudell

50. T. F. Scanlan
51. F. J. Schultz

52. C. B. Scott

53. D. H. Smith

54. M. B. Sears

55. J. L. Snyder

56. R. D. Spence

57. R. C. Stewart

58. J. R. Stokely

59. P. A. Taylor

60. N. A. Teasley, Jr.

61. L. M. Toth

62. J.R. Trabalka

63. S. D. Van Hoesen

64. J. F. Walker, Jr.

65. J. S. Watson

66. T.D. Welch

67. J. H. Wilson

68. ER Document Management Center 